\title{
A review of the tribes of Deltocephalinae (Hemiptera: Auchenorrhyncha: Cicadellidae)
}

\author{
James N. ZAHNISER ${ }^{1, *} \&$ Chris H. DIETRICH ${ }^{2}$ \\ 1,2 Illinois Natural History Survey, Prairie Research Institute, \\ University of Illinois at Urbana-Champaign, \\ 1816 S. Oak St., Champaign, IL 61820, United States of America \\ * corresponding author e-mail: zahniser@illinois.edu \\ ${ }^{1}$ urn:lsid:zoobank.org:author:C44D6E44-FA1C-4B29-B7BB-FAF5940CD225 \\ ${ }^{2}$ urn:lsid:zoobank.org:author:82FCB86C-54B4-456A-AE5E-D7847D271CB9
}

\begin{abstract}
The classification of the largest subfamily of leafhoppers, Deltocephalinae, including 38 tribes, 923 genera, and 6683 valid species, is reviewed and revised. An updated phylogeny of the subfamily based on molecular (28S, Histone H3) and morphological data and an expanded taxon sample (37 taxa not included in previous analyses) is presented. Based on the results of these analyses and on the morphological examination of many representatives of the subfamily, the classification of the tribes and subtribes of Deltocephalinae is revised. Complete morphological descriptions, illustrations, lists of the included genera, and notes on their distribution, ecology, and important vector species are provided for the 38 recognized tribes and 18 subtribes. A dichotomous key to the tribes is provided. All names in the taxonomic treatments are hyperlinked to online resources for individual taxa which are supported by a comprehensive database for Deltocephalinae compiled using the taxonomic database software package 3I. The online functionality includes an interactive key to tribes and subtribes and advanced database searching options. Each taxon (subspecies through subfamily) has a unique taxon webpage providing nomenclatural information, lists of included taxa, an automated description (if available), images (if available), distributional information, bibliographic references and links to outside resources. Some observations and trends regarding the history of taxonomic descriptions in Deltocephalinae are reported. Four new tribes are described: Bahitini tribe nov. (25 genera), Bonsapeiini tribe nov. (21 genera), Phlepsiini tribe nov. (4 genera), and Vartini tribe nov. (7 genera). The circumscription and morphological characterization of Scaphoideini Oman, 1943 (61 genera) is substantially revised. Eleven new species are described: Acostemma stilleri sp. nov., Arrugada linnavuorii sp. nov., Drabescus zhangi sp. nov., Parabolopona webbi sp. nov., Goniagnathus emeljanovi sp. nov., Hecalus hamiltoni sp. nov., Scaphoideus omani sp. nov., Dwightla delongi sp. nov., Abimwa knighti sp. nov., Gannia viraktamathi sp. nov., and Doratulina dmitrievi sp. nov. Some family-group level taxonomic changes are made: Platymetopiini Haupt, 1929, Anoterostemmini Haupt, 1929, and Allygidiina Dmitriev, 2006 are synonymized with Athysanini Van Duzee, 1892, syn. nov.; Procepitini Dmitriev, 2002 is synonymized with Cicadulini Van Duzee, 1892, syn. nov.; Listrophorini Boulard, 1971 is synonymized with Chiasmini Distant, 1908, syn. nov.; Adamini Linnavuori \& Al-Ne'amy, 1983, Dwightlini McKamey, 2003, and Ianeirini Linnavuori, 1978 are synonymized with Selenocephalini Fieber, 1872 syn.nov., and all three are now recognized as valid subtribes in their parent tribe. New placements of many genera to tribe and subtribe are made, and these are described in individual taxon treatments.
\end{abstract}


Keywords. Leafhopper, classification, taxonomy, phylogeny, database, interactive key, cybertaxonomy.

Zahniser J.N. \& Dietrich C.H. 2013. A review of the tribes of Deltocephalinae (Hemiptera: Auchenorrhyncha: Cicadellidae). European Journal of Taxonomy 45: 1-211. http://dx.doi.org/10.5852/ejt.2013.45

\section{Introduction}

Deltocephalinae contains 6683 valid species and 923 genera (Fig. 1) currently making it the largest subfamily of Cicadellidae based on the number of described species. The subfamily is distributed worldwide, and it contains the majority of leafhopper vectors of economically important plant diseases, some of which cause significant damage and economic loss. Many species feed on herbaceous or woody dicotyledonous plants, while about $1 / 3$ of the tribes specialize on grass and sedge hosts and are particularly diverse and abundant in grassland ecosystems.

The family-group classification of Deltocephalinae has been somewhat controversial and has seen many changes over the past 50 years. The controversy has stemmed in part from the lack of global revisions and phylogenetic studies that provide the scope needed to address the classification of this cosmopolitan group of leafhoppers. Also, taxonomic concepts of many family-group taxa have often not been clearly stated or universally agreed upon, or even if they have, their circumscriptions have often not been tested by examination of multiple character sets or in phylogenetic analyses. These deficiencies have been partly addressed by taxonomic revisions (e.g., Linnavuori \& Al-Ne'amy 1983, Knight \& Webb 1993, Zhang \& Webb 1996, Dietrich \& Rakitov 2002, Dietrich \& Dmitriev 2003) and recent phylogenetic studies based on morphological (Dietrich 1999, Knight \& Webb 1993, Zahniser \& Dietrich 2008) and molecular data (Dietrich et al. 2001, Zahniser \& Dietrich 2010). These studies provided strong evidence

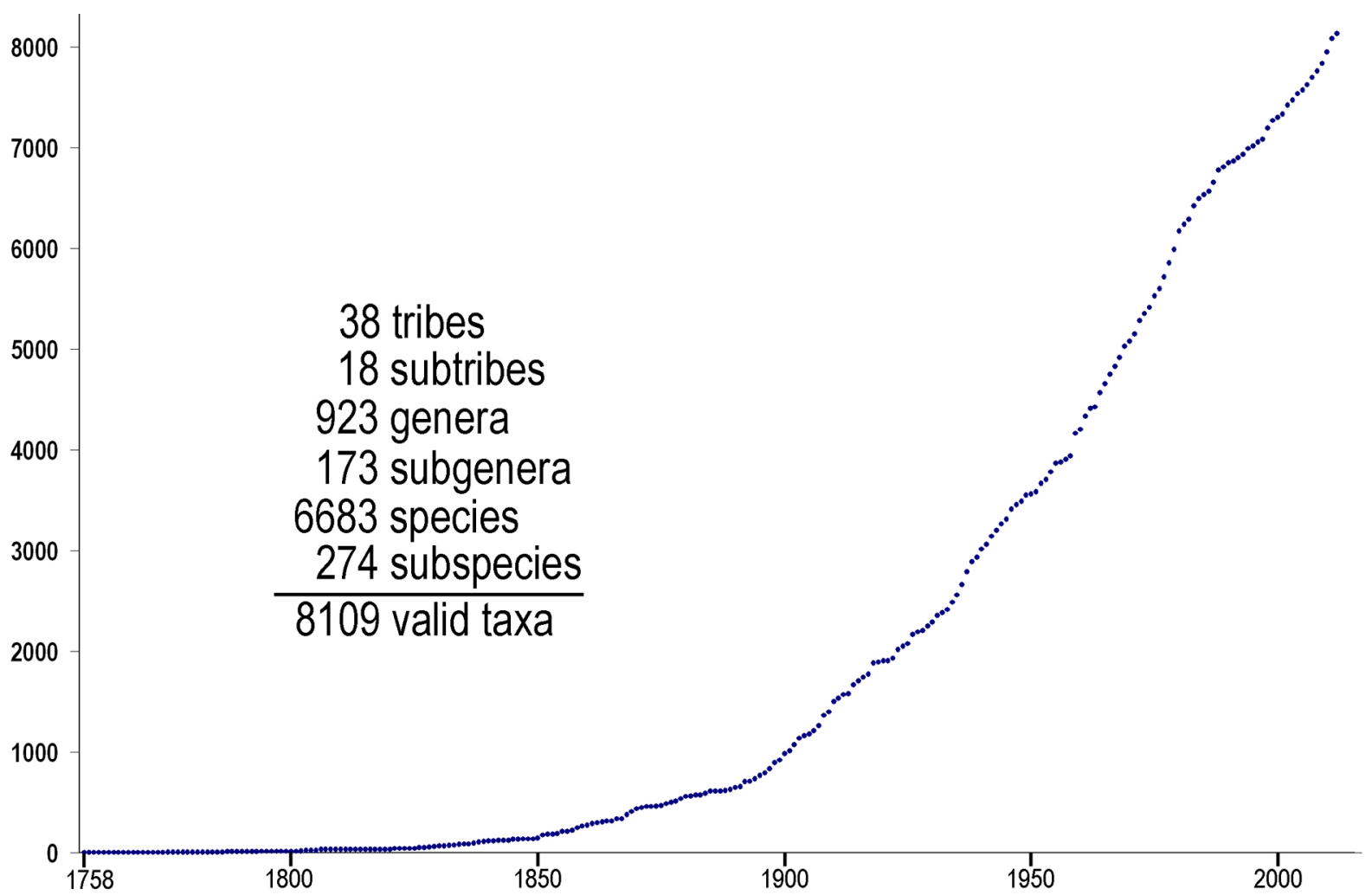

Fig. 1. Cumulative number of valid taxa (subspecies through subfamily) described 1758 to present. $\mathrm{X}$-axis indicates the year; Y-axis indicates cumulative number of valid taxa. 
that Deltocephalinae sensu Oman et al. (1990) was not monophyletic and that several other leafhopper subfamilies had their closest relatives within the deltocephaline lineage. Based on the accumulated data and understanding of the relationships of Deltocephalinae and other leafhoppers, Zahniser \& Dietrich (2010) adopted a revised, broader concept of the subfamily and provided a detailed history of the classification of the subfamily.

Despite the knowledge that has been gained in recent years, a comprehensive understanding of the relationships of all Deltocephalinae is far from complete. More taxa and more data need to be included in future phylogenetic studies to obtain a more resolved and supported phylogeny of the subfamily. In the current study, the taxon sample was increased over previous phylogenetic analyses by 37 taxa. The updated phylogenetic analyses presented here are used in combination with morphological examination of numerous representatives of Deltocephalinae to review all of the family-group taxa (tribes and subtribes) of Deltocephalinae and provide revised morphological descriptions, revised classifications, complete listings of genera included in each tribe and subtribe, and information on their phylogenetic relationships, classification status, ecology, vector species, and distribution. The revised classification clears up some unsatisfactory elements and oversights of previous classifications. It also proposes some changes that are supported in part by the most current phylogenetic analyses of the subfamily presented here. While more focused effort is needed to clarify some aspects of the classification, this represents a step forward toward a classification that reflects the phylogeny of the subfamily, as far as it is known.

Perhaps the most problematic aspects of the previous classification regard the 51 genera that were unplaced to tribe and the subfamily's largest tribe, Athysanini. The difficulty in the classification of these genera appears to be due to a rapid radiation of deltocephaline leafhoppers that left very little phylogenetic signal in morphological and molecular characters (Zahniser \& Dietrich 2010) among a morphologically diverse group of deltocephalines. They possess the typical $\mathbf{Y}$-shaped connective of the

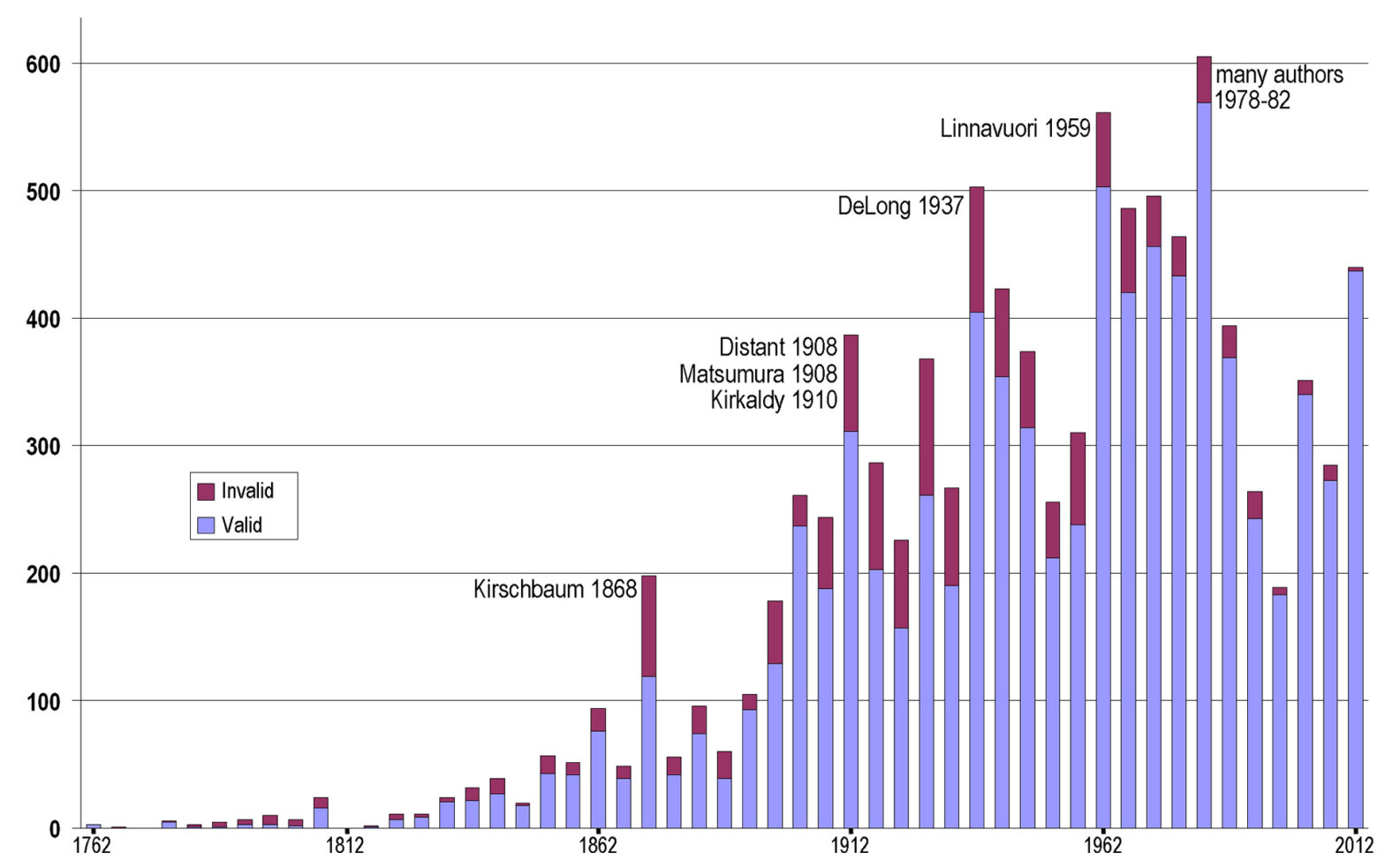

Fig. 2. Number of taxa described 1758 to present, summed over 5-year intervals. X-axis indicates the year; Y-axis indicates the number of valid (blue) and invalid (red) taxa described for the previous 5 years, inclusive of the given year. 
male genitalia but do not possess any of the characters that define other tribes, and are consequently a polyphyletic assemblage of genera that is difficult to make sense of as a whole. Effort was given here to separate some groups that have previously been placed in Athysanini or were unplaced to tribe, were recovered in the phylogenetic analyses here, and have diagnosable combinations of morphological characters which suggest that they form a monophyletic group. Several genera here retained in Athysanini are quite distinctive morphologically and may merit separate tribal status, given that they seem at least as "unusual" as the genera placed in monobasic tribes by previous authors (see Remarks under Athysanini below). However, we see little value in recognizing additional monobasic tribes until more detailed analyses can be conducted to further elucidate relationships within the group.

\section{I Online Interactive Key and Searchable Database}

This publication also serves to formally introduce the online searchable database and interactive key to tribes and subtribes of Deltocephalinae. These were produced using the 3I taxonomic database software package (Dmitriev 2006a) and are available through the first author's homepage $<$ http://imperialis. inhs.illinois.edu/zahniser/ $>$ and directly at the following links: Database Search $<$ http://imperialis.inhs. illinois.edu/zahniser/search.asp?key=Delt\&lng=En $>$ and Interactive Key to Tribes of Deltocephalinae: $<\mathrm{http} / /$ imperialis.inhs.illinois.edu/zahniser/key.asp?key=Delt\&lng=En\&i=1\&keyN=1>.

The database includes comprehensive nomenclatural data for all taxa of Deltocephalinae (Figs 1-2). This includes information for the 38 tribes, 18 subtribes, 923 genera, 173 subgenera, 6683 valid species, 274 subspecies, and 1458 invalid names of the subfamily. Each valid taxon has a designated taxon webpage that includes all of the information currently available for it in the database. All of the illustrations included in this publication are associated with online taxon pages for tribes, genera, and species. An additional 3800 illustrations of select genera and species are also included, with emphasis on the genera of Athysanini. Country-level distributional data taken from the literature are included for all species. The coverage is not exhaustive of all leafhopper literature but provides a baseline level of information on the distribution of species and higher taxa. The interactive key allows identification of the tribes and subtribes of Deltocephalinae using 56 morphological characters which were scored for all family-group taxa. The 3I database offers a number of other useful functions including advanced database searching options, automated taxon descriptions, and taxon and character comparisons. In the taxonomic treatments below, all taxonomic names are hyperlinked to their respective online taxon pages.

\section{Material \& Methods}

\section{Taxon Sampling for Phylogenetic Analysis}

The taxon sample used by Zahniser \& Dietrich (2010) was expanded here to include 37 additional exemplars of Deltocephalinae. All analyses except one supplementary analysis included 152 total taxa, 5 of which were putative outgroup taxa. The supplementary analysis included one additional taxon (Dorycephalus baeri Kouchakewitch, 1866) for which data were made available after branch support analyses had been performed on the previous taxon sample. Notable additions to the dataset include representatives of some family-group taxa (some of which are considered junior synonyms of other groups here) that were not included previously. Their previous classifications are given here if different from the revised classification proposed below (Results): Occinirvana eborea Evans, 1941 (Occinirvanini); Dwightla acutipennis Linnavuori \& Al-Ne'amy, 1983 (Dwightlini); Magnentius clavatus Singh-Pruthi, 1930 (Magnentiini); Anoterostemma ivanhofi Lethierry, 1876 (Limotettigini: Anoterostemmina); Proceps acicularis Mulsant \& Rey, 1855 (Scaphytopiini: Procepitina); Pseudophlepsius binotatus Signoret, 1880 (Opsiini: Eremophlepsiina); Allygidius abbreviatus Lethierry, 1878 (Athysanini: Allygidiina); Listrophora styx Linnavuori, 1979 (Eupelicini: Listrophorina). With these additions, all currently recognized tribes and subtribes of Deltocephalinae are represented in the data set except for Achaeticina (Opsiini) and Aglenina (Paralimnini). 
Table 1. A list of taxa included in the study with voucher numbers and GenBank accession numbers. The classification follows the revised classification proposed here. ${ }^{1}$ Fragments I, II, IV, and V from B. insula DeLong, 1964 and fragment III from B. ortha DeLong, 1937. ${ }^{2}$ Fragments I-III and V from

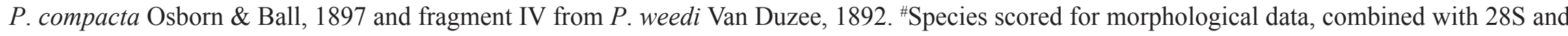
Histone H3 data from congeneric species. *Identification updated from previous study (Zahniser \& Dietrich 2010). **28S data taken from previous study (Dietrich et al. 2001; Rakitov, unpublished); vouchers are deposited at INHS.

\begin{tabular}{|c|c|c|c|c|c|c|}
\hline Tribe & Subtribe & Species & $28 \mathrm{~S}$ & Histone $\mathrm{H3}$ & Voucher \# & Locality \\
\hline Aphrodinae / Aphrodini & & Aphrodes bicincta & AF304579 & GU123794 & $71 * *$ & USA: Maryland \\
\hline Aphrodinae / Portanini & & Portanus sp. (undescribed sp.)* & AF304674 & -- & ** & Ecuador: Napo \\
\hline Aphrodinae / Portanini & & Portanus elegans ${ }^{\#}$ & -- & -- & n.a. & Venezuela \\
\hline Aphrodinae / Xestocephalini & & Xestocephalus desertorum & AF304619 & GU123892 & LH33** & USA: Illinois \\
\hline Aphrodinae / Xestocephalini & & Xestocephalus superbus ${ }^{\#}$ & -- & -- & n.a. & USA: Illinois \\
\hline Euacanthellinae & & Euacanthella palustris & GU123728 & GU123827 & DEL078 & Australia: NSW \\
\hline Neocoelidiinae & & Chinaia sp. (undescribed sp.)* & AF304676 & -- & lh104** & Ecuador: Napo \\
\hline Acinopterini & & Acinopterus acuminatus & JX845484 & GU123790 & DEL141 & USA: Illinois \\
\hline Acostemmini & & Acostemma stilleri* & GU123696 & GU123791 & DEL014 & Madagascar: Toliara Prov. \\
\hline Acostemmini & & Eryapus sp. & GU123727 & -- & DEL081 & Madagascar: Toliara Prov. \\
\hline Acostemmini & & Ikelibeloha cristata & JF835026 & JN177306 & LH177 & Madagascar: Province d'Antsiranana \\
\hline Acostemmini & & Iturnoria insulana & JN177307 & JN177308 & DEL132 & Madagascar: Province d'Antsiranana \\
\hline Arrugadini & & Arrugada affinis & GU123699 & GU123795 & HEC005 & Peru: Huanuco Prov. \\
\hline Arrugadini & & Arrugada rugosa ${ }^{\#}$ & -- & -- & n.a. & Bolivia: Yungas \\
\hline Athysanini & & Allygidius abbreviatus & JX845485 & JX845526 & DEL126 & France: Prov-Alpes-Côte d'Azur \\
\hline Athysanini & & Anoterostemma ivanhoffi & JX845487 & JX845528 & DEL130 & Scotland: Kirkcudbrightshire \\
\hline Athysanini & & Atanus sp. (undescribed sp.)* & GU123700 & GU123796 & DEL021 & Peru: Huanuco Prov. \\
\hline Athysanini & & Athysanus argentarius & GU123701 & GU123797 & DEL044 & USA: Illinois \\
\hline Athysanini & & Caranavia separata* & GU123710 & GU123807 & DEL073 & Peru: Junin Prov. \\
\hline Athysanini & & Cerrillus sp. & GU123711 & GU123808 & DEL058 & Peru: San Martín Prov. \\
\hline Athysanini & & Chimaerotettix ochrescens & JX845489 & JX845530 & DEL020 & Ecuador: Orellana \\
\hline Athysanini & & Colladonus lineatus & GU123718 & GU123815 & DEL052 & USA: California \\
\hline Athysanini & & Colladonus clitellarius $^{\#}$ & -- & -- & n.a. & USA: Illinois \\
\hline Athysanini & & Dagama forcipata & GU123720 & GU123817 & DEL055 & South Africa: W Cape Prov. \\
\hline Athysanini & & Euscelis seriphidii & GU123729 & GU123830 & DEL070 & Kyrgyzstan: Dzhalal-abad \\
\hline Athysanini & & Eusceloidea nitida & JX845494 & -- & DEL060 & Peru: San Martín Prov. \\
\hline Athysanini & & Eusama amanda & AF304590 & GU123829 & $77 \mathrm{~h}^{* *}$ & Mexico: Durango \\
\hline Athysanini & & Eutettix pictus & GU123730 & GU123831 & DEL100 & USA: Illinois \\
\hline Athysanini & & Idioceromimus delector & GU123740 & GU123844 & DEL065 & Brazil: Amazonas \\
\hline Athysanini & & Loralia sp. & GU123746 & GU123851 & DEL102 & Australia: South Australia \\
\hline Athysanini & & Napo sp. (undescribed sp.)* & GU123751 & GU123856 & DEL061 & Peru: San Martin Prov. \\
\hline
\end{tabular}




\begin{tabular}{|c|c|c|c|c|c|c|}
\hline Tribe & Subtribe & Species & $28 \mathrm{~S}$ & Histone $\mathrm{H3}$ & Voucher \# & Locality \\
\hline Athysanini & & Nesothamnus sanguineus & GU123755 & GU123860 & DEL072 & Ecuador: Orellana \\
\hline Athysanini & & Neohegira breviceps* & GU123753 & GU123858 & DEL077 & Peru: Huanuco Prov. \\
\hline Athysanini & & Neohegira sp. 075 & GU123786 & GU123891 & DEL075 & Peru: Huanuco Prov. \\
\hline Athysanini & & Orientus sp. & GU123757 & GU123862 & DEL022 & Taiwan: Taipei Co. \\
\hline Athysanini & & Orientus ishhidae \# & -- & -- & n.a. & USA: Illinois \\
\hline Athysanini & & Pachytettix sp. (undescribed sp.)* & GU123761 & GU123865 & DEL037 & Peru: Huanuco Prov. \\
\hline Athysanini & & Platymetopius obsoletus & GU123771 & GU123875 & DEL013 & Kyrgyzstan: Dzhalal-abad \\
\hline Athysanini & & Renonus rubraviridis & JX845524 & JX845552 & DEL127 & Mexico: Jalisco \\
\hline Athysanini & & Thamnotettix confinis & GU123783 & GU123888 & De1** & USA: Colorado \\
\hline Athysanini & & Thamnotettix sp. ${ }^{*}$ & -- & -- & n.a. & USA: California \\
\hline Athysanini & & Twiningia pellucida* & GU123785 & GU123890 & DEL053 & USA: California \\
\hline Athysanini & & Yungasia bidentata & GU123787 & GU123893 & DEL074 & Peru: Huanuco Prov. \\
\hline Bahitini & & Bahita sp. (undescribed sp.)* & GU123702 & GU123798 & DEL009 & Peru: Huanuco Prov. \\
\hline Bahitini & & Caruya sp.* & GU123703 & GU123799 & DEL099 & Peru: Huanuco Prov. \\
\hline Bahitini & & Kinrentius sp. & JX845523 & JX845549 & LH157 & Peru: Junín Prov. \\
\hline Bahitini & & Menosoma sp. ca. elegans & GU123749 & GU123854 & DEL101 & Peru: Huanuco Prov. \\
\hline Bahitini & & Oxycephalotettix tiputini & GU123759 & -- & DEL018 & Ecuador: Orellana \\
\hline Bahitini & & Taperinha adspersa & GU123780 & GU123885 & DEL032 & Peru: Huanuco Prov. \\
\hline Bonaspeiini & & Bonaspeia eriocephala & JX845521 & GU123804 & DEL049 & South Africa: W Cape Prov. \\
\hline Bonaspeiini & & Cerus goudanus & GU123712 & GU123809 & DEL050 & South Africa: W Cape Prov. \\
\hline Bonaspeiini & & Renosteria waverena & GU123772 & GU123878 & DEL048 & South Africa: W Cape Prov. \\
\hline Chiasmini & & Chiasmus varicolor* & GU123713 & GU123810 & Ah4** & Australia: NSW \\
\hline Chiasmini & & Chiasmus varicolor ${ }^{\#}$ & -- & -- & n.a. & Australia: NSW \\
\hline Chiasmini & & Doratura stylata & AF304589 & GU123821 & LH19** & USA: Maryland \\
\hline Chiasmini & & Driotura gammaroides & JX845492 & JX845533 & LH96 & USA \\
\hline Chiasmini & & Gurawa minorcephala & JX845495 & JX856131 & CHI066 & Thailand: Chiang Mai \\
\hline Chiasmini & & Listrophora styx & JX845500 & JX845539 & DEL138 & South Africa: Mpumalanga \\
\hline Chiasmini & & Nephotettix modulatus & GU123754 & GU123859 & CHI007 & Madagascar: Toliara Prov. \\
\hline Chiasmini & & Nephotettix sp. ${ }^{\#}$ & $\begin{array}{ll}- \\
\end{array}$ & -- & n.a. & Sri Lanka \\
\hline Chiasmini & & Protochiasmus mysticus* & GU123708 & GU123805 & DEL035 & Brazil: Goias \\
\hline Cicadulini & & Cicadula quadrinotata & GU123717 & GU123813 & DEL106 & Kyrgyzstan \\
\hline Cicadulini & & Cicadula melanogaster $\#$ & -- & -- & n.a. & USA: Illinois \\
\hline Cicadulini & & Elymana acuma & GU123726 & GU123826 & DEL092 & USA: Illinois \\
\hline Cicadulini & & Proceps acicularis & JX845511 & JX845550 & DEL110 & France: Prov-Alpes-Côte d'Azur \\
\hline Cicadulini & & Stenometopiellus sigillatus & JX845515 & -- & DEL064 & Kyrgyzstan: Chuy \\
\hline Cochlorhinini & & Ballana insula ${ }^{1}$ & AF304582-AF304580 & -- & LH52** & USA: California \\
\hline Cochlorhinini & & Ballana ortha ${ }^{1}$ & AF304581 & GU123801 & LH17** & USA: Wyoming \\
\hline Cochlorhinini & & Cochlorhinus pluto & AF304586 & GU123814 & LH08** & USA: California \\
\hline
\end{tabular}




\begin{tabular}{|c|c|c|c|c|c|c|}
\hline Tribe & Subtribe & Species & $28 \mathrm{~S}$ & Histone $\mathrm{H3}$ & Voucher \# & Locality \\
\hline Deltocephalini & & "DeltocephaAUS" (undesc. gen. \& sp.)* & GU123722 & GU123820 & DEL098 & Australia \\
\hline Deltocephalini & & Deltocephalus sp. & GU123721 & GU123819 & De18** & USA \\
\hline Deltocephalini & & Deltocephalus balli ${ }^{\#}$ & -- & -- & n.a. & USA: Illinois \\
\hline Deltocephalini & & Paramesodes sp. (undesc. sp.)* & GU123764 & GU123868 & DEL026 & Taiwan: Taichung Co. \\
\hline Deltocephalini & & Polyamia compacta ${ }^{2}$ & AF304609-AF304607 & GU123876 & $113 * *$ & USA: Colorado \\
\hline Deltocephalini & & ${\text { Polyamia weedi }{ }^{2}}^{2}$ & AF304608 & -- & LH90** & USA: Illinois \\
\hline Dorycephalini & & Dorycephalus baeri & JX845491 & JX845532 & HEC010 & Kyrgyzstan: Naryn \\
\hline Drabescini & Drabescina & Drabescus sp. & GU123724 & GU123824 & DEL028 & Taiwan: Taipei Co. \\
\hline Drabescini & Drabescina & Drabescus sp. ${ }^{\#}$ & -- & -- & n.a. & Nigeria: Ife Ife \\
\hline Drabescini & Paraboloponina & Bhatia satsumensis & GU123706 & GU123803 & DEL030 & Taiwan: Taipei Co. \\
\hline Drabescini & Paraboloponina & Parabolopona guttata* & GU123762 & GU123866 & DEL029 & Taiwan: Nantou Co. \\
\hline Drakensbergenini & & Drakensbergena retrospina & GU123725 & GU123825 & DEL051 & South Africa: KZN Prov. \\
\hline Eupelicini & Eupelicina & Eupelix cuspidata & AF304644 & GU123828 & $\operatorname{lh} 118^{* *}$ & Kyrgyzstan: Chuy \\
\hline Eupelicini & Paradorydiina & Paradorydium paradoxum* & AF304637 & GU123877 & lh116** & Kyrgyzstan: Dzhalal-abad \\
\hline Eupelicini & Paradorydiina & Chloropelix canariensis* & GU123715 & GU123811 & DEL071 & Portugal: Madeira Isl. \\
\hline Faltalini & & Hecullus bracteatus & GU123737 & GU123841 & HEC011 & USA: New Mexico \\
\hline Faltalini & & Kramerana junina & GU123743 & GU123848 & HEC004 & Peru: Junin Prov. \\
\hline Faltalini & & Tenucephalus sp. (undesc. sp.)* & GU123781 & GU123886 & HEC001 & Brazil: Goias \\
\hline Fieberiellini & & Fieberiella florii & AF304594 & GU123834 & LH23** & USA: Illinois \\
\hline Goniagnathini & & Goniagnathus guttulinervis & GU123736 & GU123838 & DEL002 & Kyrgyzstan: Dzhalal-abad \\
\hline Goniagnathini & & Goniagnathus rugulosus ${ }^{\#}$ & -- & -- & n.a. & Kyrgyzstan: Issyk-Kul \\
\hline Hecalini & Glossocratina & Glossocratus afzelii* & GU123735 & GU123837 & HEC009 & Madagascar: Toamasina Prov. \\
\hline Hecalini & Hecalina & Attenuipyga vanduzei & AF304653 & GU123822 & De26 & USA: Illinois \\
\hline Hecalini & Hecalina & Hecalus viridis & AF304596 & GU123840 & De27** & USA: Colorado \\
\hline Hypacostemmini & & Hypacostemma viridissima* & GU123739 & GU123843 & DEL094 & South Africa: KZN Prov. \\
\hline Koebeliini & Grypotina & Grypotes puncticollis \# & -- & -- & n.a. & USA: Pennsylvania \\
\hline Koebeliini & Grypotina & Grypotellus staurus* & $\begin{array}{l}\text { AF304651 } \\
\text { AF304652 }\end{array}$ & GU123839 & De33** & Greece: Delphi \\
\hline Koebeliini & Koebeliina & Koebelia grossa & AF304599 & GU123846 & LH56** & USA: California \\
\hline Limotettigini & & Limotettix striola & GU123745 & GU123850 & DEL004 & Kyrgyzstan: Osh \\
\hline Limotettigini & & Limotettix (Scleroracus) decumanus ${ }^{\#}$ & -- & -- & n.a. & Kyrgyzstan: Dzhalal-abad \\
\hline Luheriini & & Luheria constricta & JX845502 & GU123852 & DEL105 & Argentina: Salta \\
\hline Macrostelini & & Balclutha neglecta & GU123704 & GU123800 & De21** & USA \\
\hline Macrostelini & & Balclutha punctata $\#$ & -- & -- & n.a. & USA: Illinois \\
\hline Macrostelini & & Dalbulus gelbus & AF304587 & GU123818 & $\mathrm{D} 7 * *$ & USA: OH (OSU lab colony) \\
\hline Macrostelini & & Evinus peri & GU123731 & GU123832 & HEC012 & Kyrgyzstan \\
\hline Macrostelini & & Macrosteles quadrilineatus* & GU123748 & GU123853 & De22 & USA \\
\hline
\end{tabular}




\begin{tabular}{|c|c|c|c|c|c|c|}
\hline Tribe & Subtribe & Species & $28 \mathrm{~S}$ & Histone $\mathrm{H3}$ & Voucher \# & Locality \\
\hline Macrostelini & & Macrosteles quadrilineatus ${ }^{\#}$ & -- & -- & n.a. & USA: Illinois \\
\hline Magnentiini & & Magnentius clavatus & JX845503 & JX845541 & LH199 & India: Karnataka \\
\hline Mukariini & & Agrica arisana & GU123779 & GU123884 & DEL023 & Taiwan: Nantou Co. \\
\hline Mukariini & & Mukaria maculata & GU123750 & GU123855 & DEL024 & Taiwan: Changhua Co. \\
\hline Mukariini & & Mukaria sp. $^{\#}$ & -- & -- & n.a. & India: Assam \\
\hline Mukariini & & "Mukariini 146" (undesc. gen. \& sp.) & JX845505 & JX845543 & DEL146 & China: Shaanxi Prov. \\
\hline Mukariini & & Scaphotettix viridis & GU123774 & GU123879 & DEL068 & Taiwan: Tainan Co. \\
\hline Occinirvanini & & Occinirvana eborea & JX845507 & JX845545 & DEL143 & Australia: W. Aust. \\
\hline Opsiini & Circuliferina & Neoaliturus carbonarius & GU123752 & GU123857 & DEL012 & Kyrgyzstan: Dzhalal-abad \\
\hline Opsiini & Eremophlepsiina & Pseudophlepsius binotatus & JX845512 & JX845551 & DEL125 & Kyrgyzstan: Issyk-Kul \\
\hline Opsiini & Opsiina & Hishimonus phycitis & GU123738 & GU123842 & DEL031 & Taiwan: Taipei Co. \\
\hline Opsiini & Opsiina & Japananus hyalinus & JX845499 & JX845538 & DEL129 & USA: Illinois \\
\hline Opsiini & Opsiina & Nesophrosyne maritima & JX845506 & JX845544 & DEL109 & USA: Hawaii \\
\hline Opsiini & Opsiina & Opsius versicolor* & GU123756 & GU123861 & DEL043 & Israel: Negev \\
\hline Opsiini & Opsiina & Opsius stactogalus \# & -- & -- & n.a. & USA: Illinois \\
\hline Opsiini & Opsiina & Orosius orientalis & JX845509 & JX845547 & & Australia: New South Wales \\
\hline Opsiini & & $\begin{array}{l}\text { "Zambia122Ops" undescribed } \\
\text { genus near Libengaia }\end{array}$ & JX845520 & JX845560 & DEL122 & Zambia: Northwest Prov. \\
\hline Paralimnini & Paralimnina & Aflexia rubranura & GU123698 & GU123793 & De16** & USA \\
\hline Paralimnini & Paralimnina & Flexamia areolata & GU123733 & GU123835 & De38** & USA \\
\hline Paralimnini & Paralimnina & Laevicephalus monticola & GU123744 & GU123849 & $60 b^{* *}$ & USA \\
\hline Paralimnini & Paralimnina & Laevicephalus melsheimerii ${ }^{\#}$ & -- & -- & n.a. & USA: Illinois \\
\hline Paralimnini & Paralimnina & Paralimnus angusticeps & GU123763 & GU123867 & DEL001 & Kyrgyzstan: Issyk-Kul \\
\hline Pendarini & & Bandaromimus parvicauda & GU123705 & GU123802 & DEL076 & Peru: Huanuco Prov. \\
\hline Pendarini & & Chlorotettix galbanatus & -- & -- & n.a. & USA: Illinois \\
\hline Pendarini & & Chlorotettix rugicollis & GU123716 & GU123812 & DEL042 & USA: Illinois \\
\hline Pendarini & & Chlorotettix unicolor & GU123714 & -- & $138^{* *}$ & USA \\
\hline Pendarini & & Copididonus hyalinipennis & GU123719 & GU123816 & DEL007 & Brazil: Goias \\
\hline Pendarini & & Dorydiella kansana* & GU123723 & GU123823 & DEL003 & USA: Nebraska \\
\hline Pendarini & & Paraphlepsius nebulosus & GU123765 & GU123869 & DEL045 & USA: Illinois \\
\hline Pendarini & & Paraphlepsius irroratus ${ }^{\#}$ & -- & -- & n.a. & USA: Illinois \\
\hline Pendarini & & Tropicanus chiapasus & GU123784 & GU123889 & $89 a^{* *}$ & Mexico \\
\hline Pendarini & & Tropicanus flectus & JX845517 & JX845557 & DEL131 & Mexico: Chiapas \\
\hline Penthimiini & & Jafar javeti & JX845498 & JX845537 & DEL113 & Togo: Sotouboua Region \\
\hline Penthimiini & & Penthimia americana & AF304606 & GU123870 & LH34 & USA: Illinois \\
\hline Penthimiini & & Penthimidia eximia & JX845510 & JX845548 & DEL148 & Rep. of Congo: Iboubikro \\
\hline Penthimiini & & Penthimiola bella* & GU123766 & GU123871 & DEL080 & Madagascar: Toamasina Prov. \\
\hline Phlepsiini & & Excultanus conus & GU123732 & GU123833 & DEL005 & Mexico: Chiapas \\
\hline Phlepsiini & & Phlepsius intricatus & GU123768 & GU123873 & DEL017 & Kyrgyzstan: Dzhalal-abad \\
\hline
\end{tabular}




\begin{tabular}{|c|c|c|c|c|c|c|}
\hline Tribe & Subtribe & Species & $28 \mathrm{~S}$ & Histone H3 & Voucher \# & Locality \\
\hline Phlepsiini & & Korana rorulenta & GU123742 & GU123847 & DEL095 & Burkina Faso: Yako \\
\hline Scaphoideini & & Anoplotettix fuscovenosus & JX845486 & JX845527 & DEL147 & France: Provence-Alpes-Côte d'Azur \\
\hline Scaphoideini & & Loipothea sp. (undescribed sp.) & JX845501 & JX845540 & DEL115 & Zambia: Northwest Prov. \\
\hline Scaphoideini & & Mimotettix alboguttulatus & JX845504 & JX845542 & DEL117 & Zambia: Northwest Prov. \\
\hline Scaphoideini & & $\begin{array}{l}\text { Osbornellus sp. (undescribed sp. near } O \text {. } \\
\text { linnavuori DeLong)* }\end{array}$ & GU123758 & GU123863 & DEL033 & Peru: Huanuco Prov. \\
\hline Scaphoideini & & Phlogotettix cyclops & GU123769 & GU123874 & DEL027 & Taiwan: Ilan Prov. \\
\hline Scaphoideini & & Omanana sp. (undescribed sp.) & JX845508 & JX845546 & DEL128 & Mexico: Jalisco \\
\hline Scaphoideini & & Scaphoideus alboguttatus* & GU123773 & -- & DEL040 & Taiwan: Nantou Co. \\
\hline Scaphoideini & & Scaphoideus sp. SA & GU123775 & -- & DEL063 & South Africa: W Cape Prov. \\
\hline Scaphoideini & & Scaphoideus omani & JX845513 & JX845553 & DEL120 & Zambia: Northwest Prov. \\
\hline Scaphoideini & & $\begin{array}{l}\text { Scaphoidophyes sp. near pyrus Barnett \& } \\
\text { Freytag }\end{array}$ & JX845525 & JX845554 & DEL124 & Zambia: Copperbelt Prov. \\
\hline Scaphytopiini & & Scaphytopius frontalis & JX845514 & JX845555 & DEL116 & USA: Maryland \\
\hline Scaphytopiini & & Scaphytopius vaccinium & GU123776 & GU123880 & $1 \mathrm{lh} 09 \mathrm{~b}^{* *}$ & USA \\
\hline Scaphytopiini & & Scaphytopius acutus ${ }^{\#}$ & -- & -- & n.a. & USA: Illinois \\
\hline Selenocephalini & Adamina & Adama (Krisnella) elongata & GU123694 & GU123788 & DEL083 & Zambia: Copperbelt Prov. \\
\hline Selenocephalini & Adamina & Adama (Paracostemma) sp. & GU123697 & GU123792 & DEL015 & Rwanda: Nyungwe Forest \\
\hline Selenocephalini & Adamina & Adama (Paracostemma) sp. ${ }^{\#}$ & -- & -- & n.a. & Central African Republic: Boukoko \\
\hline Selenocephalini & Dwightlina & Dwightla acutipennis & JX845493 & JX845534 & DEL111 & Cameroon: Southwest Region \\
\hline Selenocephalini & Ianeirina & Abimwa knighti* & GU123695 & GU123789 & DEL104 & Zambia: Northwestern Prov. \\
\hline Selenocephalini & Ianeirina & Abimwa sp. ${ }^{\#}$ & -- & -- & n.a. & South Africa: KZN Prov. \\
\hline Selenocephalini & Selenocephalina & Citorus stipes & JX845490 & JX845531 & DEL144 & Zambia: Northwest Prov. \\
\hline Selenocephalini & Selenocephalina & Selenocephalus deserticola* & GU123777 & GU123881 & DEL041 & Israel: Negvev \\
\hline Stegelytrini & & Pachymetopius decoratus & GU123760 & GU123864 & DEL025 & Taiwan: Taichung Co. \\
\hline Stegelytrini & & Placidellus sp. & GU123770 & -- & $* *$ & \begin{tabular}{|l|} 
Thailand \\
\end{tabular} \\
\hline Stenometopiini & & Kinonia elongata & GU123741 & GU123845 & $75 \mathrm{~g} * *$ & USA \\
\hline Stenometopiini & & Stirellus catalinus & AF304614 & GU123882 & $82 \mathrm{k} * *$ & Mexico: Durango \\
\hline Stenometopiini & & Stirellus bicolor & -- & -- & n.a. & USA: Illinois \\
\hline Tetartostylini & & Tetartostylus parabolatus & GU123782 & GU123887 & DEL047 & South Africa: W Cape Prov. \\
\hline Vartini & & Stymphalus rubrolineatus & GU123778 & GU123883 & DEL062 & South Africa: W Cape Prov. \\
\hline--- & & "Peru059" undescribed gen.sp. & GU123767 & GU123872 & DEL059 & Peru: San Martin Prov. \\
\hline--- & & "Cameroon142" undescr. gen. \& sp. & JX845488 & JX845529 & DEL142 & Cameroon: Southwest Region \\
\hline--- & & "Ghana114" undescr. gen. \& sp. & JX845522 & JX845535 & DEL114 & Ghana: Western Region \\
\hline--- & & "Thailand112" undescr. gen. \& sp. & JX845516 & JX845556 & DEL112 & Thailand: Chaiyaphum \\
\hline--- & & "Zambia118" undescr. gen. \& sp. & JX845518 & JX845558 & DEL118 & Zambia: Northwest Prov. \\
\hline--- & & "Zambia119" near Phlogothamnus & JX845519 & JX845559 & DEL119 & Zambia: Northwest Prov. \\
\hline--- & & "Zambia121" near Houtbayana / Acacimenus & JX845496 & JX845536 & DEL121 & Zambia: Copperbelt Prov. \\
\hline
\end{tabular}


Taxa included in the analyses are listed in Table 1 which follows the revised classification provided here. GenBank accession numbers, voucher numbers, and locations are provided for all specimens included.

\section{Morphological and Molecular Data and Phylogenetic Analyses}

Morphological characters were the same as those used by Zahniser \& Dietrich (2010) but with the addition of two characters ( 9 and 75). The sequence of characters given here is different from that of Zahniser \& Dietrich (2010). Character names and states are listed in Appendix 1 and the data matrix is given in Appendix 2.

Methods for PCR amplification and sequence editing follow Zahniser \& Dietrich (2010). An alternative PCR primer for the D2 region of $28 \mathrm{~S}$ was used for some taxa (see Zahniser \& Nielson 2012). 28S rDNA sequences were aligned using the MUSCLE algorithm (Edgar 2004) implemented in MEGA5 (Tamura et al. 2011). The MUSCLE alignment was then slightly modified by hand in BioEdit 7.0.0 (Hall 1999). The final $28 \mathrm{~S}$ sequence alignment is available from the first author by request. Based on the alignment, a binary set of indel (insertion/deletion) characters was coded by hand. Alignment of the Histone H3 sequences did not require alignment algorithms.

Maximum parsimony (MP) analyses were run in PAUP* 4.0b10 (Swofford 1998). All characters were treated as unordered except characters 13,14, and 113, which were treated as ordered, and character 6 , which utilized a user-defined step matrix. 10,000 search replicates were performed with nchuck $=5$ and chuckscore $=1$, and the resulting set of trees were subsequently used as the starting tree set to search for all most parsimonious trees. 500 bootstrap (BS) replicates were performed with 100 pseudoreplicates per bootstrap replicate. Analyses were run to compute the decay index (DI = Bremer support) (Bremer 1988) for each node of the strict consensus of all most parsimonious (MP) trees. A search method similar to the original tree search was used to find MP trees with node constraints but with 1,000 initial search replicates due to computational time constraints. Total and average Bremer support for separate data partitions were calculated for each node.

Maximum likelihood (ML) analyses were run in GARLI 2.0 (Zwickl 2006). Separate partitions were used for the 28S, indel, each codon position of Histone H3, and morphology. Molecular models were explored for each molecular data partition using MrModeltest 2.2 (Nylander 2004). Results of the model tests for all partitions suggested the GTR $+\mathrm{I}+\mathrm{G}$ model except for the second codon position of Histone H3 for which the Jukes-Cantor model was selected. These selected models were implemented in ML analyses. The morphology and indel data sets were treated as "standard variable" data. The setting for the number of generations without significant change to terminate a replicate (genthreshfortopoterm) was set to $10,000.100$ search replicates were performed in the initial search. 100 bootstrap replicates were performed with 10 pseudoreplicates per bootstrap replicate. A separate ML analysis was performed with data for an additional taxon, Dorycephalus baeri Kouchakewitch, 1866. The analysis (50 search replicates) was performed without the morphological data set to examine the effect of excluding it in ML analysis; branch support was not assessed for this data set.

Bayesian analyses were run in MrBayes 3.2 (Ronquist et al. 2012). The GTR+I+G model was implemented for all molecular data partitions (28S and each codon position of Histone $\mathrm{H} 3$ ) as suggested by Huelsenbeck \& Rannala (2004) given the results of the model tests. 15 million generations were run with 2 runs and 4 chains per run. Trees were sampled every 1000 generations.

\section{Taxonomic Treatments}

The revised classification of the tribes provided below is based on the phylogenetic analyses presented here and on comparative morphological study of numerous genera of Deltocephalinae, representatives of which were borrowed from major museums. Although the ultimate goal is a classification that 
reflects phylogenetic relationships, this is difficult at present because many relationships remain poorly resolved by available data, and representatives of many genera have not been included in phylogenetic analyses; therefore, we chose a fairly conservative approach, in some cases retaining previously recognized groups even if they were not always recovered as monophyletic, pending further study. In general, changes to the higher classification (i.e., recognition of new tribes or re-definition of previously described tribes) are proposed only when supported at least in part by the phylogenetic results. Emphasis was also placed on the ability to diagnose any such groups with morphological characters. Thus, we did not establish tribes or subtribes for several groups of genera (clades) that received strong branch support in the phylogenetic results because we were unable to find diagnostic morphological characters to facilitate their identification. We hope that our results will stimulate further research not only to confirm their monophyly but also to determine whether such groups are diagnosable by as yet unrecognized morphological features. Along with specimens housed at INHS, specimens were borrowed from AMNH, OSUC, NMNH, BMNH, INSRB, RMCA, and MNHN (see acronyms below) with special attention to genera which were placed in the large tribe Athysanini, were unplaced to tribe in Deltocephalinae, or were perceived to be of questionable placement in their respective tribes. Representatives of most genera of Athysanini and genera unplaced to tribe in Deltocephalinae were examined for this study.

Tribe and subtribe descriptions were modified from the automated descriptions generated by the 3I database based on the characters that were scored for the interactive key to tribes and subtribes. In the descriptions of size, "small" = 2 to $4 \mathrm{~mm}$, "medium" = 4 to $8 \mathrm{~mm}$, and "large" = more than $8 \mathrm{~mm}$. The Geography and ecology sections are limited to notes on their distribution patterns in the major zoogeographical regions, their known host plants or general host plant use patterns, ecosystem types they are found in, and important species that are vectors of phytopathogenic diseases. Information on vector species was taken from Nielson (1968) and Weintraub \& Beanland (2006). The Selected references were chosen with emphasis on publications that are relatively recent (mostly within the past 50 years) and provide a broad perspective on the group, describe a significant number of new taxa, provide descriptions of the higher taxa, or are comprehensive treatments of the group over a relatively large geographical area.

One issue regarding the validity and authorship of some family group names must be addressed. A published abstract (Oman 1943) of P.W. Oman's Ph.D. thesis (Oman 1941) included the first validly published instances of names for the deltocephaline family-group taxa Acinopterini, Cochlorhinini, Dorycephalini, Mesamiini, Phlepsiini, Scaphoideini, and Scaphytopiini. Because Oman's published abstract did not include descriptions of these taxa or indicate which genera should belong in them, the names were not available when proposed according to ICZN Article 13.1. Although the published abstract referred to the taxon descriptions in the thesis, the thesis itself is not considered officially published according the ICZN (1999: Art. 8) which Article 13.1.2 requires. Nevertheless, some of the Oman (1943) names were taken by subsequent authors as valid (e.g., Oman 1949; Oman et al. 1990), and according to ICZN Article 13.2.1 those taken as valid should be treated as available from their original publication date. This article provides that family-group taxa first described after 1930 but before 1961 which do not satisfy the provisions of Article 13.1 are available if they were used as valid before 2000 and not explicitly rejected under Article 13. Zahniser \& Dietrich (2010) overlooked Article 13.2.1 and incorrectly treated the Oman (1943) names as unavailable nomina nuda. However, according to ICZN Article 13.2.1, the only family-group names published by Oman (1943) that are invalid (and unavailable) are Mesamiini and Phlepsiini because these names were not treated as valid in subsequent works published prior to 2000 .

Digital photographs of the habitus and face were taken with a Nikon D1x digital SLR camera or a Canon EOS Mark 5 DII configured with lenses by Microptics, Digital Lab XLT system. Digital photographs of 
the male and female genitalia were taken with a Q Imaging Micropublisher 3.3 digital camera mounted on an Olympus BX41 compound microscope. Multiple images were combined using the CombineZP software program (Hadley 2010). Photographs and stacked images were modified with Adobe Photoshop CS5. The combined photo and line drawing of the faces were produced in Adobe Illustrator CS5. All scale bars $=1.0 \mathrm{~mm}$.

For the taxon illustrations, the following standard structures and views are as follows: A, dorsal habitus; $\mathrm{B}$, lateral habitus; $\mathrm{C}$, face; $\mathrm{D}$, lateral view of male pygofer and terminal segments; $\mathrm{E}$, dorsal view of male pygofer and terminal segments; F, ventral view of subgenital plates and valve; G, ventral view of connective and style; $\mathrm{H}$, lateral view of aedeagus; I, caudal or ventral view of aedeagus. Additional notes and additional structures or illustrations are given in the figure captions as needed.

Acronyms for institutions used here are as follows:

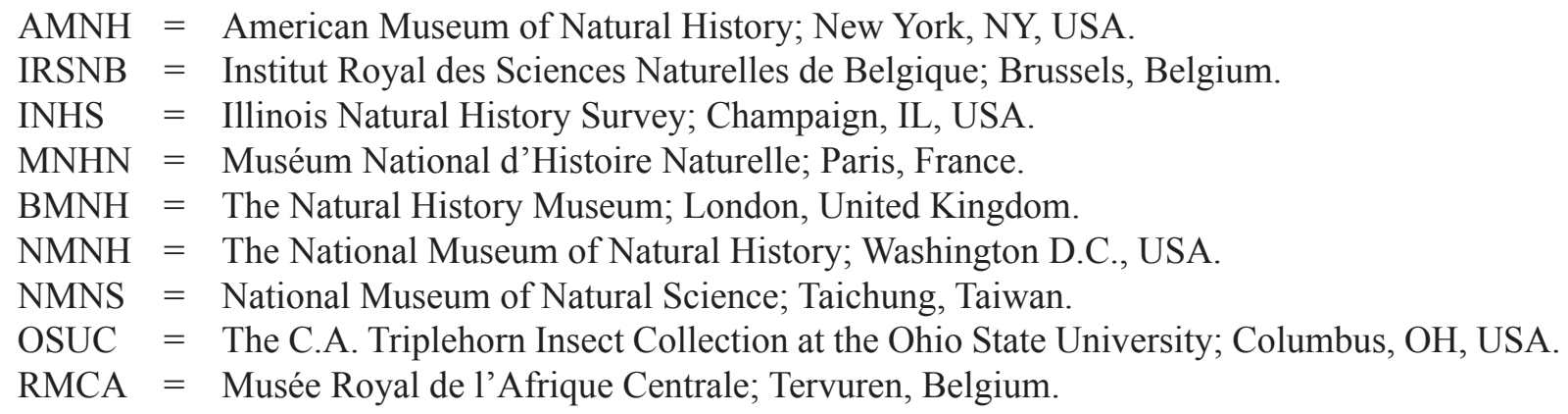

\section{Results}

\section{Phylogenetic Analyses}

A total of 803 parsimony-informative characters were included in the final data set. The entire aligned 28S rDNA data set included 3437 positions, and with segment II (see Dietrich et al. 2001) and the final $22 \mathrm{bp}$ excluded, the data set included 2908 characters; 500 of these were parsimony-informative, 402 were variable and parsimony-uninformative, and 2006 were constant. The indel data set included 60 characters, all of which were parsimony-informative. A total of $353 \mathrm{bp}$ of the Histone $\mathrm{H} 3$ nuclear protein coding gene were included; 122 characters were parsimony-informative, 8 were variable and parsimony-uninformative, and 223 characters were constant. All 121 morphological characters were parsimony-informative.

The parsimony analysis yielded 224 MP trees. The strict consensus is provided in Figs $3 \mathrm{a}$ and $3 \mathrm{~b}$ along with bootstrap scores and total and partitioned Bremer support values.

The ML tree with the best likelihood score was found in replicate 5 (of 100) and had a score of -45315.2484. Best scores from 16 other replicates were within 4 likelihood points of the best scoring tree, suggesting that the number of replicates performed here was sufficient in finding the most likely or nearly most likely trees. The tree with the best likelihood score is shown in Fig. 5 along with ML bootstrap scores. To allow for more space in the figure, the outgroup branches are shown separately in Fig. 4. The best tree in the analysis including Dorycephalus and excluding morphological data is shown in Fig. 6.

The 2 runs in the Bayesian analysis converged after 10 million generations, and the trees obtained prior to convergence were discarded as burnin. Convergence was determined by the random distribution of likelihood scores in a plot of the scores from the post-burnin generations. The ingroup branches of the 
Bayesian consensus tree are provided in Fig. 7, and the outgroup branching patterns and branch lengths are provided in Fig. 4, both with Bayesian posterior probability (PP) scores given below the branches.

\section{History of taxonomic descriptions in Deltocephalinae}

The compilation of the nomenclatural database allowed us to examine of some historical patterns in the taxonomy of Deltocephalinae, some of which are summarized in Figs 1 and 2. The first described deltocephaline was Psammotettix striatus (Linnaeus, 1758), originally placed in the genus Cicada.

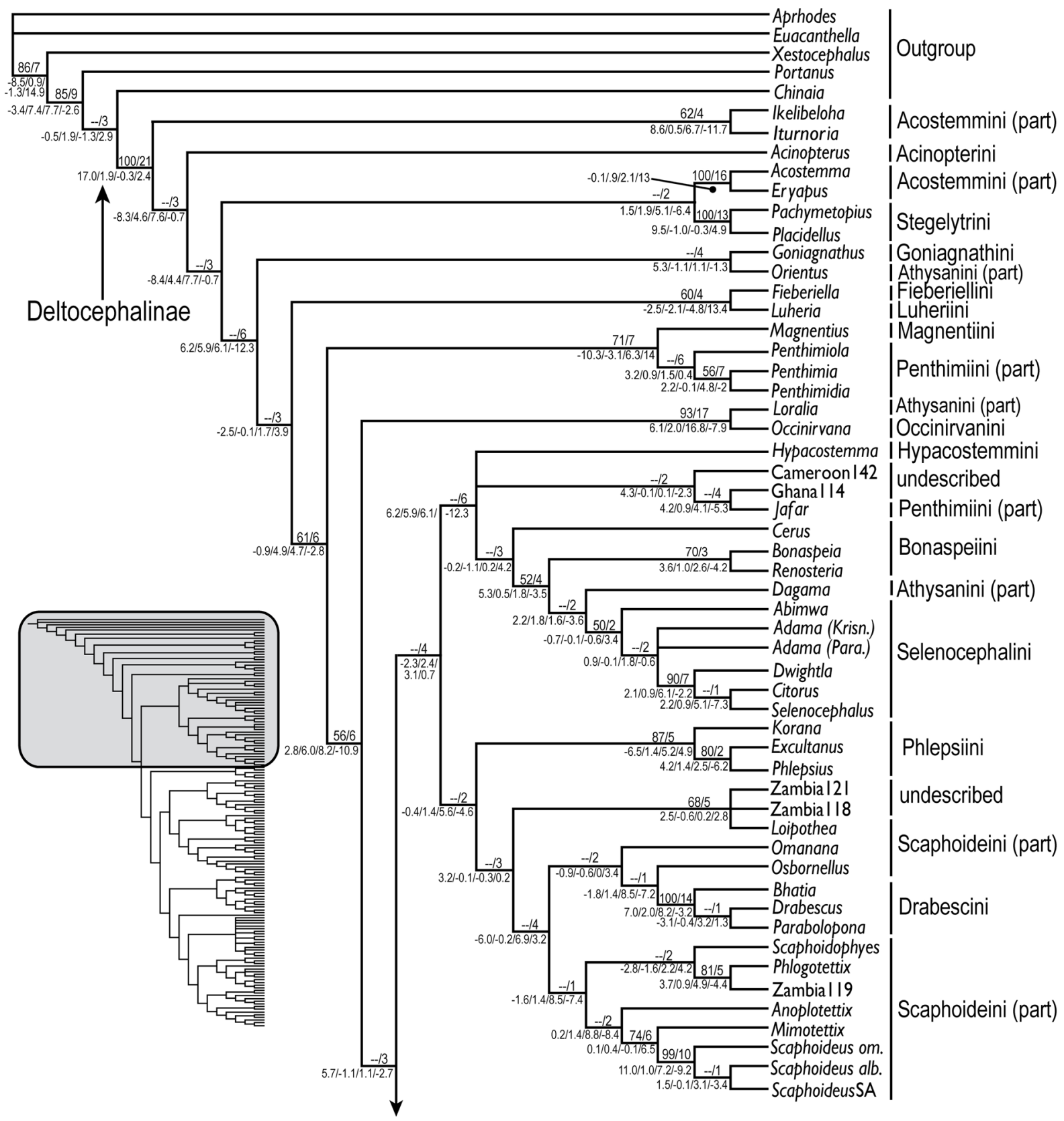

Fig. 3a. Top portion of the strict consensus of 224 MP trees. Numbers above branches are MP bootstrap / total Bremer support values. Numbers below branches are partitioned Bremer support values for 28S / indel / Histone H3 / morphology data sets. The revised classification is given to the right. 


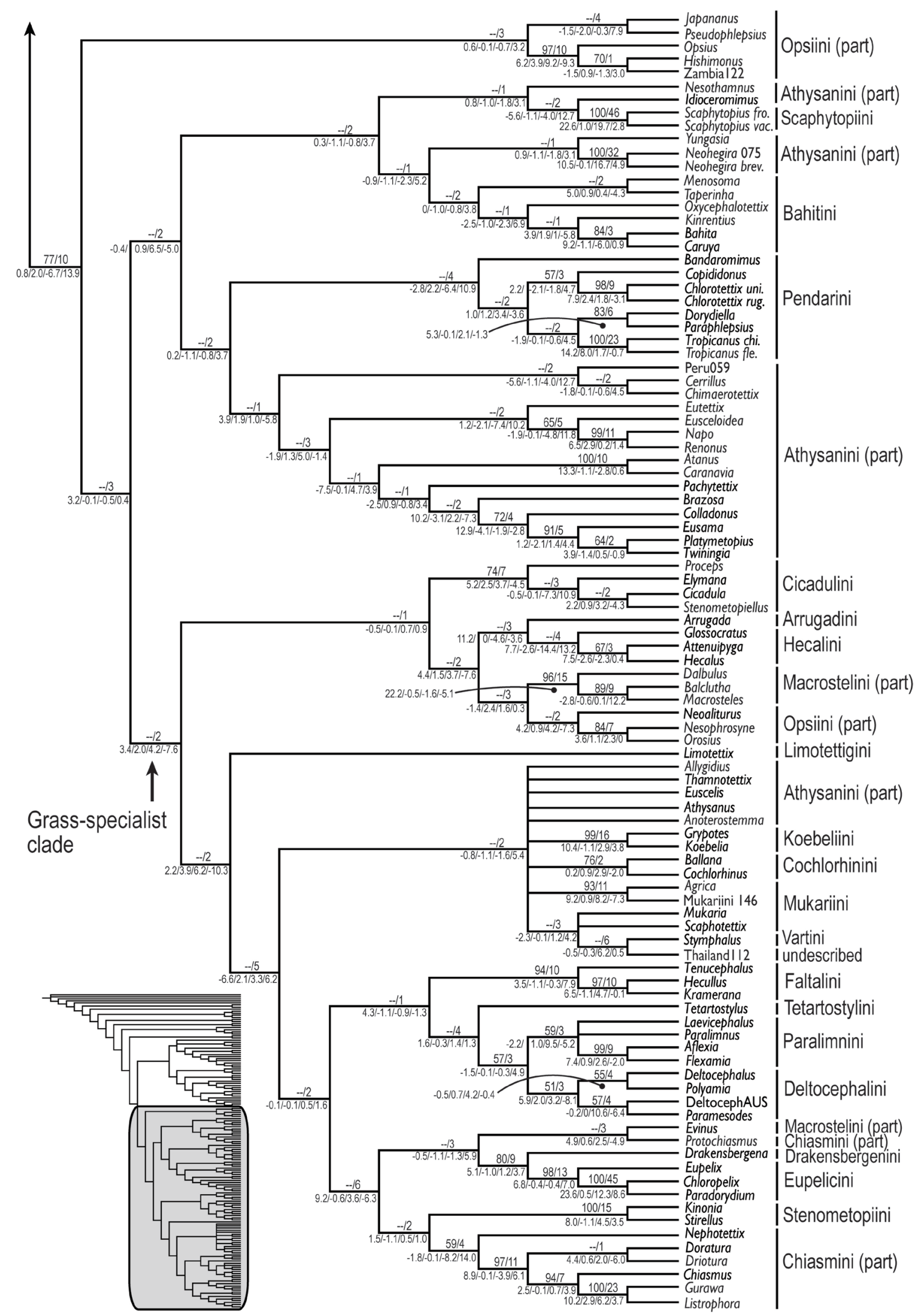

Fig. 3b. Lower portion of the strict consensus of 224 MP trees. Numbers on branches are as indicated for Fig. 3a. 
There were very few and scant descriptions of new taxa from 1758 through the mid-19th century, most of which were by the European workers Fabricius, Goeze, Panzer, Fallen, Germar, Zetterstedt, HerrichSchäffer, Burmeister, Curtis, Rambur and Boheman, and by the American, Thomas Say. The years 1850 to 1889 saw an increase in taxonomic descriptions, led again by European workers including Walker, Mulsant, Rey, Stål, Fieber, Kirschbaum, Motschulsky, Signoret, Flor, Fieber, Provancher, Lethierry and Signoret, and North and South American workers including Fitch, Uhler and Berg.

A marked increase in the description rate began in 1890 and has continued to the present day (Figs 1-2). A generation of late 19th century American workers including Van Duzee, Osborn, Ball, Gillette, Baker and Kirkaldy began to describe many New World Deltocephalinae. Other productive workers at the time included Matsumura, Melichar and Distant, who produced significant works on the fauna of India (e.g., Distant 1908, 1918). Dwight M. DeLong began publishing on Deltocephalinae in 1916 and continued until he passed away in 1984, leaving behind a rich 68-year legacy of work on the subfamily. $\mathrm{He}$ is distinguished as the most prolific author of species of Deltocephalinae, having named (usually as first or sole author) 833 valid species. DeLong's publication on Ballana (DeLong, 1937), in which 52 new species were described, contributed to high production during the years 1937-1941 (Fig. 2), and his publication on the leafhoppers of Illinois (DeLong, 1948) is still useful for identification of the North American fauna. The high productivity was also the result of the work by other pre-1950 20th century workers including Naudé, Lawson, Lindberg, Haupt, Kusnezov, Sleesman, Beamer, Ribaut, Ossiannilsson, Singh-Pruthi, Merino, Zachvatkin, Evans, Oman and Hepner. Rauno E. Linnavuori, the second most prolific describer of Deltocephalinae, began publishing on the group in 1951, and his high-quality revisions of regional faunas are still used today. Linnavuori's influence, especially beginning in 1959 with his revision of the Neotropical Deltocephalinae (Linnavuori 1959), along with that of DeLong and other authors (Dlabola, Vilbaste, Emeljanov, Heller, Kramer, Evans, Knight, Webb, Hamilton, Cheng, Remane, Asche, Cwikla, Blocker, Hicks, Whitcomb, Theron and Davies) made the years 1959 through 1988 the most prolific time period in the description of Deltocephalinae. This period was followed by a precipitous decline from 1989 to 1997 (although notable authors of this time include Meyer-Arndt, Remane, Zanol, Blocker, Viraktamath, Knight and Webb), but a resurgence over the last 15 years has been the result of the work of numerous authors including Viraktamath, Webb, Knight, Fletcher, Zhang, Wu Dai, Wei, Duan, Zanol, Stiller, Hamilton, Emeljanov, Dmitriev, Dietrich, Rakitov, Zahniser, Dominguez, Godoy, Li, Renhuai Dai and Xing.

A

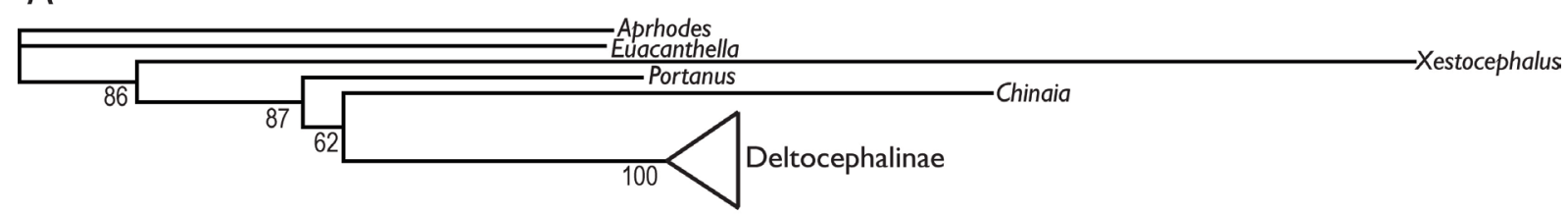

B

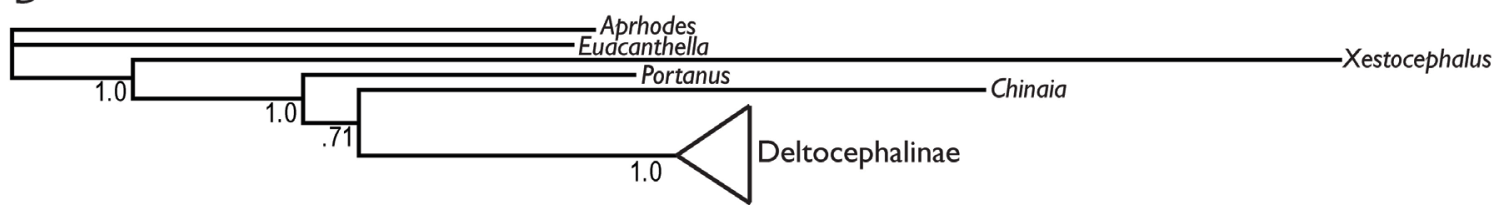

Fig. 4. A. Outgroup relationships inferred from ML analysis. Numbers below branches are ML bootstrap values. B. Outgroup relationships inferred from Bayesian analysis. Numbers below branches are PP values. 


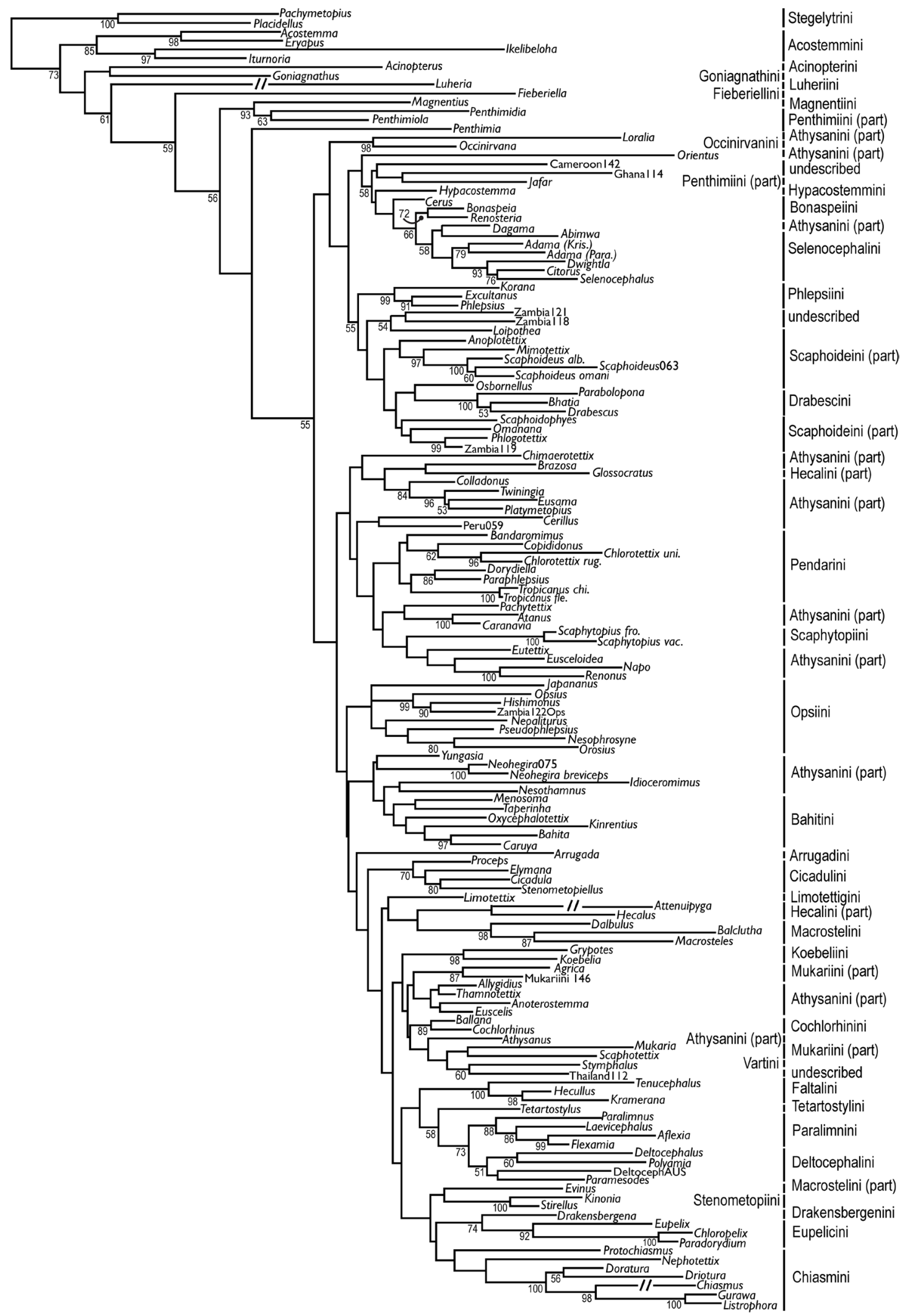

Fig. 5. Ingroup relationships inferred from ML analyses of combined data. Numbers below branches are ML bootstrap values. Shortened branches are broken with '// ' marks and are shown at half of their inferred lengths. 
ZAHNISER J.N. \& DIETRICH C.H., A review of the tribes of Deltocephalinae (Cicadellidae)

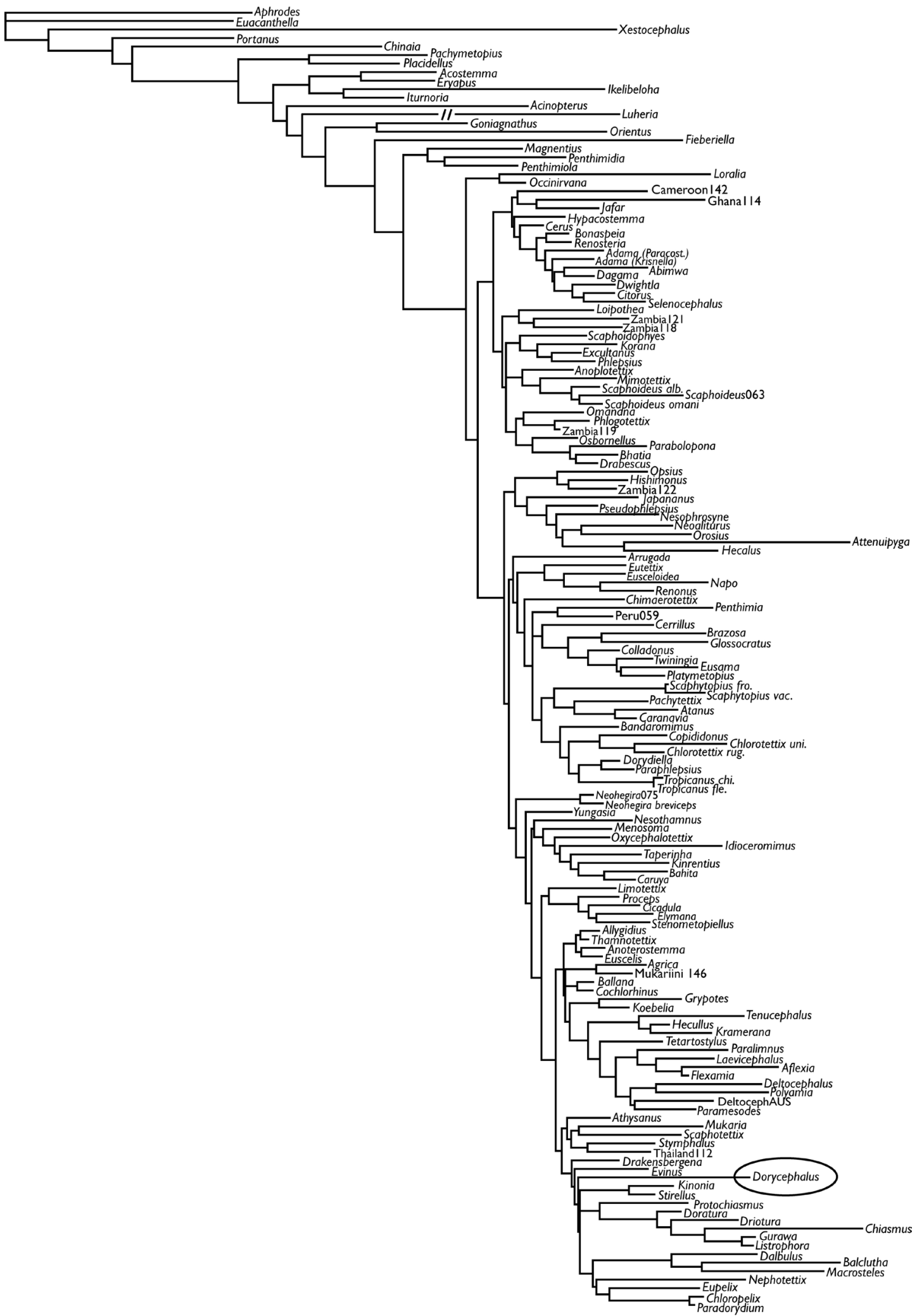

Fig. 6. ML tree inferred from analysis of molecular data only. This tree is included to illustrate the difference between including or excluding the morphological data from the phylogenetic analyses (see Fig. 5 for comparison of ML-inferred tree with morphological data). This analysis is the only one performed here to include Dorycephalus (Dorycephalini), and its position is highlighted. 


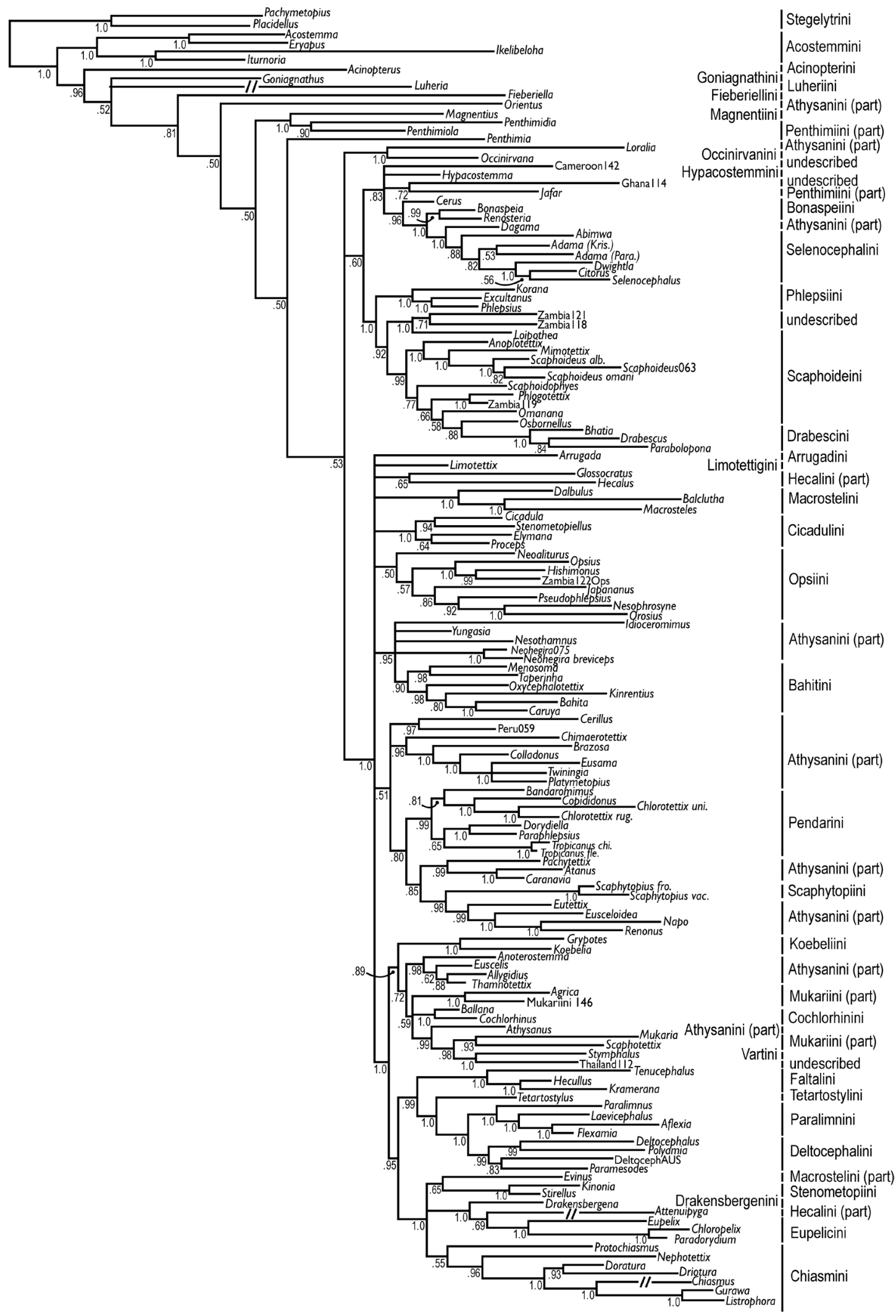

Fig. 7. Ingroup relationships inferred from Bayesian analysis of combined data. Numbers below branches are PP values. Shortened branches are broken with '//" marks and are shown at half of their inferred lengths. 
The most productive three years were 1959 (234 taxa), 1980 (189 taxa), and 1918 (159 taxa). The first and third most productive years were largely the result of monographic revisions by Linnavuori (1959) and Distant (1918), while the taxa were described by many authors in 1980. In general, the percentage of taxa described per year that are still valid (Fig. 2) has steadily increased, with the most recent two 5-year periods reaching 96 and $99 \%$. This could be due to a number of factors including better taxonomic descriptions in recent years, easier access to literature, or less time since their publication (thus, less chance to discover invalid names). The former two factors make it more likely that fewer potentially invalid names are currently being produced than was the case in earlier generations.

\section{Taxonomic Treatments}

A complete description of the subfamily Deltocephalinae was provided by Zahniser \& Dietrich (2010); an auto-generated description is available from the hyperlinked subfamily name below and a diagnosis of the subfamily is given below. Changes made to the classification are discussed in the Remarks section of each taxon treatment, along with discussion of the results of the phylogenetic analyses.

The revised concepts and new descriptions of tribes rely as much as possible on multiple independent morphological characters for their definition and diagnosis. Based on recent revisionary work and on the results of the recent phylogenetic analyses, it has become apparent that single morphological characters that sometimes previously defined tribes are not necessarily reliable predictors of a close relationship between genera. Similar character states have sometimes arisen multiple times independently in unrelated genera and are thus homoplasious. Conversely, a unique character state may have arisen once in the common ancestor of a lineage and subsequently be shared among some or most members of a monophyletic lineage but secondarily lost or transformed in other closely related members. The former case is illustrated by Scaphytopiini which was previously defined based on the wide gena visible behind the eye in dorsal view (usually in association with a narrow and produced head). The phylogenetic analyses here suggest that the genera previously included in Scaphytopiini (based only on this character) and included in the analyses (Japananus, Nesothamnus, Proceps, Scaphytopius and Stymphalus) are not closely related to one another. Thus, this character appears to be subject to homoplasy, and other characters need to be considered in the placement of these and other genera to tribe. An example of the latter case is illustrated in Chiasmini. This tribe was previously defined primarily by the unique hinged aedeagus. However Zahniser (2008a, 2011) pointed out several characters of the female ovipositor that were also helpful in defining the tribe and suggested a revised classification that included some genera with the ovipositor characters but without the hinged aedeagus (e.g., Leofa, Gurawa). This classification has been supported by molecular phylogenetic analyses (see below, Zahniser 2008b) and thus, in this case, the use of multiple characters was necessary to produce a classification that is supported by morphology and reflects phylogenetic relationships. The tribe is no longer diagnosable by a single character (i.e. the hinged aedeagus), but the character is still unique and occurs in most, but not all, Chiasmini. Thus, the use of multiple characters including external characters and those of the male and female genitalia strengthens our understanding of the phylogenetic relationships, character state variability, and generic constituency (taxonomy) of the tribe.

Deltocephalinae Dallas, 1870

\section{Note}

Because of the size and morphological diversity of this subfamily, nearly all of the characters listed below, which are the most common states in Deltocephalinae, occur in different states in some members of the subfamily. 


\section{Diagnosis}

Small to large, usually wedge-shaped leafhoppers. Head with ocelli on the anterior margin close to eyes; frontoclypeus not inflated, without carinae; lateral frontal sutures extending to ocelli; antennal ledges reduced or absent; gena with a fine erect seta laterad of lateral frontal suture; gena large, mostly concealing proepisternum. Forewing macropterous to brachypterous; if macropterous, with apices overlapping at rest; with 3 anteapical cells; often with 1 or more crossveins between A1 and claval suture; inner apical cell tapered distally, not extended to wing apex. Profemur AM1 seta distinct; row AV with short stout setae extending from base to $1 / 2$ to $2 / 3$ length of femur; intercalary row with numerous thin setae arranged in one row. Mesotrochanter with apical posteroventral stout seta. Metafemur macrosetal formula usually $2+2+1$ with penultimate pair close-set. Metatibia usually anteriorly-posteriorly compressed, ventral side with median ridge. Male pygofer with basolateral oblique membranous cleft. Valve produced posteriorly, lateral margins short, articulated with pygofer laterally. Subgenital plates articulated with each other and with valve; triangular, more or less depressed or flattened; with dorsal slot or fold articulating with style. Connective Y-shaped or 'linear' (with anterior arms closely appressed), rarely T-shaped (with arms widely divergent); without anteromedial lobe or process. Style broadly bilobed basally; apophysis not elongate. First valvula convex to relatively straight; dorsal sculpturing pattern reaching or not reaching dorsal margin; sculpturing pattern strigate, concatenate, reticulate, imbricate, maculose, or granulose. Second valvula with basal fused section as long as or longer than distal paired blades; with or without median dorsal tooth; usually with small to large, regularly or irregularly shaped dorsoapical teeth on apical $1 / 3$ or more; teeth sometimes restricted to apical $1 / 4$, or absent.

Included Tribes and Subtribes:

Acinopterini Oman, 1943

Acostemmini Evans, 1972

Arrugadini Linnavuori, 1965

Athysanini Van Duzee, 1892

Bahitini Zahniser \& Dietrich, 2013

Bonaspeiini Zahniser \& Dietrich, 2013

Chiasmini Distant, 1908

Cicadulini Van Duzee, 1892

Cochlorhinini Oman, 1943

Deltocephalini Dallas, 1870

Dorycephalini Oman, 1943

Drabescini Ishihara, 1953

Drabescina Ishihara, 1953

Paraboloponina Ishihara, 1953

Drakensbergenini Linnavuori, 1979

Eupelicini Sahlberg, 1871

Eupelicina Sahlberg, 1871

Paradorydiina Evans, 1936

Faltalini Zahniser \& Dietrich, 2010

Fieberiellini Wagner, 1951

Goniagnathini Wagner, 1951

Hecalini Distant, 1908

Glossocratina Dmitriev, 2002

Hecalina Distant, 1908

Hypacostemmini Linnavuori \& Al-Ne'amy, 1983

Koebeliini Baker, 1897

Grypotina Haupt, 1929 
Koebeliina Baker, 1897

Limotettigini Baker, 1915

Luheriini Linnavuori, 1959

Macrostelini Kirkaldy, 1906

Magnentiini Linnavuori, 1978

Mukariini Distant, 1908

Occinirvanini Evans, 1966

Opsiini Emeljanov, 1962

Achaeticina Emeljanov, 1962

Circuliferina Emeljanov, 1962

Eremophlepsiina Dmitriev, 2006

Opsiina Emeljanov, 1962

Paralimnini Distant, 1908

Aglenina Dmitriev, 2004

Paralimnina Distant, 1908

Pendarini Dmitriev, 2009

Penthimiini Kirschbaum, 1868

Phlepsiini Zahniser \& Dietrich, 2013

Scaphoideini Oman, 1943

Scaphytopiini Oman, 1943

Selenocephalini Fieber, 1872

Adamina Linnavuori \& Al-Ne'amy, 1983

Dwightlina McKamey, 2003

Ianeirina Linnavuori, 1978

Selenocephalina Fieber, 1872

Stegelytrini Baker, 1915

Stenometopiini Baker, 1923

Tetartostylini Wagner, 1951

Vartini Zahniser \& Dietrich, 2013

\section{Key to Tribes}

1. Crown sharply produced anteriorly (Fig. 48A); head narrower than pronotum; gena not incised laterally below eye (Fig. 48C), visible behind eye in dorsal view (Fig. 48A); frontoclypeus elongate (Fig. 48C); forewing with numerous reflexed costal veins (Fig. 48B); connective a transverse bar with two lateral arms directed obliquely laterad, stem absent (Fig. 48G); usually with pair of long processes articulated with connective (Fig. 48G, J); Nearctic, Neotropical .... Scaphytopiini

- Without the above combination of characters; crown variable; head width variable; gena incised laterally below eye (exceptions: some Acostemmini, Opsiini, Stegelytrini, Scaphoideini, and Vartini); forewing with or without reflexed costal veins; connective not as above; usually without long processes articulated with connective (exceptions: Adamina, some Scaphoideini)

2. Connective T-shaped, bar-shaped, or platelike or, if Y-shaped, with arms forming broadly obtuse angle (e.g., Figs 8G, 25G, 33G, 53G); style more or less linear, not broadly bilobed at base (e.g., Figs $8 \mathrm{G}, 25 \mathrm{G}, 33 \mathrm{G}, 53 \mathrm{G})$

- Connective with anterior arms somewhat or not at all divergent, connective $\mathbf{Y}$ - or $\mathbf{U}$-shaped (e.g., Figs $11 \mathrm{G}, 52 \mathrm{G}$ ) or 'linear' with anterior arms closely appressed (e.g., Fig. 17G); style broadly bilobed at base (e.g., Fig. 11G)

3. Head much narrower than pronotum; profemur row AM with numerous preapical macrosetae; metatibia dorsal surface with supernumerary macrosetae between rows $\mathrm{PD}$ and $\mathrm{AD}$, especially 
basally; pro- and mesotibia row PD and often row $\mathrm{AD}$ with numerous $(>6)$ macrosetae; forewing appendix often large, extending around apex; Palearctic, Oriental ....................................Stegelytrini

- Head slightly narrower to wider than pronotum; profemur row AM with not more than 2 preapical macrosetae; metatibia dorsal surface without supernumerary macrosetae; pro- and mesotibia with $<6$ macrosetae; forewing appendix reduced or absent

4. Anterior margin of head usually with a single distinct transverse carina between ocelli; epistomal suture keeled (Fig. 9C) or evident from change in texture on frontoclypeus; subgenital plates completely or partially fused to each other and/or to valve (if completely articulated then pronotum produced dorsally, with longitudinal crest, Ikelibeloha); Madagascar Acostemmini (part)

- Anterior margin of head shagreen, glabrous, or with several irregular striae or carinae, without single distinct carina between ocelli; epistomal suture not keeled; subgenital plates not fused, articulated with valve

5. Forewing acuminate apically (Fig. 8B); head narrower than pronotum (Fig. 8A) (exception: Cariancha); connective bar-shaped or plate-like, without well-defined stem (Fig. 8G); Nearctic, Neotropical Acinopterini

- Forewing rounded apically; head as wide as or wider than pronotum; connective with welldeveloped anterior arms posterior stem

6. Connective with median, darkly sclerotized $\mathbf{Y}$ - or $\mathbf{T}$-shaped ridge following stem and arms of connective (Fig. 25G); forewing central anteapical cell not strongly constricted medially; female pregenital sternite VIII not concealed and folded beneath sternite VII, movably articulated with sternite VII(Fig. 25J); Palearctic, Nearctic (Fieberiella florii introduced in North America) ...........Fieberiellini

- Connective evenly sclerotized throughout (Fig. 33G); forewing central anteapical cell constricted medially (Fig. 33B); female pregenital sternite VIII folded beneath and concealed by sternite VII, sternites not articulated with each other; Neotropical Luheriini

7. Subgenital plates fused to each other (Figs 9F, 26F); valve straplike, fused to pygofer laterally (Fig. 9D, F) OR not readily apparent (fused to plates); connective fused with aedeagus (Figs 9H, 26H)

- Subgenital plates usually free; valve present, articulated with pygofer laterally and usually articulated with subgenital plates; connective articulated with or fused to aedeagus; rarely with plates fused and connective fused with aedeagus, and if so then body slender or relatively small in size, clypellus tapering apically (Deltocephalini: Miradeltaphus, Yuanamia)

8. Anterior margin of head with a distinct transverse carina between ocelli; forewings tectiform, not overlapping at rest (Fig. 9A); forewing appendix reduced or absent (9B); valve strap-like, fused to pygofer laterally (Fig. 9D, F); Afrotropical (especially diverse in Madagascar), Oriental (India) Acostemmini (part)

- Anterior margin of head without transverse carina; forewing, if macropterous, with apices overlapping at rest (Fig. 26A) and appendix extending around wing apex (Fig. 25A); valve not readily apparent, fused to subgenital plates; Palearctic, Afrotropical, Oriental, and Australian Goniagnathini

9. Head strongly produced, elongate or spatulate (Figs 18A, 21A, 22A, 23A); gena acutely incised laterally below eye (Figs 18C, 21C, 22C, 23C); profemur intercalary row absent or with few scattered setae; metafemur macrosetal formula $2+0$ or $2+1$; metatarsomere I shorter than metatarsomeres II and III combined, expanded apically; metatibia straight throughout length and distinctly bent distally in dorsal view 
- Without above combination of characters; head produced or not; gena obtusely incised (e.g., Fig. 11C) or not incised laterally below eye; profemur intercalary row more or less welldelimited and with several to many long fine setae; metafemur macrosetal formula usually $2+2+1$; metatarsomere I longer than metatarsomeres II and III combined, usually not expanded apically; metatibia slightly arcuate throughout its length in dorsal view . .15

10. Aedeagus movably hinged between base and shaft (Fig. 14H); connective with anterior arms closely appressed (Fig. 14G); first valvula dorsal sculpturing maculose to granulose, submarginal; second valvula teeth obtusely triangular, serrate, uniformly shaped; Old World .... Chiasmini (part, Listrophora, some Chiasmus)

- Aedeagus without hinge; connective with anterior arms separate (Y-shaped); first valvula dorsal sculpturing pattern variable; second valvula teeth variable

11. Ocelli on crown

- Ocelli on anterior margin of head

12. Macropterous; crown encroaching onto eyes (Fig. 22A-C); frontoclypeus with median longitudinal carina (Fig. 22C); Palearctic ........................................Eupelicini (part, Eupelicina)

- Brachypterous (Fig. 21A-B); crown not encroaching onto eyes; frontoclypeus and clypellus tumid, without longitudinal carina (Fig. 21B-C); Afrotropical (South Africa, Lesotho)

Drakensbergenini

13. Macropterous; hindwing submarginal vein evanescent apically; valve fused to pygofer (Fig. 23D); male pygofer without basolateral cleft (Fig. 23D); style base connected or articulated only laterally with apical part (Fig. 23J); Old World Eupelicini (part, Paradorydiina)

- Brachypterous or macropterous, hindwing submarginal vein complete; valve articulated to pygofer; male pygofer with basolateral cleft; style base broadly fused with apical part (e.g., Fig. 27G) ........14

14. Aedeagus with terminal processes (e.g., Fig. 27H-I); first and second valvulae humpbacked dorsally; first valvula with distinctly delimited ventroapical sculptured patch; second valvula without teeth; Nearctic Hecalini (part, Attenuipyga, Neoslossonia)

- Aedeagus without terminal processes (Fig. 18H-I); first and second valvulae not humpbacked dorsally; first valvula without distinctly delimited ventroapical sculptured patch; second valvula with distinct teeth; Palearctic Dorycephalini

15. Anterior margin of pronotum exceeding eyes in dorsal view (Fig. 35A); integument black, shiny, punctate (Fig. 35A-B); Afrotropical, Oriental Magnentiini

- Anterior margin of pronotum not exceeding eyes in dorsal view; integument rarely completely black and punctate

16. Antennal ledge strong, forming distinct ledge, sometimes seemingly continuous with anterior margin of head (Figs 19C, 45C); protibia dorsal side flat, margins carinate; forewing appendix

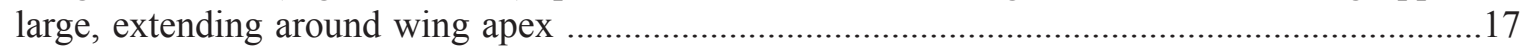

- Antennal ledge absent or represented by weak carina not forming ledge; protibia rounded dorsally; forewing appendix usually smaller, not extending around wing apex .18

17. Large, robust leafhoppers, not squat or dorsally rounded or ventrally flattened (Fig. 19A-B); ocelli on anterior margin of head (19C); frontoclypeus texture longitudinally striate or rugose (Fig. 19C); Palearctic, Ethiopian, Oriental, Australian ...Drabescini (part, Drabescina) 
- Robust and squat, dorsally rounded, ventrally flattened leafhoppers (Fig. 45A-B); ocelli on crown distant from eyes (Fig. 45B); frontoclypeus texture glabrous or finely shagreen; Cosmopolitan ..

Penthimiini

18. Crown and face strongly rugose (Fig. 10A-C); body greater than $7 \mathrm{~mm}$ in length; Neotropical

Arrugadini

- Crown and face shagreen, glabrous, or striate, not rugose, or if rugose then body less than $7 \mathrm{~mm}$ in length

19. Aedeagus bifurcate, with 2 gonopores

- Aedeagus with one shaft and gonopore

20. Face (Fig. 36B) strongly depressed, nearly horizontal, sometimes concave posteroventrally in profile, sometimes inflated anterodorsally; anterior margin of head often with one or more carinae or sharp ridges; antennal bases near anterodorsal (upper) corners of eyes (Fig. 36C); stem of connective short; Afrotropical, Oriental ...................................................ukariini (part)

- Face oblique, not strongly depressed, not concave in profile; anterior margin of head without carinae (foliaceous in Chlidochrus); antennal bases near middle or posteroventral (lower) corners of eyes; stem of connective longer; Cosmopolitan

Opsiini

21. Head often somewhat produced (Fig. 36A); face flattened or frontoclypeus inflated medially or anteriorly, lying in horizontal plane in lateral view (Fig. 36B-C); antennal bases near anterodorsal (upper) corners of eyes (Fig. 36C); eyes large (Fig. 36C); antennae often long, longer than width of head; profemur row AV setae absent; outer anteapical cell often confluent with or nearly touching costal vein (Fig. 36B); Oriental, feeding on bamboo Mukariini (part)

- Without above combination of characters; head produced or not; face flattened or not, not distinctly inflated anteriorly, lying in horizontal plane in lateral view or not; size of eyes and antennae variable; profemur row AV setae present or absent; outer anteapical cell usually not touching or confluent with costal vein

22. Connective Y-shaped and fused to aedeagus (Fig. 16G-H); male pygofer with one or more pairs of teeth, spines, or processes (Fig. 16D-E); male pygofer side excavated medially, scoop-like (Fig. 16E); male segment $\mathrm{X}$ long, sclerotized laterally, sometimes sclerotized ventrally and/or dorsally (Fig. 16D-E); second valvulae with a median dorsal tooth; Nearctic, Neotropical (Cochlorhinus pluto introduced in Chile) Cochlorhinini

- Connective shape variable, but not Y-shaped and fused to aedeagus; male pygofer with or without processes; male pygofer side variable; male segment $\mathrm{X}$ variable; second valvulae with or without dorsal tooth on shaft

23. Metatarsomere I with platellae on plantar surface; ocelli distant from eyes, at least $4 \mathrm{x}$ own diameter from adjacent eye (e.g., Fig. 30C)

- Metatarsomere I without platellae on plantar surface; ocelli usually close to eyes (exceptions: some Athysanini, Chiasmini, and Faltalini)

24. Head not produced anteriorly (Fig. 30A); crown rounded to face (Fig. 30B) or anterior margin of head carinate, not foliaceous; Palearctic, Oriental, Nearctic (Grypotes puncticollis introduced to northeastern North America) Koebeliini, in part (Grypotina)

- Head strongly produced, foliaceous anteriorly (Figs 31A-C, 37A-C) ……………………....25

25. Ocelli on face (Fig. 31C); antennae not very long; forewing veins pustulate, appendix absent (Fig. 31A-B); Nearctic (western North America) Koebeliini (part, Koebeliina) 
- Ocelli on crown (Fig. 36A1-A2); antennae very long; forewing veins not pustulate, appendix present; Australian (Western Australia) Occinirvanini

26. Color often greenish or bluish with red or orange longitudinal stripes (Fig. 56A-B); crown produced, pointed (Fig. 56A); gena not incised laterally below eye, visible behind the eye in dorsal view (Fig. 56B-C); frontoclypeus elongate (Fig. 56C); profemur intercalary row setae thick and extending to or basad of middle of profemur; forewing macropterous, truncate apically (56B); apodemes of male sternite II long, subrectangular, flared apically, directed posterolaterally (Fig. 56J); connective with anterior arms closely appressed (Fig. 56G); male segment X tube-like, protruding from pygofer, often well-sclerotized (Fig. 56D-E, K); Afrotropical, Oriental .......................Vartini

- Color variable; crown produced or not; gena usually incised laterally below eye, not visible behind eye in dorsal view (exception: some Scaphoideini); frontoclypeus variable, usually not very elongate; profemur intercalary row setae fine; if macropterous, forewing usually not truncate apically; apodemes of male sternite II not as above; connective variable; male segment $\mathrm{X}$ variable

27. Clypellus parallel-sided or tapering apically (e.g., Fig. 17C); connective linear, triangular, or racquetlike in shape, with anterior arms closely appressed anteriorly (e.g., Figs 17G, 42G)

- Clypellus variable; connective usually Y-shaped (e.g., 54G) or U-shaped (e.g., Fig. 52G), with anterior arms separated (exception: some Athysanini, Scaphoideini) .....

28. Subgenital plates fused with valve and apex of plate with sharply pointed process (Fig. 55F); connective articulated posteriorly with a pair of long paraphyses (Fig. 55G); female sternite VII ovate, sometimes very long; Palearctic, Afrotropical .........................Tetartostylini

- Subgenital plates usually articulated with valve, apices without sharply pointed processes; paraphyses present or absent; female sternite VII not ovate, not very long ................................29

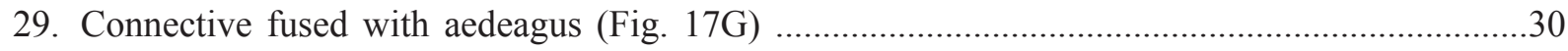

- Connective articulated with aedeagus (Fig. 42G) ...................................................................

30. Body elongate, somewhat depressed; anterior margin of head transversely striate or carinate; first valvula dorsal sculpturing pattern maculose to granulose and distinctly submarginal, with distinctly delimited ventroapical sculptured area; second valvula without dorsal teeth; ovipositor protruding well beyond pygofer apex; Nearctic (Mexico), Neotropical ...............Faltalini (part, Tenucephalus)

- Body not elongate or depressed; crown rounded to face, anterior margin shagreen; first valvula sculpturing pattern imbricate (scale-like) and reaching dorsal margin, without distinct apicoventral sculptured area; second valvula with irregular teeth; ovipositor not protruding far beyond pygofer; Cosmopolitan Deltocephalini

31. Aedeagus movably hinged between base and shaft (Fig. 14H) (exceptions: Baileyus, some Gurawa, Paraphrodes); ovipositor protruding beyond pygofer apex (exceptions: Gurawa, Baileyus, Omaranus, Paraphrodes); first valvula dorsal sculpturing pattern maculose to granulose, submarginal; second valvula teeth uniformly shaped, obtusely triangular, serrated; Cosmopolitan Chiasmini (part)

- Aedeagus entire, not movably hinged; ovipositor not protruding far beyond pygofer; first valvula dorsal sculpturing pattern usually imbricate, reaching dorsal margin; second valvula teeth not uniformly obtuse and triangular, serrated or not; Cosmopolitan Paralimnini

32. Macropterous, forewing with 2 anteapical cells (Fig. 34B); usually small, slender leafhoppers (Fig. 34A-B); Cosmopolitan Macrostelini

- Macropterous to brachypterous, if macropterous then usually with 3 anteapical cells; if with 2 anteapical cells, then larger, more robust leafhoppers .33 
33. Small to medium sized, slender, elongate leafhoppers (Fig. 15A); usually yellow, ochraceous, or pale green, often with paired black or brown markings or spots on head (Fig. 15A-C); subgenital plate often with row of macrosetae distant from lateral margin, with numerous long fine setae laterally (Fig. 15F); male pygofer incised dorsally nearly to base (Fig. 15D-E); male segment X elongate and sclerotized dorsally (Fig. 15D-E) (not elongate in Knullana DeLong); Palearctic, Nearctic, Neotropical Cicadulini

- Size and color variable; subgenital plate macrosetae variable, with or without long fine setae; male pygofer dorsal incision variable; male segment $\mathrm{X}$ usually not elongate and strongly sclerotized

34. Macropterous to brachypterous, if macropterous then with appendix large, extending around forewing apex; aedeagus movably hinged between base and shaft (Fig. 14H) (exceptions: Leofa, Picchusteles); ovipositor extending well beyond pygofer apex (exception: Paraphrodes); first valvula dorsal sculpturing pattern granulose to maculose, submarginal; second valvula teeth uniformly shaped, obtusely triangular and serrated; Cosmopolitan Chiasmini (part)

- Macropterous to brachypterous, if macropterous, with appendix not large or extending around forewing apex; aedeagus not movably hinged; ovipositor length variable; first valvula dorsal sculpturing variable; second valvula teeth not obtusely triangular and serrated

35. Crown narrow, width between eyes less or equal to median width of eye (Fig. 54A); crown completely shagreen to base; pronotum lateral margin not carinate; male pygofer declivous, directed posteroventrally, with few macrosetae, basolateral cleft absent or not membranous (Fig. 54D); ovipositor extending far beyond pygofer; first valvula with distinctly delimited ventroapical sculptured area; second valvula without teeth; Cosmopolitan Stenometopiini

- Crown not very narrow; discal portion of crown texture usually glabrous or striate (rarely shagreen in some Athysanini [e.g., Anoterostemma], some Pendarini [Chlorotettix and Copididonus], and Limotettigini); pronotum lateral margin usually carinate; male pygofer shape variable, basolateral cleft present, membranous; ovipositor not protruding far beyond pygofer apex; first valvula without distinctly delimited ventroapical sculpturing; second valvula with teeth

36. Dorsoventrally depressed or flattened leafhoppers (e.g., Fig. 24B); head strongly or sometimes weakly produced; crown broad, more or less parabolically shaped (Figs 24A, 28A); crown to face transition acutely angled; anterior margin sometimes foliaceous (Figs 24B, 28B); face lying slightly obliquely to horizontal plane (Figs 24B, 28B); lateral margin of pronotum long, nearly as long or longer than least width of eye (Figs 24A, 28A); first valvula dorsal sculpturing maculose to granulose, distinctly submarginal; second valvula without dorsal teeth(exception: Glossocratus) ...37

- Not particularly dorsoventrally depressed or flattened; head not strongly produced; crown broad or not, rarely parabolically shaped; crown to face transition less acute; face usually lying in a more upright plane; lateral margin of pronotum usually shorter than least width of eye; first valvula dorsal sculpturing variable; second valvulae with teeth .38

37. Ocelli close to eyes, closer to eyes than lateral frontal sutures (Fig. 28C); male apodemes of abdominal sternite I long, narrow, as long as apodemes of sternite II (Fig. 28J); male apodemes of abdominal sternite II large, spatulate (Fig. 28J); first and second valvulae humpbacked dorsally; second valvula concave ventrally; Cosmopolitan ...................................ecalini (part)

- Ocelli relatively distant from eyes, usually 3-4 x their diameter from ocellus or more (exception: some Faltala, Bonamus), sometimes absent or obscure; male apodemes of abdominal sternite I short or undeveloped; male apodemes of abdominal sternite II short or undeveloped; first and second valvulae not strongly humpbacked dorsally; second valvula not concave ventrally; Nearctic, Neotropical Faltalini (part) 
38. Body usually broad and robust (Fig. 13A-B); forewings brachypterous to submacropterous (Fig. 13A-B) (exception: Renosteria); hindwings when present narrowed anteriorly-posteriorly when outstretched or otherwise reduced in size (Fig. 13J-K) (exception: Renosteria); connective $\mathbf{U}$ - or sometimes $\mathbf{Y}$ - shaped with stem often very short or absent (Fig. 13G); male segment $\mathrm{X}$ broad and sclerotized; ovipositor usually distinctly protruding beyond pygofer apex (Fig. 13B); Afrotropical (South Africa) ........................................................... Bonaspeiini

- Body shape variable; forewings usually macropterous; hindwings not narrowed anteriorlyposteriorly when outstretched; connective variable; male segment $\mathrm{X}$ variable; ovipositor usually not protruding far beyond pygofer apex

39. Anterior margin of head with 2 or more distinct carinae or with several distinct transverse striations (e.g., Fig. 50C); antennal ledge with oblique carina (e.g., Fig. 49C); medium to large-sized leafhoppers

- Anterior margin of head without carinae or distinct transverse striations (rarely with single transverse carina), texture shagreen or glabrous, crown rounded to face; antennal ledge absent or represented by weak carina; size variable

40. Antenna long, at least as long as half length of body; antennal pits near upper (anterodorsal) corners of eyes, large and encroaching onto frontoclypeus (Fig. 20C) (exception: Nirvanguina); profemur row AV setae reduced or absent, usually with fine setae; Afrotropical, Oriental, Australian Drabescini (part, Paraboloponina)

- Antenna not very long; antennal pits near lower corners of eyes (exception: Selenocephalini [Dwightlina]), not very large or encroaching onto frontoclypeus); profemur row AV with distinct stout setae

41. Profemur row AM often with 1 or more extra macrosetae basad of AM1; pygofer usually with macrosetae reduced in number or absent (e.g., Fig. 52D) (exception: Adamina); connective stem usually very short or absent, connective U-shaped (Fig. 52G); male segment X usually broad and well-sclerotized dorsally; Afrotropical, Palearctic, Oriental ..........................Selenocephalini

- Profemur row AM usually without extra macrosetae basad of AM1; connective stem present, relatively long, connective $\mathbf{Y}$-shaped; male segment $\mathrm{X}$ variable, usually not very broad or large ......

42. Head distinctly narrower than pronotum (Fig. 46A); antennal ledges carinate (Fig. 46C); lorum long, relatively large (Fig. 46C); clypellus long, widening apically (Fig. 46C); pronotum lateral margin long, longer than half basal width of eye; forewing often with brown irrorate markings; profemur row AM sometimes with one or more setae basad of AM1; nymph with pygofer short, with 6 rows of abdominal macrosetae ...........................Phlepsiini (part)

- Head usually as wide as or wider than pronotum; antennal ledges variable; lorum not very large; clypellus usually not particularly long; pronotum lateral margin short, usually less than half basal width of eye; forewing coloration variable; profemur row AM only AM1 seta; nymph variable

43. Medium to large sized, robust leafhoppers (Fig. 12A-B); often predominantly brown in color (Fig. 12A-B); crown usually short, broad (Fig. 12A); often with anterior margin of head distinctly upturned, raised, or with several transverse carinae (Fig. 12B-C); clypellus widening apically, parallelmargined, or tapering apically (Fig. 12C), sometimes distinctly tumid (Fig. 12C); forewing often with strongly pigmented reflexed costal veins (Fig. 12B); pygofer often with processes (Fig. 12D); aedeagus often with apical processes (Fig. 12H-I); Nearctic, Neotropical ..... Bahitini (part)

- Without the above combination of characters; Cosmopolitan Athysanini (part) 
44. Aedeagus with single, unpaired sclerotized apophysis (= 'dorsal connective') projecting dorsally from atrium, membranously connected to segment X (Fig. 32H-I); male pygofer dorsal margin thickened, declivous, and often ending in ventrally directed curved hook or spine (Fig. 32D); Palearctic, Oriental, Australian, Nearctic, Neotropical ..............................................imotettigini

- Without the above combination of characters

45. Large, yellow to greenish species (Fig. 29A-B); forewing veins greenish (Fig. 29A-B); forewing venation highly reticulated apically (Fig. 29B); Afrotropical (South Africa) .........Hypacostemmini

- Color variable; forewing venation rarely highly reticulate, but if so, reticulate portion not restricted to forewing apex

46. Ocelli on face below anterior margin of head (Fig. 51C), distant from eyes; Afrotropical

Selenocephalini (part, Ianeirini: Ianeira)

- Ocelli on anterior margin of head, close to eyes

47. Head distinctly narrower than pronotum (Fig. 46A); antennal ledge carinate (Fig. 46C); lorum long, relatively large (Fig. 46C); clypellus long, widening apically (Fig. 46C); pronotum lateral margin longer than half basal width of eye (Fig. 46A); forewing often with brown irrorate markings (Fig. 46A-B); profemur row AM sometimes with one or more setae basad of AM1; nymph with pygofer short, with 6 rows of abdominal macrosetae ...........................Phlepsiini (part)

- Head width variable; antennal ledge variable; lorum not very large; clypellus usually not particularly long; pronotum lateral margin usually less than half basal width of eye; forewing coloration variable; profemur row AM without one or more macrosetae basad of AM1; nymph variable .......48

48. Medium to large sized, robust leafhoppers (12A-B); often predominantly brown in color (12A$\mathrm{B})$; crown usually short, broad (12A); often with anterior margin of head distinctly upturned, raised (12B-C); clypellus widening apically, parallel-margined, or tapering apically (12C), sometimes distinctly tumid (12C); forewing often with strongly pigmented reflexed costal veins (13B); pygofer often with processes (12D); aedeagus often with apical processes $(12 \mathrm{H}$ -

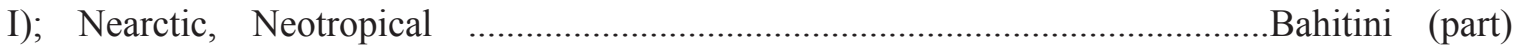

- Size variable; color variable; anterior margin of head usually not upturned, raised; clypellus usually widening apically or parallel-sided, not tumid; forewing with or without reflexed costal veins; pygofer with or without processes; aedeagus with or without apical processes

49. (Note: the following characters are not present in all included genera, but some combination of most of these characters is present in all): Head narrower than pronotum, produced anteriorly (Fig. 47A); frontoclypeus long, narrow (Fig. 47C); antennae long, longer than width of head; body slender (Fig. 47A); head and wings often with brown, orange, ochraceous, or ivory markings (Fig. 47A-B); forewing with one or more darkly pigmented reflexed costal veins (Fig. 47B); profemur row AV setae absent or reduced (not stout); metatibia row PD macrosetae long, as long as or longer than $0.5 x$ length of protibia; male or female pygofer with dense tufts of long fine or regular setae (Fig. 47D); subgenital plate apex membranous or long, digitate, somewhat membranous or weakly sclerotized; subgenital plates with long fine setae laterally (Fig. 47F); basal processes of aedeagus or connective often present, connected or articulated to base of aedeagus or apex of connective stem (Fig. 47J, M); Cosmopolitan ................Scaphoideini

- Without some combination of above characters .50

50. Ground color yellowish or greenish OR forewing with numerous brown, tawny, or reddishbrown ramose or reticulate pigment lines, or connected dots forming such lines (Fig. 44A-B); head as wide as or wider than pronotum; male pygofer often with a spine or process arising 
from dorsal side; nymph crown-face transition carinate; face of nymph with longitudinal carina in upper part; nymph pygofer elongate; Nearctic, Neotropical Pendarini

- Without the above combination of characters; Cosmopolitan Athysanini (part)

\section{Acinopterini Oman, 1943}

Fig. 8

Type genus: Acinopterus Van Duzee, 1892.

\section{Diagnosis}

Acinopterini are medium sized, yellowish, greenish, orange, or brown leafhoppers. They can be identified by the straight commissural margin of the forewing, forewing apices not overlapping, forewing appendix absent or reduced, connective bar-shaped or plate-like, style slender (not broadly bilobed), and female first valvula strongly curved.

\section{Description}

HEAD. Head distinctly narrower than pronotum (Acinopterus) or subequal to or wider than pronotum (Cariancha). Discal portion of crown glabrous with radial or longitudinal striae. Anterior margin of head shagreen. Frontoclypeus not tumid; texture shagreen. Clypellus widening apically. Clypellus apex following or slightly surpassing normal curve of gena. Lorum subequal to or wider than clypellus near base. Antennal bases near middle or posteroventral (lower) corners of eyes. Antennae short, less than $1.5 \mathrm{x}$ width of head. Gena obtusely incised laterally; with fine erect seta beside laterofrontal suture. Antennal ledge weakly developed (carinate or weakly carinate). Ocelli present; close to eyes; on anterior margin of head.

Thorax. Pronotum lateral margin carinate; lateral margin shorter than basal width of eye.

WINGS. Forewing macropterous; with commissural margin straight; apex acuminate; appendix absent or reduced; with 3 anteapical cells; veins not raised; without reflexed costal veins; A1-A2 crossvein absent.

Legs. Profemur with AM1 seta only or with one or more additional proximal setae; intercalary row with one row of five or more fine setae; row AV with relatively long macrosetae. Protibia dorsal surface rounded, convex. Metafemur apex macrosetae $2+2+1$. Metatarsomere I not expanded apically; plantar setae simple, tapered.

Male genitalia. Valve articulated with pygofer; lateral margin short, articulating with pygofer at a point. Pygofer basolateral membranous cleft present; macrosetae well differentiated into several rows. Subgenital plates free from each other; articulated with valve; without macrosetae. Style linear, median anterior lobe not pronounced. Basal processes of the aedeagus present, fused to base of aedeagus. Aedeagus with single shaft and gonopore. Connective anterior arms widely divergent; bar-shaped or plate-like; articulated with aedeagus.

Female genitalia. Pygofer with numerous macrosetae. Ovipositor not protruding far beyond pygofer apex. First valvula convex; dorsal sculpturing pattern strigate, concatenate, reticulate, or imbricate (with overlapping scales); sculpturing reaching dorsal margin; without distinctly delimited ventroapical sculpturing. Second valvula abruptly broadened medially or subapically or broad, gradually tapered apically; without dorsal median tooth; teeth on apical $1 / 3$ or more; teeth small, regularly or irregularly shaped. 


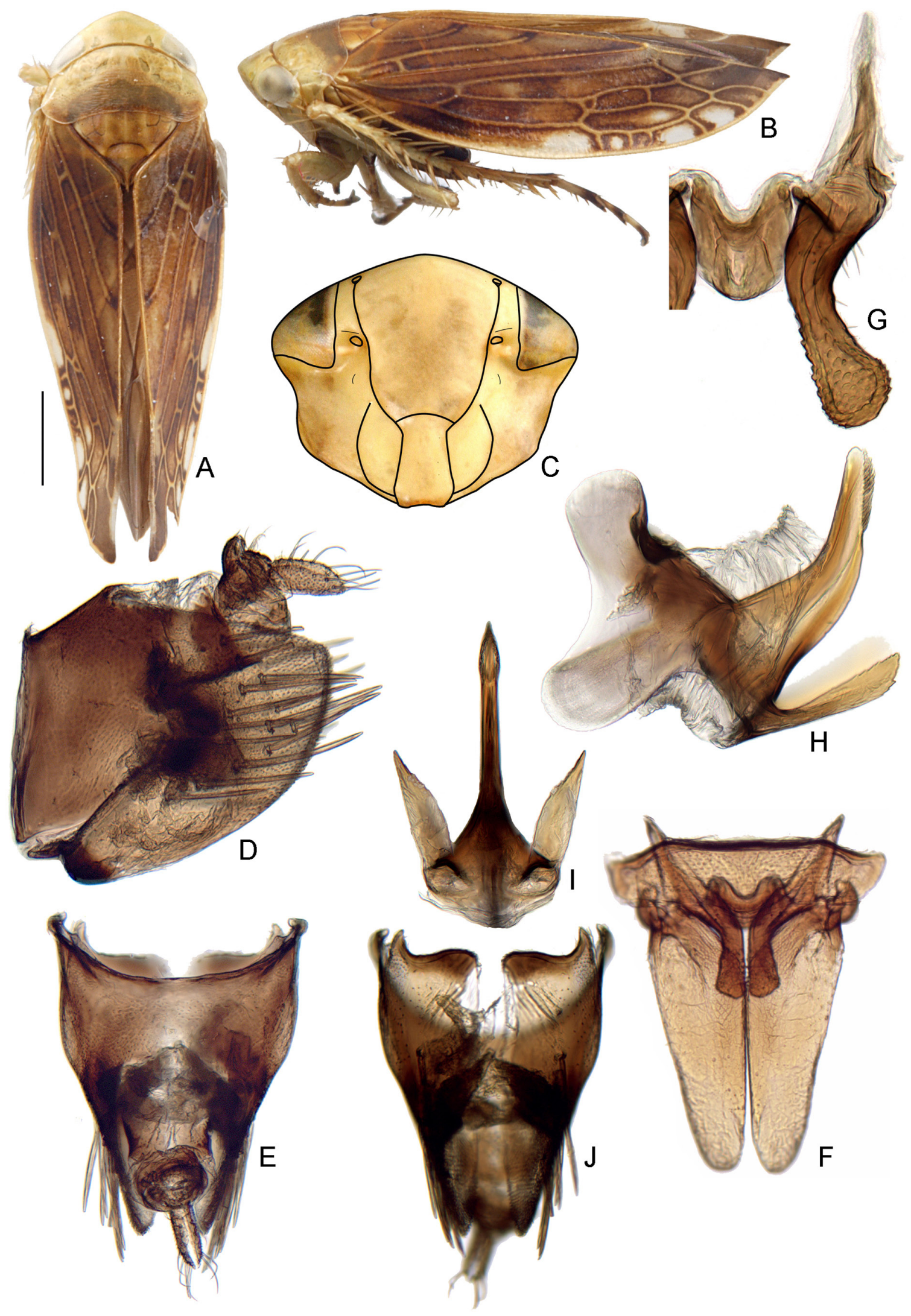

Fig. 8. Acinopterini. Acinopterus acuminatus Van Duzee, 1892. A-I. Standard views (see Material \& Methods). J. §o pygofer, ventral view. 


\section{Geography and ecology}

Distribution: Nearctic, Neotropical. Acinopterus species live in meadows, grasslands, and in forest understories and feed on a variety of herbaceous dicots. Acinopterus angulatus Lawson, 1922 is a vector of the western strain of aster yellows and western X disease.

\section{Remarks}

Acinopterini contains 2 genera and 30 species. Acinopterus (28 spp.) is widely distributed from North to South America and Cariancha (2 spp.) is known only from Brazil. This is an early-diverging tribe of Deltocephalinae and it possesses plesiomorphic characters of the male genitalia. Among the other early-diverging lineages of Deltocephalinae, the $28 \mathrm{~S}$ rDNA sequence of Acinopterus was updated to include sequences only from $A$. acuminatus Van Duzee, 1892 (previously a chimeric sequence of $A$. acuminatus and $A$. viridis Ball, 1903 was used) and the D2 region of Luheria was included for the first time. However, these changes did little to resolve the relationships between Acinopterini and the other early-diverging deltocephaline tribes, Stegelytrini, Acostemmini, Luheriini and Fieberiellini. Future analyses should include more representatives of these tribes and representatives of Cariancha to facilitate further understanding of their relationships.

\section{Selected references}

Beamer \& Lawson (1938), Oman (1949), Linnavuori \& DeLong (1977), Cwikla \& Ma (1986).

\section{Included Genera}

Acinopterus Van Duzee, 1892.

Cariancha Oman, 1938.

Acostemmini Evans, 1972

Fig. 9

Type genus: Acostemma Signoret, 1860.

= Acroponinae Linnavuori \& Quartau, 1975.

\section{Diagnosis}

Acostemmini are medium sized to very large yellow, greenish, reddish-brown, brown, or greyish leafhoppers. They can be identified by the single carina on the anterior margin of the head, distinct epistomal suture, forewing with a straight commissural margin (apices not overlapping) and without appendix, pygofer setae reduced or absent, valve fused to pygofer or lateral margin long and articulated with pygofer, style linear-shaped, and subgenital plates sometimes fused to each other.

\section{Description}

HEAD. Head subequal to or wider than pronotum. Discal portion of crown glabrous with radial or longitudinal striae. Anterior margin of head with a single transverse carina. Frontoclypeus not tumid; texture mostly shagreen; epistomal suture distinct or evident by change in texture from glabrous, strigate, or irregular above suture to shagreen below. Clypellus widening apically; sometimes long and hourglassshaped. Clypellus apex following or slightly surpassing normal curve of gena. Lorum subequal to or wider than clypellus near base. Antennal bases near middle or posteroventral (lower) corners of eyes. Antennae short, less than $1.5 \mathrm{x}$ width of head. Gena obtusely incised laterally; with fine erect seta beside laterofrontal suture. Antennal ledge weakly developed (carinate or weakly carinate). Ocelli present; close to eyes; on anterior margin of head. 


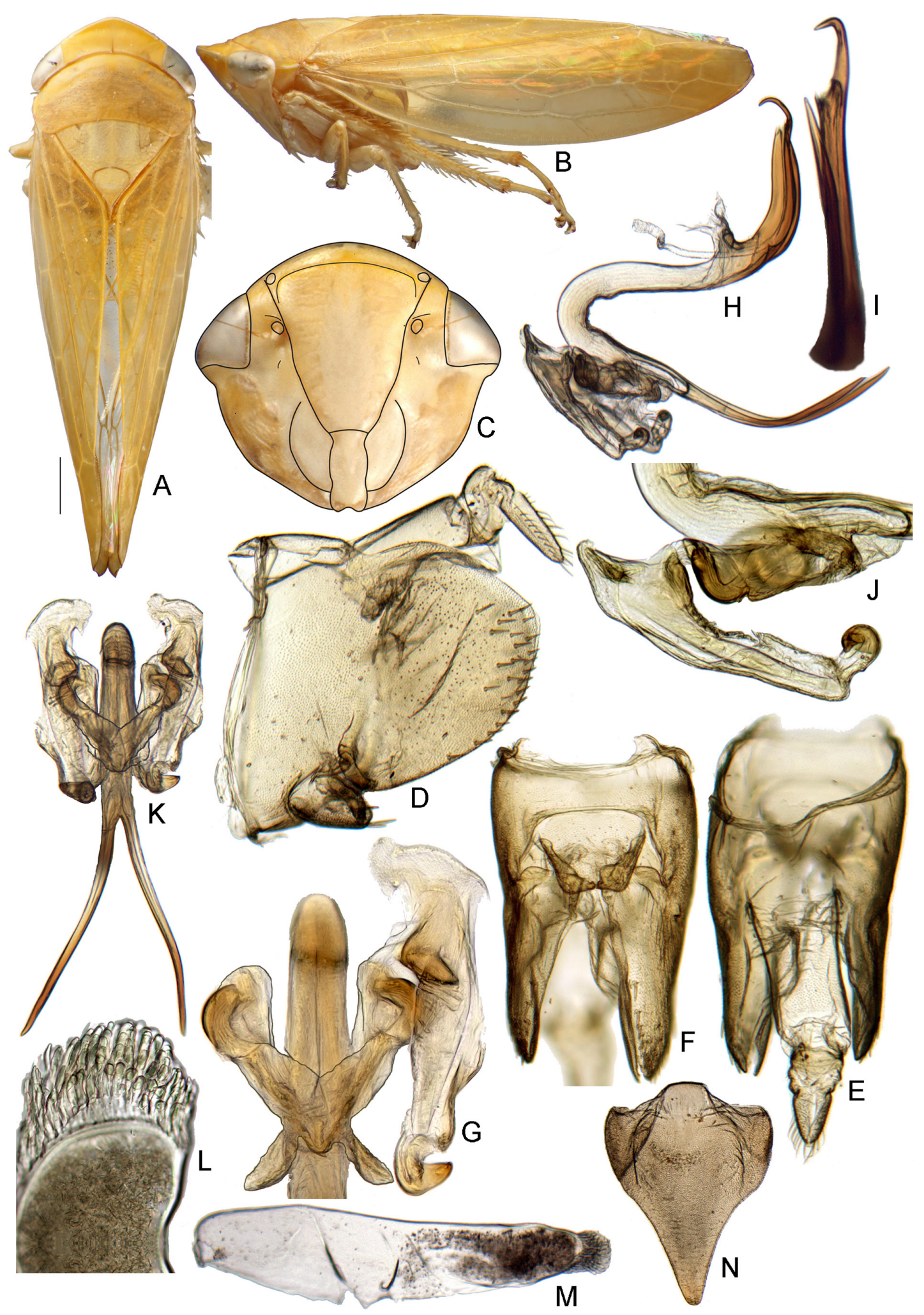

Fig. 9. Acostemmini. Acostemma stilleri sp. nov. A-I. Standard views (see Material \& Methods). F. Plates shown with pygofer. G. Connective and style shown with part of fused aedeagus. H. Aedeagus shown with fused processes, connective, and style. J. Lateral view of style, connective, and base of aedeagus. K. Ventral view of connective, styles, and ventral processes of aedeagus. L. Detail of hairlike extensions of egg. M. Egg. N. + sternite VII. 
Thorax. Pronotum lateral margin carinate; lateral margin shorter than basal width of eye.

WINGS. Forewing macropterous, submacropterous, or rarely subbrachypterous; appendix absent or reduced; with 3 anteapical cells; veins not raised; veins sometimes with callosities; without reflexed costal veins; A1-A2 crossvein present; apical venation strongly reticulate or not reticulate.

LEGs. Profemur with AM1 seta only or with one or more additional proximal setae; intercalary row with one row of five or more fine setae; row AV with short, stout setae or with relatively long macrosetae. Protibia dorsal surface rounded and convex or flat (AD and PD margins at $\sim 90^{\circ}$ angles but not carinate). Metafemur apex macrosetae with $2+2+1$. Metatarsomere I not expanded apically; plantar setae simple, tapered.

MaLe Genitalia. Valve straplike, sometimes longer laterally than medially (Acostemella); articulated with or fused to pygofer; if articulated then lateral margin long, with length of articulation long; articulated with or fused to subgenital plates. Pygofer basolateral membranous cleft absent; macrosetae reduced or absent or well differentiated into several rows. Subgenital plates fused or articulated with each other; articulated with or fused to valve; without macrosetae, with macrosetae irregularly arranged, or with macrosetae uniseriate laterally. Style linear, median anterior lobe not pronounced or broadly bilobed basally, median anterior lobe pronounced; apophysis sometimes bent at nearly $90^{\circ}$ angle. Basal processes of the aedeagus/connective absent or present, if present then connected to or articulated with connective or base of aedeagus. Aedeagus with single shaft and gonopore. Connective anterior arms widely divergent, T-shaped, or somewhat divergent, Y-shaped; sometimes with an anteromedial lobe or process (Acostemella, Alocoelidia, Caelidioides, Ikelibeloha, Iturnoria); fused to or articulated with aedeagus.

Female genitalia. Pygofer with numerous macrosetae. Ovipositor not protruding far beyond pygofer apex. First valvula convex or not strongly convex; dorsal sculpturing pattern strigate, concatenate, or reticulate; sculpturing reaching dorsal margin; without distinctly delimited ventroapical sculpturing. Second valvula broad, gradually tapered or slender throughout, without dorsal median tooth; teeth restricted to apical $1 / 4$ or less; teeth small, regularly or irregularly shaped.

\section{Geography and ecology}

Distribution: Afrotropical and Oriental (India, Sri Lanka, New Guinea) regions. The diversity of the tribe lays mostly in Madagascar, where it appears to have radiated and subsequently dispersed to mainland Africa, India, and Sri Lanka where species of Acostemma are found. Several undescribed genera and many species have recently been discovered (Zahniser \& Nielson 2012) based on samples from an arthropod biodiversity inventory of Madagascar. Some species in the genus Eryapus resemble bird droppings.

\section{Remarks}

Acostemmini contains 12 genera and 27 species. This subfamily is a relictual lineage of Deltocephalinae and retains some primitive features of the male genitalia.

The morphologically enigmatic genus Ikelibeloha and four other genera were included in the tribe by Zahniser \& Nielson (2012). They noted that these genera do not share all of the male genitalic characters that previously helped to define the tribe (e.g., valve fused to pygofer) but shared other unique acostemmine characters that supported their placement in the tribe. Ikelibeloha and Iturnoria were included in phylogenetic analyses using only molecular data by Zahniser \& Nielson (2012) and formed a monophyletic group with the other sampled acostemmines (Acostemma and Eryapus). In the MP analysis here, Ikelibeloha and Iturnoria did not group with the other acostemmines, a result which is 
likely due to the inclusion of morphological data and the divergent morphology of these taxa as compared to "typical" Acostemmini. However the ML and Bayesian analyses of combined data recovered the two genera as sister to the other included Acostemmini, Acostemma and Eryapus, with strong branch support (85 BS support and 1.0 PP, respectively), thus forming a monophyletic Acostemmini. The previous MP, ML, and Bayesian analyses of only molecular data also strongly supported this relationship (Zahniser \& Nielson 2012). Despite the result of the MP analysis here, the relationships among the earliest lineages of Deltocephalinae as those depicted in the ML and Bayesian trees here (both with moderate to high branch support) showing Stegelytrini as the earliest diverging tribe sister to a monophyletic Acostemmini and the remaining Deltocephalinae are the most well-supported by the available evidence.

Evans (1972b) tentatively placed Telopetulcus (not examined in this study), recorded only from New Guinea, in Acostemmini. Its placement is still uncertain. Fig. 6A of Evans (1972b) suggests the presence of the epistomal suture on the face which would indicate that it belongs in Acostemmini, but some other features (e.g., Y-shaped connective articulated with aedeagus) do not occur in other genera of the tribe. Closer examination of this genus is needed with reconsideration of its tribal placement.

\section{Selected references}

Evans (1954, 1972b), Linnavuori \& Quartau (1975), Linnavuori \& Al-Ne'amy (1983), Zahniser \& Nielson (2012).

\section{Included genera}

Acostemana Evans, 1954

Acostemma Signoret, 1860

Acostemmella Evans, 1954

Alocoelidia Evans, 1954

Caelidioides Signoret, 1880

Dardania Stål, 1866

Eryapus Evans, 1954

Ikelibeloha Zahniser \& Nielson, 2012

Iturnoria Evans, 1954

Malicia Evans, 1954

Protonesis Spinola, 1850

Telopetulcus Evans, 1972

Arrugadini Linnavuori, 1965

Fig. 10

Type genus: Arrugada Oman, 1938.

\section{Diagnosis}

Arrugadini are large brown and yellow leafhoppers. They can be easily identified by the strongly rugose texture of the head and face, lateral margins of the pronotum longer than the basal width of the eye, first valvula dorsal sculpturing pattern granulose and submarginal, first valvula with distinctly delimited ventroapical sculpturing, and second valvula gradually expanded apically and without teeth.

\section{Description}

HEAD. Head subequal to or wider than pronotum. Discal portion of crown rugose or longitudinally striate. Anterior margin of head irregularly textured, rugose. Frontoclypeus tumid or not tumid; texture rugose. Clypellus widening apically. Clypellus apex following or slightly surpassing normal curve of 


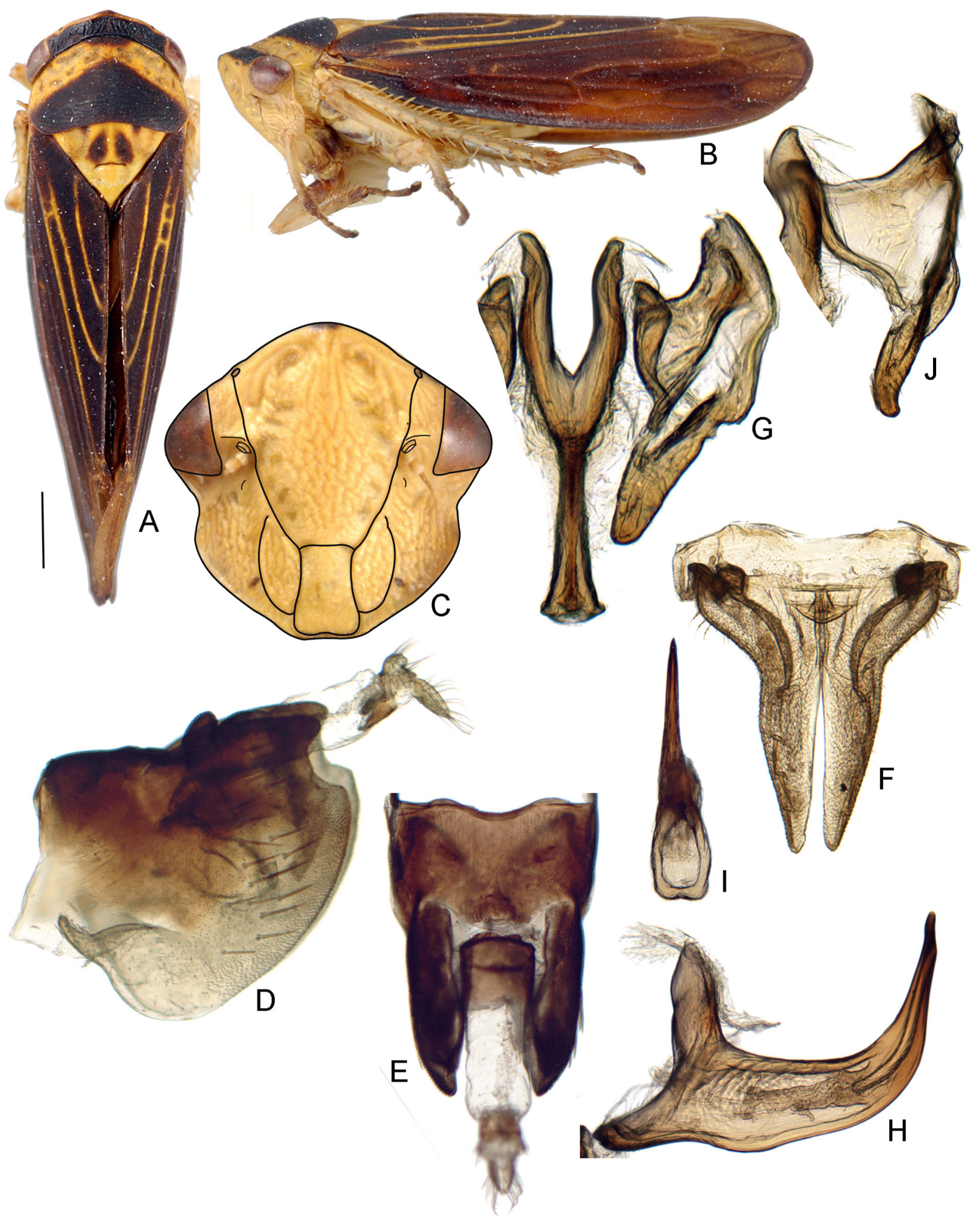

Fig. 10. Arrugadini. A-B, D-J. Arrugada linnavuorii sp. nov. C. Arrugada sp. A-I. Standard views (see Material \& Methods). J. Broad view of style. 
gena. Lorum subequal to or wider than clypellus near base. Antennal bases near middle or posteroventral (lower) corners of eyes. Antennae short, less than $1.5 \mathrm{x}$ width of head. Gena obtusely incised laterally; with fine erect seta beside laterofrontal suture. Antennal ledge weakly developed (carinate or weakly carinate). Ocelli present; close to or somewhat distant from eyes; on anterior margin of head.

Thorax. Pronotum not exceeding or exceeding (female of $A$. rugosa) eyes anteriorly; lateral margin carinate; lateral margin as long as or longer than basal width of eye.

WINGS. Forewing macropterous or submacropterous; appendix restricted to anal margin; with 3 anteapical cells; veins distinctly raised or carinate; without reflexed costal veins; A1-A2 crossvein absent.

Legs. Profemur with AM1 seta only; intercalary row with one row of five or more fine setae; row AV with relatively long macrosetae. Protibia dorsal surface flat, $\mathrm{AD}$ and $\mathrm{PD}$ margins at $\sim 90^{\circ}$ angles but not carinate. Metafemur apex macrosetae $2+2+1$ or $2+1+1$. Metatarsomere I not expanded apically; plantar setae simple, tapered.

Male Genitalia. Sternal apodemes of segments I and II small, not well developed. Valve articulated with pygofer; lateral margin short, articulating with pygofer at a point. Pygofer basolateral membranous cleft present; macrosetae well differentiated into several rows. Subgenital plates free from each other; articulated with valve; macrosetae uniseriate laterally. Style broadly bilobed basally, median anterior lobe pronounced. Basal processes of the aedeagus/connective absent or reduced. Aedeagus with single shaft and gonopore. Connective anterior arms somewhat divergent, Y-shaped; articulated with aedeagus.

Female genitalia. Pygofer macrosetae reduced or absent. First valvula not strongly convex; dorsal sculpturing pattern granulose or maculose; sculpturing submarginal; with ventroapical sculpturing distinctly delimited. Second valvula gradually broadened medially or subapically; without dorsal median tooth; teeth absent.

\section{Geography and ecology}

Distribution: Neotropical. Host plants are not known. Large numbers of males of Arrugada affinis (Osborn, 1924) were observed and collected while "mud-puddling" in Peru (Rakitov et al. 2005).

\section{Remarks}

Arrugadini contains only the type genus and 4 valid species from Bolivia and Peru. This small group was considered a separate subfamily until it was recently included as a tribe of Deltocephalinae (Zahniser \& Dietrich 2010). Based on some characters of the female genitalia (first valvula dorsal sculpturing pattern granulose and submarginal, first valvula with distinctly delimited ventroapical sculpturing, second valvula without dorsal teeth), it appears to be related to the grass-feeding tribe Hecalini, a relationship that was marginally supported in the combined parsimony analyses presented here. However, the shapes of the first and second valvulae are not very similar to Hecalus and Attenuipyga which have humpbackshaped valvulae. The relationships within Hecalini and between this and other tribes seem particularly difficult to recover given the data in this study. More data are needed to resolve the relationships between Arrugadini and other Deltocephalinae. Preliminary phylogenetic analyses of Hecalini (Catanach, unpublished) suggest that Arrugada is related to another South American-endemic genus, Egenus. Egenus was removed from Hecalini by Hamilton (2000), tentatively placed in Faltalini by Zahniser \& Dietrich (2010), and is transferred to Athysanini here (see below).

\section{Selected references}

Linnavuori \& DeLong (1978a), Zahniser \& Dietrich (2010). 


\section{Included genera}

Arrugada Oman, 1938.

Athysanini Van Duzee, 1892

Fig. 11

Type genus: Athysanus Burmeister, 1838.

$=$ Phrynomorphini Kirkaldy, 1907.

$=$ Thamnotettigini Distant, 1908.

= Euscelini Van Duzee, 1917.

$=$ Anoterostemmini Haupt, 1929 syn. nov.

= Bobacellini Kusnezov, 1929.

= Platymetopiini Haupt, 1929 syn. nov.

= Colladonini Bliven, 1955.

$=$ Cerrillini Linnavuori, 1975.

= Allygidiina Dmitriev, 2006 syn. nov.

\section{Diagnosis}

Because this is such a large tribe, it is difficult or impossible to provide a set of characters that will easily diagnose it. There is substantial morphological diversity in the group, but most members have a Y-shaped connective and lack the distinctive features of other tribes.

\section{Description}

HEAD. Head subequal to or wider than pronotum or distinctly narrower than pronotum. Discal portion of crown glabrous, radially or longitudinally striate, or shagreen. Anterior margin of head shagreen, glabrous, irregularly textured, with numerous transverse striations, foliaceous, with 2 or 3 parallel carinae, or with numerous carinae. Frontoclypeus not tumid or tumid; texture shagreen or rugose. Clypellus parallel-sided or widening apically; apex following or slightly surpassing normal curve of gena. Lorum subequal to or wider than clypellus near base or distinctly narrower than clypellus near base. Antennal bases near middle or posteroventral (lower) corners of eyes. Antennae short, less than $1.5 \mathrm{x}$ width of head or long, $1.5 \mathrm{x}$ width of head or longer. Gena obtusely incised laterally; with fine erect seta beside laterofrontal suture. Antennal ledge absent or weakly developed (carinate or weakly carinate). Ocelli present; close to eyes or distant from eyes; on anterior margin of head.

THORAX. Pronotum lateral margin carinate or not; lateral margin shorter than basal width of eye.

WiNGS. Forewing brachypterous to macropterous; appendix absent or reduced (in submacropterous or brachypterous forms) or restricted to anal margin; with 3 anteapical cells; veins not raised; with or without reflexed costal veins; A1-A2 crossvein absent or present; apical venation usually not strongly reticulate, rarely reticulate.

LeGs. Profemur with AM1 absent or reduced, with AM1 seta only, or with AM1 and with one or more additional proximal setae; intercalary row with one row of five or more fine setae; row AV with thin, hair-like setae, without setae, with short, stout setae, or with relatively long macrosetae. Protibia dorsal surface rounded, convex. Metafemur apex macrosetae $2+2+1$ or $2+2+1$ with additional proximal macrosetae. Metatarsomere I not expanded apically or expanded apically; plantar setae usually simple, tapered. 


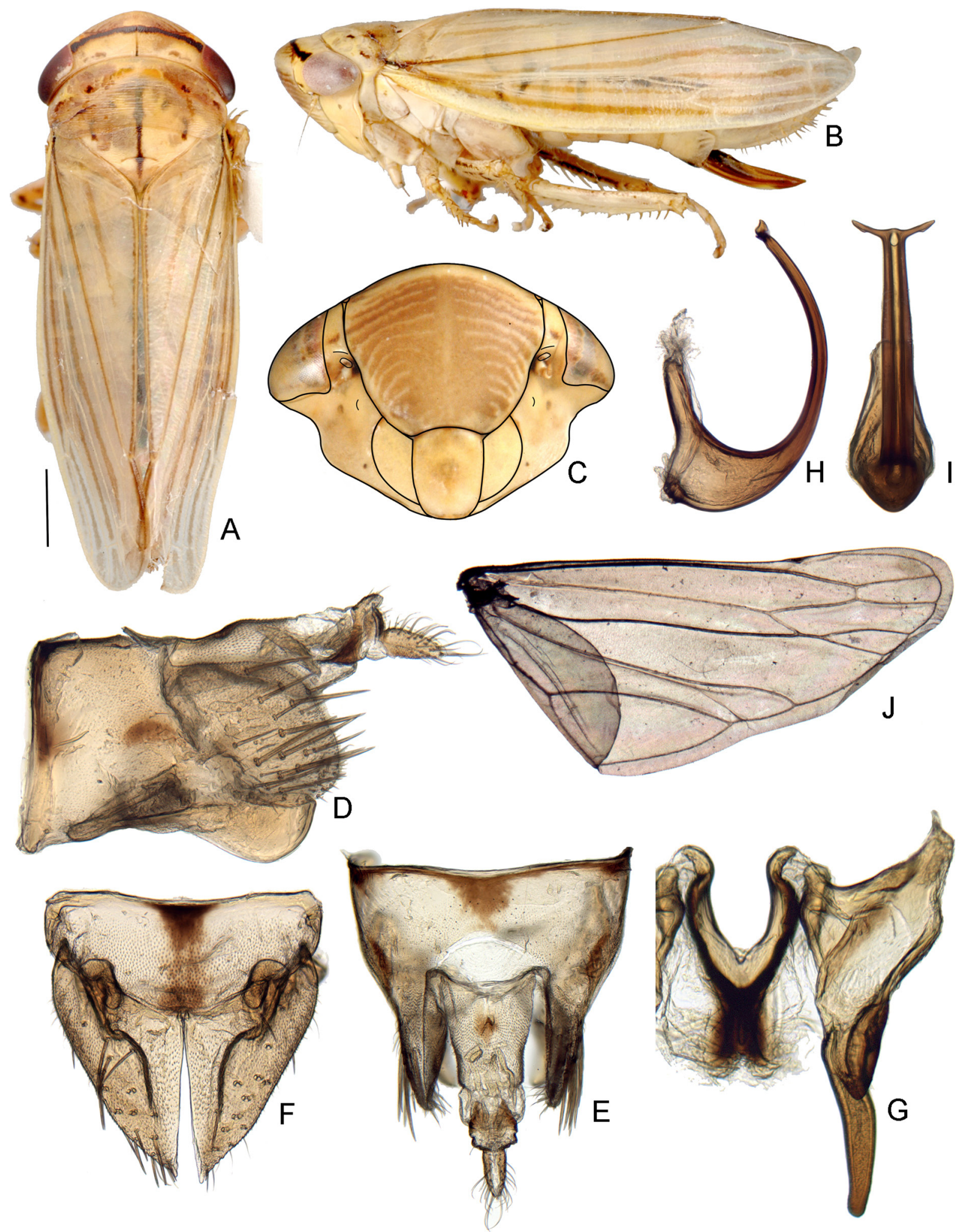

Fig. 11. Athysanini. Athysanus argentarius Metcalf, 1955. A-I. Standard views (see Material \& Methods). J. Hindwing. 
Male genitalia. Valve articulated with pygofer or fused to subgenital plates; lateral margin short, articulating with pygofer at a point. Pygofer basolateral membranous cleft present; macrosetae absent or reduced ( $\leq$ two rows) or macrosetae well differentiated into several rows. Subgenital plates free from each other; articulated with or rarely fused to valve; macrosetae absent or present, scattered and irregularly arranged, uniseriate laterally, with two lateral rows, with some irregularly arranged macrosetae near lateral margin, or uniseriate and distant from lateral margin. Style broadly bilobed basally, median anterior lobe pronounced. Basal processes of the aedeagus/connective absent or reduced or present, connected or articulated to connective or near base of aedeagus. Aedeagus with single shaft and gonopore. Connective anterior arms somewhat divergent, Y- or $\mathbf{U}$-shaped, or rarely anterior arms closely appressed anteriorly; articulated with or rarely fused to aedeagus.

Female genitalia. Pygofer with numerous macrosetae. Ovipositor not protruding far beyond or protruding far beyond pygofer apex. First valvula convex or not strongly convex; dorsal sculpturing pattern strigate, concatenate, reticulate, or imbricate (with overlapping scales); sculpturing reaching dorsal margin or submarginal; with indistinctly delimited ventroapical sculpturing or rarely with ventroapical sculpturing distinctly delimited. Second valvula abruptly broadened medially or subapically or broad, gradually tapered; with or without dorsal median tooth; dorsal teeth on apical 1/3 or more; teeth large, regularly shaped or small, regularly or irregularly shaped.

\section{Geography and ecology}

Distribution: cosmopolitan. Athysanini can be found in nearly all terrestrial ecosystems. Athysanini feed on a wide variety of eudicots and some species occasionally feed on grasses or sedges. This group contains numerous vectors of crop diseases. Some of the most well-known include: Colladonus clitellarius (Say, 1830), C. geminatus (Van Duzee, 1890) and C. montanus (Van Duzee, 1892), which transmit Eastern and Western X-diseases of peach, aster yellows, yellow leaf roll stain, and Euscelis incisus (Kirschbaum, 1858), a vector of numerous diseases of clover.

\section{Remarks}

This is the largest tribe of Deltocephalinae, including 228 genera and 1123 species. Habitus images are available for most genera of the tribe through the online database. Athysanini constitute a polyphyletic assemblage of genera which have historically been placed in the tribe mostly because they retain the most common deltocephaline external and genitalic characters but lack the distinctive features that define other tribes. More detailed studies of the phylogeny of Deltocephalinae including many more representatives of Athysanini are needed to more fully understand the relationships of these genera and the evolution of all of Deltocephalinae. Such studies may also help to determine morphological characters that define smaller, more particular lineages, particularly those belonging to clades that received strong branch support in our phylogenetic analyses. For now, the revised classification presented here including the new tribes Bahitini and Phlepsiini and the revised interpretation of Scaphoideini separates some groups of genera which were previously placed in Athysanini or were previously unplaced to tribe. Revisions to Athysanini here include the addition of many genera that were previously unplaced in Deltocephalinae, thus resulting in a classification of the subfamily that has no genera unplaced to tribe. This arrangement seems preferable to an alternative option of restricting Athysanini to the interpretation of the Athysanus genus group, as defined here, and considering all other genera once included in Athysanini to be unplaced to tribe in Deltocephalinae. The latter option would likely create more confusion, as did the previous classification which contained both unplaced genera and a large number of genera in Athysanini without any clear distinction between the two.

Other changes made here include considering Allygidiina, Anoterostemmina, and Platymetopiina synonyms of Athysanini. Emeljanov (1999) and Dmitriev (2006b) recognized up to 4 subtribes of Athysanini (Allygidiina, Athysanina, Cicadulina, and Platymetopiina) although their generic 
classifications partly conflict. Both authors included the genera treated here as Cicadulini in Athysanini (Emeljanov in Athysanina, and Dmitriev as the subtribe Cicadulina). The phylogenetic analyses here included representatives of 5 of the 14 genera included in Platymetopiina by Dmitriev (2006b): Anoplotettix, Colladonus, Phlepsius, Platymetopius, and Thamnotettix. Except for a relationship between Colladonus and Platymetopius, the analyses strongly suggest that these genera are not closely related and that the morphological characters previously used to define the group are not informative of phylogenetic relationships. Therefore, Platymetopiina is not recognized here. Further investigation may allow for a revised concept of this group. The genera Eusama and Twiningia were found with strong branch support to be related to Colladonus and Platymetopius, which could in part inform future recognition of such a taxon. The phylogenetic analyses grouped the included representatives of Athysanina and Allygidiina in the same area of the tree and they share some morphological features. They are therefore recognized together in the informal Athysanus genus group, and some other genera that were not within the geographical ranges of the previous taxonomic studies are included in the group.

The morphologically unique Neotropical genus Chimaerotettix was included in our analyses, but its placement with respect to other Athysanini was poorly resolved.

Justification for the inclusion of other genera not covered in the previous discussion is given below.

Egenus was previously included in Faltalini by Zahniser \& Dietrich (2010) who had not examined female specimens. It is transferred to Athysanini here because female specimens became available for study which showed that they do not possess the synapomorphies of the first and second valvulae that in part define Faltalini. Preliminary phylogenetic work on the tribe Hecalini (Catanach, personal communication) suggests that Egenus may be related to Arrugada.

Loralia is transferred to Athysanini from Deltocephalini. It clearly does not belong in Deltocephalini and was found here to be closely related to Occinirvanini. However, we were unable to identify morphological features uniting Loralia and Occinirvana, so more study of the Australian fauna will be needed to determine whether including Loralia or other genera in Occinirvanini is justifiable.

Nesothamnus is transferred from Scaphytopiini. It is not closely related to Scaphytopius but is potentially more closely related to some Neotropical Athysanini. In several analyses, it was resolved as sister to or near Idioceromimus with which it shares a relatively short, broad body form and bright orange/red coloration.

Phycotettix was listed in Fieberiellini by Oman et al. (1990). It does not possess the characters that are diagnostic for Fieberiellini and its placement there was perhaps a mistake due to the similarity of the name to the fieberielline genus Placotettix. Phycotettix was not included in Fieberiellini in a comprehensive revision of the tribe (Meyer-Arndt \& Remane 1992a, b).

\section{Selected references}

Oman (1949), Linnavuori (1959), Linnavuori \& DeLong (1978b), Cwikla \& Blocker (1981), Anufriev \& Emeljanov (1988), Emeljanov (1999), Dmitriev (2006b).

\section{Included genera}

Abrus Dai \& Zhang, 2002

Acacimenus Dlabola, 1979

Acunasus DeLong, 1945 placement nov. (previously unplaced in Deltocephalinae)

Aeternus Distant, 1918 placement nov. (previously unplaced in Deltocephalinae)

Aindrahamia Linnavuori, 1965

Aligia Ball, 1907 
ZAHNISER J.N. \& DIETRICH C.H., A review of the tribes of Deltocephalinae (Cicadellidae)

Alladanus DeLong \& Harlan, 1968

Allophleps Bergroth, 1920

Allotapes Emeljanov, 1964 placement nov. (previously placed in Platymetopiina)

Amblytelinus Lindberg, 1954

Anaemotettix Korolevskaya, 1980 placement nov. (previously placed in Platymetopiina)

Ancudana DeLong \& Martinson, 1974

Andanus Linnavuori, 1959

Angubahita DeLong, 1982

Angulanus DeLong, 1946

Antoniellus Linnavuori, 1959

Aplanatus Cheng, 1980

Aplanus Oman, 1949

Aplanusiella Nielson \& Haws, 1992

Arahura Knight, 1975

Arawa Knight, 1975

Argyrilla Emeljanov, 1972

Aricanus Linnavuori, 1959

Artucephalus DeLong, 1943

Asiotoxum Emeljanov, 1964

Asthenotettix Korolevskaya, 1980 placement nov. (previously placed in Platymetopiina)

Atanus Oman, 1938

Awasha Heller \& Linnavuori, 1968

Bambusana Anufriev, 1969

Bambusananus Li \& Xing, 2011

Bandara Ball, 1931

Bardana DeLong, 1980 placement nov. (previously unplaced in Deltocephalinae)

Baroma Oman, 1938

Benibahita Linnavuori, 1959

Bergolix Linnavuori, 1959

Bicoloratum Dai \& Li, 2011

Biluscelis Dlabola, 1980

Bolotheta Kramer, 1963

Bonneyana Oman, 1949

Brachylope Emeljanov, 1962

Brachypterona Lindberg, 1954

Branchana Li, 2011

Brasilanus Linnavuori, 1959

Brazosa Oman, 1938

Cahya Linnavuori, 1959

Caladonus Oman, 1949

Caranavia Linnavuori, 1959

Carelmapu Linnavuori, 1959

Cenedaeus Distant, 1908

Cerrillus Oman, 1938

Cetexa Oman, 1949

Chaparea Linnavuori, 1959

Chimaerotettix Dietrich \& Rakitov, 2002 placement nov. (previously unplaced in Deltocephalinae)

Chroocacus Emeljanov, 1962

Cocrassana Blocker \& Larsen, 1991

Colladonus Ball, 1936 placement nov. (previously placed in Platymetopiina) 
Comayagua Linnavuori \& DeLong, 1978

Conoscelis Abdul-Nour, 1988

Consepusa Linnavuori \& DeLong, 1977

Conversana DeLong, 1967

Costamia DeLong, 1946

Cozadanus DeLong \& Harlan, 1968

Crassana DeLong \& Hershberger, 1947

Cyanidius Emeljanov, 1964 placement nov. (previously placed in Platymetopiina)

Cyclevertex Li \& Xing, 2011

Dagama Distant, 1910

Dampfiana DeLong \& Hershberger, 1948

Danbara Oman, 1949 placement nov. (previously unplaced in Deltocephalinae)

Deltorhynchus DeLong, 1943

Desertana DeLong \& Martinson, 1973

Devolana DeLong, 1967

Didymotettix Yang, 1996

Divitiacus Distant, 1918

Dlabolaracus Remane, 1961

Doleranus Ball, 1936

Dolyobius Linnavuori, 1959

Egenus Oman, 1938 placement nov. (transferred from Faltalini)

Elphnesopius Nast, 1984

Ephelodes Emeljanov, 1972 placement nov. (previously placed in Platymetopiina)

Euleimonios Kirkaldy, 1906 placement nov. (previously unplaced in Deltocephalinae)

Eusama Oman, 1949 placement nov. (previously placed in Platymetopiina)

Eusceloidia Osborn, 1923 placement nov. (previously unplaced in Deltocephalinae)

Eusora Oman, 1949

Eutettix Van Duzee, 1892

Excavanus DeLong, 1946

Fistulatus Zhang, 1997 placement nov. (previously unplaced in Deltocephalinae)

Fitchana Oman, 1949

Floridonus Oman, 1949

Fridonus Oman, 1949

Garapita Oman, 1938

Gunawardenea Fletcher \& Moir, 2008

Hamulotettix Dai \& Zhang, 2011

Hardya Edwards, 1922 placement nov. (previously placed in Platymetopiina)

Hegira Oman, 1938

Houtbayana Linnavuori, 1961

Huancabamba Linnavuori, 1959

Idioceromimus Dietrich \& Rakitov, 2002 placement nov. (previously unplaced in Deltocephalinae)

Idiodonus Ball, 1936 placement nov. (previously placed in Platymetopiina)

Inghamia Evans, 1966 placement nov. (previously placed in Platymetopiina)

Jaacunga Nielson, 1988

Jakarellus Webb, 1980 placement nov. (previously placed in Platymetopiina)

Krameraxus Maldonado-Capriles, 1968 placement nov. (previously unplaced in Deltocephalinae)

Lajolla Linnavuori, 1959

Laminacutus Abdul-Nour, 2002

Lamprotettix Ribaut, 1952 placement nov. (previously placed in Platymetopiina)

Lascumbresa Linnavuori \& DeLong, 1979 
Latinocesa Kocak \& Kemal, 2010 placement nov. (previously unplaced in Deltocephalinae)

Laylatina Abdul-Nour, 1988

Lineana Li \& Xing, 2011

Lojanus Linnavuori, 1959 placement nov. (previously unplaced in Deltocephalinae)

Loralia Evans, 1966 placement nov. (transferred from Deltocephalini)

Malasiella Evans, 1954 placement nov. (previously placed in Platymetopiina)

Melillaia Linnavuori, 1971

Mesadorus Linnavuori, 1955

Mesamia Ball, 1907

Mexicananus DeLong, 1944

Mimodorus Linnavuori, 1959

Mocolinna McKamey, 2003

Moskgha Deeming \& Webb, 1982

Nakaharanus Ishihara, 1953 placement nov. (previously placed in Platymetopiina)

Napo Linnavuori \& DeLong, 1976

Neocrassana Linnavuori, 1959

Neodonus DeLong \& Hershberger, 1948 placement nov. (previously placed in Platymetopiina)

Neohegira Linnavuori \& DeLong, 1978

Neomacednus Xing \& Li, 2011

Neomesus Linnavuori, 1959

Neoreticulum Dai, 2009

Nesophryne Kirkaldy, 1907

Nesothamnus Linnavuori, 1959 placement nov. (transferred from Scaphytopiini)

Neurotettix Matsumura, 1914

Nigridonus Oman, 1949

Norvellina Ball, 1931 placement nov. (previously placed in Platymetopiina)

Nurenus Oman, 1949 placement nov. (previously placed in Platymetopiina)

Occiplanocephalus Evans, 1941

Okaundua Linnavuori, 1969

Ollarianus Ball, 1936

Ophionotum Emeljanov, 1964 placement nov. (previously placed in Allygidiina)

Orientus DeLong, 1938

Oxytettigella Metcalf, 1952

Pachytettix Linnavuori, 1959

Paracolladonus Nielson, 1988

Paracrassana Nielson, 1988

Paraganus Linnavuori, 1955

Paralaca Lozada, 1998 placement nov. (previously unplaced in Deltocephalinae)

Paramacrosteles Dai, Li \& Chen, 2006

Paranurenus Nielson, 1988

Paratanus Young, 1957

Perundanus Zanol, 1993 placement nov. (previously unplaced in Deltocephalinae)

Phlepsanus Oman, 1949

Phlepsobahita Linnavuori, 1959

Phycotettix Haupt, 1929 placement nov. (transferred from Fieberiellini)

Pingellus Evans, 1966

Platymetopius Burmeister, 1838 placement nov. (previously placed in Platymetopiina)

Poliona Emeljanov, 1972 placement nov. (previously placed in Platymetopiina)

Protensus Zhang \& Dai, 2001

Pseudalaca Linnavuori, 1959 placement nov. (previously unplaced in Deltocephalinae) 
Pseudaligia Kramer \& DeLong, 1968

Pseudaraldus Bonfils, 1981

Pseudocephalelus Linnavuori, 1969

Pseudosubhimalus Ghauri, 1974

Pseutettix DeLong, 1967

Pygotettix Matsumura, 1940

Renonus DeLong, 1959

Retusanus DeLong, 1945

Rinconada Linnavuori \& DeLong, 1977

Sanuca DeLong, 1980

Sardius Ribaut, 1946

Saudallygus Dlabola, 1979 placement nov. (previously unplaced in Deltocephalinae)

Scaphetus Evans, 1966

Scaphomonoides Li, 2011

Scaphytoceps Dlabola, 1957 placement nov. (transferred from Scaphytopiini)

Scenergates Emeljanov, 1972 placement nov. (previously placed in Platymetopiina)

Selachina Emeljanov, 1962

Serratus Linnavuori, 1959

Sincholata DeLong, 1982 placement nov. (previously unplaced in Deltocephalinae)

Sobara Oman, 1949 placement nov. (previously unplaced in Deltocephalinae)

Spinulana DeLong, 1967

Stenometohardya Dlabola, 1981

Stenomisella Evans, 1954

Stoneana DeLong, 1943

Streptopyx Linnavuori, 1958

Stymphalella Evans, 1954 placement nov. (previously placed in Platymetopiina)

Tambocerus Zhang \& Webb, 1996

Tapetia Emeljanov, 1964 placement nov. (previously placed in Platymetopiina)

Tenuisanus DeLong, 1944

Thanomahia Dlabola, 1987 placement nov. (previously unplaced in Deltocephalinae)

Tingolix Linnavuori \& DeLong, 1978

Twiningia Ball, 1931 placement nov. (previously placed in Platymetopiina)

Usanus DeLong, 1947

Watanabella Vilbaste, 1969

Yungasia Linnavuori, 1959

Zabrosa Oman, 1949

Zercanus Dlabola, 1965

Zilkaria de Menezes, 1974

Athysanus-group (informal genus group)

Fig. 11

\section{Diagnosis}

The Athysanus-group can be identified by their relatively large and broad shape, frontoclypeus often broad (never long and narrow), clypellus usually parallel-margined or sometimes slightly expanded apically, first valvula often with imbricate sculpturing pattern, and second valvulae often with distinct median dorsal tooth on shaft. 


\section{Description}

HEAD. Head subequal to or wider than pronotum. Discal portion of crown glabrous with radial or longitudinal striae, or shagreen. Anterior margin of head shagreen; crown rounded to face. Frontoclypeus not tumid to somewhat tumid; texture shagreen or glabrous. Clypellus parallel-sided, tapering apically, or widening apically; apex following or slightly surpassing normal curve of gena. Lorum subequal to or wider than clypellus near base or distinctly narrower than clypellus near base. Antennal bases near middle or posteroventral (lower) corners of eyes. Antennae short, less than $1.5 \mathrm{x}$ width of head. Gena obtusely incised laterally; with fine erect seta beside laterofrontal suture. Antennal ledges absent or weakly developed (carinate or weakly carinate). Ocelli present; close to eyes; on anterior margin of head.

THORAX. Pronotum lateral margin carinate; lateral margin shorter than half basal width of eye.

WiNGS. Forewings brachypterous to macropterous; appendix absent, reduced; or restricted to anal margin; with 3 anteapical cells; veins not raised; without reflexed costal veins; A1-A2 crossvein absent or present; apical venation not highly reticulate.

Legs. Profemur with AM1 seta only; intercalary row with one row of five or more fine setae; row AV with short, stout setae or with relatively long macrosetae. Protibia dorsal surface rounded, convex. Metafemur apex macrosetae $2+2+1$. Metatarsomere I not expanded apically or expanded apically; plantar setae simple, tapered.

MaLe Genitalia. Valve articulated with pygofer or rarely fused to subgenital plates; with short point of articulation with pygofer. Pygofer dorsoapical margin incised to near mid-length; basolateral membranous cleft present; macrosetae well differentiated into several rows. Subgenital plates free from each other; usually articulated with valve; without macrosetae or with macrosetae scattered, irregularly arranged, uniseriate laterally, with two lateral rows of macrosetae, with some irregularly arranged macrosetae near lateral margin, or macrosetae uniseriate, distant from lateral margin. Style broadly bilobed basally; median anterior lobe pronounced. Basal processes of the aedeagus/connective absent or reduced. Aedeagus without basal hinge; with a single shaft and gonopore. Connective anterior arms somewhat divergent, $\mathbf{Y}$ - or $\mathbf{U}$-shaped; articulated with aedeagus.

Female Genitalia. Pygofer with numerous macrosetae. Ovipositor not protruding far beyond pygofer apex. First valvula convex or not strongly convex; dorsal sculpturing pattern imbricate (with overlapping scales); sculpturing reaching dorsal margin; without distinctly delimited ventroapical sculpturing. Second valvula broad, gradually tapered; often with dorsal median tooth; teeth on apical 1/3 or more; teeth small, regularly or irregularly shaped.

\section{Geography and ecology}

Distribution: Palearctic, Nearctic and Oriental regions. The Athysanus-group genera feed on a variety of woody or herbaceous dicots, grasses or sedges.

\section{Remarks}

This informal genus group contains 34 genera, including genera previously included in various subtribes established for the Palearctic fauna by Emeljanov (1999) and Dmitriev (2006b). Our phylogenetic analyses suggest that some members of Allygidiina (e.g., Allygidius) and Platymetopiina (e.g., Thamnotettix) are closely related to Athysanus and/or other genera previously placed in Athysanina (e.g., Euscelis). Additionally, the circumscription of Platymetopiina by Dmitriev (2006b) contains genera that are not closely related to each other based on those included in our analyses. Thus, the previous subtribal classification is abandoned here. Also, because our analyses placed Anoterostemma in this group, Anoterostemmini, which has previously been placed in Cicadellinae (Oman et al. 1990) or as 
a subtribe of Limotettigini (Dmitriev 2002), is here synonymized with Athysanini. The representatives of our proposed Athysanus group were recovered as a monophyletic group in some, but not all, of our phylogenetic analyses and this group is diagnosable based on the morphological features listed above. More taxon sampling and more data are needed to further test the monophyly of this group. Non-Palearctic genera conforming to the above diagnosis but not considered by Emeljanov (1999) and Dmitriev (2006b) are also included in the genus group here.

\section{Selected references}

Emeljanov (1999), Dmitriev (2006b).

\section{Included genera}

Albicostella Ishihara, 1953

Allygidius Ribaut, 1948 placement nov. (previously placed in Allygidiina)

Allygus Fieber, 1872 placement nov. (previously placed in Allygidiina)

Anoterostemma Löw, 1885 placement nov. (previously placed in Anoterostemmina)

Artianus Ribaut, 1942

Athysanus Burmeister, 1838

Bilusius Ribaut, 1942

Bobacella Kusnezov, 1929

Condylotes Emeljanov, 1959 placement nov. (transferred from Paralimnini)

Conosanus Osborn \& Ball, 1902

Coulinus Beirne, 1954

Doliotettix Ribaut, 1942

Ederranus Ribaut, 1942

Euscelidius Ribaut, 1942

Euscelis Brulle, 1832

Extrusanus Oman, 1949

Graphocraerus Thomson, 1869 placement nov. (previously placed in Allygidiina)

Handianus Ribaut, 1942

Hesium Ribaut, 1942

Laburrus Ribaut, 1942

Macustus Ribaut, 1942

Matsumurella Ishihara, 1953

Mimallygus Ribaut, 1948

Mimohardya Zachvatkin, 1946

Phaeida Emeljanov, 1962

Pithyotettix Ribaut, 1942

Rhytistylus Fieber, 1875

Sotanus Ribaut, 1942

Speudotettix Ribaut, 1942

Stictocoris Thomson, 1869 placement nov. (previously placed in Allygidiina)

Streptanus Ribaut, 1942

Syringius Emeljanov, 1966 placement nov. (previously placed in Allygidiina)

Thamnotettix Zetterstedt, 1838 placement nov. (previously placed in Platymetopiina)

Transcaucasica Kocak, 1981 


\section{Bahitini tribe nov. \\ urn:Isid:zoobank.org:act:92CF5376-39E5-4B5B-B478-B8BB9AA95917}

Fig. 12

Type genus: Bahita Oman, 1938.

\section{Diagnosis}

Bahitini are medium to large sized, robust leafhoppers, often brown in color. They can be identified by the head as wide as or wider than the pronotum, crown usually short and broad and often with the anterior margin distinctly upturned, raised, or with several transverse carinae, the clypellus widening apically, parallel-margined, or distinctly tumid and tapering apically, the forewing often with strongly pigmented reflexed costal veins, the pygofer often with processes, and the aedeagus often with apical processes.

\section{Description}

HEAD. Head subequal to or wider than pronotum; head usually short and broad. Discal portion of crown glabrous with radial or longitudinal striae. Anterior margin of head with one to several transverse carinae or striae, or shagreen; usually upturned or distinctly elevated above discal portion of crown. Frontoclypeus not tumid or somewhat tumid; texture shagreen. Clypellus tapering apically, parallel-sided, or widening apically; sometimes bulbous and tumid; apex following or slightly surpassing normal curve of gena. Lorum subequal to or wider than clypellus near base. Antennal bases near middle or posteroventral (lower) corners of eyes. Antennae short, less than $1.5 \mathrm{x}$ width of head. Gena obtusely incised laterally; with fine erect seta beside laterofrontal suture. Antennal ledges absent or weakly developed (carinate or weakly carinate). Ocelli present; close to eyes; on anterior margin of head.

Thorax. Pronotum lateral margin carinate; lateral margin shorter than basal width of eye.

Wings. Forewing macropterous; appendix restricted to anal margin; with 3 anteapical cells; veins not raised; veins often heavily marked with brown coloration; often with or sometimes without pigmented reflexed costal veins; sometimes with extra reflexed costal veins; A1-A2 crossvein absent or present; apical venation not highly reticulate.

LEgs. Profemur with AM1 seta only or rarely with an extra macroseta basad of AM1; intercalary row with one row of five or more fine setae; row AV with short, stout setae. Protibia dorsal surface rounded, convex. Metafemur apex macrosetae $2+2+1$. Metatarsomere I not expanded apically; plantar setae simple, tapered.

MALE GENITALIA. Valve articulated with pygofer; lateral margin short, articulating with pygofer at a point. Pygofer basolateral membranous cleft present; macrosetae well differentiated into several rows; often with one or more spines or processes arising ventrally. Subgenital plates free from each other; articulated with valve; macrosetae uniseriate laterally, sometimes with few other scattered macrosetae. Style broadly bilobed basally; median anterior lobe pronounced. Aedeagus with single shaft and gonopore; often with pair of apical processes. Connective anterior arms somewhat divergent, Y-shaped, or rarely with anterior arms closely appressed (e.g., Menosoma); articulated with aedeagus.

Female Genitalia. Pygofer with numerous macrosetae. Ovipositor not protruding far beyond pygofer apex. First valvula convex; dorsal sculpturing pattern strigate; sculpturing reaching dorsal margin; without distinctly delimited ventroapical sculpturing. Second valvula broad, gradually tapered; with or without dorsal median tooth; teeth on apical $1 / 3$ or more; teeth small, regularly or irregularly shaped. 


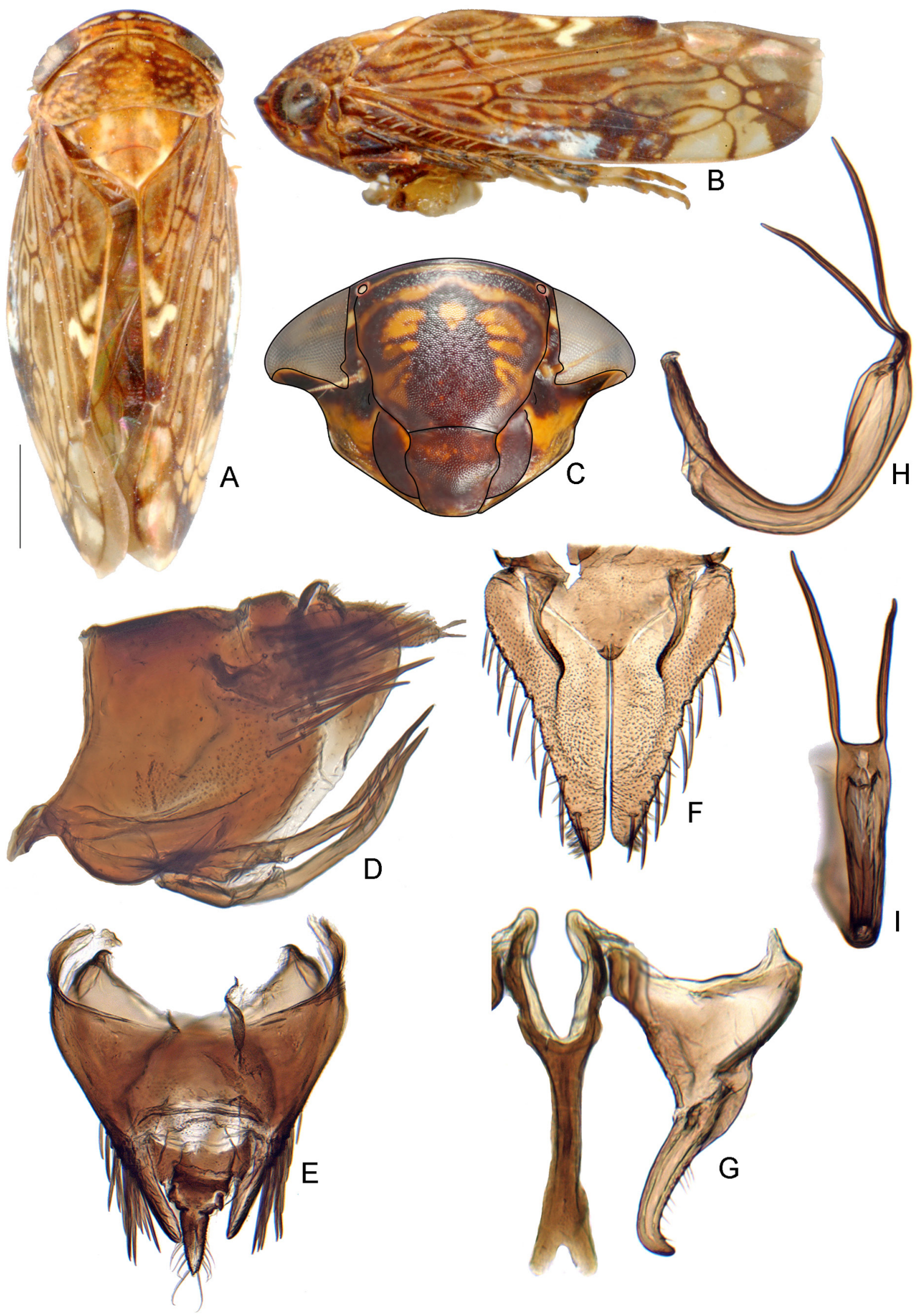

Fig. 12. Bahitini. A-B, D-I. Bahita infuscata (Osborn, 1923). C. Bahita mimica Linnavuori \& DeLong, 1978. A-I. Standard views (see Material \& Methods). 


\section{Geography and ecology}

Distribution: Neotropical and Nearctic regions. This group is particularly diverse in Neotropical forested habitats.

\section{Remarks}

Bahitini contains 25 genera and 166 species. This is an exclusively New World group as currently understood. Although the structure of the head, particularly the presence of more than one transverse carina or striation at the anterior margin of the crown, gives some members of this group a superficial resemblance to the Old World tribe Selenocephalini, our phylogenetic analyses did not indicate a close relationship between these groups. Some other New World athysanine genera are similar in having the anterior margin of the head upturned and with reflexed costal veins (e.g., Lajolla, Bardana, Acunasus) but these are generally more elongate, more slender and/or have a more produced head which might suggest that they are more related to the Platymetopius/Twiningia group. More detailed analyses of the relationships of the genera of Athysanini may indicate whether some of these should be included into this tribe. Benibahita was included in the Bahita-group by Linnavuori \& DeLong (1978b) but is excluded here, as it appears to be more closely related to Yungasia (Athysanini; similar color pattern, with head narrower than pronotum, more slender in shape smaller in size than Bahitini). Angubahita DeLong is excluded based on its small size and absence of other characteristics of the group. The phylogenetic analyses presented here resolved a monophyletic group including representatives of Bahita, Caruya, Kinrentius, Menosoma, Taperinha, and Oxycephalotettix. Further taxonomic and phylogenetic study of the Neotropical deltocephaline fauna may refine the circumscription of this group.

\section{Selected references}

Linnavuori \& DeLong (1978b), Zanol (1999).

\section{Included genera}

Bahita Oman, 1938 placement nov. (transferred from Athysanini)

Brincadorus Oman, 1938 placement nov. (transferred from Athysanini)

Caruya Linnavuori \& DeLong, 1978 placement nov. (transferred from Athysanini)

Chonosina Linnavuori \& DeLong, 1978 placement nov. (transferred from Athysanini)

Concepciona Linnavuori \& DeLong, 1977 placement nov. (transferred from Athysanini)

Frequenamia DeLong, 1947 placement nov. (transferred from Athysanini)

Hecaloidia Osborn, 1923 placement nov. (transferred from Athysanini)

Huachia Linnavuori, 1959 placement nov. (transferred from Athysanini)

Ilagia Kramer \& DeLong, 1968 placement nov. (transferred from Athysanini)

Kinrentius Wei, Dietrich \& Webb, 2010 placement nov. (previously unplaced in Deltocephalinae)

Kyra Zanol, 2011 placement nov. (transferred from Athysanini)

Megabahita Linnavuori \& DeLong, 1978 placement nov. (transferred from Athysanini)

Menosoma Ball, 1931 placement nov. (transferred from Athysanini)

Oxycephalotettix Zahniser, 2005 placement nov. (transferred from Athysanini)

Parabahita Linnavuori, 1959 placement nov. (transferred from Athysanini)

Perubahita Linnavuori \& DeLong, 1978 placement nov. (transferred from Athysanini)

Rineda Linnavuori \& DeLong, 1978 placement nov. (transferred from Athysanini)

Saadevra McKamey, 2003 placement nov. (transferred from Athysanini)

Sanestebania Linnavuori \& DeLong, 1978 placement nov. (transferred from Athysanini)

Serridonus Linnavuori, 1959 placement nov. (transferred from Athysanini)

Sinchonoa Linnavuori \& DeLong, 1978 placement nov. (transferred from Athysanini)

Suarezilinna McKamey, 2003 placement nov. (transferred from Athysanini)

Taperinha Linnavuori, 1959 placement nov. (transferred from Athysanini) 
Tingopyx Linnavuori \& DeLong, 1978 placement nov. (transferred from Athysanini)

Yuraca Linnavuori \& DeLong, 1978 placement nov. (transferred from Athysanini)

Bonaspeiini tribe nov.

urn:1sid:zoobank.org:act:50F524A4-754D-4EA4-8466-98A20D4DFA84

Fig. 13

Type genus: Bonaspeia Linnavuori, 1961.

\section{Diagnosis}

Bonaspeiini are medium sized, broad, robust, brachypterous to submacropterous, ivory, greenish, or brownish leafhoppers. They can be identified by the anterior margin of the head without sharp carinae (with sharp carinae in Selenocephalini), brachypterous to submacropterous forewings (exception: Renosteria), hind wings when present narrowed anteriorly-posteriorly when outstretched or otherwise reduced in size (exception: Renosteria), connective U- or sometimes Y- shaped with stem often very short or absent, male segment $\mathrm{X}$ broad and sclerotized, and ovipositor usually distinctly protruding beyond the pygofer apex.

\section{Description}

HeAd. Head subequal to or wider than pronotum. Discal portion of crown glabrous with radial or longitudinal striae. Anterior margin of head shagreen, irregularly textured, or with several to numerous transverse striae. Frontoclypeus not tumid; texture shagreen, glabrous, or rugose. Clypellus parallel sided, tapering apically, or widening apically; apex following or slightly surpassing normal curve of gena. Lorum subequal to or wider than clypellus near base. Antennal bases near middle or posteroventral (lower) corners of eyes or near upper or anterodorsal corners of eyes. Antennae short, less than $1.5 \mathrm{x}$ width of head. Gena incised laterally; with fine erect seta beside laterofrontal suture. Antennal ledges weakly developed (carinate or weakly carinate). Ocelli often reduced in size; close to or distant from eyes; on anterior margin of head or shifted slightly dorsad on crown.

THORAX. Pronotum lateral margin not carinate or carinate; lateral margin shorter than basal width of eye.

Wings. Forewing brachypterous to submacropterous, or rarely macropterous (Renosteria); with 3 anteapical cells; veins not raised; with or without reflexed costal veins; A1-A2 crossvein absent or present; apical venation not highly reticulate. Hind wing, when present, narrowed anteriorly-posteriorly when outstretched or otherwise reduced in size; with full complement of wing veins or with some veins (e.g., $\mathrm{M} 3+4$ and $\mathrm{CuA}$ ) fused.

Legs. Profemur with AM1 seta only; intercalary row with one row of 5 or more fine setae; row AV with short, stout macrosetae or with relatively long macrosetae. Protibia dorsal surface rounded, convex. Metafemur apex macrosetae $2+2+1$. Metatarsomere I not expanded or expanded apically; plantar setae all simple or rarely (e.g., some Gcaleka) one or more platellae.

Male Genitalia. Valve articulated with pygofer; with short point of articulation with pygofer. Pygofer dorsoapical margin incised to near mid-length or nearly to base; basolateral membranous cleft present, sometimes sharply angulate; macrosetae absent or reduced ( $\leq$ two rows) or rarely well differentiated into several rows; often with spine-like process. Subgenital plates free from each other; articulated with valve; macrosetae scattered, irregularly arranged, uniseriate laterally, with two or more lateral rows of macrosetae, or with some irregularly arranged macrosetae near lateral margin. Style linear, median anterior lobe not pronounced or broadly bilobed basally, median anterior lobe pronounced. Basal processes of the aedeagus/connective absent or reduced or present, connected or articulated to 


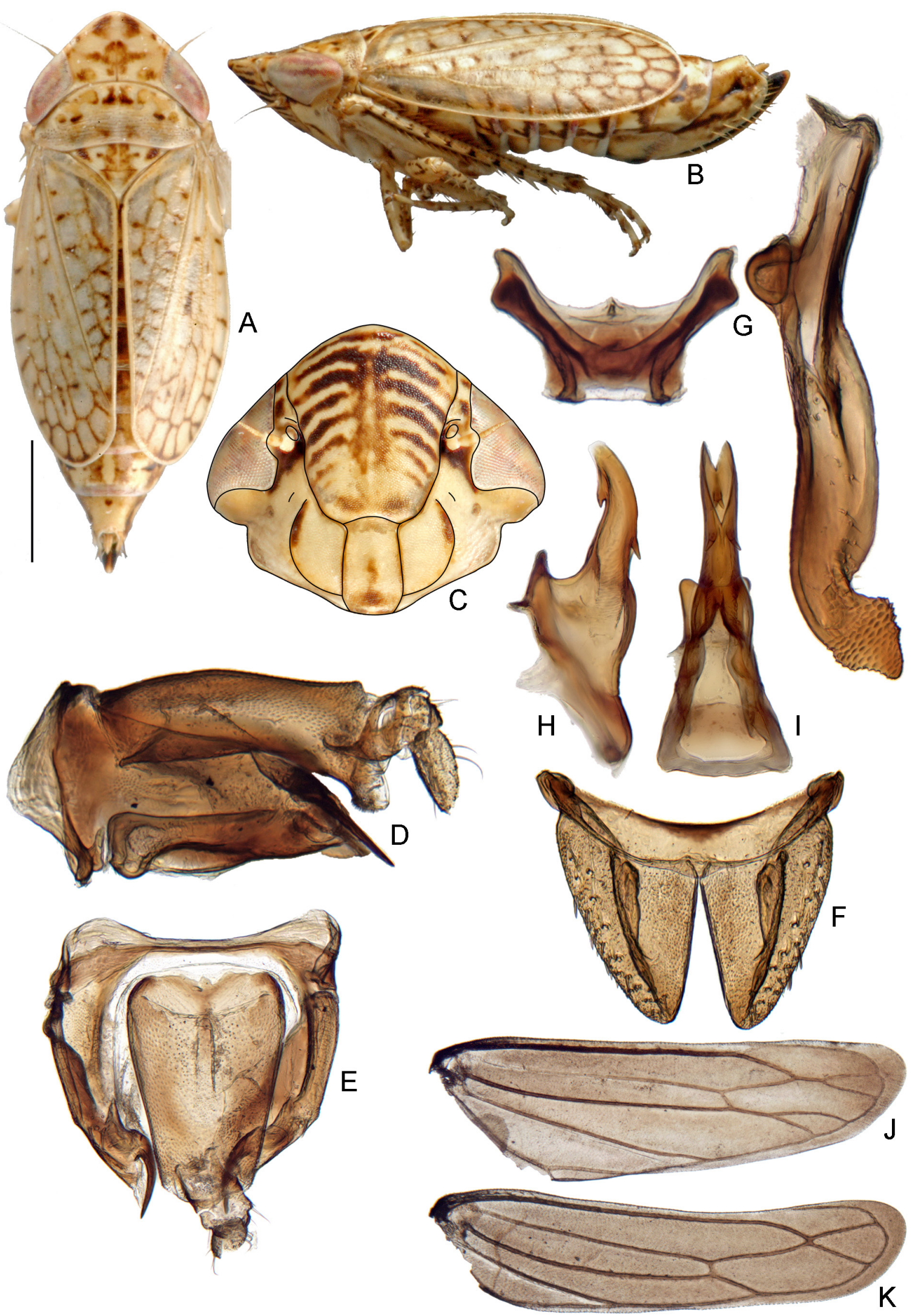

Fig. 13. Bonaspeiini. A-I. Standard views (see Material \& Methods), Bonaspeia attia Davies, 1987. J-K. Hind wing. J. Cerus goudanus Theron, 1984. K. Curvostylus chloridulus (Naudé, 1926). 
connective or near base of aedeagus. Aedeagus without basal hinge; with a single shaft and gonopore; often with short hooks or spines. Connective anterior arms somewhat divergent, U- or sometimes Yshaped; stem often very short or absent; articulated with aedeagus. Segment X broad, sometimes very large and occupying much of dorsal part of pygofer; sclerotized dorsally and laterally.

Female genitalia. Pygofer with macrosetae reduced or absent or with numerous macrosetae. Ovipositor not protruding or protruding far beyond pygofer apex. First valvula convex, dorsal sculpturing pattern strigate, concatenate, or reticulate; sculpturing reaching dorsal margin; without distinctly delimited ventroapical sculpturing. Second valvula broad, gradually tapered; with or without dorsal median tooth; teeth on apical $1 / 3$ or more; teeth small, regularly or irregularly shaped.

\section{Geography and ecology}

Distribution: Afrotropical (South Africa). Bonaspeiini are common in the fynbos biome and collected on woody and herbaceous dicots. Gcaleka species are dorsoventrally flattened, apparently an adaptation to feeding on their reed-like hosts in the family Restionaceae. Salsolibia and Salsocolila feed on the ganna bush, Salsola esterhuyseniae Botsch. (Amaranthaceae), a woody-stemmed bush with succulent leaves living in silt deposits in valleys and riverbeds. The squat, compact leafhoppers apparently mimic the succulent leaves of their host (Theron 1979). Renosteria, Cerus and Refrolix feed on renosterbos, Elytropappus rhinocerotis Less. (Asteraceae), a common plant in the southern part of the Western Cape Province. Davies (1988a) reports Kimbella acusiculcita Davies, 1988 from Erica peltata Andrews (Ericaceae) and Colistra parvulus (Linnavuori, 1961) from Erica hispudula L. and Erica muscosa (Aiton) E.G.H.Oliv.

\section{Remarks}

Bonaspeiini contains 21 genera and 108 species. The tribe is closely related to Selenocephalini but is considered distinct here due to the lack of distinct carinae on the anterior margin of the head which are typical of most Selenocephalini and the presence of a number of unique characters (see Diagnosis). Three exemplars (from Bonaspeia, Cerus, and Renosteria) were included in the phylogenetic analyses here, and the group was resolved as paraphyletic with respect to the clade Selenocephalini + Dagama (Athysanini). However, branch support was not high, and more detailed phylogenetic analyses are needed to further resolve these relationships. A representative of Gcaleka was included in previous analyses (Zahniser \& Dietrich 2010) and was resolved as sister to Bonaspeia, but was excluded from analyses here due to missing data in the $28 \mathrm{~S}$ sequence.

\section{Selected references}

Linnavuori (1961), Theron (1974, 1984a, b), Stiller (1986), Davies (1987, 1988a, b).

\section{Included genera}

Basutoia Linnavuori, 1961 placement nov. (transferred from Athysanini)

Bloemia Theron, 1974 placement nov. (transferred from Athysanini)

Bonaspeia Linnavuori, 1961 placement nov. (previously unplaced in Deltocephalinae)

Caffrolix Linnavuori, 1961 placement nov. (transferred from Athysanini)

Capeolix Linnavuori, 1961 placement nov. (transferred from Athysanini)

Cerus Theron, 1975 placement nov. (previously unplaced in Deltocephalinae)

Colistra Davies, 1988 placement nov. (transferred from Athysanini)

Curvostylus Davies, 1987 placement nov. (transferred from Athysanini)

Gcaleka Naudé, 1926 placement nov. (previously unplaced in Deltocephalinae)

Hadroca Theron, 1974 placement nov. (previously unplaced in Deltocephalinae)

Johanus Theron, 1974 placement nov. (transferred from Athysanini) 
Kaapia Theron, 1983 placement nov. (transferred from Athysanini)

Kimbella Davies, 1988 placement nov. (transferred from Athysanini)

Megaulon Theron, 1975 placement nov. (transferred from Paralimnini)

Proekes Theron, 1975 placement nov. (transferred from Stenometopiini)

Proekoides Stiller, 1986 placement nov. (transferred from Stenometopiini)

Refrolix Theron, 1984 placement nov. (transferred from Athysanini)

Renosteria Theron, 1974 placement nov. (transferred from Athysanini)

Salsocolila McKamey, 2003 placement nov. (transferred from Athysanini)

Salsolibia Theron, 1979 placement nov. (transferred from Athysanini)

Tzitzikamaia Linnavuori, 1961 placement nov. (transferred from Athysanini)

\section{Chiasmini Distant, 1908}

Fig. 14

Type genus: Chiasmus Mulsant \& Rey, 1855.

$=$ Doraturini Emeljanov, 1962.

$=$ Listrophorini Boulard, 1971 syn. nov.

$=$ Paraphrodini Linnavuori, 1979.

\section{Diagnosis}

Chiasmini are small to medium sized leafhoppers, usually white, stramineous, green, brown, grey, or black in coloration, and sometimes iridescent. They can be identified by the tapering or parallelsided clypellus, aedeagus hinged at the base (hinge usually but not always present), ovipositor usually extending far beyond the pygofer, first valvula dorsal sculpturing pattern maculose to granulose and usually submarginal, first valvula without distinctly delimited ventroapical sculpturing, and second valvula teeth obliquely triangular and serrated.

\section{Description}

HeAD. Head subequal to or wider than pronotum. Discal portion of crown glabrous with radial or longitudinal striae or shagreen. Anterior margin of head shagreen, glabrous, irregularly textured, or foliaceous. Frontoclypeus not tumid; texture shagreen or glabrous. Clypellus parallel-sided or tapering apically; apex following or slightly surpassing normal curve of gena. Lorum wider than, subequal to, or distinctly narrower than clypellus near base. Antennal bases near middle or posteroventral (lower) corners of eyes. Antennae short, less than $1.5 \mathrm{x}$ width of head. Gena obtusely incised or strongly incised (nearly forming right angle) laterally; with or without (Baileyus, Gurawa, Listrophora, some Chiasmus) fine erect seta beside laterofrontal suture. Antennal ledge absent or weakly developed (carinate or weakly carinate). Ocelli absent, reduced, or present; close to or distant from eyes; on anterior margin of head or on crown (Chiasmus).

THORAX. Pronotum lateral margin carinate or not carinate; lateral margin shorter than basal width of eye.

WINGS. Forewing brachypterous to macropterous; if macropterous then appendix large and extending around wing apex; with 3 anteapical cells or with 2 anteapical cells; veins not raised or veins distinctly raised or carinate (Baileyus, Gurawa, Paraphrodes); without reflexed costal veins; A1-A2 crossvein absent.

LeGs. Profemur with AM1 seta only or rarely with an additional proximal seta (Doraturopsis Lindberg, Protochiasmus Zahniser); intercalary row with one row of five or more fine setae or intercalary row reduced or absent; row AV with short, stout setae or with relatively long macrosetae. Protibia dorsal 


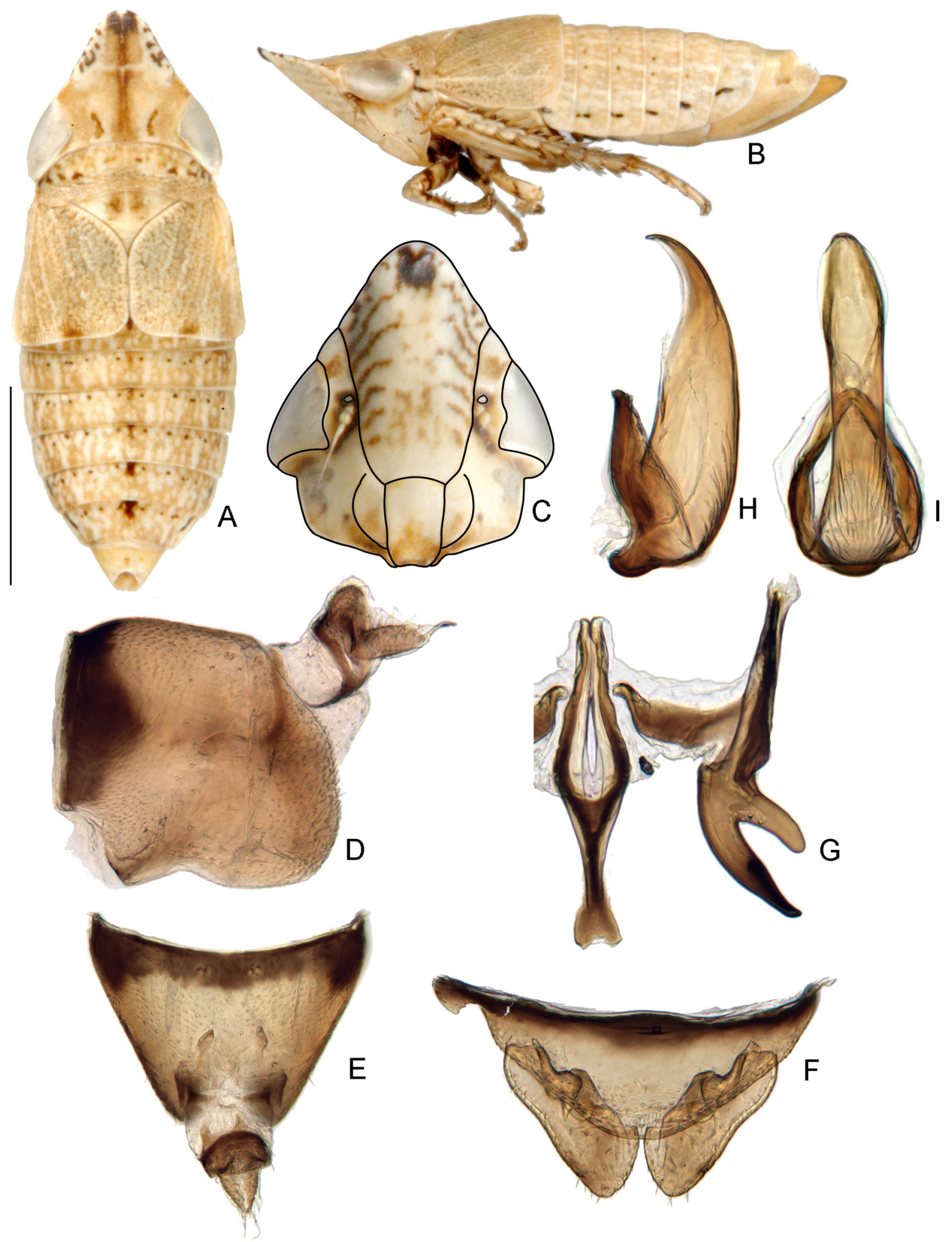

Fig. 14. Chiasmini. Chiasmus sp. (see note under Chiasmini on identification of species of Chiasmus). A-I. Standard views (see Material \& Methods). 
surface rounded, convex. Metafemur apex macrosetae $2+1,2+2,2+1+1$, or $2+2+1$. Metatarsomere I not expanded apically or expanded apically; plantar setae simple, tapered.

MALE GENITALIA. Valve articulated with pygofer; lateral margin short, articulating with pygofer at a point. Pygofer basolateral membranous cleft present or absent, not membranous; macrosetae absent, reduced ( $\leq$ two rows) or well differentiated into several rows. Subgenital plates free from each other; articulated with valve; macrosetae absent or present, scattered, irregularly arranged, or uniseriate laterally. Style broadly bilobed basally, median anterior lobe pronounced. Basal processes of the aedeagus/connective absent or reduced. Aedeagus often with or sometimes without (Baileyus, Leofa, Gurawa, Paraphrodes) basal hinge; with a single shaft and gonopore. Connective anterior arms somewhat divergent, Y- or U-shaped, or anterior arms closely appressed anteriorly; articulated with aedeagus.

Female genitalia. Pygofer with macrosetae reduced or absent or with numerous macrosetae. Ovipositor usually protruding far beyond pygofer apex (not protruding in Baileyus, Gurawa, Omaranus, Paraphrodes). First valvula not strongly convex; dorsal sculpturing pattern granulose or maculose; sculpturing submarginal; without distinctly delimited ventroapical sculpturing. Second valvula abruptly broadened medially or subapically; without dorsal median tooth; dorsal teeth present on apical 1/3 or more; teeth obtusely triangular and serrated.

\section{Geography and ecology}

Distribution: cosmopolitan. Nearly all species are grass or sedge feeders, and they are typical members of the grassland faunas of the world. Driotura spp. have been collected from Asteraceae. Nephotettix virescens (Distant, 1908) and some other Nephotettix spp. are agricultural pests and transmit tungro virus of rice in southeast Asia, and can cause severe economic loss.

\section{Remarks}

Chiasmini contains 21 genera and 317 species. The tribe appears to be most closely related to Stenometopiini (Zahniser 2008a; Zahniser \& Dietrich 2010) with which it shares some similarities of the ovipositor and male pygofer. Phylogenetic analyses sometimes resolve it as sister to Stenometopiini, and also suggest that the two tribes are related to Eupelicini, Drakensbergenini, and Evinus. In the phylogenetic analyses here, the tribe was resolved as monophyletic in ML and Bayesian analyses, but in parsimony analyses it was monophyletic with the exception of Protochiasmus, which was resolved, sister to Evinus but with little or no branch support. The placement of Gurawa in Chiasmini is supported by these analyses and the morphologically similar Baielyus and Paraphrodes (Zahniser 2008a, 2011) are also included.

For the first time, molecular data for Listrophora were included in phylogenetic analyses and the genus was found with very strong branch support to be related to Chiasmini. The morphological characters of the male and female genitalia of Listrophora (aedeagus hinged, first valvula dorsal sculpturing pattern granulose and submarginal, second valvula with distinct serrated oblong and triangular teeth) match those of other Chiasmini, despite the bizarre and unique diamond-shaped process of the head, which led Boulard (1971) to place it in a separate tribe. In our analyses it was found to be closely related to Gurawa within Chiasmini, with which it shares the raised or carinate veins of the forewing. Listrophorina was previously included as a subtribe of Eupelicini (Zahniser \& Dietrich 2010) but is synonymized here with Chiasmini.

Nephoris is transferred to the tribe based on the figures published by Jacobi (1912). N. chalybaea Jacobi, 1912 appears similar to Leofa thompsoni Zahniser, 2008 which was also described from central Africa. Attempts to locate the type were unsuccessful, but for now it is considered a valid genus pending further study. 


\section{Selected references}

Vilbaste (1965), Ross (1968), Boulard (1971),Blocker (1983), Blocker \& Johnson (1988a, b, 1990a, b, c), Emeljanov (1999), Dmitriev (2003), Zahniser \& Hicks (2007), Zahniser (2008a, b, 2011), Duan et al. (2009), Duan \& Zhang (2012a, b, c).

\section{Notes on illustration (Fig. 14)}

The specimens figured are identified as Chiasmus sp. from W. Cape Province, South Africa. The species identity of Chiasmus specimens is difficult to determine given the variability in some described species and because of the relatively uniform male genitalia among species. C. varicolor (Kirkaldy, 1906) is known from Australia and South Africa, C. undulatus Theron, 1982 is also known from South Africa, and C. katonae (Melichar, 1908) is known from Tanzania. The species figured is similar to C. katonae but differs in size, surface shape of the crown, and coloration. The genus should be revised and species concepts reevaluated to improve the taxonomy and allow for easier identification.

\section{Included genera}

Aconura Lethierry, 1876

Aconurella Ribaut, 1948

Athysanella Baker, 1898

Baileyus Singh-Pruthi, 1930

Chiasmus Mulsant \& Rey, 1855

Doratura Sahlberg, 1871

Doraturopsis Lindberg, 1935

Driotura Osborn \& Ball, 1898

Exitianus Ball, 1929

Gurawa Distant, 1908

Icaia Linnavuori, 1973

Leofa Distant, 1918

Listrophora Boulard, 1971 placement nov. (previously placed in Eupelicini: Listrophorina)

Nephoris Jacobi, 1912 placement nov. (transferred from Athysanini)

Nephotettix Matsumura, 1902

Omaranus Distant, 1918

Paraphrodes Linnavuori, 1979

Picchusteles Linnavuori \& DeLong, 1976

Protochiasmus Zahniser, 2010

Stenogiffardia Evans, 1977

Zahniserius Duan \& Zhang, 2012

Cicadulini Van Duzee, 1892

Fig. 15

Type genus: Cicadula Zetterstedt, 1840.

$=$ Procepitina Dmitriev, 2002 syn. nov.

\section{Diagnosis}

Cicadulini are small to medium sized, slender, stramineous, yellow, or greenish leafhoppers, sometimes with the anterior margin of the head marked with black spots. They can be identified by the male segment $\mathrm{X}$ often long and strongly sclerotized, and subgenital plates sometimes with a row of macrosetae near the middle and with long fine setae laterally. 


\section{Description}

HEAD. Head subequal to or wider than pronotum. Discal portion of crown shagreen. Anterior margin of head shagreen. Frontoclypeus not tumid; texture shagreen. Clypellus widening apically; apex following or slightly surpassing normal curve of gena. Lorum subequal to or wider than clypellus near base. Antennal bases near middle or posteroventral (lower) corners of eyes. Antennae short, less than $1.5 \mathrm{x}$ width of head or long, $1.5 \mathrm{x}$ width of head or longer. Gena obtusely incised laterally; with fine erect seta beside laterofrontal suture. Antennal ledge absent. Ocelli present; close to or distant from eyes; on anterior margin of head.

Thorax. Pronotum lateral margin carinate; lateral margin shorter than basal width of eye.

WiNGS. Forewing macropterous; appendix restricted to anal margin; with 3 anteapical cells; veins not raised; without reflexed costal veins; A1-A2 crossvein absent.

LeGs. Profemur with AM1 seta only; intercalary row with one row of five or more fine setae; row AV with short, stout setae. Protibia dorsal surface rounded, convex. Metafemur apex macrosetae $2+2+1$. Metatarsomere I not expanded apically; plantar setae simple, tapered.

Male genitalia. Sternal apodemes of abdominal segments I and II usually well-developed. Valve articulated with pygofer; lateral margin short, articulating with pygofer at a point. Pygofer basolateral membranous cleft present or absent, not membranous; macrosetae well differentiated into several rows. Subgenital plates free from each other; articulated with valve; macrosetae uniseriate laterally or uniseriate, distant from lateral margin. Style broadly bilobed basally, median anterior lobe pronounced. Basal processes of the aedeagus/connective absent or reduced. Aedeagus with single shaft and gonopore. Connective anterior arms somewhat divergent, Y-shaped; articulated with aedeagus. Segment X long, length usually $2 \mathrm{x}$ width or longer; strongly sclerotized dorsally.

Female genitalia. Pygofer with numerous macrosetae. Ovipositor not protruding far beyond pygofer apex. First valvula convex; dorsal sculpturing pattern imbricate (with overlapping scales); sculpturing reaching dorsal margin; without distinctly delimited ventroapical sculpturing. Second valvula broad, gradually tapered; without dorsal median tooth; dorsal teeth on apical 1/3 or more; teeth small, regularly or irregularly shaped.

\section{Geography and ecology}

Distribution: Nearctic, Neotropical and Palearctic regions. Cicadulini feed on sedges and grasses, and are often found in association with wetland or marsh habitats or in forest understories.

\section{Remarks}

Cicadulini contains 14 genera and 114 valid species. This group was considered a subtribe of Athysanini by Dmitriev (2006b) and Emeljanov (1999) included cicaduline genera in his Athysanina. Two exemplars of the tribe (Cicadula and Elymana) were included in a phylogenetic analysis of Deltocephalinae (Zahniser \& Dietrich 2010) and were supported as a monophyletic group. Molecular data for Proceps and Stenometopiellus included here for the first time suggest with strong branch support that they belong in Cicadulini; they are transferred to the tribe here. Dmitriev (2002) described the monotypic Procepitina as a subtribe of Scaphytopiini, but it is considered a synonym of Cicadulini here. The characters of the ovipositor of Proceps are consistent with those of other Cicadulini; males were not available for this study but Emeljanov (1967) described male segment X as long and well sclerotized, consistent with other members of the tribe. Cicadulini is considered a tribe separate from Athysanini following Oman et al. (1990). It appears to be related to other grass-feeding tribes of Deltocephalinae and perhaps to Limotettigini, but more data are needed to more firmly resolve its relationship to other tribes. 

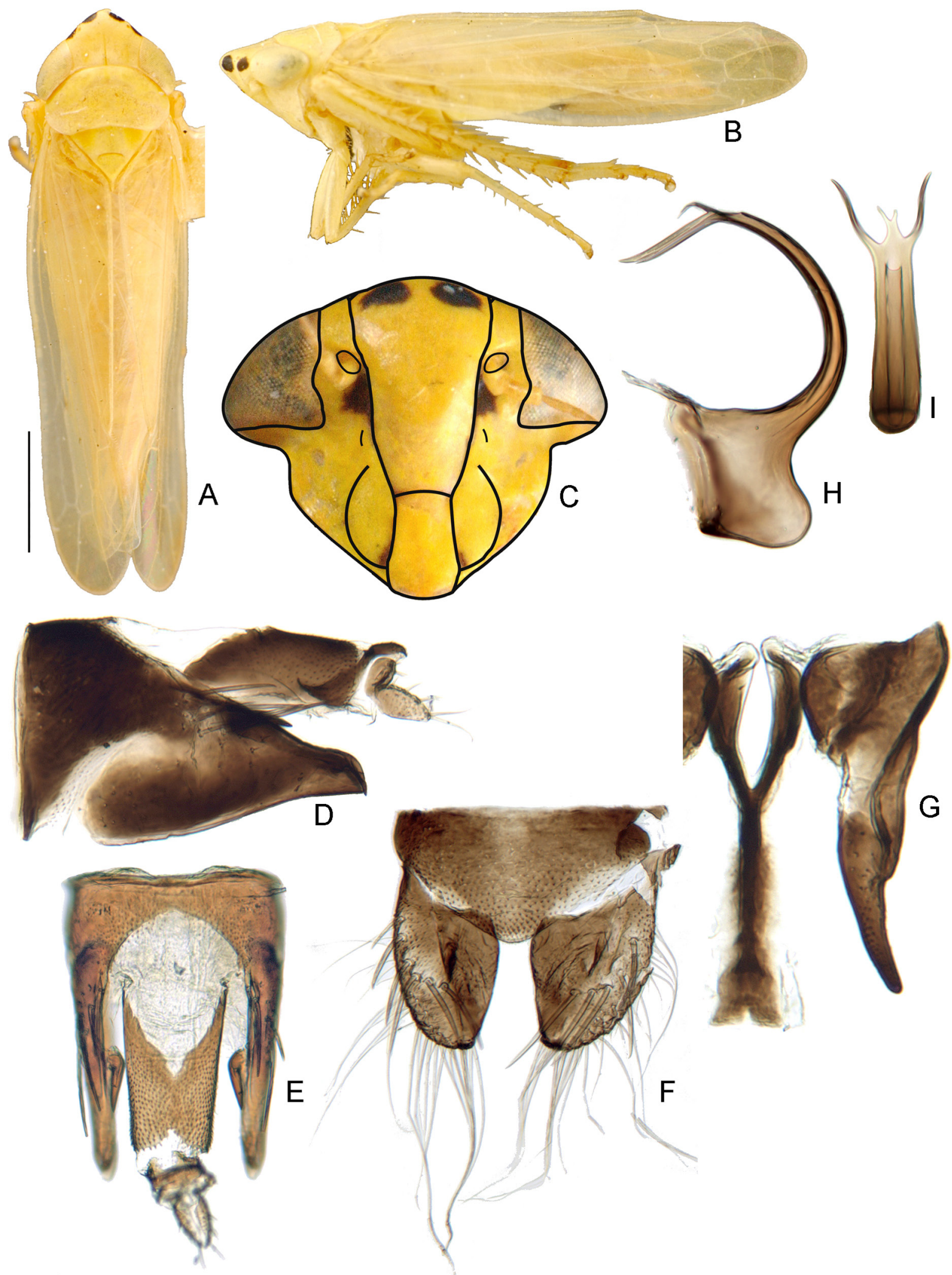

Fig. 15. Cicadulini. A-C. Cicadula melanogaster (Provancher, 1872). D-I. Cicadula quadrinotata (Fabricius, 1794). A-I. Standard views (see Material \& Methods). 
Calanana was listed in Athysanini by Oman et al. (1990). However, it was clearly suggested to be related to Cicadula, Elymana, and Paluda by Oman (1949) with which it shares the long sclerotized segment $\mathrm{X}$ and the subgenital plate with a row of macrosetae running medially along the plate. It is therefore placed in Cicadulini here.

\section{Selected references}

Hamilton (1975b), Dmitriev (2002).

\section{Included genera}

Calanana DeLong, 1945 placement nov. (transferred from Athysanini)

Cicadula Zetterstedt, 1840

Dudanus Dlabola, 1956

Elymana DeLong, 1936

Hecadula Dietrich \& Rakitov, 2002

Knullana DeLong, 1941

Mocydia Edwards, 1922

Mocydiopsis Ribaut, 1939

Morinda Emeljanov, 1972

Paluda DeLong, 1937

Proceps Mulsant \& Rey, 1855 placement nov. (previously in Scaphytopiini: Procepitina)

Rhopalopyx Ribaut, 1939

Stenometopiellus Haupt, 1917 placement nov. (transferred from Athysanini)

Taurotettix Haupt, 1929

\section{Cochlorhinini Oman, 1943}

Fig. 16

Type genus: Cochlorhinus Uhler, 1876.

\section{Diagnosis}

Cochlorhinini are medium sized leafhoppers, with ground color greenish, yellow, brown, black, or ivory. They can be identified by the Y-shaped connective fused to aedeagus, male pygofer with one or more pairs of teeth, spines, or processes, male pygofer side excavated medially and scooplike, male segment $\mathrm{X}$ long and sclerotized laterally and sometimes ventrally and/or dorsally, and second valvulae with a median dorsal tooth.

\section{Description}

Head. Head subequal to or wider than pronotum. Discal portion of crown glabrous with radial or longitudinal striae. Anterior margin of head shagreen, glabrous, irregularly textured, or foliaceous. Frontoclypeus not tumid; texture shagreen. Clypellus widening apically; apex following or slightly surpassing normal curve of gena. Lorum subequal to or wider than clypellus near base. Antennal bases near middle or posteroventral (lower) corners of eyes. Antennae short, less than $1.5 \mathrm{x}$ width of head. Gena obtusely incised laterally; with fine erect seta beside laterofrontal suture. Antennal ledge absent. Ocelli present; close to or distant from eyes; on anterior margin of head.

THORAX. Pronotum lateral margin carinate; lateral margin shorter than basal width of eye.

WINGS. Forewing macropterous or submacropterous; appendix restricted to anal margin; with 3 anteapical cells; veins not raised; without reflexed costal veins; A1-A2 crossvein absent. 


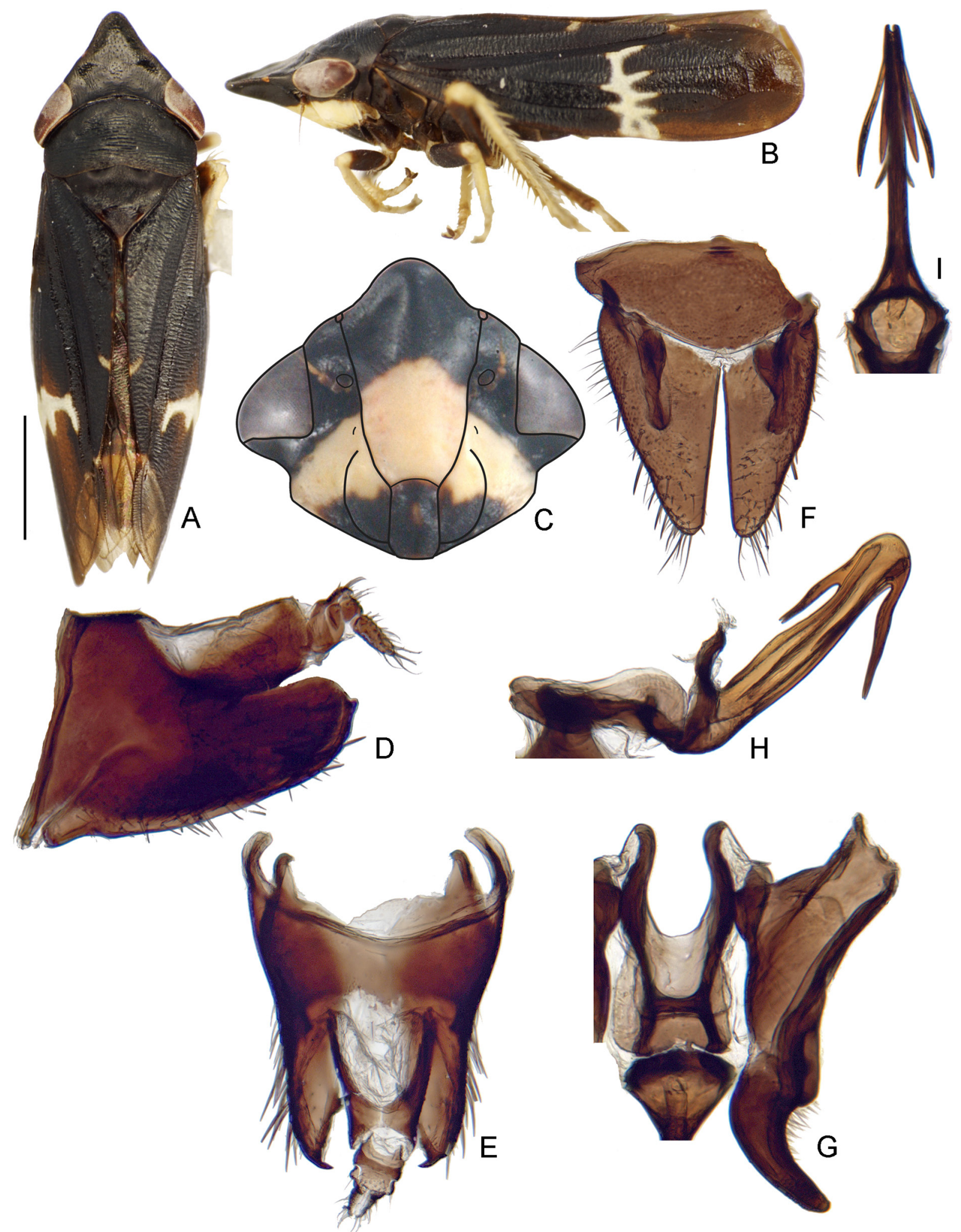

Fig. 16. Cochlorhinini. Cochlorhinus pluto Uhler, 1876. A-I. Standard views (see Material \& Methods). H-I. Aedeagus shown with connective fused at base. 
LEgs. Profemur with AM1 seta only; intercalary row with one row of five or more fine setae; row AV with short, stout setae. Protibia dorsal surface rounded, convex. Metafemur apex macrosetae $2+2+1$. Metatibia row PD macrosetae often long, nearly equal to or longer than half length of protibia. Metatarsomere I not expanded apically; plantar setae with one or more platellae or without platellae.

MaLe Genitalia. Valve articulated with pygofer; lateral margin short, articulating with pygofer at a point. Pygofer basolateral membranous cleft present; macrosetae well differentiated into several rows; dorsally excavated to mid-length or nearly to base; side excavated medially, scooplike; usually with apical or subapical teeth, spines, or processes. Subgenital plates free from each other; articulated with valve; macrosetae uniseriate laterally or irregularly arranged near lateral margin. Style broadly bilobed basally, median anterior lobe pronounced. Basal processes of the aedeagus/connective present (paired or singular) or absent. Aedeagus with single shaft and gonopore; symmetrical or asymmetrical; sometimes dorsoventrally flattened or lamellate; sometimes with 2 or 3 pairs of apical processes. Connective anterior arms somewhat divergent, $\mathbf{Y}$ - or $\mathbf{U}$-shaped; fused to aedeagus. Segment X relatively long, sclerotized laterally, and sometimes sclerotized ventrally and/or dorsally.

Female Genitalia. Pygofer with numerous macrosetae. Ovipositor not protruding far beyond pygofer apex. First valvula convex; dorsal sculpturing pattern strigate, concatenate, or reticulate; sculpturing reaching dorsal margin; without distinctly delimited ventroapical sculpturing. Second valvula broad, gradually tapered; usually with dorsal median tooth; dorsal teeth on apical $1 / 3$ or more; teeth small, regularly or irregularly shaped.

\section{Geography and ecology}

Distribution: Nearctic (western US), Neotropical (Cochlorhinus pluto Uhler, 1876, introduced in Chile). All are endemic to the western United States and are collected in grasslands, meadows, and forest edges and understories.

\section{Remarks}

Cochlorhinini contains 12 genera and 145 species. Phylogenetic analyses recovered Ballana as sister to Cochlorhinus with moderate to strong branch support. Based on this result and on shared morphological characters (see Diagnosis above) and a western North American distribution, Allygianus, Allygiella, Ballana, Gloridonus, Limbanus, and Pasadenus are transferred to Cochlorhinini here. These genera do not possess some characters that previously helped to distinguish a more strict interpretation of the tribe (sensu Oman 1949): ocelli distant from eyes; metatarsomere I with some platellae. However the overall evidence from the molecular data, morphological observations (particularly the fused connective and aedeagus), and distribution support the placement of these genera in the tribe.

Cochlorhinini is potentially related to Koebeliini, Mukariini, Vartini or some Athysanini, but more data are needed to resolve its relationship to other tribes.

\section{Selected references}

Beamer (1940), Oman (1949), DeLong (1964).

\section{Included genera}

Allygianus Ball, 1936 placement nov. (transferred from Athysanini)

Allygiella Oman, 1949 placement nov. (transferred from Athysanini)

Ballana DeLong, 1936 placement nov. (transferred from Athysanini)

Calonia Beamer, 1940

Cochlorhinus Uhler, 1876 
Drionia Ball, 1915

Eulonus Oman, 1949

Gloridonus Ball, 1936 placement nov. (transferred from Athysanini)

Huleria Ball, 1902

Limbanus Oman, 1949 placement nov. (transferred from Athysanini)

Pasadenus Ball, 1936 placement nov. (transferred from Athysanini)

Penehuleria Beamer, 1934

Deltocephalini Dallas, 1870

Fig. 17

Type genus: Deltocephalus Burmeister, 1838.

\section{Diagnosis}

Deltocephalini are small to medium sized leafhoppers and are variable in color. They can be identified by the tapering or parallel-sided clypellus, narrow lorum, linear connective with anterior arms closely appressed, connective fused to the aedeagus, and first valvula dorsal sculpturing imbricate.

\section{Description}

HeAd. Head subequal to or wider than pronotum. Discal portion of crown glabrous with radial or longitudinal striae or shagreen. Anterior margin of head shagreen, rounded or angled to face. Frontoclypeus not tumid; texture shagreen. Clypellus parallel-sided or tapering apically; apex following or slightly surpassing normal curve of gena. Lorum distinctly narrower than clypellus near base. Antennal bases near middle or posteroventral (lower) corners of eyes. Antennae short, less than $1.5 \mathrm{x}$ width of head. Gena obtusely incised laterally; with fine erect seta beside laterofrontal suture. Antennal ledge absent. Ocelli present; close to eyes; on anterior margin of head.

THORAX. Pronotum lateral margin not carinate; lateral margin shorter than basal width of eye.

WiNGS. Forewing brachypterous to macropterous; appendix absent, reduced, or present and restricted to anal margin; with 3 anteapical cells; veins not raised; without reflexed costal veins; A1-A2 crossvein usually absent.

LEGS. Profemur with AM1 seta only; intercalary row with one row of five or more fine setae; row AV with short, stout setae. Protibia dorsal surface rounded, convex. Metafemur apex macrosetae $2+2+1$. Metatarsomere I not expanded apically; plantar setae simple, tapered.

Male genitalia. Valve articulated with pygofer or rarely fused to subgenital plates (Miradeltaphus, Yuanamia); lateral margin short, articulating with pygofer at a point. Pygofer basolateral membranous cleft present; macrosetae well differentiated into several rows. Subgenital plates free from each other; articulated with valve or rarely fused to each other (Miradeltaphus, Yuanamia); macrosetae uniseriate laterally. Style broadly bilobed basally, median anterior lobe pronounced. Basal processes of the aedeagus/connective absent or reduced or present, connected or articulated to connective or near base of aedeagus. Aedeagus with single shaft and gonopore. Connective anterior arms closely appressed anteriorly, linear-shaped; fused to aedeagus.

Female genitalia. Pygofer with numerous macrosetae. Ovipositor not protruding far beyond pygofer apex. First valvula convex or not strongly convex; dorsal sculpturing pattern imbricate (with overlapping scales); sculpturing reaching dorsal margin; without distinctly delimited ventroapical sculpturing. Second valvula broad, gradually tapered; without dorsal median tooth; dorsal teeth on apical $1 / 3$ or more; teeth small, regularly or irregularly shaped. 


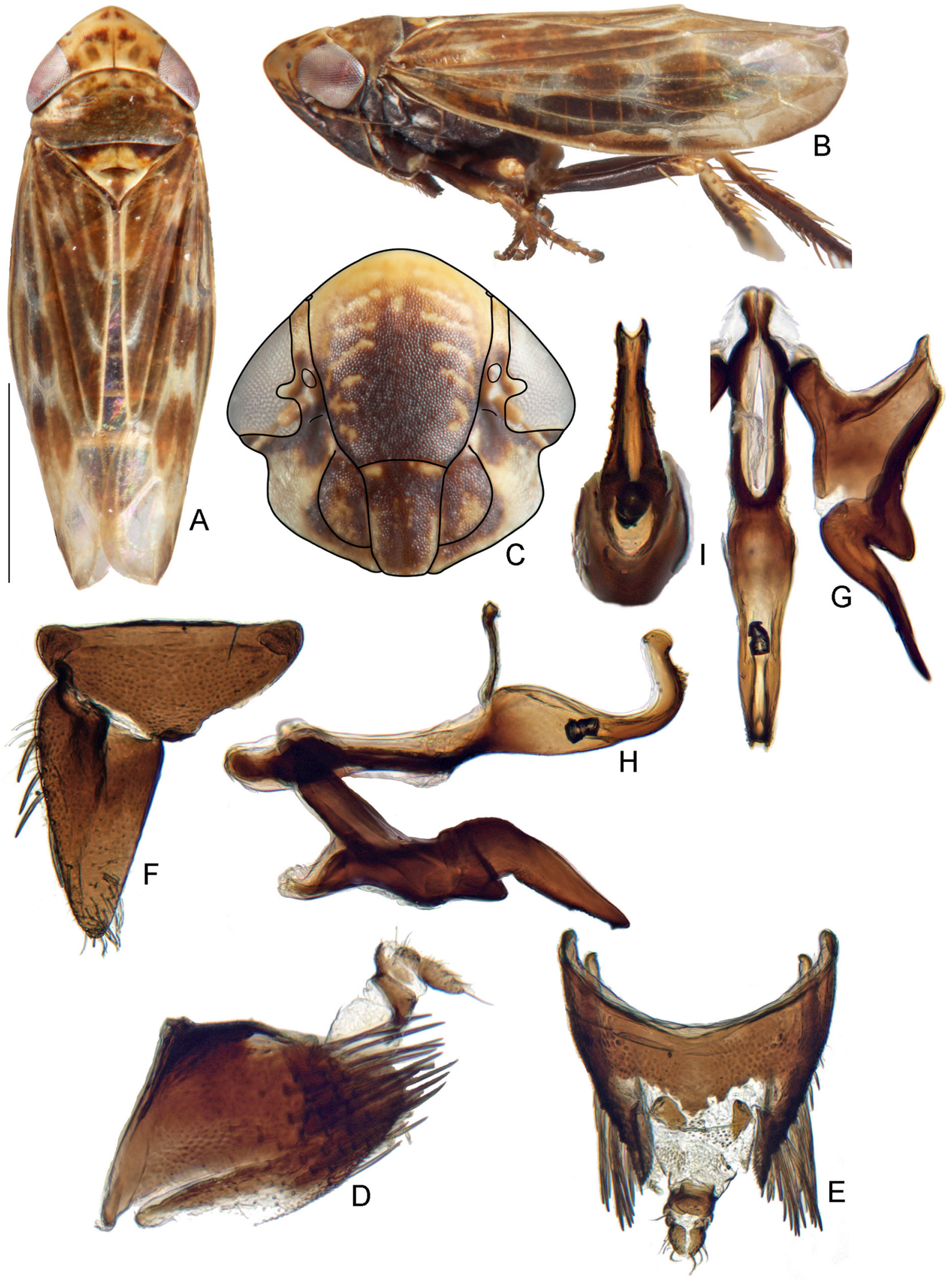

Fig. 17. Deltocephalini. Deltocephalus pulicaris (Fallén, 1806). A-I. Standard views (see Material \& Methods). G. Connective and style shown with aedeagus fused to connective. H. Aedeagus shown with connective and style. 


\section{Geography and ecology}

Distribution: cosmopolitan. Deltocephalini feed on grasses and sedges and are diverse and abundant in grassland ecosystems. Several species are economically important as vectors of disease agents. Graminella nigrifrons (Forbes, 1885), Maiestas dorsalis (Motschulsky, 1859) and Endria inimica (Say, 1830) are vectors of pathogens of maize, rice, and wheat, respectively.

\section{Remarks}

Deltocephalini contains 68 genera and 587 species. Morphological and molecular phylogenetic analyses to date suggest that it is monophyletic and closely related to Paralimnini, but further taxon sampling in these large tribes and more data are needed. The two tribes together appear to be related to Tetartostylini which also possesses the tapered clypellus and linear connective. Many genera and species of Deltocephalini are morphologically quite similar to each other, and some genera, e.g., Amplicephalus and Polyamia, are probably artificial. Revisions of many genera and identification keys, especially of the Neotropical fauna, are needed. As presently defined, the tribe encompasses the "Deltocephalus-group" of genera recognized by Fang et al. (1993, 1995) and Webb \& Viraktamath (2009), but is considerably narrower than the concepts of Deltocephalini employed by Oman (1949) and Linnavuori (1959).

\section{Selected references}

Oman (1949), Linnavuori (1959), Fang et al. (1993, 1995), Blocker et al. (1995), Dmitriev (2004b), Webb \& Viraktamath (2009).

\section{Included genera}

Afrosus Linnavuori, 1959

Alobaldia Emeljanov, 1972

Alodeltocephalus Evans, 1966

Amblysellus Sleesman, 1929

Amplicephalus DeLong, 1926

Arundanus DeLong, 1935

Bolarga Oman, 1938

Bolivaia Linnavuori \& DeLong, 1979

Cabrellus Emeljanov, 1964

Cabrulus Oman, 1949

Crumbana Oman, 1949

Cruziella Linnavuori \& DeLong, 1979

Ctenurellina McKamey, 2003

Daltonia Oman, 1949

Deltanus Oman, 1949

Deltazotus Kramer, 1971

Deltella Oman, 1949

Deltocephalus Burmeister, 1838

Destria Oman, 1949

Endria Oman, 1949

Fusanus Linnavuori, 1955

Graminella DeLong, 1936

Haldorus Oman, 1938

Heidinus Theron, 1988 placement nov. (previously unplaced in Deltocephalinae)

Horouta Knight, 1975

Kanorba Oman, 1938

Kansendria Kramer, 1971 
Limpica Cheng, 1980

Lonatura Osborn \& Ball, 1898

Lorellana DeLong \& Kolbe, 1975

Loreta Linnavuori, 1959

Lusitanocephalus Quartau, 1970

Maiestas Distant, 1917

Maricaona Caldwell, 1952

Matsumuratettix Metcalf, 1952

Mattogrossus Linnavuori, 1959

Mendozellus Linnavuori, 1959

Mexara Oman, 1949

Miradeltaphus Dash \& Viraktamath, 1995

Neodeltocephalus Linnavuori, 1959

Nullamia DeLong, 1970

Ochromelanus Stiller, 2011

Onura Oman, 1938

Paramesodes Ishihara, 1953

Parandanus Linnavuori \& DeLong, 1976

Parayuanamia Xing \& Li, 2011

Peitouellus Vilbaste, 1969

Picchuia Linnavuori \& DeLong, 1979

Planicephalus Linnavuori, 1954

Polyamia DeLong, 1926

Ragia Theron, 1973

Recilia Edwards, 1922

Reventazonia Linnavuori, 1959

Sanctanus Ball, 1932

Sanluisia Linnavuori, 1959

Spathifer Linnavuori, 1955

Tetramelasma Stiller, 2011

Theronus Stiller, 2009

Tideltellus Kramer, 1971

Toldoanus Linnavuori, 1954

Tumupasa Linnavuori, 1959

Unerus DeLong, 1936

Unoka Lawson, 1928

Vicosa Linnavuori \& DeLong, 1978

Wakaya Linnavuori, 1960

Warlucephala Fletcher, 2006

Wyushinamia Zhang \& Duan, 2006

Yuanamia Zhang \& Duan, 2006

Dorycephalini Oman, 1943

Fig. 18

Type genus: Dorycephalus Kouchakewitch, 1866.

\section{Diagnosis}

Dorycephalini are medium sized, elongate, dorsoventrally flattened, greenish or ivory to brownish leafhoppers. They can be identified by the strongly produced, spatulate, and flattened head with anterior 
margin foliaceous, long pronotum, raised forewing veins, forewing with two anteapical cells, metafemur apex macrosetae $2+0$, and hind tibia distinctly bent apically. Eupelicini and some Hecalini (particularly Attenuipyga and Neoslossonia which were previously placed in Dorycephalini) share some of these characters, but Dorycephalini can be distinguished from Eupelicini by the crown not encroaching onto eyes as in Eupelicina, head wider and more spatulate-shaped than Paradorydiina, and valve not fused to the pygofer and style not gracile as in Paradorydiina. From Attenuipyga and Neoslossonia (Hecalini), Dorycephalini can be distinguished by the forewing veins A1 and A2 separate throughout their length, male pygofer lobate apically and not distinctly pointed posterodorsally, style apophysis slightly expanded, rounded apically, aedeagus without processes, female first valvula without distinctly delimited ventroapical sculpturing, second valvulae not humpbacked dorsally, and second valvulae with distinct teeth.

\section{Description}

HEAD. Head subequal to or wider than pronotum; elongate; spatulate. Discal portion of crown rugose. Anterior margin of head foliaceous. Frontoclypeus tumid; texture shagreen. Clypellus parallel-sided or tapering apically; apex following or slightly surpassing normal curve of gena. Lorum distinctly narrower than clypellus near base. Antennal bases near upper or anterodorsal corners of eyes. Antennae short, less than $1.5 \mathrm{x}$ width of head. Gena strongly incised laterally (nearly forming right angle); without fine erect seta beside laterofrontal suture. Antennal ledges absent. Ocelli present; somewhat distant from eyes; on crown.

Thorax. Pronotum lateral margin carinate; lateral margin as long as or longer than basal width of eye.

Wings. Forewing submacropterous to brachypterous; appendix absent or reduced; with 2 anteapical cells; veins distinctly raised or carinate; without reflexed costal veins; A1-A2 crossvein absent; A1 and A2 separate from each other throughout their length; apical venation not highly reticulate.

LEGS. Profemur with AM1 absent or reduced; intercalary row reduced or absent; row AV with relatively long macrosetae. Protibia dorsal surface rounded, convex. Metafemur apex macrosetae $2+0$. Metatarsomere I expanded apically; plantar surface with one or more platellae.

Male genitalia. Apodemes of sternite I long and wide; those of sternite II not well-developed. Valve articulated with pygofer; with short point of articulation with pygofer. Pygofer dorsoapical margin incised to near mid-length; basolateral membranous cleft present; macrosetae absent or reduced ( $\leq$ two rows). Subgenital plates free from each other; articulated with valve; without macrosetae. Style broadly bilobed basally, median anterior lobe pronounced. Basal processes of the aedeagus/connective absent. Aedeagus without basal hinge; with a single shaft and gonopore. Connective anterior arms somewhat divergent, $\mathbf{Y}$ - or $\mathbf{U}$-shaped; articulated with aedeagus.

Female genitalia. Pygofer with macrosetae reduced or absent. Ovipositor not protruding far beyond pygofer apex. First valvula not strongly convex; dorsal sculpturing pattern granulose or maculose; sculpturing submarginal; without distinctly delimited ventroapical sculpturing. Second valvula broad, gradually tapered; without dorsal median tooth; teeth on apical $1 / 3$ or more; teeth small, regularly or irregularly shaped.

\section{Geography and ecology}

Distribution: Palearctic. The two known species feed on grasses. Their elongate, flattened shape appears to be an adaptation for crypsis and closely appressing against grass blades. 


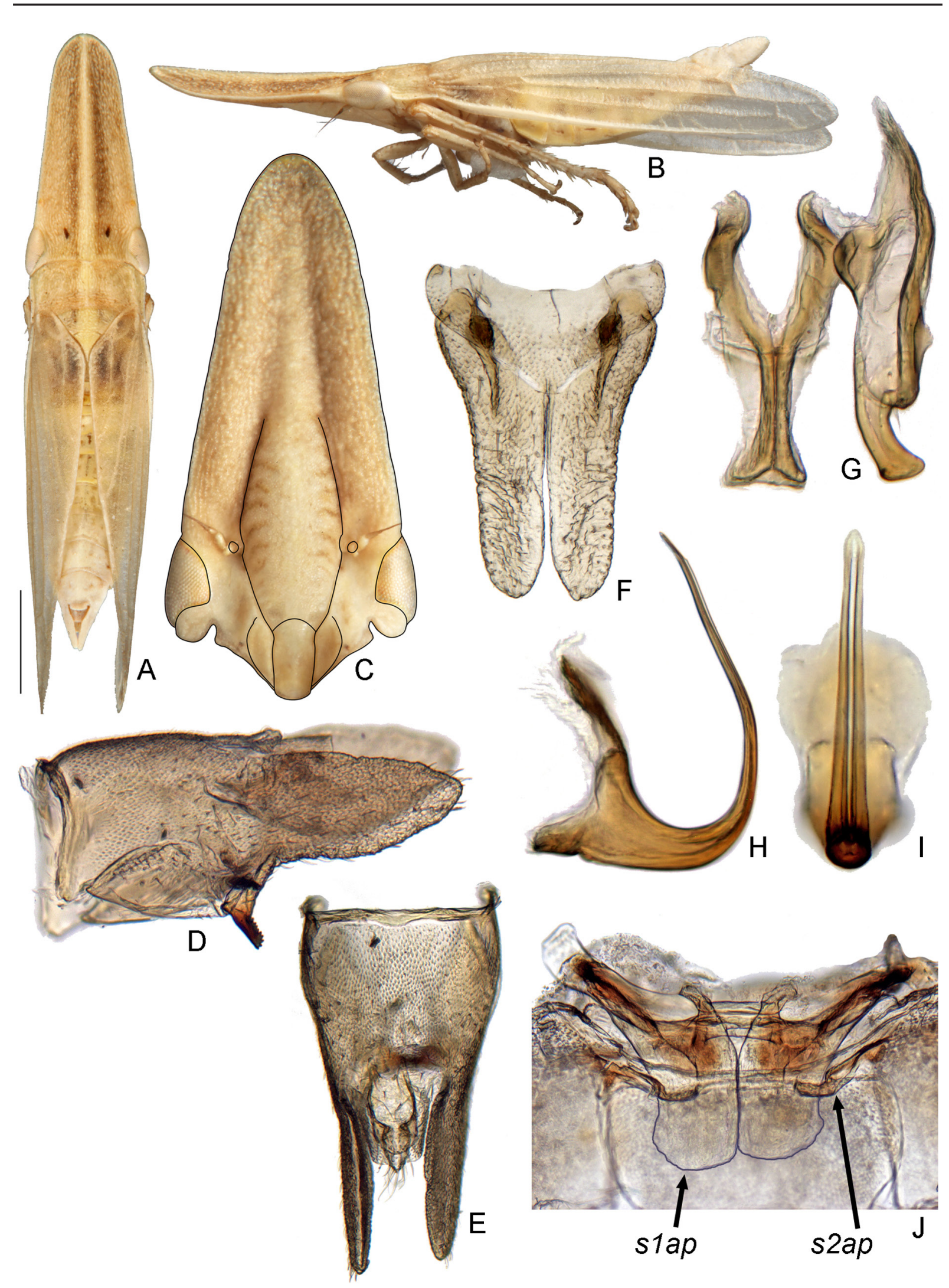

Fig. 18. Dorycephalini. Dorycephalus baeri Kouchakewitch, 1866. A-I. Standard views (see Material \& Methods). J. Ventral view of abdominal sternites I-III, showing large apodemes of sternite I (slap) and apodemes of sternite II (s2ap). 


\section{Remarks}

Dorycephalini as circumscribed here contains only the type genus and its 2 species. Dorycephalus was included in molecular phylogenetic analyses here for the first time. It was added to the data set only after branch support analyses were already completed so it is not present in the trees figured with branch support but is included in Fig. 6. In ML analyses of the molecular data only (28S + H3) Dorycephalus was recovered in the grass-specialist clade including Chiasmini, Eupelicini, Stenometopiini, and some others, and was quite distant from the long branch uniting Hecalus and Attenuipyga. Based on this result and because it does not share the characters which unite Attenuipyga, Neoslossonia, and Hecalini (see Diagnosis of Hecalini below), Dorycephalini is retained as a monotypic tribe here, and Attenuipgyga and Neoslossonia are placed in Hecalini.

\section{Selected references}

Oman (1985a), Dmitriev (2001).

\section{Included genera}

Dorycephalus Kouchakewitch, 1866

\section{Drabescini Ishihara, 1953}

Figs 19, 20

Type genus: Drabescus Stål, 1870.

= Paraboloponini Ishihara, 1953.

= Bhatiini Linnavuori \& Al-Ne'Amy, 1983.

\section{Diagnosis}

Drabescini are medium sized to large leafhoppers, variable in color and shape. They can be identified by the antennae situated near the upper part of the face, antennal pits large and often encroaching onto the frontoclypeus, anterior margin of the head glabrous, irregularly textured, or with one to many carinae or striae, long antennae, nymph often with apical process on the head and male pygofer with a pair of long apical appendages widely separated at the base.

\section{Description}

HEAD. Head subequal to or wider than pronotum. Discal portion of crown glabrous with radial or longitudinal striae, or shagreen. Anterior margin of head glabrous, irregularly textured, or with one to many transverse carinae. Frontoclypeus not tumid; texture shagreen, glabrous, or striate (Drabescina). Clypellus widening apically; apex following or slightly surpassing normal curve of gena. Lorum subequal to or wider than clypellus near base. Antennal bases near upper or anterodorsal corners of eyes; antennal pits usually large, encroaching on frontoclypeus. Antennae long, as long as width of head or longer. Gena obtusely incised laterally; with fine erect seta beside laterofrontal suture. Antennal ledge weakly developed (carinate or weakly carinate) or strongly developed, with a definite ledge (Drabescina). Ocelli present; close to or distant from eyes; on anterior margin of head.

Thorax. Pronotum exceeding or not exceeding eyes anteriorly; lateral margin carinate or not; lateral margin shorter than basal width of eye.

WiNGS. Forewing macropterous; appendix restricted to anal margin or large, extending around wing apex (Drabescina); with 3 anteapical cells; veins not raised; with or without reflexed costal veins; A1-A2 crossvein absent or present. 
LEgs. Profemur with AM1 seta only; intercalary row with one row of five or more fine setae, or not in one row, scattered; row AV with thin hair-like setae, without setae, or rarely with short or long macrosetae. Protibia dorsal surface rounded, convex (Paraboloponina) or dorsal surface flat, sharply carinate along $\mathrm{AD}$ and PD margins (Drabescina). Metafemur apex macrosetae $2+1,2+1+1$, or $2+2+1$. Metatarsomere I not expanded apically; plantar setae simple, tapered.

Male Genitalia. Valve articulated with pygofer; lateral margin short, articulating with pygofer at a point. Pygofer basolateral membranous cleft present; macrosetae absent or reduced ( $\leq$ two rows) or present, well differentiated into several rows. Subgenital plates free from each other; articulated with valve; usually without macrosetae. Style broadly bilobed basally, median anterior lobe pronounced. Basal processes of the aedeagus/connective absent or reduced or present, connected or articulated to connective or near base of aedeagus. Aedeagus with single shaft and gonopore. Connective anterior arms somewhat divergent, $\mathbf{Y}$ - or $\mathbf{U}$-shaped; articulated with aedeagus.

Female Genitalia. Pygofer with numerous macrosetae. Ovipositor not protruding far beyond pygofer apex. First valvula convex or not strongly convex; dorsal sculpturing pattern strigate; sculpturing reaching dorsal margin; without distinctly delimited ventroapical sculpturing. Second valvula broad, gradually tapered; with or without dorsal median tooth; dorsal teeth on apical 1/3 or more or restricted to apical $1 / 4$ or less; teeth large, regularly shaped or small, regularly or irregularly shaped.

\section{Geography and ecology}

Distribution: Palearctic, Afrotropical, Oriental and Australian regions. Drabescini are often found on woody hosts in Old World tropical or deciduous forests, and are often collected at lights.

\section{Remarks}

Drabescini contains 38 genera and 187 valid species. Dmitriev (2004a) synonymized Drabescini and Paraboloponini based on shared characters of the nymph, and its monophyly was supported in phylogenetic analyses. Here, we accept the synonymy by Dmitriev (2004a) but continue to recognize separate subtribes following the circumscription of the two groups by Zhang \& Webb (1996) due to the morphological differences of the adults that distinguish them. The phylogenetic analyses here suggest that the tribe is closely related to Scaphoideini, with which it shares long antennae and profemur row AV with thin hair-like setae or without setae. Our analyses provided no evidence of a close relationship between Drabescini and Selenocephalini, which some previous authors (e.g., Linnavuori \& Al-Ne'amy 1983; Zhang \& Webb 1996) placed together in a separate subfamily, Selenocephalinae.

\section{Selected references}

Linnavuori (1978a, b), Webb (1981), Zhang \& Webb (1996), Viraktamath (1998), Dmitriev (2002, 2004a).

Included subtribes:

\section{Drabescina Ishihara, 1953}

Fig. 19

Type genus: Drabescus Stål, 1870.

\section{Diagnosis}

Drabescina are large, robust, black, grey, or fuscous leafhoppers. They can be distinguished from Paraboloponina by the very strong antennal ledges, antennae usually somewhat shorter- 1.0 to $1.5 \mathrm{x}$ 
width of head, rugose or striate texture of the frontoclypeus, protibia flattened and expanded dorsally and with dorsal margins sharply carinate, and forewing appendix large and extending to the wing apex.

\section{Description}

HeAd. Head subequal to or wider than pronotum. Discal portion of crown glabrous with radial or longitudinal striae. Anterior margin of head glabrous, irregularly textured, or with numerous transverse striations or carinae. Frontoclypeus not tumid; texture glabrous or striate. Clypellus widening apically; apex following or slightly surpassing normal curve of gena. Lorum subequal to or wider than clypellus near base. Antennal bases near upper or anterodorsal corners of eyes. Antennae long, as long as width of head or longer. Gena obtusely incised laterally; with fine erect seta beside laterofrontal suture. Antennal ledges strongly developed (with a definite ledge). Ocelli present; distant from eyes; on anterior margin of head.

THORAx. Pronotum lateral margin carinate; lateral margin shorter than basal width of eye.

WINGS. Forewing macropterous; appendix large, extending to wing apex; with 3 anteapical cells; veins not raised; without reflexed costal veins; A1-A2 crossvein absent or present; apical venation not highly reticulate.

LEGs. Profemur with AM1 seta only; intercalary row setae not in one row or scattered; row AV with thin, hair-like setae or without setae. Protibia dorsal surface flat; sharply carinate along AD and PD margins. Metafemur apex macrosetae 2+1. Metatarsomere I not expanded apically; plantar setae simple, tapered.

MaLe Genitalia. Valve articulated with pygofer; with short point of articulation with pygofer. Pygofer basolateral membranous cleft present; macrosetae absent or reduced ( $\leq$ two rows) or well differentiated into several rows. Subgenital plates free from each other; articulated with valve; without macrosetae. Style broadly bilobed basally, median anterior lobe pronounced; apophysis digitate, lobate, or incrassate with distinct strigate to reticulate texturing. Aedeagus usually with pair of fused basal processes; without basal hinge; with a single shaft and gonopore. Connective anterior arms somewhat divergent, $\mathbf{Y}$-shaped; articulated with aedeagus.

Female genitalia. Pygofer with numerous macrosetae. Ovipositor not protruding far beyond pygofer apex. First valvula convex; dorsal sculpturing pattern strigate; sculpturing reaching dorsal margin; without distinctly delimited ventroapical sculpturing. Second valvula abruptly broadened medially or subapically or broad, gradually tapered; with dorsal median tooth; teeth on apical $1 / 3$ or more or teeth restricted to apical 1/4 or less; teeth large, regularly shaped or teeth small, regularly or irregularly shaped.

\section{Geography and ecology}

Distribution: Palearctic, Afrotropical and Oriental regions. Species of Drabescus have been collected at lights and sweeping woody forest vegetation.

\section{Remarks}

Drabescina contains 2 genera and 55 species.

\section{Included genera}

Drabescus Stål, 1870

Rengatella Zhang \& Webb, 1996 


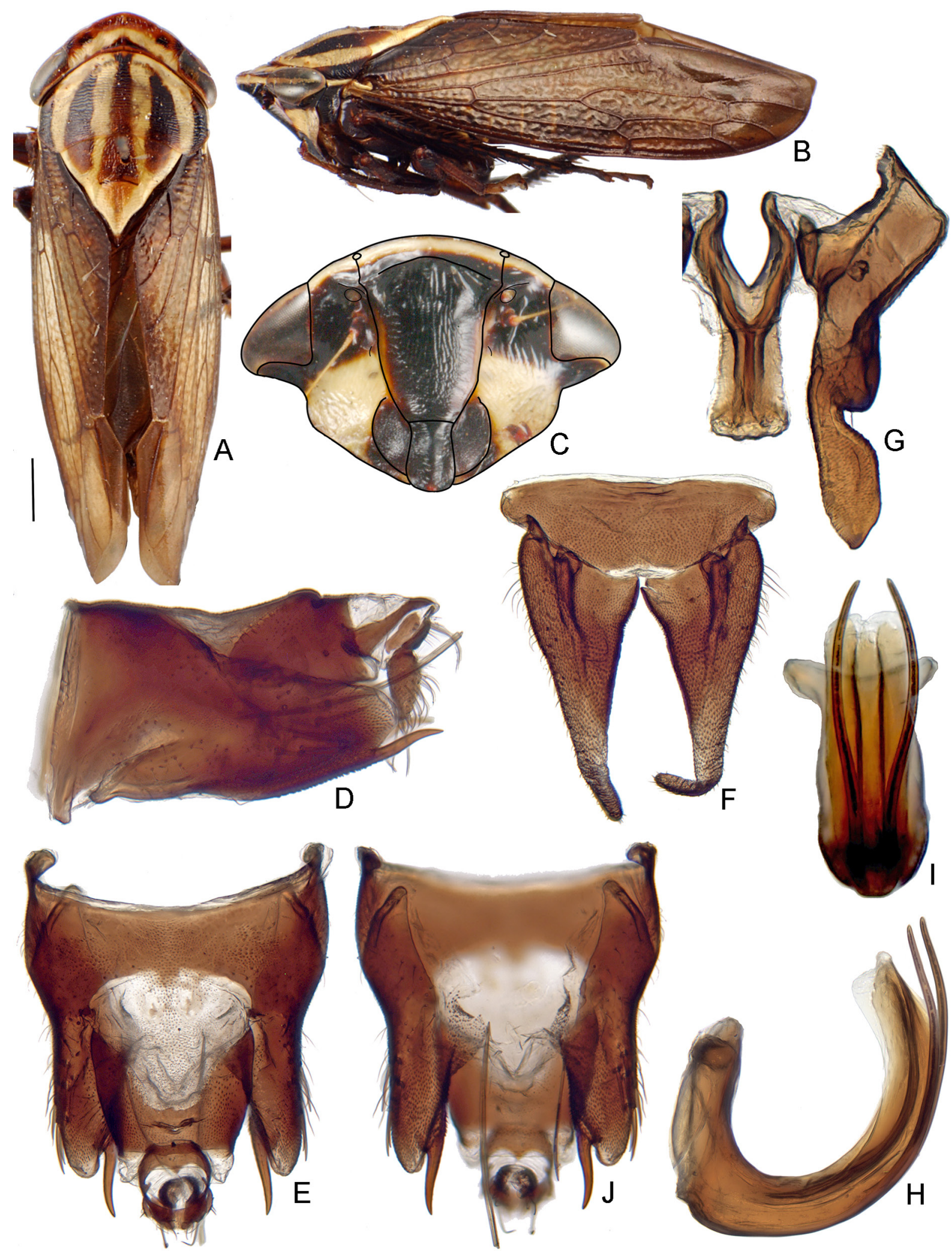

Fig. 19. Drabescini (Drabescina). Drabescus zhangi sp. nov. A-I. Standard views (see Material \& Methods). J. Ventral view of $\widehat{\partial}$ pygofer. 
Paraboloponina Ishihara, 1953

Fig. 20

Type genus: Parabolopona Matsumura, 1912.

\section{Diagnosis}

Paraboloponina are medium sized to large leafhoppers, and are variable in shape and color. They can be distinguished from Drabescina by the antennal ledges which are weak or absent, antennae usually longer, $1.5 \mathrm{x}$ width of head or longer, texture of the frontoclypeus shagreen, protibia rounded dorsally or rarely somewhat flattened, and forewing appendix not especially large.

\section{Description}

HEAD. Head subequal to or wider than pronotum. Discal portion of crown glabrous with radial or longitudinal striae, or shagreen. Anterior margin of head glabrous, irregularly textured, foliaceous, with numerous transverse striations, with 2 or 3 parallel carinae, or with numerous carinae. Frontoclypeus not tumid; texture shagreen or striate. Clypellus widening apically, apex following or slightly surpassing normal curve of gena. Lorum subequal to or wider than clypellus near base. Antennal bases near upper or anterodorsal corners of eyes. Antennal pits often very large and encroaching onto frontoclypeus. Antennae long, $1.5 \mathrm{x}$ width of head or longer. Gena not incised or obtusely incised laterally; with fine erect seta beside laterofrontal suture. Antennal ledges absent or weakly developed (carinate or weakly carinate). Ocelli present; close to or distant from eyes; on anterior margin of head.

THORAX. Pronotum lateral margin carinate; lateral margin shorter than basal width of eye.

WINGS. Forewings macropterous; appendix restricted to anal margin; with 3 anteapical cells; veins not raised; without or with reflexed costal veins; A1-A2 crossvein absent or present; apical venation not highly reticulate.

Legs. Profemur with AM1 seta only; intercalary row with one row of five or more fine setae; row AV with thin, hair-like setae or without setae, or rarely with short, stout setae. Protibia dorsal surface rounded, convex. Mesofemur row AV sometimes with several long macrosetae. Metafemur apex macrosetae $2+1$ or $2+1+1$ or $2+2+1$. Metatarsomere I not expanded apically, plantar setae simple, tapered.

Male Genitalia. Valve articulated with pygofer; with short point of articulation with pygofer. Pygofer basolateral membranous cleft present; macrosetae absent or reduced ( $\leq$ two rows) or well differentiated into several rows. Subgenital plates free from each other; articulated with valve; without macrosetae or with macrosetae scattered, irregularly arranged or uniseriate laterally. Style broadly bilobed basally, median anterior lobe pronounced. Basal processes of the aedeagus/connective absent or reduced or present, connected or articulated to connective or near base of aedeagus. Aedeagus without basal hinge; with a single shaft and gonopore. Connective anterior arms somewhat divergent, $\mathbf{Y}$ - or $\mathbf{U}$-shaped; articulated with aedeagus.

Female genitalia. Pygofer with numerous macrosetae. Ovipositor not protruding far beyond pygofer apex. First valvula convex; dorsal sculpturing pattern strigate, concatenate, or reticulate; sculpturing reaching dorsal margin; without distinctly delimited ventroapical sculpturing. Second valvula abruptly broadened medially or subapically or gradually broadened medially or subapically; with or without dorsal median tooth; teeth on apical $1 / 3$ or more or restricted to apical $1 / 4$ or less; teeth small, regularly or irregularly shaped. 


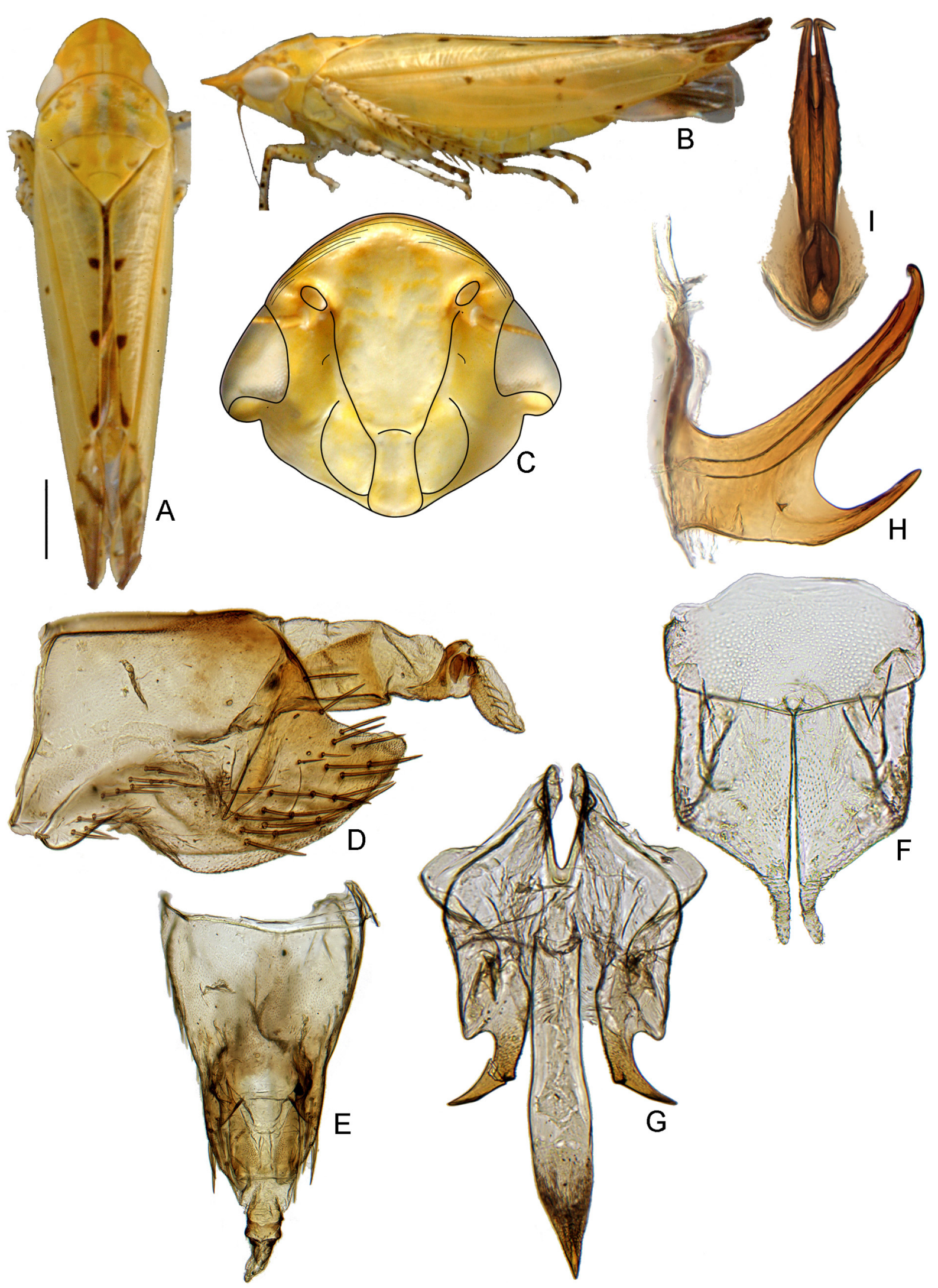

Fig. 20. Drabescini (Paraboloponina). Parabolopona webbi sp. nov. A-I. Standard views (see Material $\&$ Methods). G. Connective shown with fused process. 


\section{Geography and ecology}

Distribution: Palearctic, Afrotropical, Oriental and Australian regions.

\section{Remarks}

Paraboloponina contains 36 genera and 132 species.

\section{Included genera}

Athysanopsis Matsumura, 1914

Bhatia Distant, 1908

Canopyana Viraktamath \& Srinivasa, 2006

Carvaka Distant, 1918

Divus Distant, 1908

Drabescoides Kwon \& Lee, 1979

Dryadomorpha Kirkaldy, 1906

Eminea Seven, 1997

Favintiga Webb, 1981

Halimunella Kamitani, 2012

Hybrasil Kirkaldy, 1907

Indokutara Viraktamath, 1998

Isaca Walker, 1857

Jamitettix Matsumura, 1940

Karoseefa Webb, 1981

Kotabala Viraktamath, 1998

Kutara Distant, 1908

Megabyzus Distant, 1908

Mysolis Kirkaldy, 1904

Nakula Distant, 1918

Nirvanguina Zhang \& Webb, 1996

Oceanopona Linnavuori, 1960

Odmiella Linnavuori, 1978

Odzalana Linnavuori, 1969

Omanella Merino, 1936

Parabolopona Matsumura, 1912

Parohinka Webb, 1981

Rhutelorbus Webb, 1981

Roxasella Merino, 1936

Roxasellana Zhang \& Zhang, 1998

Sombakidia Zhang \& Webb, 1996

Stenomiella Evans, 1955

Tengatka Zhang \& Webb, 1996

Tenompoella Zhang \& Webb, 1996

Waigara Zhang \& Webb, 1996

Welmaya Zhang \& Webb, 1996

Drakensbergenini Linnavuori, 1979

Fig. 21

Type genus: Drakensbergena Linnavuori, 1961. 


\section{Diagnosis}

Drakensbergenini are small to large, ivory, ochraceous, to dark brown brachypterous leafhoppers, often with a dark brown median stripe from apex of crown to abdomen. They can be identified by the produced head, ocelli on the crown, tumid frontoclypeus, strongly tapering clypellus, and brachypterous forewings.

\section{Description}

HeAd. Head subequal to or wider than pronotum; somewhat to strongly produced. Discal portion of crown shagreen. Anterior margin of head shagreen or foliaceous; often shagreen and rounded to face laterally and foliaceous apically. Frontoclypeus tumid; texture shagreen. Clypellus strongly tapering apically; apex following or slightly surpassing normal curve of gena. Lorum distinctly narrower than

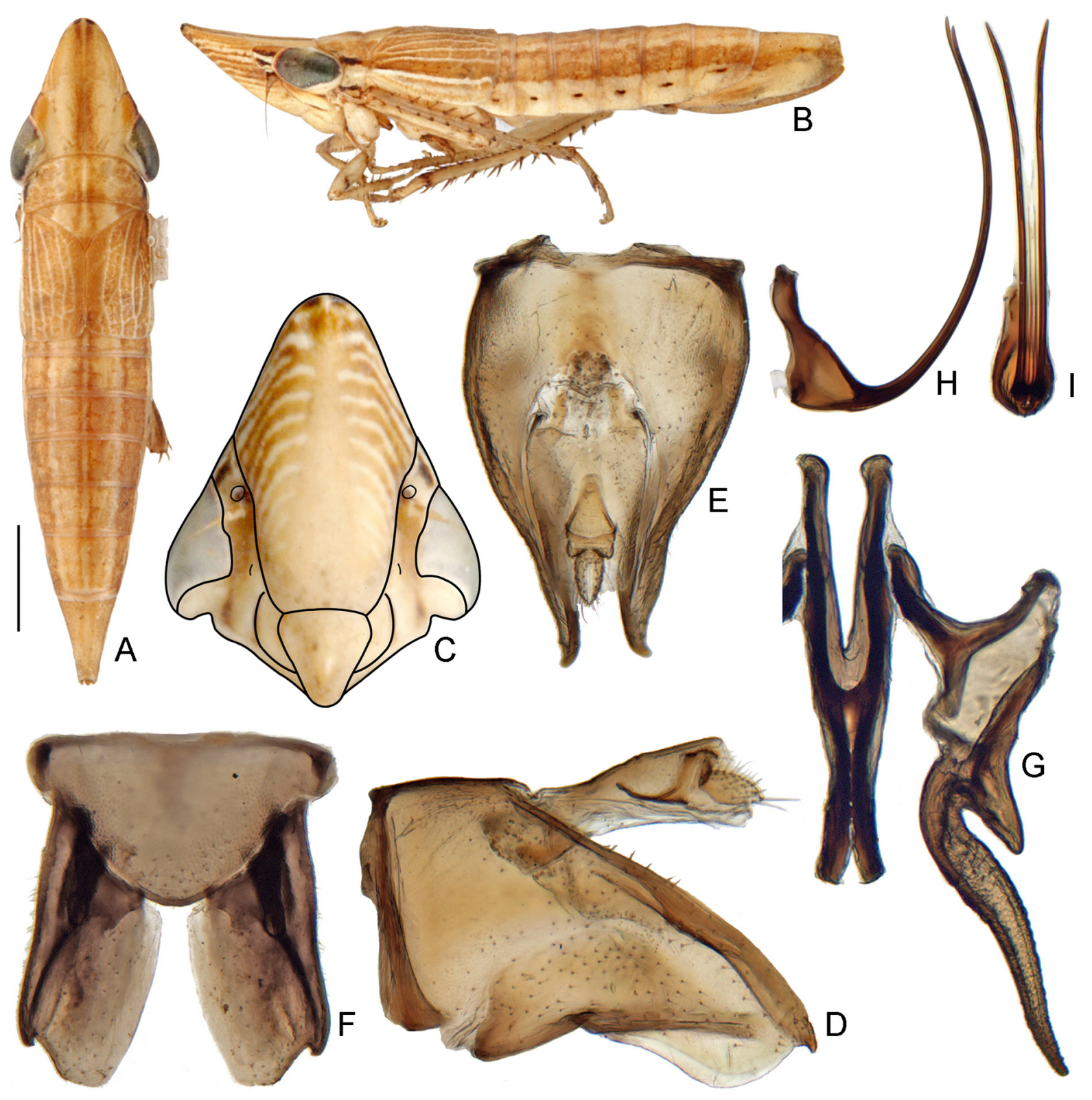

Fig. 21. Drakensbergenini. A-B. Drakensbergena deorsuspina Stiller, 2009. C. Drakensbergena sp. D-I. D. phaeogramma Stiller, 2009. A-I. Standard views (see Material \& Methods). 
clypellus near base. Antennal bases near upper or anterodorsal corners of eyes. Antennae short, less than $1.5 \mathrm{x}$ width of head or long, $1.5 \mathrm{x}$ width of head or longer. Gena strongly incised laterally (nearly forming right angle); with fine erect seta beside laterofrontal suture. Antennal ledge weakly developed (carinate or weakly carinate). Ocelli reduced or present; distant from eyes; on crown.

THORAX. Pronotum lateral margin carinate or rarely ecarinate; lateral margin shorter than basal width of eye.

WINGs. Forewing brachypterous; coriaceous; wings usually fused; veins obscure.

LEGS. Profemur with AM1 absent or reduced; intercalary row reduced or absent; row AV with relatively long macrosetae. Protibia dorsal surface rounded, convex. Metafemur apex macrosetae $2+1$ or $2+1+1$. Metatarsomere I expanded apically; plantar setae simple, tapered.

Male Genitalia. Valve articulated with pygofer; lateral margin short, articulating with pygofer at a point. Pygofer basolateral membranous cleft present; macrosetae absent or reduced ( $\leq$ two rows). Subgenital plates free from each other; articulated with valve; without macrosetae. Style broadly bilobed basally, median anterior lobe pronounced. Basal processes of the aedeagus/connective absent or reduced. Aedeagus with single shaft and gonopore; apex bifid. Connective anterior arms somewhat divergent, Y-shaped; stem often appearing to be formed by two longitudinal bars; articulated with aedeagus. Segment X long; sclerotized dorsally and laterally.

Female Genitalia. Pygofer with macrosetae reduced or absent. Ovipositor not protruding far beyond pygofer apex. First valvula not strongly convex; dorsal sculpturing pattern concatenate, reticulate, granulose, or maculose; sculpturing reaching dorsal margin or submarginal; without distinctly delimited ventroapical sculpturing. Second valvula abruptly broadened medially or subapically or broad, gradually tapered; without dorsal median tooth; dorsal teeth on apical $1 / 3$ or more; teeth small, regularly or irregularly shaped. Third valvula with two irregular setal rows along entire ventral margin.

\section{Geography and ecology}

Distribution: Afrotropical region (South Africa and Lesotho). Drakensbergena spp. feed on grasses in the high elevation grassland biome of southern Africa.

\section{Remarks}

Drakensbergenini contains the type genus and 18 species. This group was considered a separate subfamily by Linnavuori (1979a) but placed as a tribe of Deltocephalinae by Zahniser \& Dietrich (2010). Stiller (2009) described 16 new species of the genus. Phylogenetic analyses and morphological characters (produced head, profemur intercalary row reduced, AM1 seta reduced or absent, AV setae relatively long, metafemur apical setae $2+1+1$ or $2+1$ ) suggest that this tribe is related to Eupelicini.

\section{Selected references}

Linnavuori (1979a), Stiller (2009).

\section{Included genera}

Drakensbergena Linnavuori, 1961

Eupelicini Sahlberg, 1871

Figs 22, 23

Type genus: Eupelix Germar, 1821. 


\section{Diagnosis}

Eupelicini are medium sized to large ivory, ochraceous, green, or brownish leafhoppers. They can be identified by the strongly produced or spatulate head, gena without fine erect seta beside laterofrontal suture, genal margins strongly emarginate $\left(\sim 90^{\circ}\right.$ angle $)$, forewing veins raised or carinate, metafemur apical setae $2+0$, metatarsomere I expanded apically, male pygofer macrosetae absent or reduced, subgenital plates without macrosetae, and aedeagus not hinged basally.

\section{Description}

HEAD. Head subequal to or wider than pronotum; somewhat to very strongly produced anteriorly. Discal portion of crown shagreen, granulose, or punctate. Anterior margin of head foliaceous. Frontoclypeus elongate; not tumid; texture shagreen or punctate. Clypellus parallel-sided, tapering apically, or widening apically; apex following or slightly surpassing normal curve of gena; notched apically. Lorum subequal to or wider than clypellus near base. Antennal bases near upper or anterodorsal corners of eyes. Gena strongly incised laterally (nearly forming right angle); without fine erect seta beside laterofrontal suture. Antennae short, less than $1.5 \mathrm{x}$ width of head. Antennal ledge absent or weakly developed (carinate or weakly carinate). Antennal bases near upper or anterodorsal corners of eyes. Ocelli present; close to or distant from eyes; on anterior margin of head or on crown.

Thorax. Pronotum usually with median longitudinal carina and sublateral keels or carinae; lateral margin carinate or not; lateral margin as long as or longer than basal width of eye.

WiNGS. Forewing macropterous or submacropterous; appendix absent or reduced (Paradorydiina) or appendix large, extending around wing apex (Eupelicina); with 3 anteapical cells; veins distinctly raised or carinate; without reflexed costal veins; A1-A2 crossvein absent.

LEgs. Profemur with AM1 absent or reduced; intercalary row reduced or absent; row AV without setae or with relatively long macrosetae. Protibia dorsal surface flat, $\mathrm{AD}$ and $\mathrm{PD}$ margins at $\sim 90^{\circ}$ angles but not carinate. Metafemur apex macrosetae $2+0$. Metatibia row AV macrosetae restricted to apical $1 / 2$ or less. Metatibia not strongly compressed laterally, more or less square shaped in cross section; distinctly bent apically in dorsal view. Metatarsomere I expanded apically; plantar setae simple, tapered.

Male genitalia. Valve articulated with pygofer or fused to pygofer (Paradorydiina), if articulated then lateral margin short, articulating with pygofer at a point. Pygofer basolateral membranous cleft present (Eupelicina) or absent (Paradorydiina); macrosetae absent or reduced ( $\leq$ two rows). Subgenital plates free from each other; articulated with or fused to valve; without macrosetae. Style broadly bilobed basally, median anterior lobe pronounced. Basal processes of the aedeagus/connective absent or reduced. Aedeagus with single shaft and gonopore. Connective anterior arms somewhat divergent, Y-or V-shaped; articulated with aedeagus.

Female Genitalia. Pygofer with macrosetae reduced or absent. Ovipositor protruding (Paradorydiina) or not protruding (Eupelicina) far beyond pygofer apex. First valvula not strongly convex; dorsal sculpturing pattern reticulate, granulose, maculose, or imbricate (with overlapping scales); sculpturing reaching dorsal margin or submarginal; with (Paradorydiina) or without (Eupelicina) distinctly delimited ventroapical sculptured area. Second valvula broad, gradually tapered or gradually broadened medially or subapically; with or without dorsal median tooth; dorsal teeth on apical 1/3 or more if present; teeth small, regularly or irregularly shaped (Eupelicina) or absent (Paradorydiina).

\section{Geography and ecology}

Distribution: Palearctic, Afrotropical, Oriental and Australian regions. All members are grass feeders. 


\section{Remarks}

Eupelicini contains 7 genera and 64 species. Listrophorina is removed from the tribe here and synonymized with Chiasmini, and Dorycephalini is considered a separate tribe following Zahniser \& Dietrich (2010). Eupelicini is supported as a monophyletic group in phylogenetic analyses and by shared morphological characters. It is related to other grass-feeding tribes of Deltocephalinae including Drakensbergenini, Chiasmini, and Stenometopiini.

\section{Selected references}

Evans (1966), Morrison (1973), Linnavuori (1979a, b), Viraktamath \& Viraktamath (1989), Dmitriev (2001), D’Urso (1992).

Included subtribes:

Eupelicina Sahlberg, 1871

Fig. 22

Type genus: Eupelix Germar, 1821.

\section{Diagnosis}

Eupelicina are medium sized to large, whitish leafhoppers. They can be distinguished from Paradorydiina by the crown partly extending over the eyes anteriorly, the dorsal position of the ocelli, lateral margin of the pronotum carinate, frontoclypeus with a median carina anteriorly, forewing appendix large and extending around the wing apex, forewing marginal vein complete, hind wing marginal vein complete, male valve not fused to the pygofer, style more robust, ovipositor not extending far beyond the pygofer apex, first valvula dorsal sculpturing pattern reticulate or imbricate, second valvula with a dorsal median tooth and with dorsal teeth on the apical $1 / 3$.

\section{Description}

HEAD. Head subequal to or wider than pronotum. Discal portion of crown shagreen. Anterior margin of head foliaceous. Frontoclypeus not tumid; texture shagreen; with median longitudinal carina toward apex. Clypellus parallel-sided or tapering apically; apex following or slightly surpassing normal curve of gena. Lorum subequal to or wider than clypellus near base. Antennal bases near upper or anterodorsal corners of eyes. Antennae short, less than $1.5 \mathrm{x}$ width of head. Gena strongly incised laterally (nearly forming right angle); without fine erect seta beside laterofrontal suture. Antennal ledges absent. Ocelli present; distant from eyes; on crown.

THORAX. Pronotum lateral margin carinate; lateral margin as long as or longer than basal width of eye.

WINGS. Forewing macropterous or submacropterous; appendix large, extending around wing apex; with 3 anteapical cells; veins distinctly raised or carinate; without reflexed costal veins; A1-A2 crossvein absent; apical venation not highly reticulate.

Legs. Profemur with AM1 absent or reduced; intercalary row reduced or absent; row AV with relatively long macrosetae. Protibia dorsal surface flat, $\mathrm{AD}$ and $\mathrm{PD}$ margins at $\sim 90^{\circ}$ angles but not carinate. Metafemur apex macrosetae 2+0. Metatarsomere I expanded apically; plantar setae in adult all simple.

MaLe Genitalia. Valve articulated with pygofer, with short point of articulation with pygofer. Pygofer basolateral membranous cleft present; macrosetae absent or reduced ( $\leq$ two rows). Subgenital plates free from each other; articulated with valve; without macrosetae. Style broadly bilobed basally; median anterior lobe pronounced. Basal processes of the aedeagus/connective absent. Aedeagus without basal 
ZAHNISER J.N. \& DIETRICH C.H., A review of the tribes of Deltocephalinae (Cicadellidae)

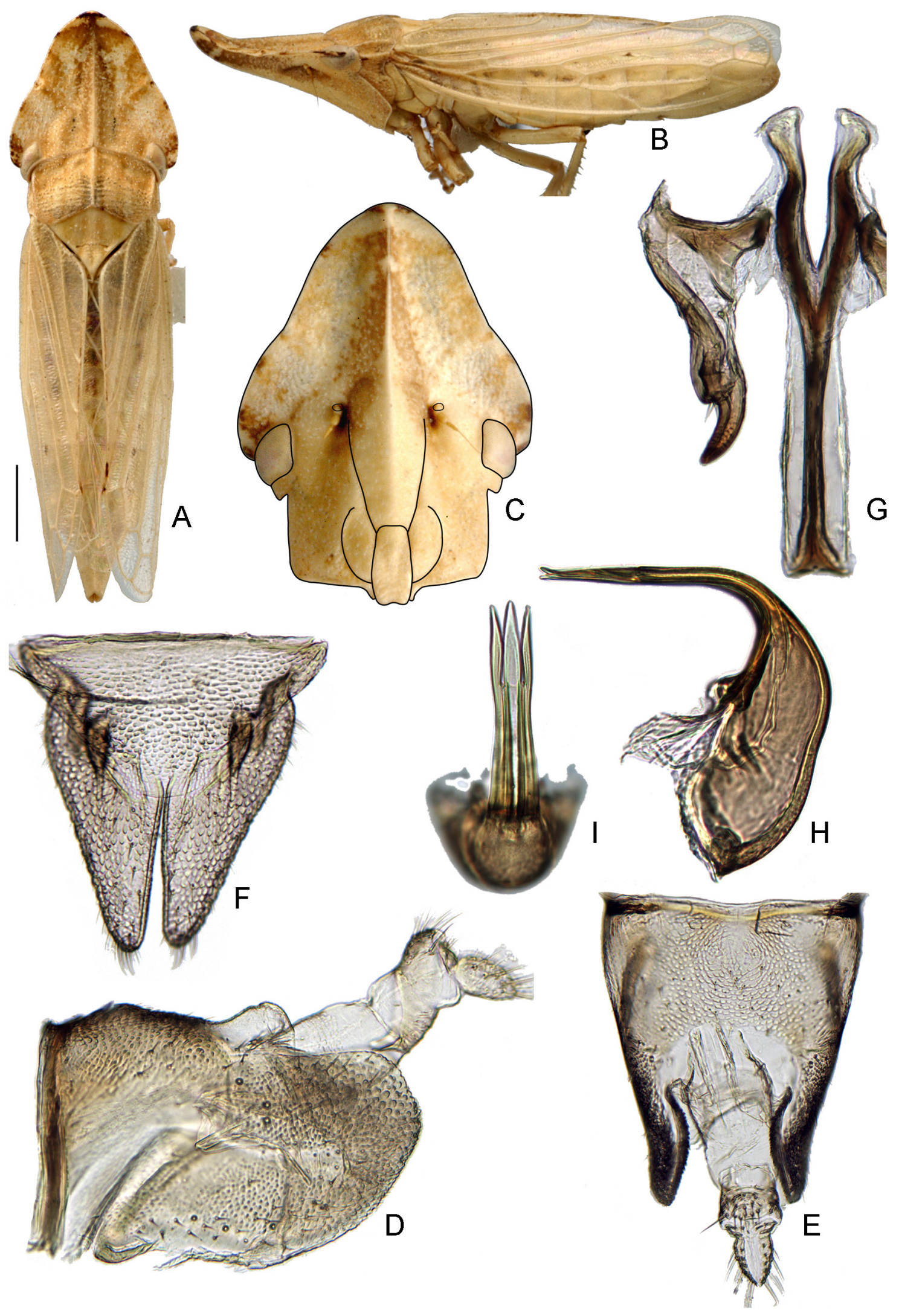

Fig. 22. Eupelicini (Eupelicina). Eupelix cuspidata (Fabricius, 1775). A-I. Standard views (see Material $\&$ Methods). 
hinge; with a single shaft and gonopore. Connective anterior arms somewhat divergent, Y-shaped; articulated with aedeagus.

Female Genitalia. Pygofer with macrosetae reduced or absent. Ovipositor not protruding far beyond pygofer apex. First valvula not strongly convex; dorsal sculpturing pattern reticulate or imbricate (with overlapping scales); sculpturing reaching dorsal margin or submarginal; without distinctly delimited ventroapical sculpturing. Second valvula broad, gradually tapered; with dorsal median tooth; teeth on apical $1 / 3$ or more; teeth small, regularly or irregularly shaped.

\title{
Geography and ecology
}

Distribution: Palearctic region.

\section{Remarks}

Eupelicina contains the type genus and its 2 included species.

\section{Included genera}

Eupelix Germar, 1821

\author{
Paradorydiina Evans, 1936
}

Fig. 23

Type genus: Paradorydium Kirkaldy, 1901.

= Dorydiini Fieber, 1872 (Type: Dorydium Burmeister, 1839; suppressed).

\section{Diagnosis}

Paradorydiina are medium sized to large, somewhat to very elongate, produced leafhoppers, usually whitish, greenish, or brownish in color. They can be distinguished from Eupelicina by the crown not extending over the eyes anteriorly, ocelli on the anterior margin of the head, lateral margin of the pronotum not carinate, frontoclypeus without a median anterior carina, forewing appendix reduced or absent, forewing marginal vein incomplete, hind wing marginal vein incomplete, male valve fused to the pygofer, style gracile, ovipositor extending far beyond the pygofer apex, first valvula dorsal sculpturing pattern maculose or granulose, second valvula without a dorsal median tooth and without dorsal teeth on apical $1 / 3$.

\section{Description}

Head. Head subequal to or wider than pronotum. Discal portion of crown punctate. Anterior margin of head foliaceous. Frontoclypeus not tumid; texture punctate. Clypellus parallel-sided or tapering apically; apex following or slightly surpassing normal curve of gena. Lorum subequal to or wider than clypellus near base. Antennal bases near upper or anterodorsal corners of eyes. Antennae short, less than $1.5 \mathrm{x}$ width of head. Gena strongly incised laterally (nearly forming right angle); without fine erect seta beside laterofrontal suture. Antennal ledges absent or weakly developed (carinate or weakly carinate). Ocelli present; close to eyes; on anterior margin of head.

Thorax. Pronotum lateral margin not carinate; lateral margin as long as or longer than basal width of eye.

WINGS. Forewing macropterous or submacropterous; acuminate; appendix absent or reduced; veins distinctly raised or carinate; without reflexed costal veins; A1-A2 crossvein absent; apical venation not 
ZAHNISER J.N. \& DIETRICH C.H., A review of the tribes of Deltocephalinae (Cicadellidae)

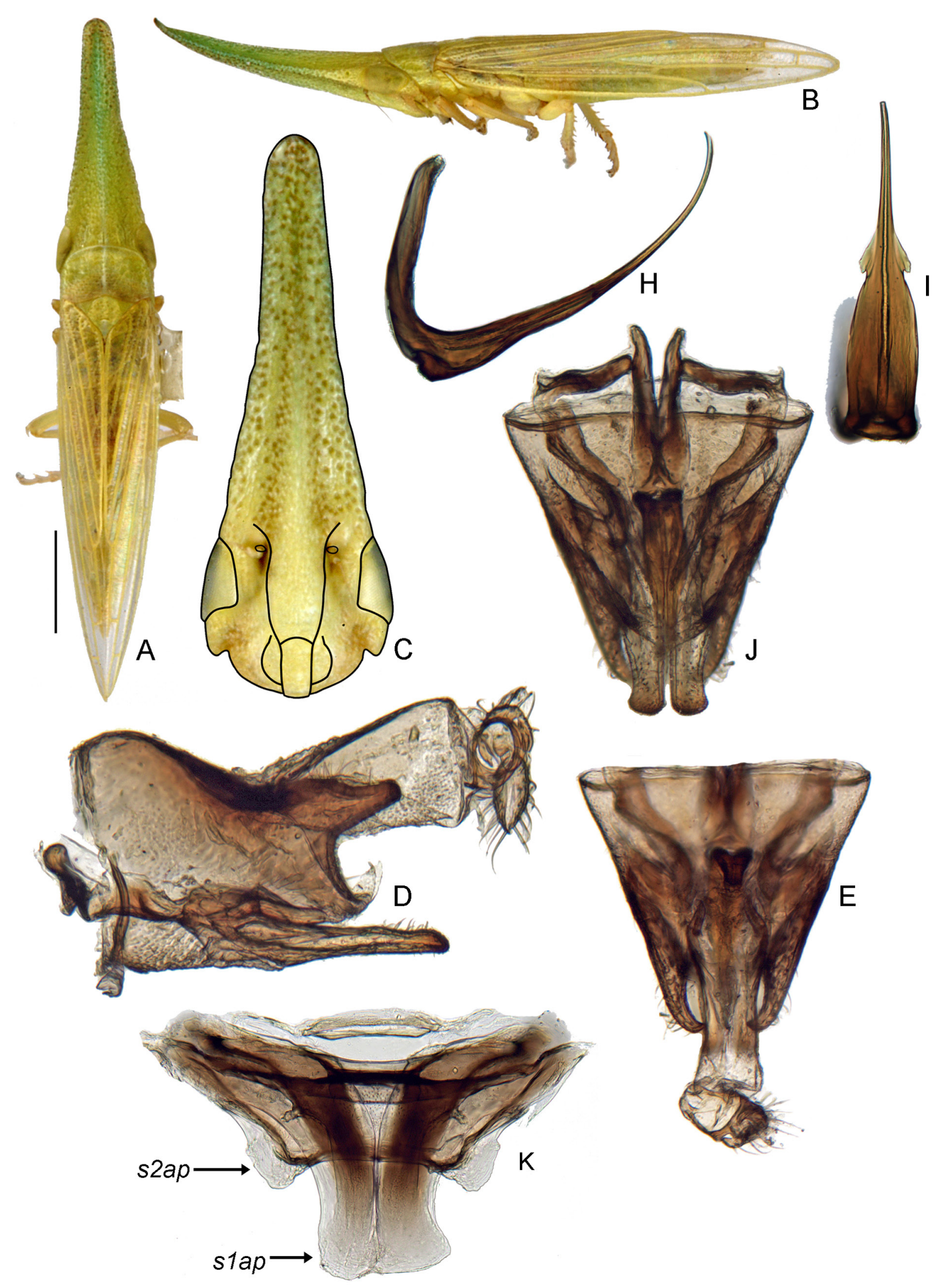

Fig. 23. Eupelicini (Parodorydiina). Paradorydium quadrigonum (Naudé, 1926). A-E, H-I. Standard views (see Material \& Methods). D. Pygofer shown with valve, plates, and internal genitalia. J. Ventral view of pygofer showing valve, plates, connective and styles. K. Ventral views of sternites I (slap) and II (s2ap). 
highly reticulate; marginal vein often absent or partially absent medial to $M 1+2$; hind wing marginal vein often absent.

LEGs. Profemur with AM1 absent or reduced; intercalary row reduced or absent; row AV with thin, hairlike setae, without setae, or with relatively long macrosetae. Protibia dorsal surface flat, AD and PD margins at $\sim 90^{\circ}$ angles but not carinate. Metafemur apex macrosetae 2+0. Metatarsomere I expanded apically, plantar setae simple, tapered.

MALE GENitALIA. Valve fused to pygofer. Pygofer basolateral membranous cleft absent, not membranous; macrosetae absent or reduced ( $\leq$ two rows). Subgenital plates free from each other and articulated with or fused to valve; without macrosetae. Style broadly bilobed basally, median anterior lobe pronounced; somewhat gracile, not fully sclerotized posteriad of median anterior lobe. Basal processes of the aedeagus/connective absent or reduced. Aedeagus without basal hinge; with a single shaft and gonopore. Connective anterior arms somewhat divergent, $\mathbf{Y}$ - or $\mathbf{U}$-shaped; articulated with aedeagus.

Female genitalia. Pygofer with macrosetae reduced or absent. Ovipositor protruding far beyond pygofer apex. First valvula not strongly convex; dorsal sculpturing pattern granulose or maculose; sculpturing submarginal; with ventroapical sculpturing distinctly delimited. Second valvula gradually broadened medially or subapically; without dorsal median tooth; teeth absent.

\section{Geography and ecology}

Distribution: Palearctic, Afrotropical, Oriental and Australian regions.

\section{Remarks}

Paradorydiina contains 6 genera and 62 species. Paradorydium and Chloropelix were included in the phylogenetic analyses here and were resolved together with very high branch support on a relatively long branch sister to Eupelix.

Sectoculus is transferred to Paradorydiina here. Morrison (1973a) established the genus and indicated that it belonged to Paradorydiini which he included in Hecalinae. It was listed in Hecalini by Oman et al. (1990) who apparently overlooked its placement by Morrison. It is similar morphologically to Paradorydium.

\section{Included genera}

Afralycisca Kocak, 1981

Chloropelix Lindberg, 1936

Mapochia Distant, 1910

Mapochiella Evans, 1966

Paradorydium Kirkaldy, 1901

Sectoculus Morrison, 1973 placement nov. (transferred from Hecalini)

Faltalini Zahniser \& Dietrich, 2010

Fig. 24

Type genus: Faltala Oman, 1938.

\section{Diagnosis}

Faltalini are small to large sized, somewhat to strongly dorsoventrally flattened, ivory, ochraceous, fuscous, yellowish, or brown leafhoppers; sometimes sexually dimorphic; dorsum sometimes with 5-6 more or less developed longitudinal ochraceous stripes bordered with fuscous or dark coloring; stripes may be 
present on head, pronotum, wings, and/or abdomen. They resemble Hecalini in the following characters: head often produced or parabolic in shape; first valvula dorsal sculpturing pattern submarginal; first valvula relatively straight; first valvula with distinctly delimited apicoventral sculptured area; second valvula without dorsal teeth. Faltalini can be distinguished from Hecalini and other Deltocephalinae by the following combination of characters: dorsoventrally flattened shape, head often produced, anterior margin of head often foliaceous or with carinae or striae, ocelli often distant from eyes (close to eyes in Hecalini), sometimes with distinctive color pattern, first valvula dorsal sculpturing pattern maculose to granulose, first valvula with distinctly delimited apicoventral sculpturing (usually not as long as in Hecalini and Dorycephalini), first valvula not humpbacked dorsally (humpbacked in Hecalini and Dorycephalini), second valvula straight to somewhat convex ventrally (concave in Hecalini and Dorycephalini), second valvula without dorsal teeth.

\section{Description}

HEAD. Head subequal to or wider than pronotum. Discal portion of crown glabrous with radial or longitudinal striae or shagreen. Anterior margin of head shagreen, glabrous, striate, or foliaceous. Frontoclypeus not tumid; texture shagreen or glabrous. Clypellus parallel-sided or tapering apically; apex following or slightly surpassing normal curve of gena. Lorum subequal to or wider than clypellus near base. Antennal bases near middle or posteroventral (lower) corners of eyes or near anterodorsal (upper) corners of eyes (some Tenucephalus, Bonamus). Antennae short, less than $1.5 \mathrm{x}$ width of head or long (Tenucephalus, Bonamus). Gena obtusely incised laterally; with fine erect seta beside laterofrontal suture. Antennal ledge absent. Ocelli absent or reduced or present; close to (Tenucephalus) or distant from eyes; on anterior margin of head.

Thorax. Pronotum lateral margin carinate; lateral margin shorter (Tenucephalus, Bonamus) or longer than basal width of eye.

Wings. Forewings of both sexes brachypterous, quadrate, coriaceous, venation indistinct to reticulate; or males submacropterous or macropterous (Acrolithus, Hecullus) and females brachypterous to submacropterous; or males and females macropterous (Tenucephalus, Bonamus); if macropterous, appendix restricted to anal margin; with 3 anteapical cells or with 2 anteapical cells; veins not raised; without reflexed costal veins; A1-A2 crossvein absent.

Legs. Protrochanter often with stout ventroapical seta. Profemur with AM1 seta only; intercalary row with one row of five or more fine setae; row $\mathrm{AV}$ with short, stout setae or with relatively long macrosetae. Protibia dorsal surface rounded, convex. Metafemur apex macrosetae $2+2$ or $2+2+1$. Metatarsomere I not expanded apically, plantar setae simple, tapered.

MALE Genitalia. Valve articulated with pygofer; lateral margin short, articulating with pygofer at a point. Pygofer basolateral membranous cleft present; macrosetae absent or reduced ( $\leq$ two rows) or macrosetae well differentiated into several rows. Subgenital plates free from each other; articulated with valve; macrosetae irregularly arranged, uniseriate laterally, or reduced or absent. Style broadly bilobed basally, median anterior lobe pronounced. Basal processes of the aedeagus/connective absent or reduced or present (Tenucephalus). Aedeagus with single shaft and gonopore. Connective anterior arms somewhat divergent, Y-shaped, or closely appressed anteriorly, linear shaped; articulated with or rarely fused (some Tenucephalus) to aedeagus.

Female genitalia. Pygofer with macrosetae reduced or absent. Ovipositor protruding (Tenucephalus, Bonamus) or not protruding far beyond pygofer apex. First valvula not strongly convex; dorsal sculpturing pattern granulose or maculose; sculpturing submarginal; with ventroapical sculpturing 


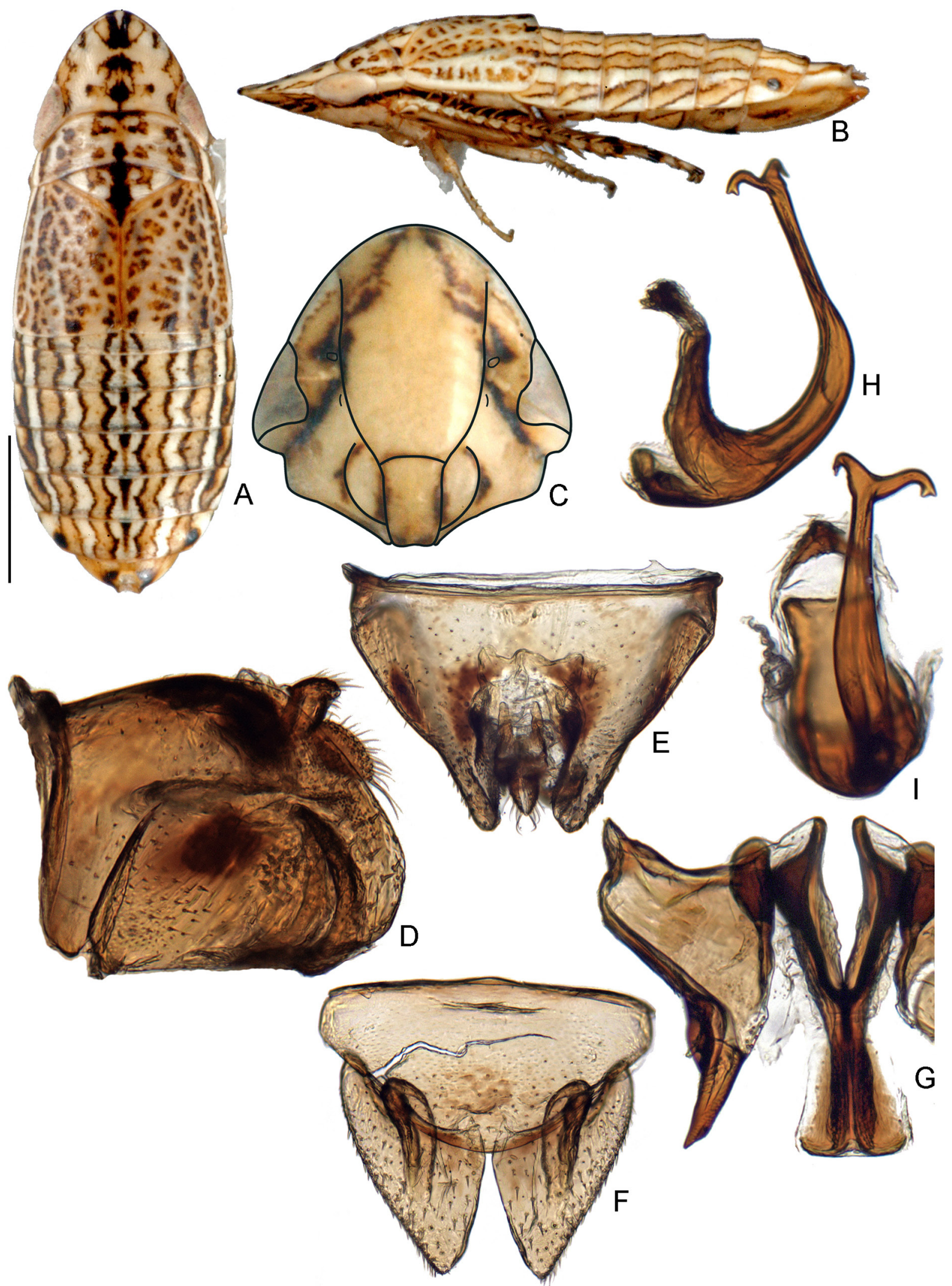

Fig. 24. Faltalini. Clorindaia cyphora Blocker \& Fang, 1992. A-I. Standard views (see Material \& Methods). 
distinctly delimited. Second valvula broad, gradually tapered or gradually broadened medially or subapically; without dorsal median tooth; teeth absent.

\section{Geography and ecology}

Distribution: Nearctic and Neotropical regions. Known hosts are grasses. Tenucephalus spp. have been collected at lights. Many species appear to be predominantly or completely brachypterous, some are sexually dimorphic for wing length (Acrolithus, Hecullus), and some are macropterous (Bonamus, Hecalocorica, Tenucephalus).

\section{Remarks}

Faltalini contains 10 genera and 26 species and is distributed in the New World from the southwestern United States to Argentina and Chile. Tenucephalus and Bonamus form a morphologically distinct group within the tribe, distinguished by the following characters: body long and slender; both sexes macropterous; ovipositor extending well beyond pygofer apex. Although including these genera makes the tribe as a whole more difficult to characterize morphologically, some characters, especially of the female genitalia, support a relationship to other Faltalini and 28S rDNA data very strongly support the monophyly of the tribe (Zahniser \& Dietrich 2010).

Egenus Oman, 1938 is removed from the tribe here. It was tentatively included by Zahniser \& Dietrich (2010) but subsequent observations of the female revealed that the genus does not possess the characters of the ovipositor that help to distinguish the tribe. It is transferred to Athysanini. A preliminary phylogenetic study of Hecalini (Catanach, unpublished) suggests that Egenus is related to Arrugada Oman, 1938.

\section{Selected references}

DeLong \& Thambimuttu (1973), Linnavuori \& DeLong (1977a), Blocker \& Fang (1992), Zahniser \& Webb (2004), Marino de Remes Lenicov \& Paradell (2009), Zahniser \& Dietrich (2010).

\section{Included genera}

Acrolithus Freytag \& Ma, 1988

Aequecephalus DeLong \& Thambimuttu, 1973

Bonamus Oman, 1938

Clorindaia Linnavuori, 1975

Faltala Oman, 1938

Hecalocorica Nielson, 1996

Hecullus Oman, 1949

Kramerana DeLong \& Thambimuttu, 1973

Tenucephalus DeLong, 1944

Virganana DeLong \& Thambimuttu, 1973

Fieberiellini Wagner, 1951

Fig. 25

Type genus: Fieberiella Signoret, 1880.

$=$ Synophropsini Ribaut, 1952.

\section{Diagnosis}

Fieberiellini are medium sized, tawny to brown leafhoppers. They can be identified by the forewing lacking an appendix, forewings not overlapping, connective T-shaped and with a distinct stem, style 
linear or not broadly bilobed basally, and female sternite VIII not concealed and folded beneath sternite VII and movably articulated with sternite VII*.

* Sternite VIII is almost always folded beneath and concealed by sternite VII in Deltocephalinae and is often membranous or reduced; thus, when the pygofer and ovipositor are in their resting positions, sternite VII appears to be the one immediately basad of the genital capsule. Meyer-Arndt \& Remane (1992b) pointed out the unique configuration in Fieberiellini but identified the entire structure (comprising sternites VII and VIII) as sternite VII.

\section{Description}

Head. Head subequal to or wider than pronotum. Discal portion of crown glabrous with radial or longitudinal striae. Anterior margin of head shagreen or with numerous transverse striations or carinae. Frontoclypeus not tumid; texture shagreen. Clypellus widening apically; apex following or slightly surpassing normal curve of gena. Lorum subequal to or wider than clypellus near base. Antennal bases near middle or posteroventral (lower) corners of eyes. Antennae short, less than $1.5 \mathrm{x}$ width of head. Gena obtusely incised laterally; with fine erect seta beside laterofrontal suture. Antennal ledge weakly developed (carinate or weakly carinate). Ocelli present, close to eyes, on anterior margin of head.

Thorax. Pronotum lateral margin carinate; lateral margin shorter than basal width of eye.

WINGS. Forewing macropterous; appendix absent or reduced; with 3 anteapical cells; veins not raised; without reflexed costal veins; A1-A2 crossvein present or absent.

Legs. Profemur with AM1 seta only or with one or more additional proximal setae; intercalary row with one row of five or more fine setae; row AV with relatively long macrosetae. Protibia dorsal surface rounded, convex. Metafemur apex macrosetae $2+2+1$. Metatarsomere I not expanded apically; plantar setae simple, tapered.

MaLe Genitalia. Valve articulated with pygofer; lateral margin short, articulating with pygofer at a point. Pygofer basolateral membranous cleft present; macrosetae well differentiated into several rows. Subgenital plates free from each other; articulated with valve; macrosetae irregularly arranged, uniseriate laterally, with two lateral rows of macrosetae, or with some irregularly arranged macrosetae near lateral margin. Style linear, median anterior lobe not pronounced. Basal processes of the aedeagus/ connective absent or reduced. Aedeagus with single shaft and gonopore. Connective anterior arms widely divergent, T-shaped, with darkly pigmented ventromedial ridge contrasting with more lightly pigmented dorsolateral areas; stem well-developed; articulated with aedeagus. Segment X sclerotized dorsally and laterally; sometimes large; sometimes with long ventrally directed processes.

Female genitalia. Pygofer with numerous macrosetae. Ovipositor not protruding far beyond pygofer apex. Sternite VIII not concealed and folded beneath sternite VII, movably articulated with sternite VII. First valvula convex; dorsal sculpturing pattern strigate; sculpturing reaching dorsal margin; without distinctly delimited ventroapical sculpturing. Second valvula abruptly broadened medially or subapically; without dorsal median tooth; teeth on apical 1/3 or more; teeth large, regularly shaped or small, regularly or irregularly shaped.

\section{Geography and ecology}

Distribution: endemic to the Palearctic region and adventive in the Nearctic region (Fieberiella florii Stål, 1864 in North America). Fieberiellini are most diverse in the southern Palearctic region, especially the Mediterranean subregion, and have host plants in a number of different families as documented by Meyer-Arndt \& Remane (1992b). 


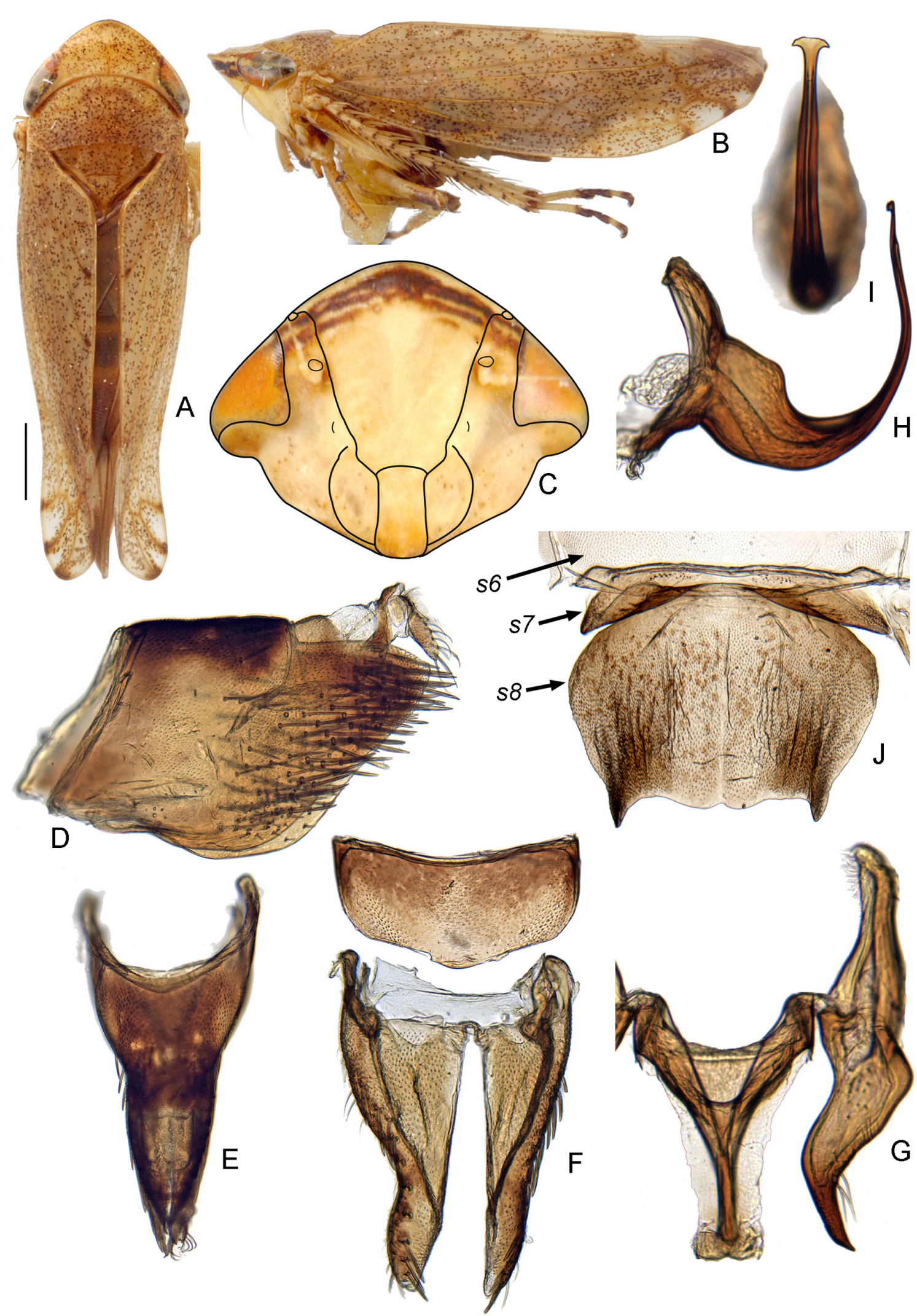

Fig. 25. Fieberiellini. A-C, J. Fieberiella florii (Stål, 1864). D-I. Placotettix taeniatifrons (Kirschbaum, 1868). A-I. Standard views (see Materials \& Methods). F. Plates shown slightly obliquely and valve shown separated from plates. J. + sternites VI-VIII $(s 6-s 8)$. 


\section{Remarks}

Fieberiellini contains 10 genera and 48 valid species. Meyer-Arndt \& Remane (1992a, b) produced a detailed revision of the tribe in which they illustrated the species, documented host plants and geographic ranges, clarified the morphological characterization and circumscription of the tribe, and proposed phylogenetic relationships among the genera. Phylogenetic analyses suggest that Fieberiellini is a relatively early diverging lineage of Deltocephalinae, and retains plesiomorphic characteristics of the male genitalia. It appears to be related to the monotypic Neotropical tribe Luheriini, with which it shares some morphological characteristics. However, the divergent 28S rDNA sequence of Luheria constricta and difficulty in amplifying some regions have confounded an exact and confident phylogenetic placement of the group. Fieberiella florii is adventive in North America and is a vector of the western strain of North American aster yellows, Western and Eastern X-disease of peach, and Western X-disease yellow leaf roll strain. Cechenotettix quadrinotatus (Mulsant \& Rey, 1855) is a vector of yellow decline of Lavandula.

Phycotettix was listed in Fieberiellini by Oman et al. (1990). It was not treated by Meyer-Arndt \& Remane (1992a, b ) and is removed from the tribe here and placed in Athysanini. It does not possess any of the characters that define the tribe.

\section{Selected references}

Meyer-Arndt \& Remane (1992a, b), Dmitriev (2003).

\section{Included genera}

Cechenotettix Ribaut, 1942

Docotettix Ribaut, 1948

Dohukia Meyer-Arndt \& Remane, 1992

Ericotettix Lindberg, 1960

Fieberiella Signoret, 1880

Habrostis Dubovsky, 1966

Heliotettix Rodrigues, 1968

Parafieberiella Dlabola, 1974

Placotettix Ribaut, 1942

Synophropsis Haupt, 1926

Goniagnathini Wagner, 1951

Fig. 26

Type genus: Goniagnathus Fieber, 1866.

\section{Diagnosis}

Goniagnathini are medium sized to large, squat, robust leafhoppers. They can be identified by the broad head, anterior margin of head glabrous, large forewing appendix (in macropterous individuals), subgenital plates fused to each other, valve apparently absent or fused to subgenital plates, style with broad basal part articulated with linear or modified apical part, and connective fused to the aedeagus.

\section{Description}

HEAD. Head subequal to or wider than pronotum; crown wide, parallel-margined. Discal portion of crown glabrous with radial or longitudinal striae. Anterior margin of head glabrous or irregularly textured. Frontoclypeus not tumid; texture glabrous, rugose, or shagreen. Clypellus widening apically; apex following or slightly surpassing normal curve of gena. Lorum subequal to or wider than clypellus 
near base. Antennal bases near middle or posteroventral (lower) corners of eyes. Antennae short, less than $1.5 \mathrm{x}$ width of head. Gena obtusely incised laterally; with fine erect seta beside laterofrontal suture. Antennal ledge weakly developed (carinate or weakly carinate). Ocelli present; close to eyes; on anterior margin of head.

THorax. Pronotum lateral margin carinate; lateral margin shorter than basal width of eye.

Wings. Forewing usually macropterous, sometimes brachypterous; if macropterous, appendix large, extending around wing apex; with 3 anteapical cells; veins not raised or raised; veins sometimes pustulate; without reflexed costal veins; A1-A2 crossvein present.

LEGS. Profemur with AM1 seta only; intercalary row with one row of five or more fine setae; row AV with relatively long macrosetae. Protibia dorsal surface rounded, convex. Metafemur apex macrosetae $2+2+1$. Metatarsomere I not expanded apically; plantar setae simple, tapered.

Male genitalia. Valve fused to subgenital plates or obscure. Pygofer basolateral membranous cleft present; macrosetae well differentiated into several rows. Subgenital plates fused to each other; with or without macrosetae. Style linear, median anterior lobe not pronounced or broadly bilobed basally, median anterior lobe pronounced; broad basal part articulated with linear or modified apical part, with cleft between the two. Basal processes of the aedeagus/connective absent or reduced. Aedeagus with single shaft and gonopore. Connective anterior arms somewhat divergent, $\mathbf{Y}$ - or $\mathbf{U}$-shaped, or closely appressed anteriorly, linear shaped; sometimes highly reduced in size; fused to aedeagus.

Female Genitalia. Pygofer with numerous macrosetae. Ovipositor not protruding far beyond pygofer apex. First valvula convex; dorsal sculpturing pattern strigate, concatenate, or reticulate; sculpturing reaching dorsal margin; without distinctly delimited ventroapical sculpturing. Second valvula slender throughout; without dorsal median tooth; teeth on apical $1 / 3$ or more or teeth restricted to apical $1 / 4$ or less; teeth small, regularly or irregularly shaped.

\section{Geography and ecology}

Distribution: Palearctic, Afrotropical, Oriental and Australian. Species are found in arid and grassland regions of the Old World where they feed on herbs and shrubs (e.g., Artemisia, Tamarix).

\section{Remarks}

Goniagnathini contains 4 genera and 60 species. Goniagnathini is an early-diverging lineage and appears to have diverged from the stem group of Deltocephalinae at nearly the same time as Acinopterini, Fieberiellini, and Luheriini. It has a unique configuration of the style, with a broad base articulated to a linear or modified apical part.

\section{Selected references}

Linnavuori (1978b), Dash \& Viraktamath (2001), Dmitriev (2003), Fletcher \& Zahniser (2008), Duan et al. (2009), Viraktamath \& Gnaneswaran (2009).

\section{Included genera}

Goniagnathus Fieber, 1866

Megalopsius Emeljanov, 1961

Raunothus Dlabola, 1987

Tamaricades Emeljanov, 1962 


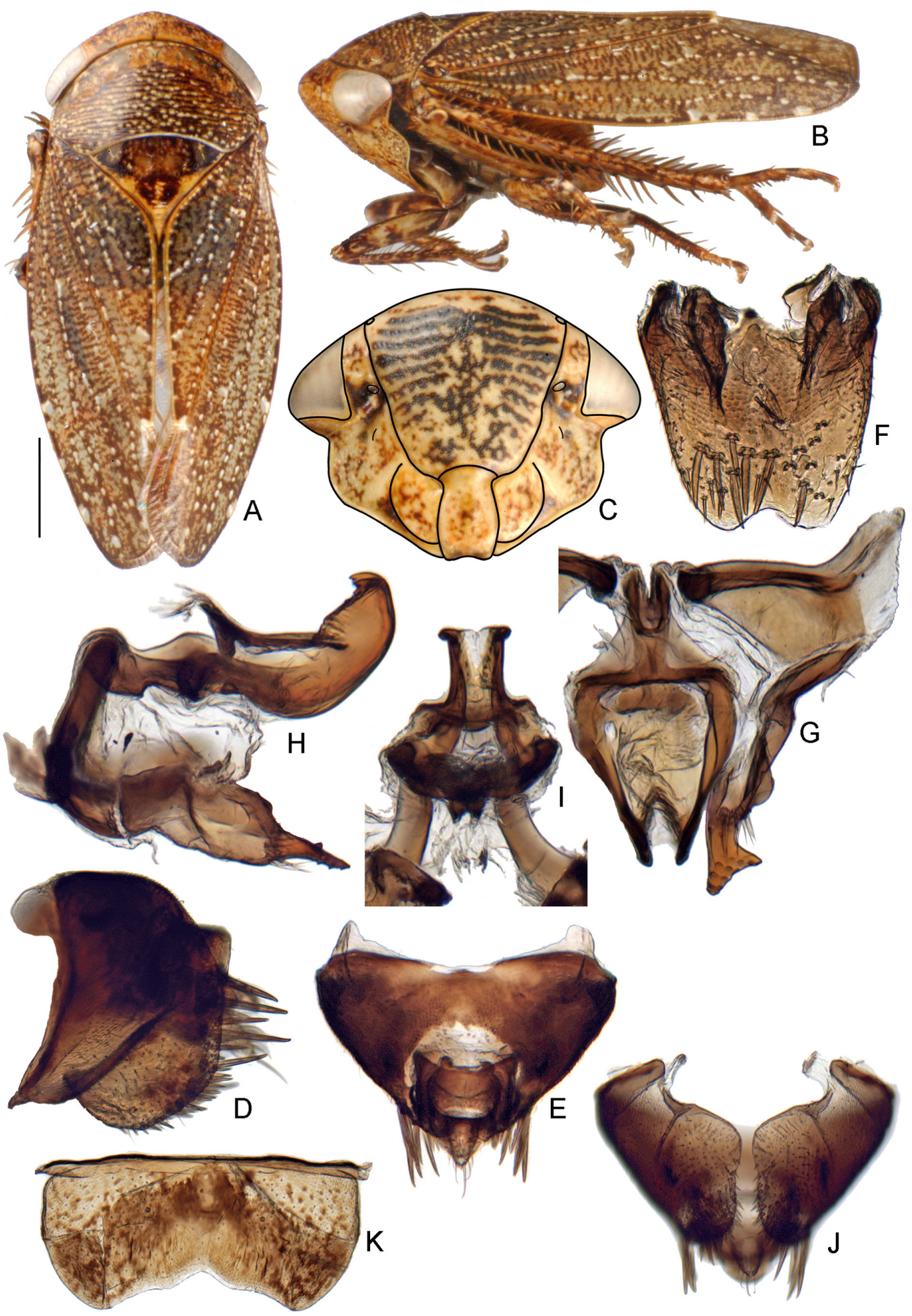

Fig. 26. Goniagnathini. A-B, D-K. Goniagnathus emeljanovi sp. nov. C. G. rugulosus (Haupt, 1917). A-I. Standard views (see Material \& Methods). G. Connective and style shown with aedeagus fused to connective. H. Aedeagus shown fused to connective, style also shown. I. Caudal view of aedeagus/ connective shown with median arms of styles extending ventrally. J. Ventral view of pygofer. K. ㅇ sternite VII. 
Hecalini Distant, 1908

Figs 27,28

Type genus: Hecalus Stål, 1864.

$=$ Reuteriellini Evans, 1947.

\section{Diagnosis}

Hecalini are medium sized to large, somewhat to strongly dorsoventrally flattened, stramineous, yellow, green, or brown leafhoppers, sometimes with bright orange or reddish markings. They can be identified by the produced and parabolically shaped head, dorsoventrally flattened body, lateral margin of pronotum as long as or longer than the basal width of the eye, ocelli closer to they eyes than the laterofrontal sutures, apodemes of male sternite I long and relatively narrow, apodemes of male sternite II broad and well-developed, male pygofer often produced or pointed posterodorsally, segment $\mathrm{X}$ withdrawn into the pygofer, ventral margins of the male pygofer often lobate, aedeagus often with one or two pairs of apical processes, first valvula dorsal sculpturing granulose to maculose and submarginal, first valvula often with distinctly delimited ventroapical sculpturing, second valvula usually without teeth, humpbacked dorsally, and concave ventrally.

\section{Description}

HEAD. Head subequal to or wider than pronotum; often produced anteriorly and/or parabolically shaped. Discal portion of crown glabrous with radial or longitudinal striae, shagreen or granulose. Anterior margin of head shagreen, glabrous, irregularly textured, foliaceous, with a single transverse carina, or with 2 or 3 parallel carinae. Frontoclypeus tumid or flat; texture shagreen. Clypellus parallel-sided, tapering apically, or rarely widening apically; apex following or slightly surpassing normal curve of gena. Lorum narrower than, subequal to, wider than clypellus near base. Antennal bases near middle or posteroventral (lower) corners of eyes. Antennae short, less than $1.5 \mathrm{x}$ width of head. Gena obtusely incised laterally or strongly incised laterally (nearly forming right angle); with or without (Attenuipyga, some Glossocratus) fine erect seta beside laterofrontal suture. Antennal ledge absent. Ocelli present; close to eyes, closer to eyes than laterofrontal sutures; on anterior margin of head.

THORAX. Pronotum lateral margin carinate; lateral margin as long as or longer than basal width of eye.

WINGS. Forewing brachypterous to macropterous; if macropterous, appendix restricted to anal margin or rarely large, extending around wing apex; with 3 anteapical cells; veins not elevated or rarely elevated (Attenuipyga); without reflexed costal veins; A1-A2 crossvein absent or present; A1 and A2 sometimes confluent (Attenuipyga [Dorycara]).

LEGS. Profemur with AM1 seta only or rarely with AM1 and with one or more additional proximal setae (some Glossocratus) or AM1 absent (Attenuipyga); intercalary row with one row of five or more fine setae or setae scattered, not in one row, or setae absent or reduced; row AV with short, stout setae or with relatively long macrosetae. Protibia dorsal surface rounded, convex. Metafemur apex macrosetae $2+2+1,2+2+1$ and with additional proximal macrosetae (some Glossocratus), or 2+0 (Attenuipyga). Metatarsomere I not expanded apically or expanded apically; plantar setae simple, tapered.

MaLe genitalia. Apodemes of sternite I usually long, usually as long as those of sternite II, relatively narrow. Apodemes of sternite II large, broad (exception: Glossocratus). Valve articulated with pygofer; lateral margin short, articulating with pygofer at a point. Pygofer basolateral membranous cleft present; macrosetae well differentiated into several rows; often produced or pointed posterodorsally, with 'anal tube' withdrawn into pygofer; ventral margins often lobate. Subgenital plates free from each other; 
articulated with valve; macrosetae scattered, irregularly arranged or uniseriate laterally. Style broadly bilobed basally, median anterior lobe pronounced. Basal processes of the aedeagus/connective absent or reduced. Aedeagus with single shaft and gonopore; often with one or two pairs of apical processes, or rarely with preapical processes. Connective anterior arms somewhat divergent, Y- or U-shaped; articulated with aedeagus.

Female genitalia. Pygofer with macrosetae absent, reduced, or with numerous macrosetae. Ovipositor usually protruding or sometimes not protruding far beyond pygofer apex. First valvula not strongly convex; dorsal sculpturing pattern granulose or maculose; sculpturing submarginal or rarely reaching dorsal margin; usually with ventroapical sculpturing distinctly delimited. Second valvula gradually broadened medially or subapically; humpbacked dorsally; concave ventrally; usually without dorsal median tooth (except Glossocratus); dorsal teeth usually absent but if present (some Glossocratus), teeth small, regularly or irregularly shaped, on apical $1 / 3$ or more.

\section{Geography and ecology}

Distribution: cosmopolitan. Hecalini are grass feeders and are sometimes very brightly colored with yellow, green (in live specimens), or orange. Some are extremely elongate and dorsoventrally flattened, apparently an adaptation enhancing crypsis on their grass hosts.

\section{Remarks}

Hecalini contains 23 genera and 180 species. The three included exemplars (Hecalus, Attenuipyga and Glossocratus) were resolved as a monophyletic group here in MP analyses, but not in ML or Bayesian analyses. In the MP analysis, the partitioned Bremer support from the Histone H3 data was -14.4 which apparently strongly conflicted with the signal from the $28 \mathrm{~S}$ and morphology data sets $(\mathrm{DI}=7.7$ and 13.2, respectively). This conflict in signal likely influenced the results of the ML and Bayesian analyses which resolved different topologies for the hecalines included here. MP analyses of the combined data suggest that Hecalini is closely related to Arrugadini, but more data are needed to confirm this.

Hamilton (2000) revised the concept of Hecalini and excluded several genera that have only a superficial similarity to the tribe. He also pointed out several characters that are shared between Hecalini and "Dorycephalini", although of the latter he was apparently referring only to the New World Attenuipyga and Neoslossonia, and not to Dorycephalus which does not share several of the characters he mentioned. The characters listed by Hamilton (2000) and others noted here that are shared between Attenuipyga, Neoslossonia, and other Hecalini and not with Dorycephalus are listed in the Diagnosis. Based on their shared morphological characters and support from the molecular data, Attenuipyga and Neoslossonia are transferred to Hecalini here. Dorycephalini is maintained as a separate monotypic tribe.

Morrison (1973) revised the Oriental fauna and suggested that Clavena may belong to Ledrini based on the position of the ocelli stated in the original description of C. sulcata Melichar. He did not examine the type, so its inclusion in Hecalini has not been confirmed. Sectoculus is removed from Hecalini and placed in Eupelicini: Paradorydiina (see discussion under Paradorydiina).

\section{Selected references}

Linnavuori (1957, 1959, 1975), Morrison (1973), Linnavuori \& DeLong (1978c), Hamilton (2000), Dmitriev (2002).

Included subtribes: 
Glossocratina Dmitriev, 2002

Fig. 27

Type genus: Glossocratus Fieber, 1866.

\section{Diagnosis}

Glossocratina are medium sized to large brownish leafhoppers. They are distinguished from Hecalina by the laterofrontal sutures keeled above the antennal pits, gena sometimes without a fine erect seta beside the laterofrontal suture, apodemes of male sternite II not as developed as in Hecalina, ovipositor not protruding far beyond the pygofer apex, and second valvula with teeth. Nymphs can be distinguished from Hecalina by the oblique lateral carina extending from antennal pit to margin of head, outer margin of the gena angulate, anal tube with a long apical process, and body covered with short setae (Dmitriev, 2002).

\section{Description}

HEAD. Head subequal to or wider than pronotum. Discal portion of crown radially or longitudinally striate or shagreen. Anterior margin of head foliaceous or with 2 or 3 parallel carinae. Frontoclypeus tumid or not; texture shagreen. Laterofrontal sutures keeled above antennal pits. Clypellus parallelsided, tapering apically, or widening apically; apex following or slightly surpassing normal curve of gena. Lorum subequal to or wider than clypellus near base. Antennal bases near middle or posteroventral (lower) corners of eyes. Antennae short, less than $1.5 \mathrm{x}$ width of head. Gena strongly incised laterally (nearly forming right angle); with or without fine erect seta beside laterofrontal suture. Antennal ledges absent. Ocelli present; close to eyes; on anterior margin of head.

THORAX. Pronotum lateral margin carinate; lateral margin as long as or longer than basal width of eye.

WINGS. Forewing brachypterous to macropterous; appendix restricted to anal margin or large, extending around wing apex; with 3 anteapical cells; veins not raised; without reflexed costal veins; A1-A2 crossvein absent or present; apical venation not highly reticulate.

Legs. Profemur with AM1 seta only or with AM1 and with one or more additional proximal setae; intercalary row setae not in one row or scattered; row AV with relatively long macrosetae. Protibia dorsal surface rounded, convex. Metafemur apex macrosetae $2+2+1$ or $2+2+1$ and with additional proximal macrosetae. Metatarsomere I expanded apically; plantar setae simple, tapered.

Male Genitalia. Apodemes of male sternite II not as long as those of sternite I (sternite II apodemes less developed than in Hecalina). Valve articulated with pygofer; with short point of articulation with pygofer. Pygofer dorsoapical margin incised to near mid-length; basolateral membranous cleft present; macrosetae well differentiated into several rows. Subgenital plates free from each other; articulated with valve; macrosetae uniseriate laterally. Style broadly bilobed basally, median anterior lobe pronounced. Basal processes of the aedeagus/connective absent. Aedeagus without basal hinge; with a single shaft and gonopore. Connective anterior arms somewhat divergent, $\mathbf{Y}$ - or $\mathbf{U}$-shaped; articulated with aedeagus.

Female genitalia. Pygofer with numerous macrosetae. Ovipositor not protruding far beyond pygofer apex. First valvula not strongly convex; dorsal sculpturing pattern granulose or maculose; sculpturing reaching dorsal margin; without distinctly delimited ventroapical sculpturing. Second valvula very broad; gradually or abruptly broadened medially or subapically; with or without dorsal median tooth; teeth on apical $1 / 3$ or more; teeth small, regularly or irregularly shaped. 


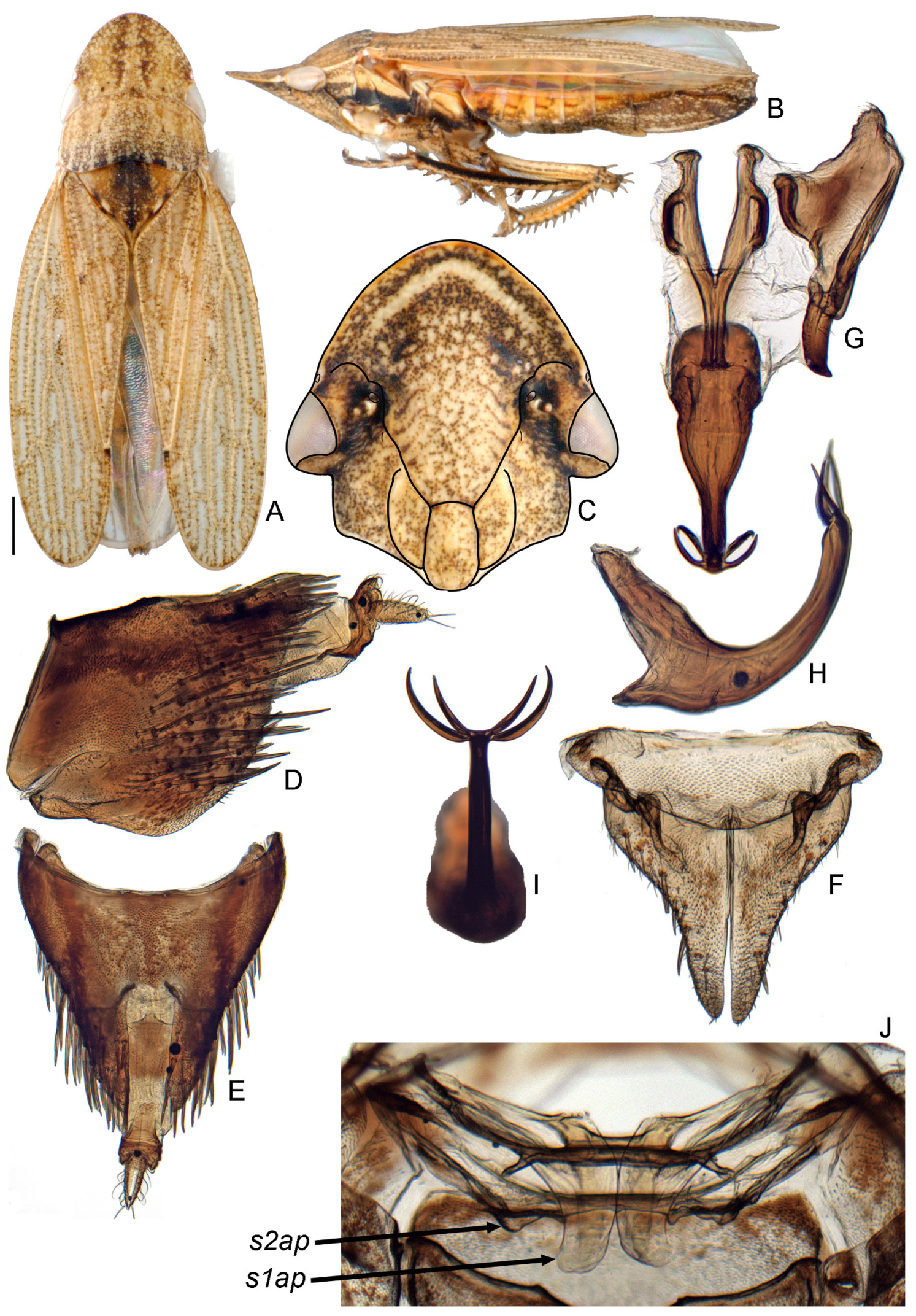

Fig. 27. Hecalini (Glossocratina). Glossocratus afzelii (Stål, 1854). A-I. Standard views (see Material $\&$ Methods). G. Connective and style shown with aedeagus. J. Dorsal view of $\lambda \hat{~ a b d o m i n a l ~ s t e r n i t e s ~}$ I-III (partial view of sternite III). slap = apodeme of sternite I, s2ap = apodeme of sternite II. 


\section{Geography and ecology}

Distribution: Palearctic, Afrotropical and Oriental regions. Glossocratina are grass feeders.

\section{Remarks}

Glossocratina contains the type genus and 29 species.

\section{Included genera}

Glossocratus Fieber, 1866

Hecalina Distant, 1908

Fig. 28

Type genus: Hecalus Stål, 1864.

\section{Diagnosis}

Hecalina are medium sized to large yellow, greenish, or brownish leafhoppers, sometimes with orange or reddish stripes. They are distinguished from Glossocratina by the unkeeled laterofrontal suture, gena with fine erect seta beside the laterofrontal suture, ovipositor extending far beyond the pygofer apex, and second valvula without dorsal teeth.

\section{Description}

HeAD. Head subequal to or wider than pronotum. Discal portion of crown radially or longitudinally striate or shagreen. Anterior margin of head shagreen, glabrous, irregularly textured, foliaceous, with a single transverse carina, or with 2 or 3 parallel carinae. Frontoclypeus tumid or not; texture shagreen. Clypellus parallel-sided or tapering apically; apex following or slightly surpassing normal curve of gena. Lorum distinctly narrower than clypellus near base. Antennal bases near middle or posteroventral (lower) corners of eyes. Antennae short, less than $1.5 \mathrm{x}$ width of head. Gena obtusely incised laterally or strongly incised laterally (nearly forming right angle); with fine erect seta beside laterofrontal suture. Antennal ledges absent. Ocelli present; close to eyes; on anterior margin of head.

Thorax. Pronotum lateral margin carinate; lateral margin as long as or longer than basal width of eye.

WINGS. Forewing brachypterous to macropterous; appendix restricted to anal margin; with 3 anteapical cells; veins not raised; without reflexed costal veins; A1-A2 crossvein absent or present; apical venation not highly reticulate.

Legs. Profemur with AM1 seta only; intercalary row with one row of five or more fine setae; row AV with short, stout setae. Protibia dorsal surface rounded, convex. Metafemur apex macrosetae $2+2+1$. Metatarsomere I not expanded apically or expanded apically, plantar setae simple, tapered.

Male genitalia. Apodemes of sternite I long, as long as those of sternite II, relatively narrow. Apodemes of sternite II large, broad. Valve articulated with pygofer; with short point of articulation with pygofer. Pygofer basolateral membranous cleft present; macrosetae well differentiated into several rows; anteroventral margins lobate; produced posterodorsally. Subgenital plates free from each other; articulated with valve; macrosetae scattered, irregularly arranged, or uniseriate laterally. Style broadly bilobed basally, median anterior lobe pronounced. Basal processes of the aedeagus/connective absent. Aedeagus without basal hinge; with a single shaft and gonopore; often with one or two pairs of apical or subapical processes. Connective anterior arms somewhat divergent, $\mathbf{Y}$ - or $\mathbf{U}$-shaped; stem often short; articulated with aedeagus. Segment $\mathrm{X}$ partially retracted into pygofer. 


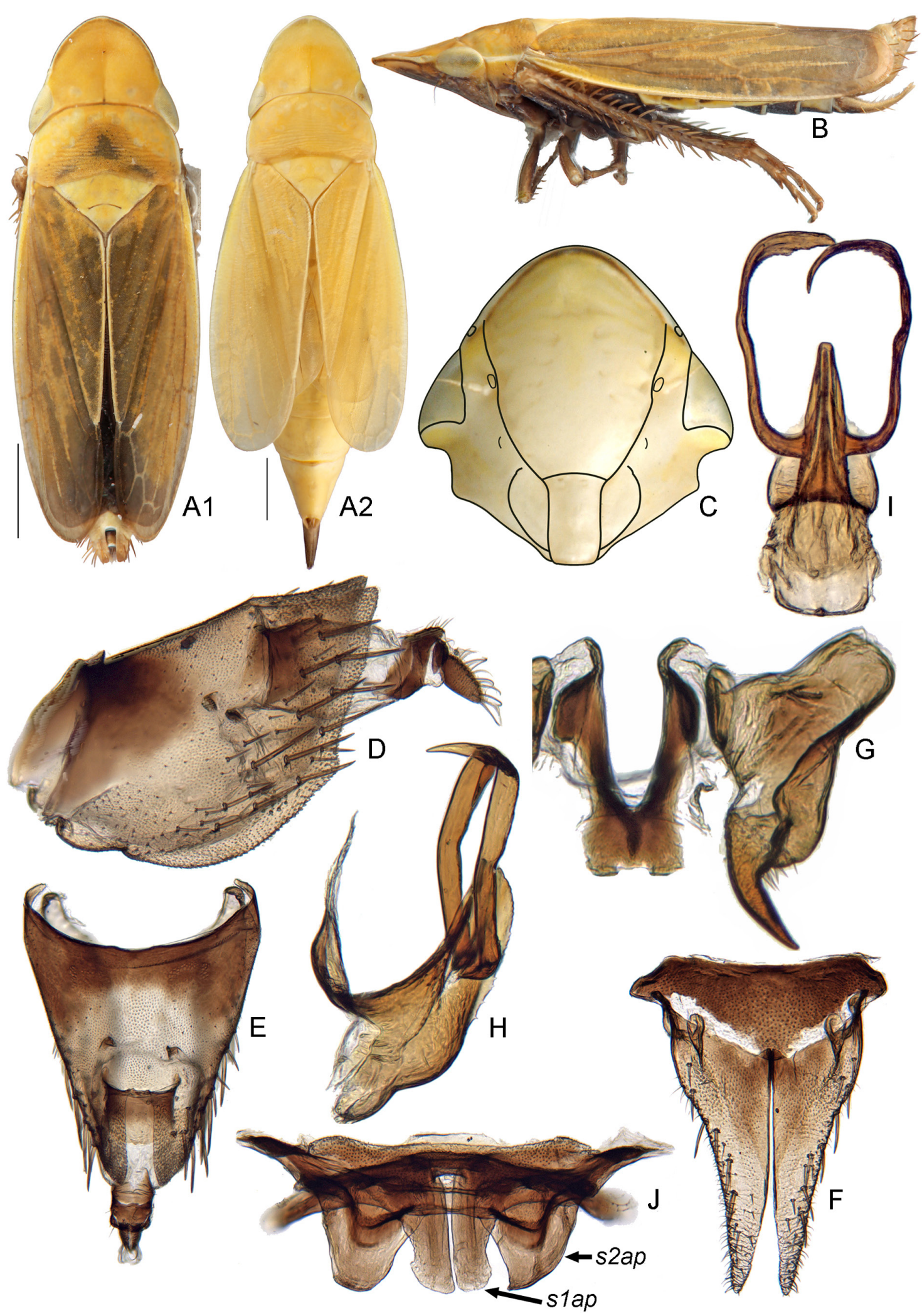

Fig. 28. Hecalini (Hecalina). A-B, D-I. Hecalus hamiltoni sp. nov. C. Hecalus viridis (Uhler, 1877). J. Attenuipyga platyrhynchus (Osborn, 1894). A1, §ิ. A2, ․ . A-I. Standard views (see Material \& Methods). J. Ventral views of sternites I and II. slap = apodeme of sternite I, $s 2 a p=$ apodeme of sternite II. 
Female genitalia. Pygofer with macrosetae reduced or absent. Ovipositor protruding far beyond pygofer apex. First valvula not strongly convex; humpbacked in shape; dorsal sculpturing pattern granulose or maculose; sculpturing submarginal; with ventroapical sculpturing distinctly delimited. Second valvula gradually broadened medially or subapically; humpbacked in shape; concave ventrally; without dorsal median tooth; teeth absent.

\section{Geography and ecology}

Distribution: cosmopolitan. Hecalina are grass feeders.

\section{Remarks}

Hecalina contains 22 genera and 151 species.

Except for Hecalus, Attenuipyga, and Neoslossonia, all genera were previously unplaced to subtribe and are included here in the subtribe Hecalina. Hecalus was previously the only included genus in the subtribe (Dmitriev 2002). Attenuipyga and Neoslossonia are transferred to Hecalina from Dorycephalini.

\section{Included genera}

Alospangbergia Evans, 1973 placement nov. (previously unplaced in Hecalini) Annidion Kirkaldy, 1905 placement nov. (previously unplaced in Hecalini) Attenuipyga Oman, 1949 placement nov. (transferred from Dorycephalini) Bordesia de Bergevin, 1929 placement nov. (previously unplaced in Hecalini) Cephalius Fieber, 1875 placement nov. (previously unplaced in Hecalini) Clavena Melichar, 1902 placement nov. (previously unplaced in Hecalini) Dicyphonia Ball, 1900 placement nov. (previously unplaced in Hecalini) Epicephalius Matsumura, 1908 placement nov. (previously unplaced in Hecalini) Hecalocratus Evans, 1966 placement nov. (previously unplaced in Hecalini) Hecalus Stål, 1864

Hecalusina He, Zhang \& Webb, 2008 placement nov. (previously unplaced in Hecalini) Jiutepeca Linnavuori \& DeLong, 1978 placement nov. (previously unplaced in Hecalini) Linnavuoriella Evans, 1966 placement nov. (previously unplaced in Hecalini) Lualabanus Linnavuori, 1975 placement nov. (previously unplaced in Hecalini) Memnonia Ball, 1900 placement nov. (previously unplaced in Hecalini) Neohecalus Linnavuori, 1975 placement nov. (previously unplaced in Hecalini) Neoslossonia Van Duzee, 1909 placement nov. (transferred from Dorycephalini) Parabolocratalis Evans, 1955 placement nov. (previously unplaced in Hecalini) Psegmatus Fieber, 1875 placement nov. (previously unplaced in Hecalini) Reuteriella Signoret, 1879 placement nov. (previously unplaced in Hecalini) Spangbergiella Signoret, 1879 placement nov. (previously unplaced in Hecalini) Thomsoniella Signoret, 1880 placement nov. (previously unplaced in Hecalini)

Hypacostemmini Linnavuori \& Al-Ne'amy, 1983

Fig. 29

Type genus: Hypacostemma Linnavuori, 1961.

Large, greenish leafhoppers. 


\section{Diagnosis}

Hypacostemmini are large, greenish leafhoppers. They can be identified by their large size $(7-10 \mathrm{~mm})$, highly reticulated apical venation of the forewing, appendix reduced in size, tectiform configuration of the forewings, and style apex foot-like, sharply angled medially with apex projecting laterally and darkly sclerotized.

\section{Description}

Head. Head subequal to or wider than pronotum. Discal portion of crown glabrous with radial or longitudinal striae. Anterior margin of head shagreen. Frontoclypeus not tumid; texture shagreen. Clypellus parallel-sided or widening apically; apex following or slightly surpassing normal curve of gena. Lorum subequal to or wider than clypellus near base. Antennal bases near middle or posteroventral (lower) corners of eyes. Antennae short, less than $1.5 \mathrm{x}$ width of head or long, $1.5 \mathrm{x}$ width of head or longer. Gena obtusely incised laterally; with fine erect seta beside laterofrontal suture. Antennal ledge weakly developed (carinate or weakly carinate). Ocelli present; close to eyes; on anterior margin of head.

THorax. Pronotum lateral margin carinate; lateral margin shorter than basal width of eye.

WINGS. Forewing macropterous; appendix restricted to anal margin, reduced in size; with 3 anteapical cells; veins not raised; without reflexed costal veins; A1-A2 crossvein absent or present; apical venation highly reticulate.

Legs. Profemur with AM1 seta only; intercalary row with one row of five or more fine setae; row AV with short, stout setae. Protibia dorsal surface rounded, convex. Metafemur apex macrosetae $2+2+1$. Metatarsomere I not expanded apically; plantar setae simple, tapered.

Male Genitalia. Valve articulated with pygofer; lateral margin short, articulating with pygofer at a point. Pygofer basolateral membranous cleft present; macrosetae well differentiated into several rows. Subgenital plates free from each other; articulated with valve; macrosetae uniseriate laterally. Style broadly bilobed basally; median anterior lobe pronounced; apex foot-like, sharply angled medially with apex projecting laterally, darkly sclerotized. Basal processes of the aedeagus/connective absent or reduced. Aedeagus with single shaft and gonopore. Connective anterior arms somewhat divergent, Y-or U-shaped; articulated with aedeagus.

Female genitalia. Pygofer with numerous macrosetae. Ovipositor not protruding far beyond pygofer apex. First valvula convex, dorsal sculpturing pattern strigate, concatenate, or reticulate; sculpturing reaching dorsal margin; without distinctly delimited ventroapical sculpturing. Second valvula abruptly broadened medially or subapically; with dorsal median tooth; teeth on apical $1 / 3$ or more; teeth small, regularly or irregularly shaped.

\section{Geography and ecology}

Distribution: Afrotropical (South Africa). Specimens have been collected at lights and swept from trees and bushes. Recorded host plant families include Urticaceae, Sapindaceae, and Anacardiaceae (Theron, 1987).

\section{Remarks}

Hypacostemmini contains the type genus and 5 species. Phylogenetic analyses suggest that Hypacostemma is related to a group of South African genera including Bonaspeiini and some undescribed genera sampled in the analyses here (Cameroon142 and Ghana114). This group is in turn related to Selenocephalini. 


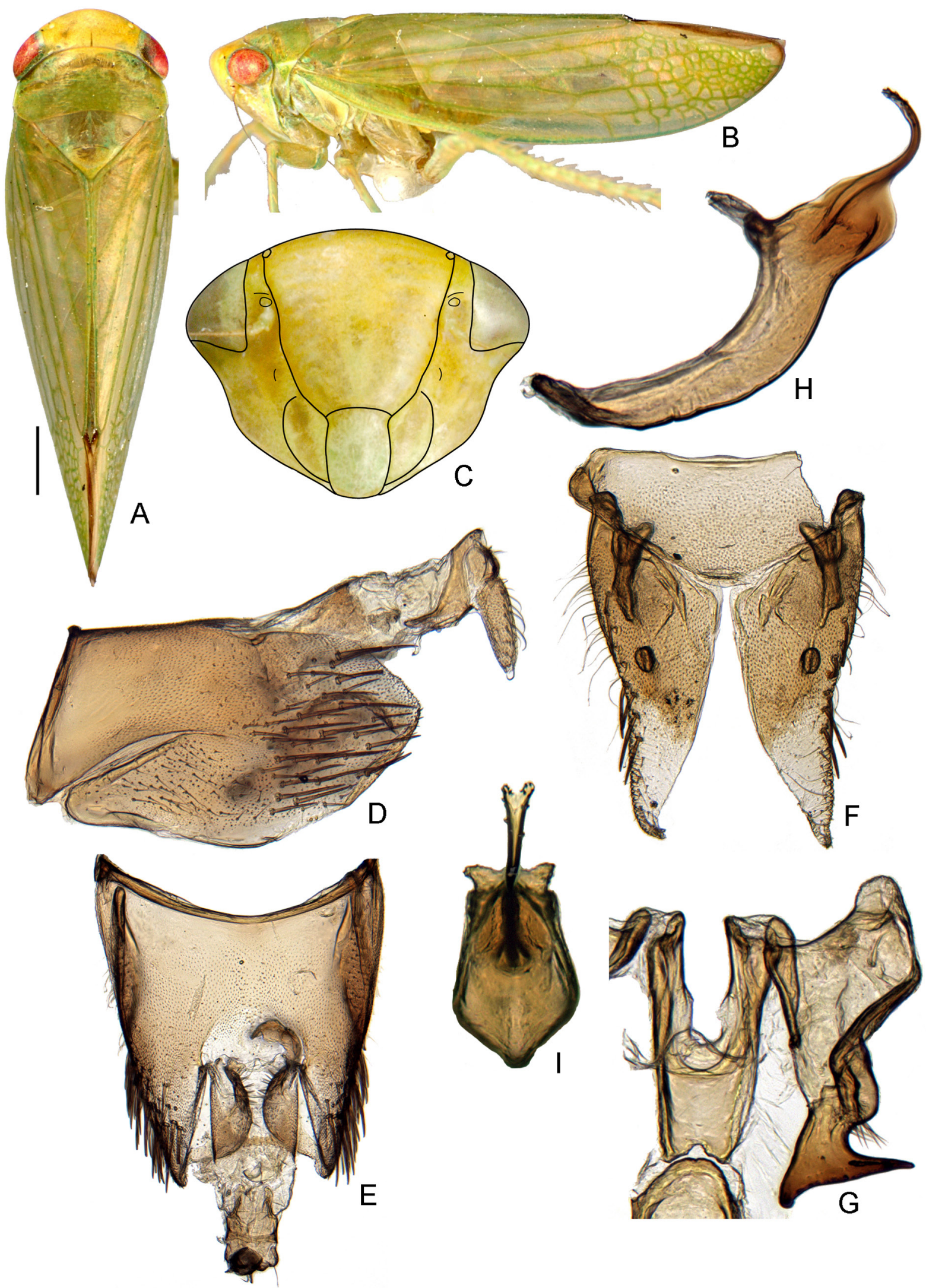

Fig. 29. Hypacostemmini. Hypacostemma viridissima Linnavuori, 1961. A-I. Standard views (see Material \& Methods). 
More detailed phylogenetic analyses of these genera, including more representatives of other genera in the diverse Bonaspeiini, are needed.

\section{Selected references}

Linnavuori \& Al-Ne'amy (1983), Theron (1987).

\section{Included genera}

Hypacostemma Linnavuori, 1961

Koebeliini Baker, 1897

Figs 30, 31

Type genus: Koebelia Baker, 1897.

$=$ Grypotini Haupt, 1929.

\section{Diagnosis}

Koebeliini are small to medium sized, yellow, light green, or brown leafhoppers. They can be identified by the ocelli distant from the eyes, clypellus long, narrow and extending well beyond the normal curve of the gena, and metatarsomere I with platellae on the plantar surface.

\section{Description}

Head. Head subequal to or wider than pronotum. Discal portion of crown glabrous with radial or longitudinal striae or shagreen. Anterior margin of head foliaceous (Koebeliina) or shagreen, with transverse striations, or carinate (Grypotina). Frontoclypeus not tumid; texture shagreen. Clypellus narrow; parallel-sided or tapering apically; apex far-surpassing normal curve of gena. Lorum subequal to or wider than clypellus near base. Antennal bases near middle or posteroventral (lower) corners of eyes or near anterodorsal (upper) corners of eyes. Antennae short, less than $1.5 \mathrm{x}$ width of head. Gena obtusely incised laterally, with fine erect seta beside laterofrontal suture. Antennal ledges absent. Ocelli present; distant from eyes; on anterior margin of head (Grypotina) or on face (Koebeliina).

THORAX. Pronotum lateral margin carinate or not; lateral margin shorter than basal width of eye.

WINGS. Forewing macropterous or submacropterous; appendix absent, reduced, or restricted to anal margin; with 3 anteapical cells; veins not raised or slightly raised; without reflexed costal veins; A1-A2 crossvein absent.

Legs. Profemur with AM1 seta only; intercalary row with one row of five or more fine setae or reduced or absent; row AV with short, stout setae or with relatively long macrosetae. Protibia dorsal surface rounded, convex or flat, $\mathrm{AD}$ and $\mathrm{PD}$ margins at $\sim 90^{\circ}$ angles but not carinate. Metafemur apex macrosetae $2+2+1,2+2,2+1,2+1+1$, or $2+2+1+1$. Metatarsomere I not expanded apically; plantar surface with one or more platellae.

Male Genitalia. Valve articulated with pygofer; lateral margin short, articulating with pygofer at a point. Pygofer basolateral membranous cleft present; macrosetae absent or reduced ( $\leq$ two rows) or well differentiated into several rows. Subgenital plates free from each other; articulated with valve; macrosetae scattered, irregularly arranged. Style broadly bilobed basally, median anterior lobe pronounced. Basal processes of the aedeagus/connective absent or reduced. Aedeagus with single shaft and gonopore; often asymmetrical. Connective anterior arms somewhat divergent, Y-shaped; articulated with or fused to aedeagus. 
Female genitalia. Pygofer with macrosetae reduced or absent or with numerous macrosetae. Ovipositor protruding far beyond pygofer apex or not. First valvula convex or not strongly convex, dorsal sculpturing pattern strigate, concatenate, reticulate, or with rectangular shaped cells; sculpturing reaching dorsal margin or submarginal; without distinctly delimited ventroapical sculpturing. Second valvula broad and gradually tapered apically, gradually broadened medially or subapically, or slender throughout; without dorsal median tooth; teeth on apical $1 / 3$ or more or restricted to apical $1 / 4$ or less; teeth large and regularly shaped or small, regularly or irregularly shaped.

\section{Geography and ecology}

Distribution: Palearctic, Oriental, and Nearctic regions. This tribe has an interesting disjunct distribution between the two subtribes, Koebeliina (western North America) and Grypotina (Oriental and Palearctic regions). Grypotes puncticollis (Herrich-Schäffer, 1834) is adventive in the northeastern United States (Wheeler 1989). All host records of Koebeliini are from Pinus spp. G. puncticollis feeds on P. sylvestris L. (Scotch pine) and has also been recorded from Swiss mountain pine, P. mugo Turra (Wheeler 1989) and P. nigricans Host.

\section{Remarks}

Koebeliini contains 6 genera and 15 species. Phylogenetic analyses strongly support its monophyly (Grypotellus and Koebelia included in analyses) and suggest (with little or no branch support) that the tribe may be related to Mukariini or Cochlorhinini, with which it shares the presence of platellae on metatarsomere I.

\section{Selected references}

Oman (1971), Dietrich \& Dmitriev (2003), Dmitriev (2003).

Included subtribes:

Grypotina Haupt, 1929

Fig. 30

Type genus: Grypotes Fieber, 1866.

\section{Diagnosis}

Grypotina are small to medium sized, yellow, greenish, or tawny leafhoppers. They can be distinguished from Koebeliina by the crown not or weakly produced; anterior margin of head shagreen, crown rounded to face, with transverse striations, or carinate, not foliaceous; forewing with appendix restricted to anal margin; forewing veins not pustulate; metafemur penultimate pair of macrosetae close-set, when present; metatibia dorsal rows with distinct intercalary setae; metatibia pecten without platellae; first valvula curved; first valvula dorsal sculpturing pattern imbricate, reaching dorsal margin; second valvula with teeth on apical $1 / 3$ or more.

\section{Description}

HeAD. Head subequal to or wider than pronotum. Discal portion of crown glabrous with radial or longitudinal striae or shagreen. Anterior margin of head shagreen, with numerous transverse striations, or with a single transverse carina; sometimes irregularly shaped. Frontoclypeus not tumid; texture shagreen. Clypellus narrow; parallel-sided or tapering apically; apex extending well beyond normal curve of gena. Lorum subequal to or wider than clypellus near base. Antennal bases near middle or posteroventral (lower) corners of eyes. Antennae short, less than $1.5 \mathrm{x}$ width of head or long, $1.5 \mathrm{x}$ 
width of head or longer. Gena obtusely incised laterally; with fine erect seta beside laterofrontal suture. Antennal ledges absent. Ocelli present; distant from eyes; on anterior margin of head.

THORAX. Pronotum lateral margin carinate; lateral margin shorter than basal width of eye.

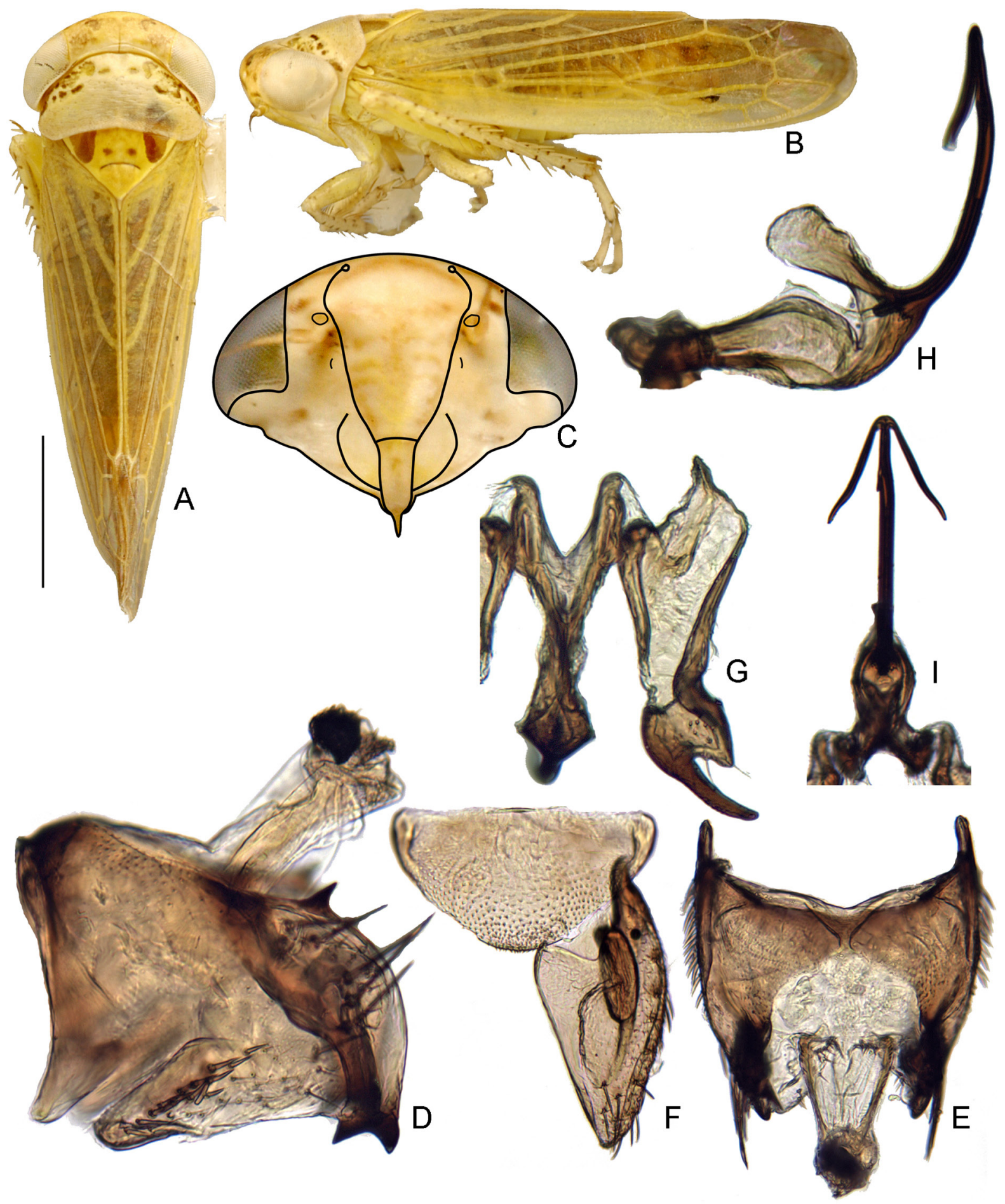

Fig. 30. Koebeliini (Grypotina). Grypotes puncticollis (Herrich-Schäffer, 1834). A-I. Standard views (see Material \& Methods). H-I. Aedeagus shown with partially fused connective at base. 
WINGS. Forewing macropterous; appendix restricted to anal margin; with 3 anteapical cells; veins not raised; without reflexed costal veins; A1-A2 crossvein absent; apical venation not highly reticulate.

LEGs. Profemur with AM1 seta only; intercalary row with one row of five or more fine setae; row AV with short, stout setae. Protibia dorsal surface rounded, convex. Metafemur apex macrosetae $2+2+1$. Metatarsomere I not expanded apically; plantar surface with one or more platellae.

MaLe Genitalia. Valve articulated with pygofer; with short point of articulation with pygofer. Pygofer basolateral membranous cleft present; macrosetae well differentiated into several rows. Subgenital plates free from each other; articulated with valve; macrosetae scattered, irregularly arranged. Style broadly bilobed basally, median anterior lobe pronounced. Basal processes of the aedeagus/connective absent. Aedeagus without basal hinge; with a single shaft and gonopore. Connective anterior arms somewhat divergent, $\mathbf{Y}$ - or $\mathbf{U}$-shaped; articulated with or fused to aedeagus.

Female genitalia. Pygofer with numerous macrosetae. Ovipositor not protruding far beyond pygofer apex. First valvula curved; dorsal sculpturing pattern concatenate, reticulate, or with rectangular shaped cells; sculpturing reaching dorsal margin; without distinctly delimited ventroapical sculpturing. Second valvula broad, gradually tapered; without dorsal median tooth; teeth on apical 1/3 or more; teeth large, regularly shaped or teeth small, regularly or irregularly shaped.

\section{Geography and ecology}

Distribution: Palearctic, Oriental and Nearctic (adventive) regions (Grypotes puncticollis is introduced in North America).

\section{Remarks}

Grypotina contains 5 genera and 10 species.

\section{Included genera}

Grypotellus Emeljanov, 1999

Grypotes Fieber, 1866

Pinopona Viraktamath \& Sohi, 1998

Shivapona Ghauri \& Viraktamath, 1987

Sohipona Ghauri \& Viraktamath, 1987

Koebeliina Baker, 1897

Fig. 31

Type genus: Koebelia Baker, 1897.

\section{Diagnosis}

Koebeliina are medium sized to large brown leafhoppers. They are distinguished from Grypotina by the crown strongly produced, spatulate, anterior margin of head foliaceous, forewing veins pustulate, appendix absent, metatibia dorsal rows without or with indistinct intercalary setae, metatibia pecten with platellae, first valvula relatively straight and not strongly curved, first valvula dorsal sculpturing pattern reticulate to maculose and submarginal, and second valvula with teeth restricted to apical 1/4.

\section{Description}

HEAD. Head subequal to or wider than pronotum. Discal portion of crown shagreen. Anterior margin of head foliaceous. Frontoclypeus not tumid; texture shagreen. Clypellus narrow; parallel-sided or tapering apically; apex extending well beyond normal curve of gena. Lorum subequal to or wider than clypellus 


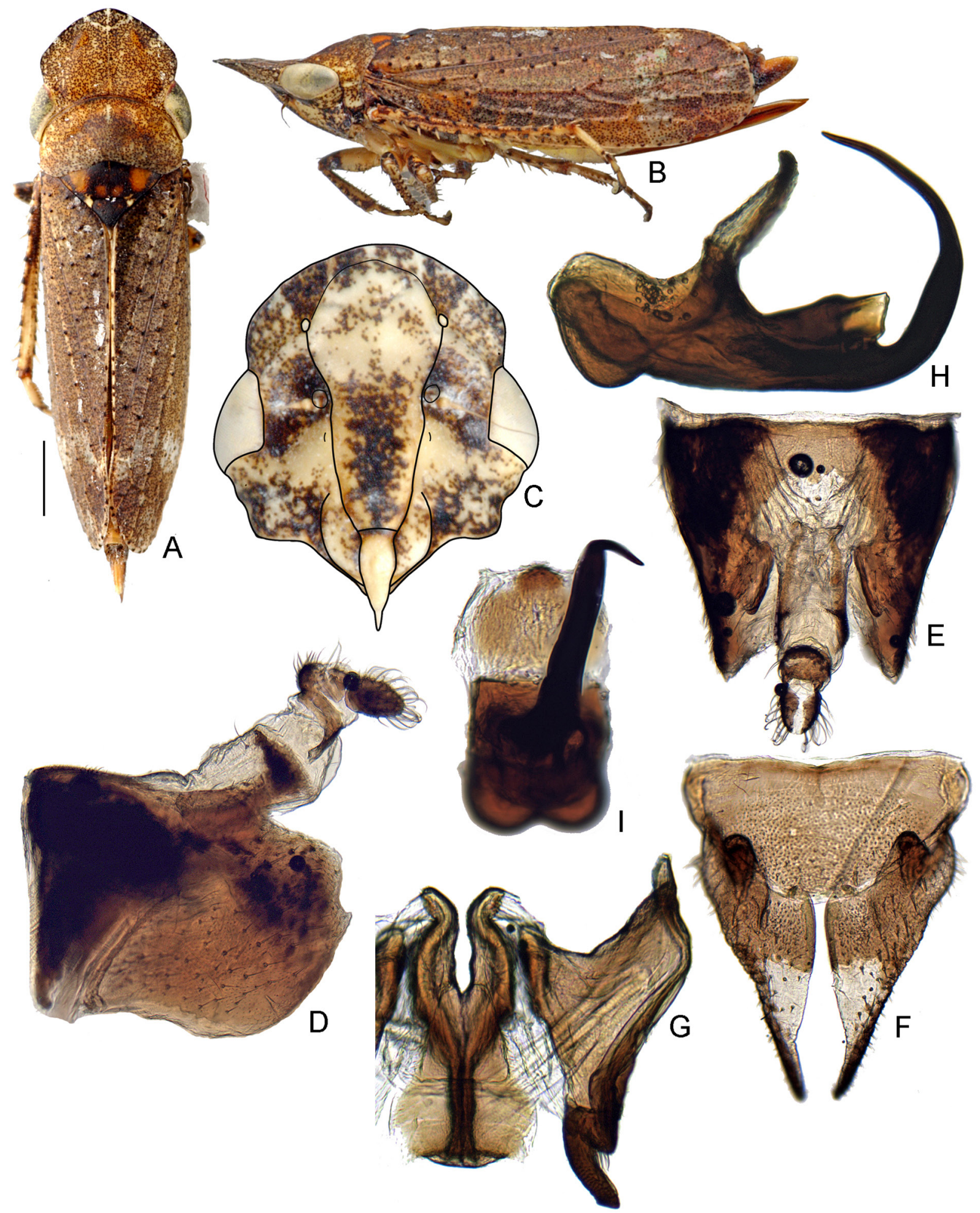

Fig. 31. Koebeliini (Koebeliina). Koebelia grossa Ball, 1909. A-I. Standard views (see Material \& Methods). 
near base. Antennal bases near middle or posteroventral (lower) corners of eyes. Antennae short, less than $1.5 \mathrm{x}$ width of head. Gena obtusely incised laterally; with fine erect seta beside laterofrontal suture. Antennal ledges absent. Ocelli present; distant from eyes; on face.

THORAx. Pronotum lateral margin not carinate; lateral margin shorter than basal width of eye.

WiNGS. Forewing macropterous or submacropterous; appendix absent or reduced; with 3 anteapical cells; veins not raised or slightly raised; veins pustulate; without reflexed costal veins; A1-A2 crossvein absent; apical venation not highly reticulate.

LEgs. Profemur with AM1 seta only; intercalary row reduced or absent; row AV with relatively long macrosetae. Protibia dorsal surface flat, $\mathrm{AD}$ and $\mathrm{PD}$ margins at $\sim 90^{\circ}$ angles but not carinate. Metafemur apex macrosetae $2+1$ or $2+2$. Metatarsomere I not expanded apically; plantar surface with one or more platellae.

Male Genitalia. Valve articulated with pygofer; with short point of articulation with pygofer. Pygofer basolateral membranous cleft present; macrosetae absent or reduced ( $\leq$ two rows). Subgenital plates free from each other, articulated with valve; macrosetae scattered, irregularly arranged. Style broadly bilobed basally, median anterior lobe pronounced. Basal processes of the aedeagus/connective absent. Aedeagus with large apical process arising from ventral margin; without basal hinge; with a single shaft and gonopore. Connective anterior arms somewhat divergent, Y-shaped; articulated with aedeagus.

Female Genitalia. Pygofer with macrosetae reduced or absent. Ovipositor protruding or not protruding far beyond pygofer apex. First valvula not strongly convex; dorsal sculpturing pattern reticulate or maculose; sculpturing reaching dorsal margin or submarginal; without distinctly delimited ventroapical sculpturing. Second valvula broad, gradually tapered or slender throughout; without dorsal median tooth; teeth restricted to apical $1 / 4$ or less; teeth small, regularly or irregularly shaped.

\section{Geography and ecology}

Distribution: Nearctic (Western North America).

\section{Remarks}

Koebeliina contains the type genus and 5 species.

\section{Included genera}

Koebelia Baker, 1897

\section{Limotettigini Baker, 1915}

Fig. 32

Type genus: Limotettix Sahlberg, 1871.

\section{Diagnosis}

Limotettigini are small to medium sized ivory, greyish, or black leafhoppers, often with dark markings. They can be identified by the parallel-sided or tapering clypellus, pygofer with a spine-like process arising from the dorsal margin, and aedeagus articulated with a plate-like "dorsal connective" at the dorsal margin of the socle. 


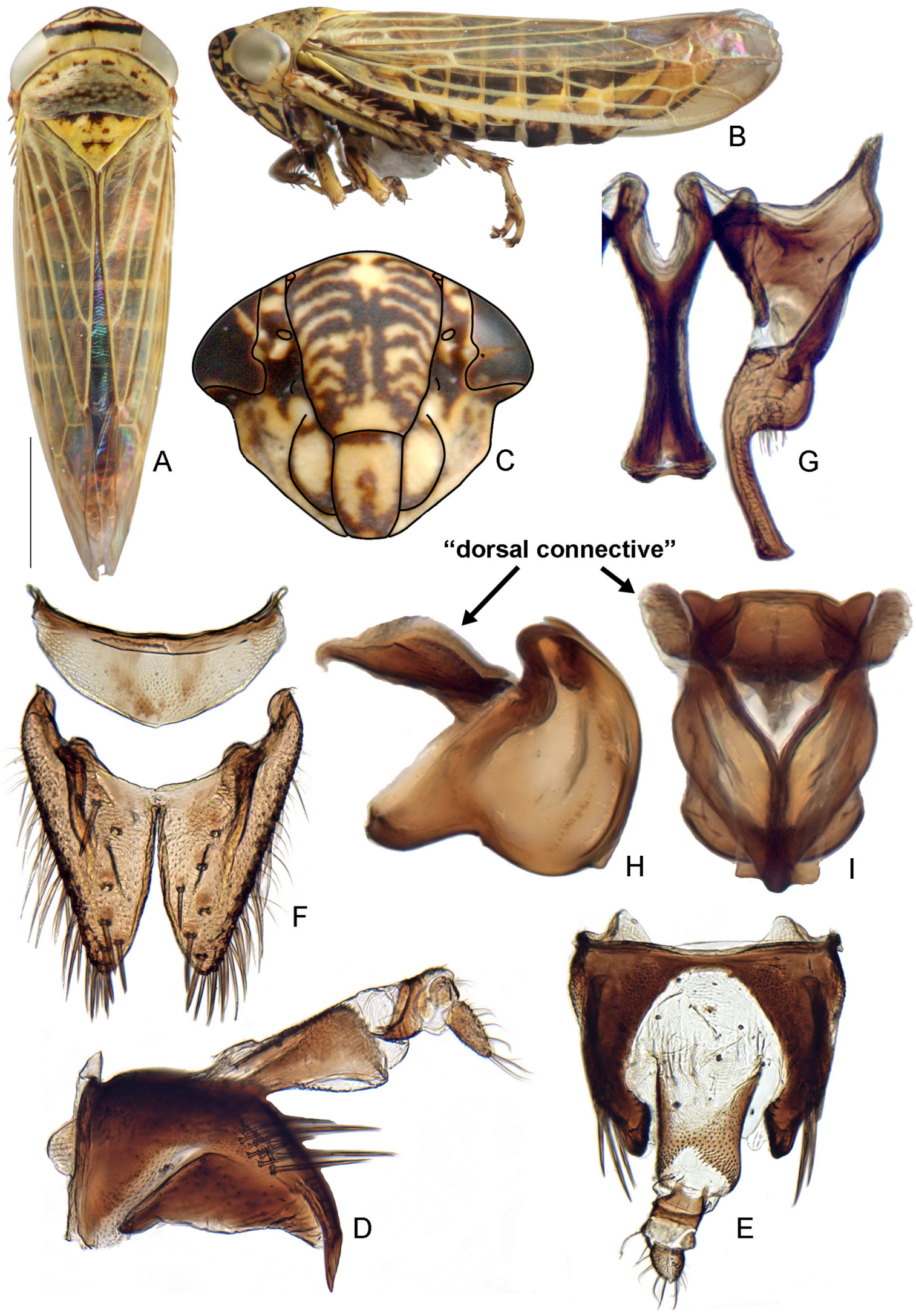

Fig. 32. Limotettigini. A-B, D-I. Limotettix striolus (Fallén, 1906). C. L. decumanus Kontkanen, 1949. A-I. Standard views (see Material \& Methods). F. valve shown dissociated from plates. 


\section{Description}

HEAD. Head subequal to or wider than pronotum. Discal portion of crown glabrous with radial or longitudinal striae or completely shagreen to base. Anterior margin of head shagreen. Frontoclypeus not tumid; texture shagreen. Clypellus parallel-sided or tapering apically; apex following or slightly surpassing normal curve of gena. Lorum distinctly narrower than clypellus near base. Antennal bases near middle or posteroventral (lower) corners of eyes. Antennae short, less than $1.5 \mathrm{x}$ width of head. Gena obtusely incised laterally; with fine erect seta beside laterofrontal suture. Antennal ledges absent. Ocelli present; close to eyes; on anterior margin of head.

Thorax. Pronotum lateral margin carinate; lateral margin shorter than basal width of eye.

WiNGS. Forewing macropterous; appendix restricted to anal margin; with 3 anteapical cells; veins not raised; without reflexed costal veins; A1-A2 crossvein absent; apical venation not highly reticulate.

LEgs. Profemur with AM1 seta only; intercalary row with one row of five or more fine setae; row AV with short, stout setae. Protibia dorsal surface rounded, convex. Metafemur apex macrosetae $2+2+1$. Metatarsomere I not expanded apically; plantar setae simple, tapered.

Male Genitalia. Valve articulated with pygofer; with short point of articulation with pygofer. Pygofer basolateral membranous cleft present; macrosetae well differentiated into several rows; with spinelike process arising from dorsal side. Subgenital plates free from each other, articulated with valve; macrosetae uniseriate laterally or with two lateral rows of macrosetae, sometimes with some irregularly arranged macrosetae. Style broadly bilobed basally, median anterior lobe pronounced. Basal processes of the aedeagus/connective absent or reduced. Aedeagus without basal hinge; with a single shaft and gonopore; with plate-like "dorsal connective" articulated to dorsal margin of socle. Connective anterior arms somewhat divergent, Y-shaped; articulated with aedeagus.

Female genitalia. Pygofer with numerous macrosetae. Ovipositor not protruding far beyond pygofer apex. First valvula not strongly convex; dorsal sculpturing pattern imbricate (with overlapping scales); sculpturing reaching dorsal margin; without distinctly delimited ventroapical sculpturing. Second valvula broad, gradually tapered; without dorsal median tooth; teeth on apical 1/3 or more; teeth small, regularly or irregularly shaped.

\section{Geography and ecology}

Distribution: cosmopolitan. Some species are found around wetlands, bogs, and peatlands, while others are found in grasslands or transitional habitats. Host plant families include Juncaceae, Cyperaceae, Poaceae, Myricaceae, Ericaceae, and Asteraceae. L. (Scleroracus) flavopictus (Ishihara, 1953) is a vector of Japanese aster yellows and potato witches' broom in Japan and L. (Scleroracus) vaccinii (Van Duzee, 1890) is a vector of false blossom of cranberries in eastern North America.

\section{Remarks}

Limotettigini contains the type genus with 5 subgenera and 89 species, following the classification of Hamilton (1994). In the phylogenetic analyses, Limotettix was consistently placed within the large grass feeding clade of Deltocephalinae, but its position in the clade is uncertain. It may be related to Cicadulini, the Athysanus-group (Athysanini), Hecalini, or Macrostelini. Dmitriev (2000, 2002) included Anoterostemmina as a subtribe of Limotettigini but a relationship between these groups was not supported in the phylogenetic analyses here, wherein molecular data for Anoterostemma were included for the first time. Instead, Anoterostemma is resolved near Euscelis and other Athysanus-group genera, and it is transferred to Athysanini here. 


\section{Selected references}

Hamilton (1994), McKamey (2001).

\section{Included genera}

Limotettix Sahlberg, 1871

\section{Luheriini Linnavuori, 1959}

Fig. 33

Type genus: Luheria Osborn, 1923.

\section{Diagnosis}

Luheriini are medium sized to large yellow, orange, and brown leafhoppers. They can be identified by the anterior margin of the head with numerous striations or carinae, forewing appendix absent or reduced, forewings not overlapping, central anteapical cell constricted medially, T-shaped connective, and 'linear' style.

\section{Description}

HeAd. Head wider than pronotum. Discal portion of crown glabrous with radial or longitudinal striae. Anterior margin of head with numerous transverse striations or carinae. Frontoclypeus not tumid; texture shagreen. Clypellus widening apically; apex following or slightly surpassing normal curve of gena. Lorum subequal to or wider than clypellus near base. Antennal bases near middle or posteroventral (lower) corners of eyes. Antennae moderately long, about as long as width of head. Gena obtusely incised laterally; with fine erect seta beside laterofrontal suture. Antennal ledges weakly developed (carinate or weakly carinate). Ocelli present; close to eyes; on anterior margin of head.

Thorax. Pronotum lateral margin carinate; lateral margin shorter than basal width of eye.

WiNGS. Forewing macropterous; appendix absent or reduced; commissural margin straight; with 3 anteapical cells; central anteapical cell constricted medially; veins not raised; with or without reflexed costal veins; A1-A2 crossvein present.

Legs. Profemur with AM1 and with one or more additional proximal setae; intercalary row with one row of five or more fine setae; row AV with short, stout setae. Protibia dorsal surface rounded, convex. Metafemur apex macrosetae $2+2+1$. Metatarsomere I not expanded apically; plantar setae simple, tapered.

Male genitalia. Valve articulated with pygofer; lateral margin short, articulating with pygofer at a point. Pygofer basolateral membranous cleft present; macrosetae well differentiated into several rows. Subgenital plates free from each other; articulated with valve; macrosetae scattered, irregularly arranged. Style linear, median anterior lobe not pronounced. Basal processes of the aedeagus/connective absent. Aedeagus with single shaft and gonopore. Connective anterior arms widely divergent, $\mathbf{T}$-shaped; articulated with aedeagus. Segment X large; well sclerotized dorsally; with anteroventral processes.

Female genitalia. Pygofer with numerous macrosetae. Ovipositor not protruding far beyond pygofer apex. First valvula convex, dorsal sculpturing pattern strigate; sculpturing reaching dorsal margin; without distinctly delimited ventroapical sculpturing. Second valvula relatively slender, gradually tapered; without dorsal median tooth; teeth restricted to apical $1 / 4$ or less; teeth small, regularly or irregularly shaped. 


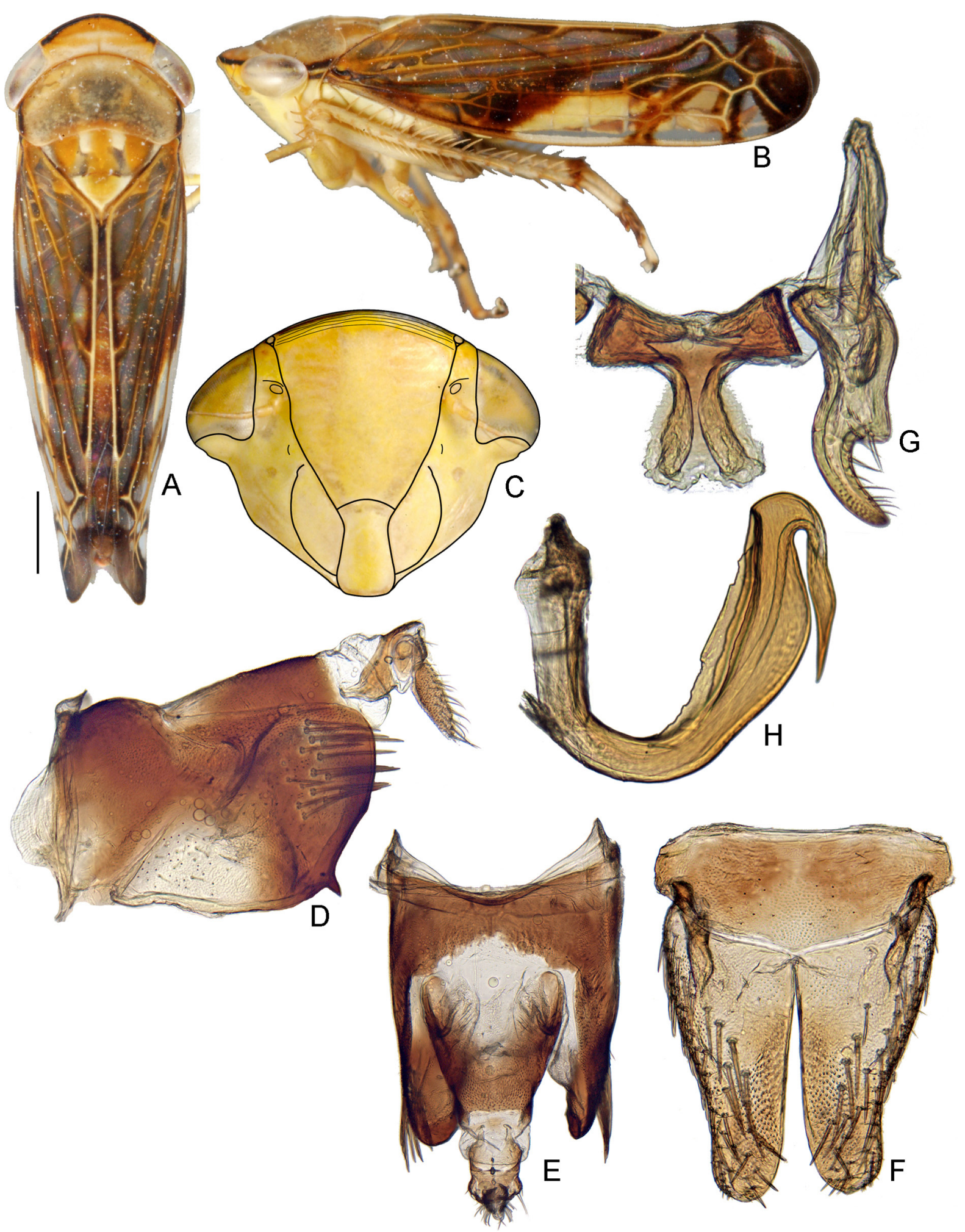

Fig. 33. Luheriini. Luheria constricta Osborn, 1923. A-I. Standard views (see Material \& Methods). 


\section{Geography and ecology}

Distribution: Neotropical. The only known species, Luheria constricta Osborn, 1923, is widely distributed in South America from northern Argentina to Bolivia and northeastern Brazil. Host plants are unknown. Males exhibiting mud-puddling behavior have been collected on wet soil.

\section{Remarks}

The tribe contains only the type genus and species. Phylogenetic analyses and some plesiomorphic characters of the head, wings, and male genitalia show that this is an early diverging lineage of Deltocephalinae. It appears to be most closely related to the Old World tribe Fieberiellini. L. constricta has an unusual 28S rDNA sequence that contains several large insertions, and some regions were impossible to amplify using primers that worked well for most other Deltocephalinae. This divergent sequence resulted in its long terminal branch in phylogenetic analyses.

\section{Selected references}

Linnavuori (1959), Zahniser (2005).

\section{Included genera}

Luheria Osborn, 1923

\section{Macrostelini Kirkaldy, 1906}

Fig. 34

Type genus: Macrosteles Fieber, 1866.

$=$ Balcluthini Baker, 1915 .

$=$ Gnathodini Baker, 1915 .

$=$ Coryphaelini Nast, 1972 .

\section{Diagnosis}

Macrostelini are small to medium sized, slender, often stramineous, yellow, or greenish leafhoppers, with or without dark markings. They can be identified by their long, slender shape, forewing with 2 anteapical cells, subgenital plates usually with membranous digitate apical lobe, and male pygofer macrosetae sometimes plumose.

\section{Description}

HEAD. Head subequal to or wider than pronotum or distinctly narrower than pronotum. Discal portion of crown glabrous with radial or longitudinal striae or shagreen. Anterior margin of head shagreen. Frontoclypeus not tumid; texture shagreen. Clypellus parallel-sided or tapering apically; apex following or slightly surpassing normal curve of gena. Lorum subequal to, wider than, or distinctly narrower than clypellus near base. Antennal bases near middle or posteroventral (lower) corners of eyes. Antennae short, less than $1.5 \mathrm{x}$ width of head. Gena obtusely incised laterally; with fine erect seta beside laterofrontal suture. Antennal ledges absent. Ocelli present; close to or distant from eyes; on anterior margin of head.

THORAX. Pronotum lateral margin carinate or not; lateral margin shorter, as long as, or longer than basal width of eye.

WINGS. Forewing macropterous or submacropterous; appendix restricted to anal margin; with 2 anteapical cells; veins not raised; without reflexed costal veins; A1-A2 crossvein absent; apical venation not highly reticulate. 


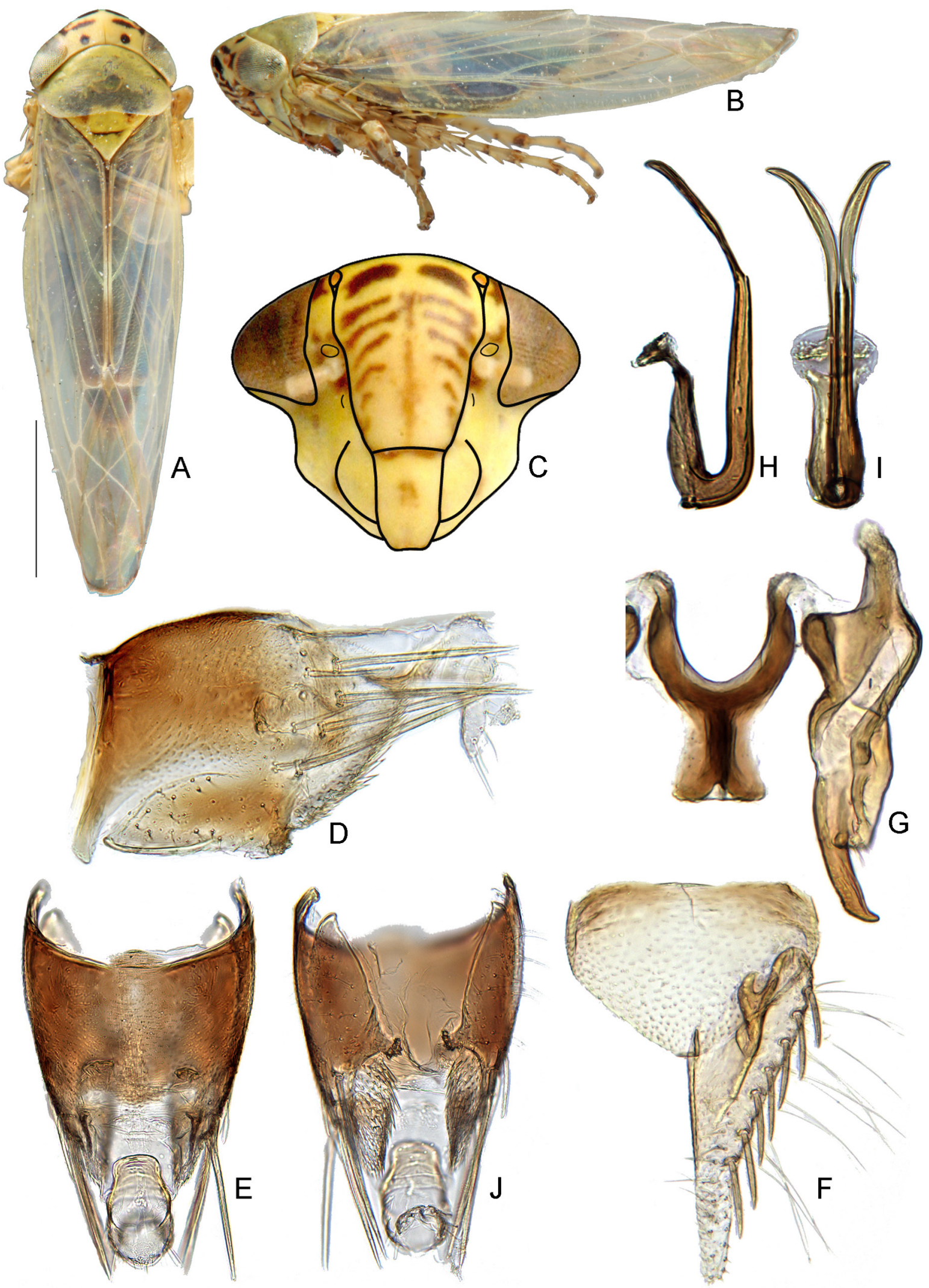

Fig. 34. Macrostelini. Macrosteles quadrilineatus (Forbes, 1885). A-I. Standard views (see Material \& Methods). J. Ventral view of pygofer. 
Legs. Profemur with AM1 seta only; intercalary row with one row of five or more fine setae; row AV without setae, with thin hair-like setae, or with short, stout setae. Protibia dorsal surface rounded, convex. Metafemur apex macrosetae $2+1+1$ or $2+2+1$. Metatarsomere I not expanded apically; plantar setae simple, tapered.

MaLe Genitalia. Valve articulated with pygofer; lateral margin short, articulating with pygofer at a point. Pygofer basolateral membranous cleft present; macrosetae absent or reduced ( $\leq$ two rows) or well differentiated into several rows; macrosetae sometimes plumose. Subgenital plates free from each other; articulated with valve; macrosetae uniseriate laterally. Style broadly bilobed basally, median anterior lobe pronounced. Basal processes of the aedeagus/connective absent or reduced. Aedeagus with single shaft and gonopore. Connective anterior arms somewhat divergent, $\mathbf{Y}$ - or $\mathbf{U}$-shaped; articulated with aedeagus.

Female genitalia. Pygofer with numerous macrosetae. Ovipositor not protruding far beyond pygofer apex. First valvula convex or not strongly convex; dorsal sculpturing pattern granulose, maculose, or imbricate (with overlapping scales); sculpturing reaching dorsal margin or submarginal; without distinctly delimited ventroapical sculpturing or with distinctly delimited apicoventral sculpturing. Second valvula abruptly broadened medially or subapically, or broad, gradually tapered, or gradually broadened medially or subapically; without dorsal median tooth; if present, teeth on apical 1/3 or more; teeth absent or small, regularly or irregularly shaped.

\section{Geography and ecology}

Distribution: cosmopolitan. Some genera (e.g., Balclutha, Dalbulus) feed mostly on grasses or sedges, while others (e.g., Macrosteles) feed on a wide variety of hosts including herbaceous dicots, grasses, and sedges. Macrosteles frontalis (Scott, 1875) lives on horsetail (Equisetum sp.), a primitive vascular plant. This tribe includes a number of economically important vectors of plant pathogens. Dalbulus maidis (DeLong, 1923), the corn leafhopper, and D. elimatus (Ball, 1900) are especially damaging to maize as vectors of corn stunt spiroplasma, maize bushy stunt phytoplasma, and maize rayado fino virus in Mexico and Central and South America (Nault \& Ammar 1989). Macrosteles quadrilineatus (Forbes, 1885), the aster leafhopper, transmits American and European aster yellows to numerous plants. Other species of Macrosteles transmit clover phyllody, clover dwarf, aster yellows, stolbur, Koksaghyz yellows, chrysanthemum yellows, lissers, anemone witches' broom, eggplant dwarf, garland chrysanthemum witches' broom, marguerite yellows, mitsuba witches' broom, onion yellows, tomato yellows, white leaf phytoplasma, little cherry, and oat blue dwarf. Cicadulina mbila (Naudé, 1924) and congeneric species transmit maize streak in sub-Saharan Africa.

\section{Remarks}

Macrostelini contains 37 genera and 316 species. A morphological phylogenetic analysis (Knight \& Webb 1993) which included exemplars of all genera resolved the tribe as monophyletic. Three of the four exemplars in phylogenetic analyses here (Macrosteles, Dalbulus, and Balclutha) were resolved as a monophyletic group with strong branch support. This monophyletic group is included in the large grassfeeding clade but its relationship to other tribes in the clade is not clear. The fourth exemplar included in analyses here is Evinus which was recently placed in the tribe (Lu et al. 2011); it was resolved near the grass feeding tribes Chiasmini, Stenometopiini, and Eupelicini but branch support was not strong and its exact relationship to other Deltocephalinae needs further study. Like other Macrostelini, it has two anteapical cells in the forewing. 


\section{Selected references}

Blocker (1967), Knight (1987), Knight \& Webb (1993), Webb \& Vilbaste (1994), Dietrich et al. (1998), Dmitriev (2003).

\section{Included genera}

Aderganna Knight \& Webb, 1993

Afrosteles Theron, 1975

Agelina Oman, 1938

Alebranus Linnavuori, 1959

Argaterma White, 1878

Atlantocella Dlabola, 1982

Balclutha Kirkaldy, 1900

Baldulus Oman, 1934

Balolina Knight \& Webb, 1988

Cicadabara Knight \& Webb, 1993

Cicadulella China, 1928

Cicadulina China, 1926

Cicaduloida Osborn, 1934

Cortona Oman, 1938

Coryphaelus Puton, 1886

Dalbulus DeLong, 1950

Davisonia Dorst, 1937

Elrabonia Linnavuori, 1959

Evinus Dlabola, 1977

Kadrabolina Knight \& Webb, 1993

Macrosteles Fieber, 1866

Marquesitettix Metcalf, 1952

Masafuera Knight \& Webb, 1993

Nesoclutha Evans, 1947

Nesolina Osborn, 1935

Nesophyla Osborn, 1934

Nesoriella Osborn, 1934

Nyhimbricus Webb, 1986

Paracicadula Osborn, 1934

Sagatus Ribaut, 1948

Sanctahelenia Dlabola, 1976

Scaphoidulina Osborn, 1934

Scaphosteles Knight \& Webb, 1993

Sonronius Dorst, 1937

Stellena Theron, 1973

Teyasteles Linnavuori, 1969

Yamatotettix Matsumura, 1914

Magnentiini Linnavuori, 1978

Fig. 35

Type genus: Magnentius Singh-Pruthi, 1930. 


\section{Diagnosis}

Magnentiini are medium sized black, coarsely punctate, wedge-shaped leafhoppers. They can be identified by their robust appearance, coarsely punctate texture of the pronotum and face, hirsute body and face, crown very short, pronotum produced anteriorly with apex exceeding eyes in dorsal view, ocelli distant from eyes, antennal ledge distinct, forewing appendix large, and forewing veins raised.

\section{Description}

HeAd. Head subequal to or wider than pronotum. Discal portion of crown punctate. Anterior margin of head punctate. Frontoclypeus not tumid or tumid, texture punctate. Clypellus parallel-sided, tapering apically, or widening apically; apex following or slightly surpassing normal curve of gena. Lorum subequal to or wider than clypellus near base. Antennal bases near middle or posteroventral (lower) corners of eyes. Antennae short, less than $1.5 \mathrm{x}$ width of head. Gena not or slightly incised laterally; hirsute, with many fine hairs (difficult to interpret presence/absence of fine erect seta). Antennal ledges strongly developed, with distinct ledge. Ocelli present; distant from eyes; on face.

THorax. Pronotum exceeding eyes anteriorly; lateral margin carinate; lateral margin shorter than basal width of eye.

WINGS. Forewing macropterous or submacropterous; appendix present, extending around wing apex; with 3 anteapical cells; veins distinctly raised; without reflexed costal veins; A1-A2 crossvein present; apical venation not highly reticulate.

Legs. Profemur with AM1 seta only; intercalary row with one row of five or more fine setae; row AV with relatively long macrosetae. Protibia dorsal surface rounded, convex. Metafemur apex macrosetae $2+0$. Metatarsomere I not expanded apically; plantar setae simple, tapered.

Male genitalia. Valve articulated with pygofer; lateral margin short, articulating with pygofer at a point. Pygofer dorsoapical margin incised to near mid-length; basolateral membranous cleft present; macrosetae well differentiated into several rows. Subgenital plates free from each other; articulated with valve; macrosetae scattered, irregularly arranged. Style broadly bilobed basally, median anterior lobe pronounced. Basal processes of the aedeagus/connective absent. Aedeagus with single shaft and gonopore. Connective anterior arms somewhat divergent, Y-shaped; articulated with aedeagus.

Female genitalia. Pygofer with macrosetae reduced or absent. Ovipositor not protruding far beyond pygofer apex. First valvula not strongly convex; dorsal sculpturing pattern strigate; sculpturing reaching dorsal margin; without distinctly delimited ventroapical sculpturing. Second valvula abruptly broadened medially or subapically; without dorsal median tooth; teeth on apical $1 / 3$ or more; teeth small, regularly or irregularly shaped.

\section{Geography and ecology}

Distribution: Afrotropical and Oriental regions.

\section{Remarks}

Magnentiini contains 2 genera and 4 species. Although it bears a strong superficial resemblance to Nionia, it was transferred from Nioniinae to Deltocephalinae by Zahniser \& Dietrich (2010) based on the deltocephaline-like male genitalia.

Magnentius was included here for the first time in analyses with molecular data. The placement in Deltocephalinae was supported in analyses here as it was resolved with moderate to strong branch support (MP BS=71, DI=7) as sister to three Penthimiini in MP analyses and sister to two Penthimiini 


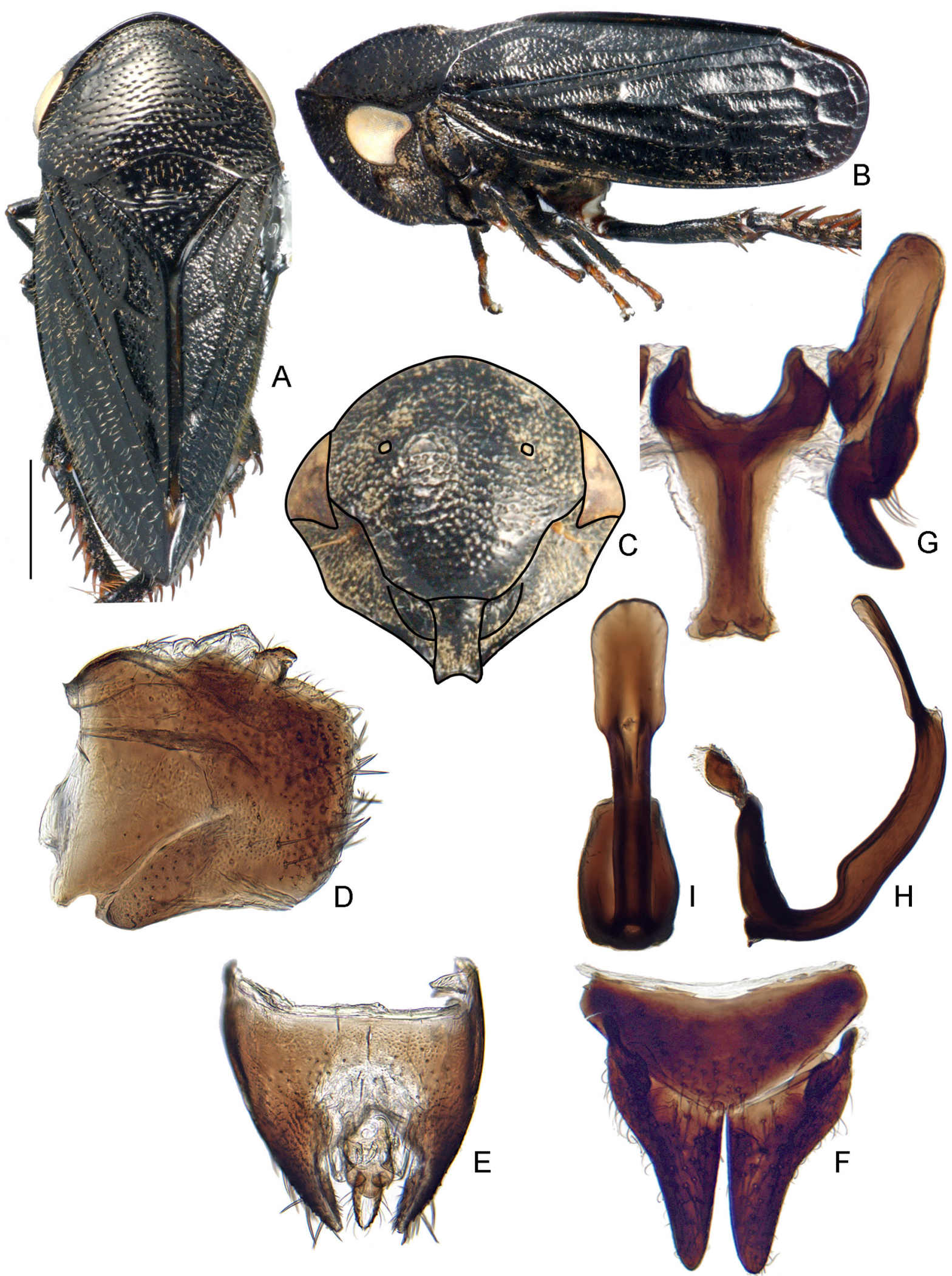

Fig. 35. Magnentiini. Magnentius clavatus Singh-Pruthi, 1930. A-I. Standard views (see Material \& Methods). 
in $\mathrm{ML}$ and Bayesian analyses with strong support ( $\mathrm{ML} B S=93, \mathrm{PP}=1.0$, respectively). Some characters that are unusual among Deltocephalinae, including its robust appearance, ocelli distant from the eyes, strong antennal ledges, and forewing appendix extending around the wing apex, support its relationship to Penthimiini.

\section{Selected references}

Linnavuori (1978a).

\section{Included genera}

Magnentius Singh-Pruthi, 1930

Ndua Linnavuori, 1978

Mukariini Distant, 1908

Fig. 36

Type genus: Mukaria Distant, 1908.

\section{Diagnosis}

Mukariini are small to medium sized, often dorsoventrally depressed or ventrally flattened, brown, black, whitish, yellow, or green, leafhoppers, sometimes marked with orange or red. They can be identified by the produced head, often with the frontoclypeus tumid distally, ventral part of face flat and lying nearly horizontally or concave, and ocelli distant from eyes. Other distinctive characters (e.g. bifurcate aedeagus with 2 gonopores in Mukaria and Pseudobalbillus) exist within the group but are not shared by all taxa.

\section{Description}

HeAD. Head often somewhat produced; subequal to or wider than pronotum. Discal portion of crown glabrous with radial or longitudinal striae. Anterior margin of head shagreen, striate, or with one to several carinae. Frontoclypeus often tumid distally; texture shagreen, glabrous, or striate. Ventral part of face lying in nearly horizontal plane, or concave. Clypellus widening apically; apex following or slightly surpassing normal curve of gena. Lorum subequal to, wider than, or distinctly narrower than clypellus near base. Antennal bases near upper or anterodorsal corners of eyes. Antennae short, less than $1.5 \mathrm{x}$ width of head, or long. Gena obtusely incised laterally; with fine erect seta beside laterofrontal suture. Antennal ledges strongly developed (with a definite ledge), or reduced or absent. Ocelli present; distant from eyes; on anterior margin of head.

Thorax. Pronotum not exceeding eyes anteriorly, lateral margin carinate, lateral margin shorter than basal width of eye.

WINGS. Forewing macropterous; appendix absent, reduced, or restricted to anal margin; with 2 or 3 anteapical cells; outer anteapical cell sometimes very close to or confluent with costal vein; veins not raised; without reflexed costal veins; A1-A2 crossvein absent; apical venation not highly reticulate.

Legs. Profemur with AM1 seta only; intercalary row with one row of five or more fine setae; row AV with thin, hair-like setae or without setae. Protibia dorsal surface rounded, convex. Metafemur apex macrosetae $2+2+1$. Metatarsomere I not expanded apically; plantar setae simple, tapered.

Male Genitalia. Valve articulated with pygofer; lateral margin short, articulating with pygofer at a point. Pygofer basolateral membranous cleft present; macrosetae absent or reduced ( $\leq$ two rows) or well differentiated into several rows; often with processes arising ventrally, apically, or medially. 


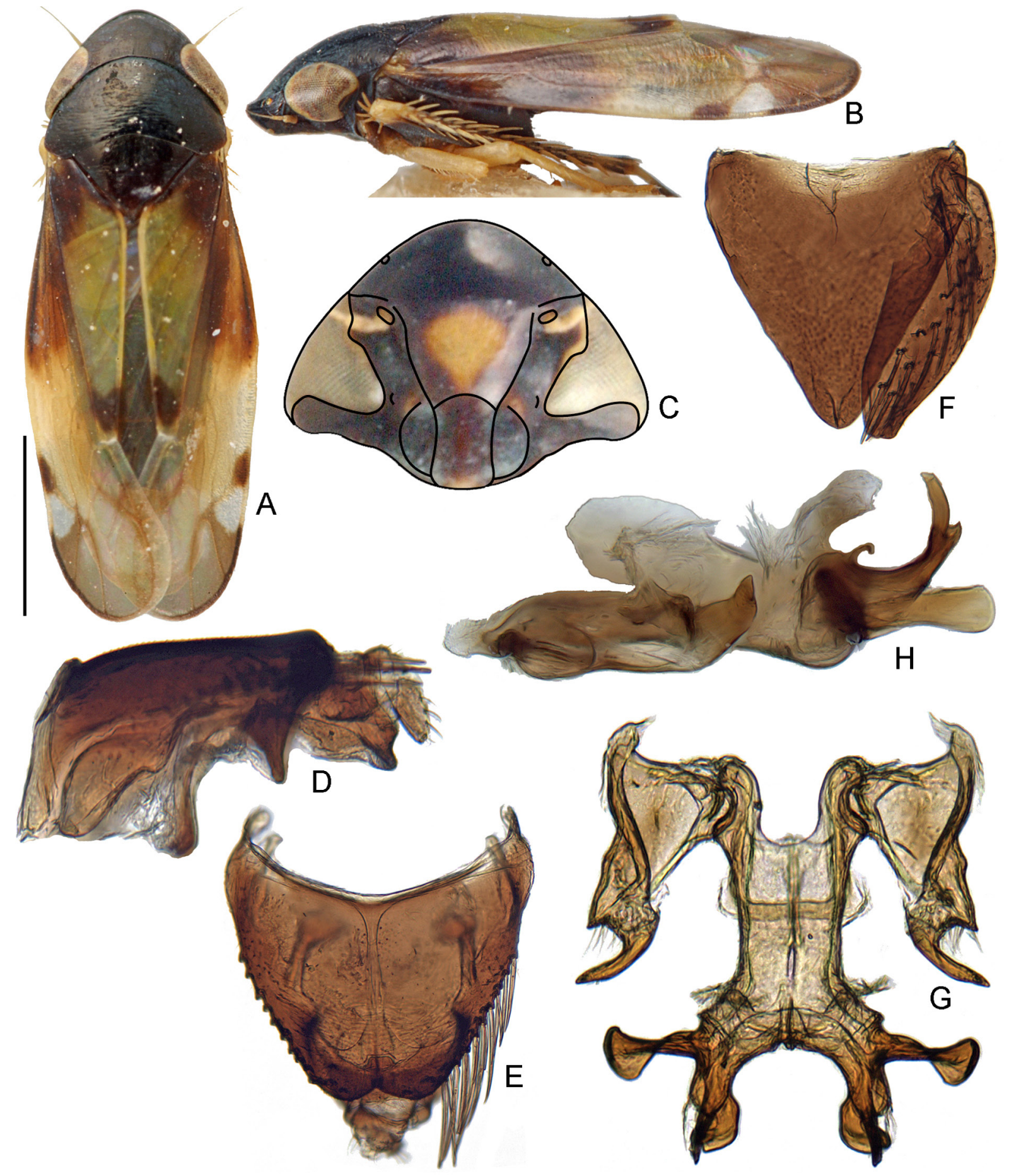

Fig. 36. Mukariini. Mukaria maculata (Matsumura, 1912). A-H. Standard views (see Material \& Methods). G. Connective and styles shown with aedeagus fused to connective. H. Aedeagus shown with connective and style. 
Subgenital plates free from each other; articulated with or rarely fused (Agrica) to valve; macrosetae scattered, irregularly arranged, or uniseriate laterally. Style broadly bilobed basally, median anterior lobe pronounced. Basal processes of the aedeagus/connective absent or reduced or present, connected or articulated to connective or near base of aedeagus. Aedeagus with single shaft and gonopore or shaft divided near base, with two gonopores. Connective anterior arms somewhat divergent, $\mathbf{Y}$ - or $\mathbf{V}$-shaped; anterior arms sometimes very widely divergent; articulated with or fused to aedeagus.

Female genitalia. Pygofer with numerous macrosetae. Ovipositor not protruding far beyond pygofer apex. First valvula convex or not strongly convex; dorsal sculpturing pattern strigate; sculpturing reaching dorsal margin; without distinctly delimited ventroapical sculpturing. Second valvula broad, gradually tapered; without dorsal median tooth; teeth on apical 1/3 or more; teeth large, regularly shaped or small, regularly or irregularly shaped.

\section{Geography and ecology}

Distribution: Palearctic, Afrotropical, and Oriental regions. All known hosts are bamboos.

\section{Remarks}

Mukariini contains 12 genera and 54 species. The taxonomy of Mukariini has recently undergone some modification. It previously only contained Mukaria, Neobassareus, and Pseudobalbillus, but recently Chen et al. (2007) placed the Mohunia group in this tribe, Zahniser \& Dietrich (2010) included Scaphotettix and Agrica, and several new genera and species have been described (Hayashi, 1996; Chen et al. 2007, 2008, 2009; Li et al. 2007; Dai et al. 2009). The phylogenetic analyses here included representatives of Agrica, Mukaria, Scaphotettix, and an undescribed genus from China. Agrica and the undescribed genus were resolved as sister to each other, as were Mukaria and Scaphotettix, but the four were not resolved as monophyletic. However there is little or no support on the branches separating them, and it is expected that more data will resolve the group as monophyletic in future analyses. Some morphological characters and the restricted use of bamboos as hosts support the recognition of the group. The tribe is potentially related to Vartini, Koebeliini, or Cochlorhinini. Future phylogenetic studies on the group should also include representatives of the large African genus Pseudobalbillus to confirm its placement in Mukariini, other genera of which are found in the Oriental region.

\section{Selected references}

Linnavuori (1979b), Hayashi (1996), Li \& Chen (1998), Knight \& Webb (2002), Chen et al. (2007, 2008, 2009), Dai et al. (2009), Zahniser \& Dietrich (2010), Khatri \& Webb (2011), Yang \& Chen (2011).

\section{Included genera}

Agrica Strand, 1942

Benglebra Mahmood \& Ahmed, 1969

Flatfronta Chen \& Li, 1997

Mohunia Distant, 1908

Mukaria Distant, 1908

Neobassareus Kocak, 1981

Neomohunia Chen \& Li, 2007

Paramohunia Chen \& Li, 2007

Pseudobalbillus Jacobi, 1912

Pseudomohunia Li, Chen \& Zhang, 2007

Scaphotettix Matsumura, 1914

Tiaobeinia Chen \& Li, 2008 


\section{Occinirvanini Evans, 1966}

Fig. 37

Type genus: Occinirvana Evans, 1941.

\section{Diagnosis}

Occinirvanini are medium sized, elongate, bizarrely shaped, ivory and orange colored leafhoppers, with some fuscous coloration on the forewing apex. They can be identified by the elongate head, ocelli on the crown, ocelli very distant from eyes, foliaceous anterior margin of the head, very long antennae, antennal bases situated very high on the face anterad of the eyes, face strongly concave, and metatarsomere I plantar surface with platellae.

\section{Description}

HEAD. Head subequal to or wider than pronotum; strongly produced, elongate. Discal portion of crown shagreen. Anterior margin of head foliaceous. Face strongly concave. Frontoclypeus not tumid; texture shagreen. Clypellus widening apically; apex following or slightly surpassing normal curve of gena. Lorum subequal to or wider than clypellus near base. Antennal bases near upper part of face, anterad of eyes. Antennae very long, 3-4 x width of head or longer; scape and pedicel large. Gena obtusely incised laterally; hirsute, with many fine hairs (difficult to interpret presence/absence of fine erect seta). Antennal ledges absent. Ocelli present; distant from eyes, closer to crown apex than to adjacent eye; on crown.

THORAX. Pronotum not exceeding eyes anteriorly, lateral margin not carinate; lateral margin longer than basal width of eye.

WINGS. Forewing macropterous; appendix restricted to anal margin; with 3 anteapical cells; veins not raised; without reflexed costal veins; A1-A2 crossvein absent; apical venation not highly reticulate.

Legs. Profemur short, stout; with AM1 seta only; intercalary row with one row of five or more fine setae; row AV without setae or with few short, stout setae. Protibia dorsal surface flat, AD and PD margins at $\sim 90^{\circ}$ angles but not carinate. Metafemur apex macrosetae $2+2+1$. Metatarsomere I not expanded apically; plantar surface with one or more platellae.

MALE Genitalia. Valve articulated with pygofer; lateral margin short, articulating with pygofer at a point. Pygofer basolateral membranous cleft present; macrosetae well differentiated into several rows; without dorsal or ventral processes; subrectangular in shape. Subgenital plates free from each other, articulated with valve; with single row of macrosetae distant from lateral margin; apex with $\sim 5-7$ spaced macrosetae; lateral margin with numerous short thin or thick setae. Style broadly bilobed basally, median anterior lobe pronounced; apophysis with subapical dorsal tooth. Basal processes of the aedeagus/connective absent. Aedeagus with single shaft and gonopore; with "dorsal connective" articulated with dorsal part of socle. Connective anterior arms somewhat divergent, Y-shaped; stem present, $2 \mathrm{x}$ length of anterior arms; articulated with aedeagus. Segment X not strongly sclerotized.

Female genitalia. Pygofer with numerous macrosetae. Ovipositor not protruding far beyond pygofer apex. First valvula convex; dorsal sculpturing pattern strigate to reticulate; sculpturing submarginal; without distinctly delimited ventroapical sculpturing. Second valvula broad; gradually tapered; without dorsal median tooth; teeth on apical $1 / 3$ or more; teeth small, regularly or irregularly shaped. 


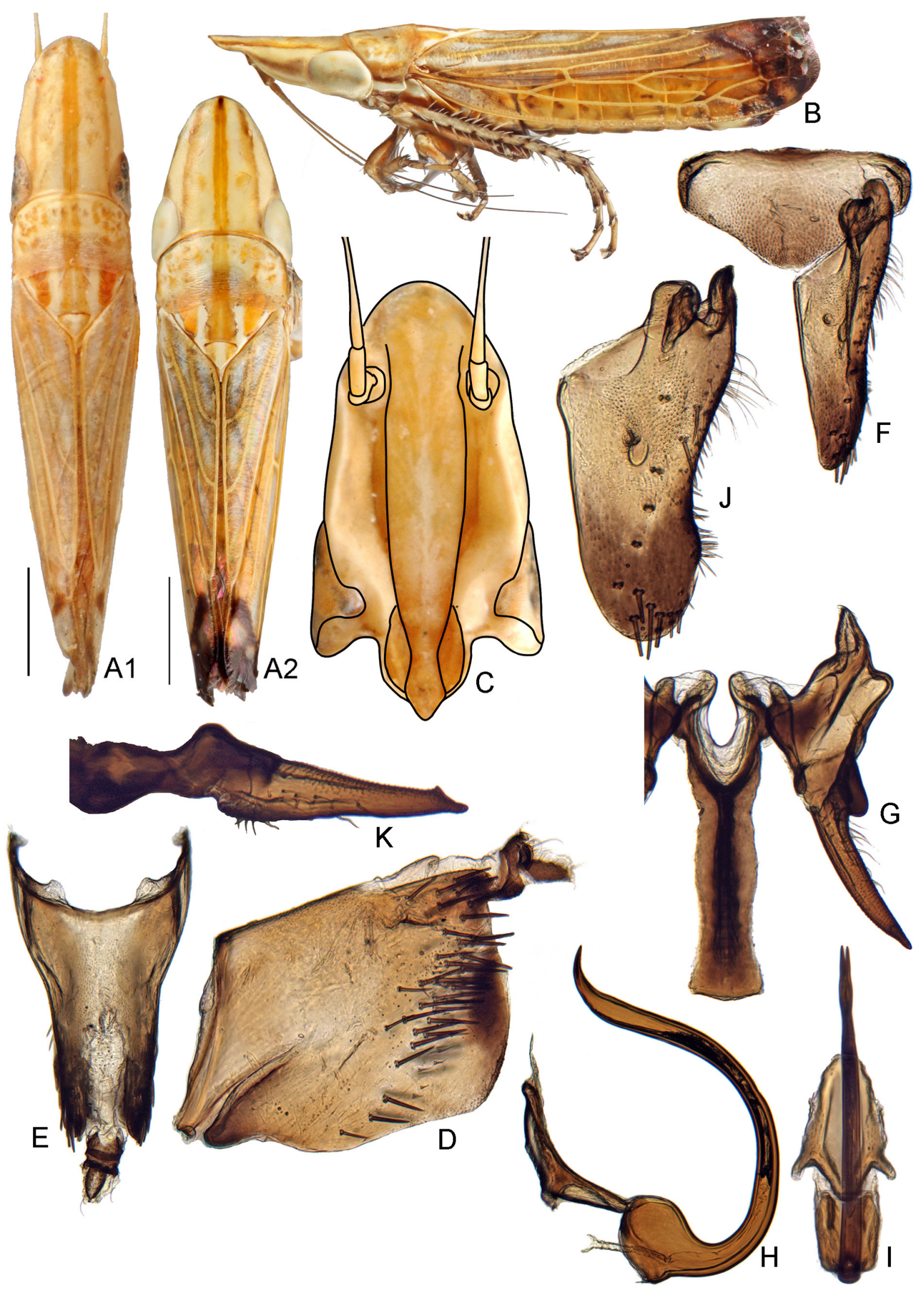

Fig. 37. Occinirvanini. Occinirvana eborea Evans, 1941. A-I. Standard views (see Material \& Methods). A1. $q$ (BMNH). A2, B. $\widehat{\jmath}$. J. Broad view of subgenital plate. K. Lateral view of apical half of style. 


\section{Geography and ecology}

Distribution: Western Australia. The only known species, Occinirvana eborea Evans, 1941, has been collected on Casuarina.

\section{Remarks}

Occinirvanini contains only the type genus with one species. The male genitalia of Occinirvana are illustrated and described here for the first time, and the phylogenetic analyses here are the first to include molecular data for the tribe. The male genitalia are for the most part typical for Deltocephalinae. The pattern of setae on the subgenital plate and the dorsal connective are somewhat unique. The characters of the male do not obviously suggest a relationship with other deltocephaline tribes or genera, but the molecular data very strongly supported a relationship with Loralia (Athysanini), another Australianendemic genus. The clade is placed in slightly different positions among the different analyses but always basal to the large derived deltocephaline clade shown in Fig. 3b. Despite this intriguing result, no unique morphological synapomorphies could be identified that would unite these two genera in a common tribe. Further study of these and other unique Australian taxa (e.g., Euleimonios Kirkaldy, 1906, Gunawardenea Fletcher \& Moir, 2008 and Occiplanocephalus Evans, 1941) should be conducted to explore their relationships to each other and to other Deltocephalinae, particularly since these three genera have some unique features of the male genitalia in common with Loralia (but not with Occinirvana). For now, Occinirvanini is retained as a monotypic tribe pending further study.

\section{Selected references}

Evans (1966), Dietrich (2004).

\section{Included genera}

Occinirvana Evans, 1941

Opsiini Emeljanov, 1962

Figs 38-41

Type genus: Opsius Fieber, 1866.

\section{Diagnosis}

Opsiini are small to large, stramineous, yellow, green, or brown leafhoppers. They can be identified by the bifurcate aedeagus with two shafts and gonopores. Some Mukariini and Ascius (Scaphytopiini) have a similarly divided aedeagus but Opsiini lack the other characters that define those groups.

\section{Description}

HEAD. Head subequal to or wider than or distinctly narrower than pronotum. Discal portion of crown glabrous with radial or longitudinal striae. Anterior margin of head shagreen, glabrous or irregularly textured, with numerous transverse striations, or foliaceous (Chlidochrus Emeljanov, 1962). Frontoclypeus not tumid; texture shagreen or glabrous. Clypellus parallel-sided, tapering apically, or widening apically; apex following or slightly surpassing normal curve of gena. Lorum subequal to or wider than clypellus near base. Antennal bases near middle or posteroventral (lower) corners of eyes. Antennae short, less than $1.5 \mathrm{x}$ width of head. Gena obtusely incised laterally; with fine erect seta beside laterofrontal suture. Antennal ledges absent or weakly developed (carinate or weakly carinate). Ocelli absent or reduced or present; close to eyes; on anterior margin of head.

Thorax. Pronotum lateral margin not carinate or carinate; lateral margin shorter than basal width of eye. 
WINGS. Forewing brachypterous to macropterous; appendix restricted to anal margin; with 3 anteapical cells; veins not raised; with or without reflexed costal veins; A1-A2 crossvein absent or present; apical venation not highly reticulate.

Legs. Profemur with AM1 seta only; intercalary row with one row of five or more fine setae; row AV with short, stout setae. Protibia dorsal surface rounded, convex. Metafemur apex macrosetae $2+0,2+1$, $2+2$, or $2+2+1$. Metatarsomere I not expanded apically; plantar setae simple, tapered.

Male genitalia. Apodemes of male sternites I and II often very well-developed. Valve articulated with pygofer; lateral margin short, articulating with pygofer at a point. Pygofer dorsoapical margin not strongly incised or incised to near mid-length; basolateral membranous cleft present; macrosetae absent or reduced ( $\leq$ two rows) or well differentiated into several rows. Subgenital plates free from each other; articulated with valve; without macrosetae or with macrosetae irregularly arranged or uniseriate laterally. Style broadly bilobed basally, median anterior lobe pronounced. Basal processes of the aedeagus/connective absent, reduced, or present and fused to base of aedeagus. Aedeagus shaft divided near base, with two gonopores, or shaft divided toward apex and forming semicircles (Circuliferina), with two gonopores. Connective anterior arms somewhat divergent, $\mathbf{Y}$ - or $\mathbf{U}$-shaped; articulated with aedeagus.

Female Genitalia. Pygofer with macrosetae reduced or absent or with numerous macrosetae. Ovipositor protruding or not protruding far beyond pygofer apex. First valvula convex or not strongly convex; dorsal sculpturing pattern strigate, concatenate, reticulate, with rectangular shaped cells, granulose, maculose, or imbricate (with overlapping scales); sculpturing reaching dorsal margin or submarginal; without distinctly delimited ventroapical sculpturing. Second valvula broad and gradually tapered, gradually broadened medially or subapically, or slender throughout; without dorsal median tooth; teeth on apical $1 / 3$ or more or restricted to apical $1 / 4$ or less; teeth small, regularly or irregularly shaped.

\section{Geography and ecology}

Distribution: cosmopolitan. Opsiini feed on a wide variety of herbaceous and woody dicots. Opsius stactogalus Fieber, 1866, a Palearctic species introduced in the New World, feeds on tamarix. This tribe contains several species of economic importance. Neoaliturus (Circulifer) tenellus (Baker, 1896) is the vector of beet curly top, tomato big bud, and 16SrV-16SrIX. N. (C.) haematoceps (Mulsant \& Rey, 1855) vectors sesame phyllody and 16SrV-16SrIX. Orosius orientalis (Matsumura, 1914) transmits tomato big bud, tobacco yellow dwarf, lucerne witches' broom, legume little leaf, mosaic I, potato purple top wilt, and witches' broom of groundnuts. Orosius albicinctus Distant, 1918 transmits sesame phyllody. Hishimonus phycitis (Distant, 1908) vectors eggplant little leaf. Hishimonoides sellatiformis Ishihara, 1965 and Hishimonus sellatus (Uhler, 1896) are vectors of mulberry dwarf and the latter leafhopper is also a vector of Rhus yellows, jujube witches' broom, and Cryptotaenia japonica witches' broom. Hishimonoides chinensis Anufriev, 1970 is a vector of jujube witches' broom.

\section{Remarks}

Opsiini contains 36 genera and 303 species. The phylogenetic analyses here included eight representatives of the tribe including the following that are included in analyses for the first time: Pseudophlepsius Zachvatkin, 1924, Orosius Distant, 1918, Nesophrosyne Kirkaldy, 1907, Japananus Ball, 1931 (previously in Scaphytopiini), and an undescribed genus near Libengaia from Zambia. The ML and Bayesian analyses resolved it as monophyletic but with low branch support, and the parsimony analysis resolved it as two separate monophyletic groups. The present phylogenetic analyses included more representatives of Opsiini than previous analyses and are the first to suggest the tribe is monophyletic. Strong branch support was recovered for some internal relationships within the tribe, but more representatives and more branch support are needed in future analyses to test the current subtribal classification. 
Opsiini is a morphologically diverse tribe. Except for the bifurcate aedeagus with two gonopores, there seem to be very few, if any, characters that can define the tribe entirely. It appears that the bifurcate aedeagus has arisen at least three times in the evolutionary history of Deltocephalinae. The finding that Japananus (previously included in Scaphytopiini based on the produced head and very broad gena) is related to Opsiini rather than Scaphytopiini underscores the utility of the bifurcate aedeagus as a predictor of relationships and helpful for classification at the tribal level. However, it is necessary to corroborate any taxonomic hypothesis based on this character with other morphological characters or molecular data. For example, Ascius DeLong, 1943 also has a bifurcate aedeagus, but it is retained in Scaphytopiini because it shares the numerous distinct reflexed costal veins and the widely separated anterior arms of the connective with Scaphytopius Ball, 1931, along with the produced head and broad gena.

Several genera (Afrascius Linnavuori, 1969; Japananus; Kirkaldiella Osborn, 1935; Masiripius Dlabola, 1981; Navaia Linnavuori, 1960; Phlepsopsius Dlabola, 1979 and Pugla Distant, 1908) are transferred to Opsiina from other tribes here because they share the bifurcate aedeagus and other similarities (e.g., brown irrorate color pattern) to some opsiines. Their placement to subtribe should be tested in future studies. Dixianus Ball, 1918 and Lycioides Oman, 1949 are included in Circuliferina following the suggestion of Emeljanov (1962) that Lycioides is closely related to Neoaliturus Distant, 1918, based in part on the shape of the aedeagus.

\section{Selected references}

Zimmerman (1948), Emeljanov (1962), Ghauri (1966), Knight (1970a, b), Linnavuori (1969), Dmitriev (2002), Dai et al. (2010), Dai et al. (2011), Bennett \& O'Grady (2011).

Included subtribes:

Achaeticina Emeljanov, 1962

Fig. 38

Type genus: Achaetica Emeljanov, 1959.

\section{Diagnosis}

Achaeticina are small, squat, brachypterous leafhoppers, stramineous, greenish, or brownish in color. They are distinguished from the other subtribes of Opsiini by the following combination of characters: forewings brachypterous and truncate, male pygofer lobes without ventral pointed processes, valve thick and strongly developed, valve wider than long, subgenital plates without macrosetae, aedeagal shafts arising from base, and ovipositor protruding far beyond pygofer apex.

\section{Description}

HeAD. Head subequal to or wider than pronotum or distinctly narrower than pronotum. Discal portion of crown glabrous with radial or longitudinal striae or shagreen. Anterior margin of head shagreen, glabrous, irregularly textured, or foliaceous. Frontoclypeus not tumid; texture shagreen or glabrous. Clypellus parallel-sided, tapering apically, or widening apically; apex following or slightly surpassing normal curve of gena. Lorum subequal to or wider than clypellus near base. Antennal bases near middle or posteroventral (lower) corners of eyes. Antennae short, less than $1.5 \mathrm{x}$ width of head. Gena obtusely incised laterally; with fine erect seta beside laterofrontal suture. Antennal ledges absent or weakly developed (carinate or weakly carinate). Ocelli absent or reduced in size; on anterior margin of head if present.

THORAX. Pronotum lateral margin not carinate; lateral margin shorter than basal width of eye. 


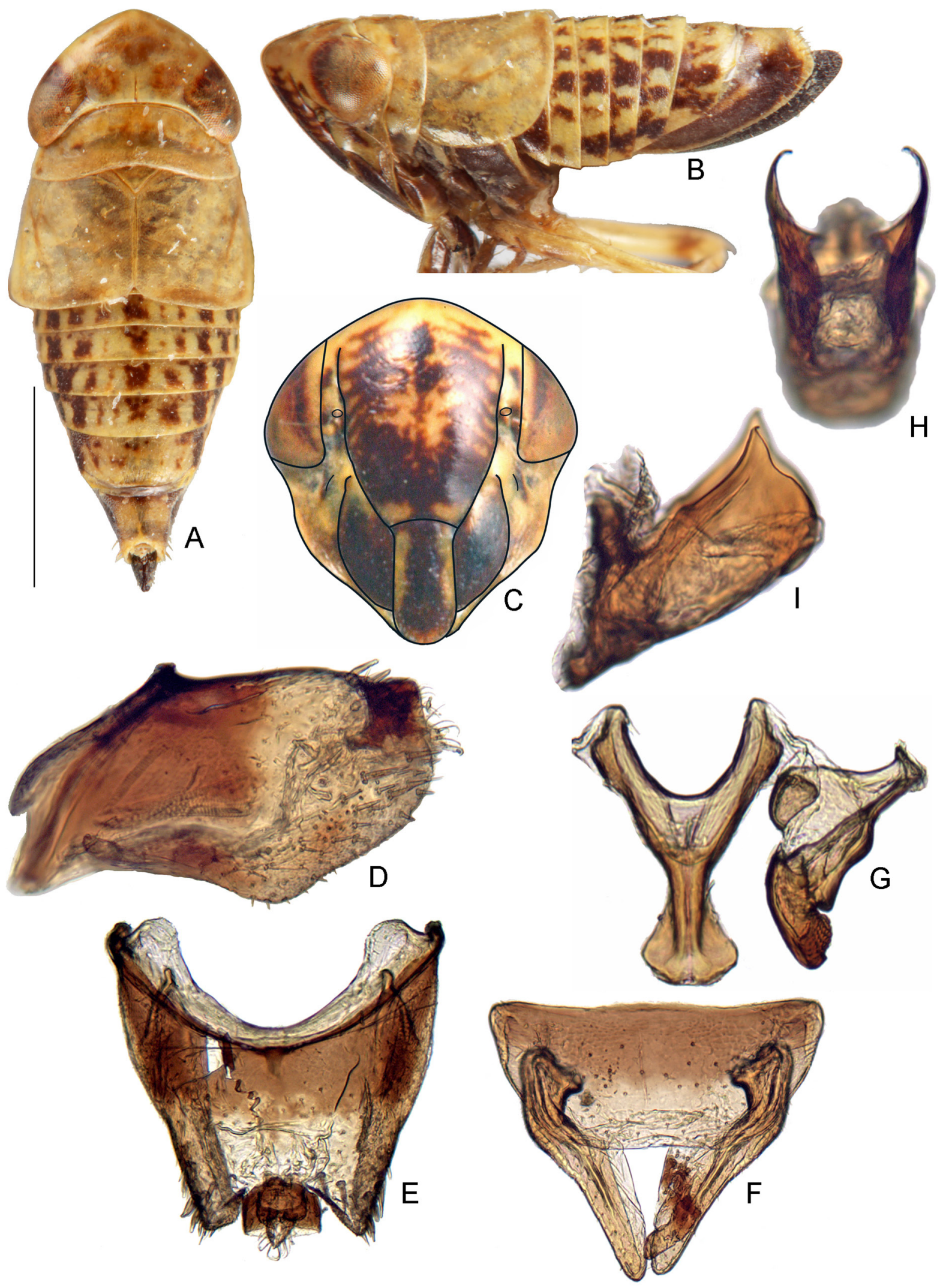

Fig. 38. Opsiini (Achaeticina). Achaetica anabasidis Emeljanov, 1959. A-I. Standard views (see Material \& Methods). 
WINGS. Forewing brachypterous or subbrachypterous; veins not raised.

LEGs. Profemur with AM1 seta only; intercalary row with one row of five or more fine setae; row AV with short, stout setae. Protibia dorsal surface rounded, convex. Metafemur apex macrosetae $2+0,2+1$, $2+2$, or $2+2+1$. Metatarsomere I not expanded apically; plantar setae simple, tapered.

MaLe genitalia. Valve articulated with pygofer; with short point of articulation with pygofer. Pygofer dorsoapical margin not strongly incised; basolateral membranous cleft present; macrosetae absent or reduced ( $\leq$ two rows). Subgenital plates free from each other, articulated with valve; without macrosetae. Style broadly bilobed basally, median anterior lobe pronounced. Basal processes of the aedeagus/connective absent. Aedeagus without basal hinge; shaft divided near base, with two gonopores. Connective anterior arms somewhat divergent, $\mathbf{Y}$-shaped; articulated with aedeagus.

Female genitalia. Pygofer with macrosetae reduced or absent. Ovipositor protruding far beyond pygofer apex. First valvula convex, dorsal sculpturing pattern granulose, maculose, or imbricate (with overlapping scales); sculpturing submarginal; without distinctly delimited ventroapical sculpturing. Second valvula gradually broadened medially or subapically; without dorsal median tooth; teeth restricted to apical $1 / 4$ or less; teeth small, regularly or irregularly shaped.

\section{Geography and ecology}

Distribution: Palearctic (Central Asia).

\section{Remarks}

Achaeticina contains 4 genera and 32 species.

\section{Included genera}

Achaetica Emeljanov, 1959

Chlidochrus Emeljanov, 1962

Diacra Emeljanov, 1961

Zapycna Emeljanov, 1968

Circuliferina Emeljanov, 1962

Fig. 39

Type genus: Circulifer Zachvatkin, 1935.

\section{Diagnosis}

Circuliferina are relatively small and slender ivory, greenish, or brownish leafhoppers. They are distinguished from other subtribes of Opsiini by their relatively small, slender shape, mesal margin of eye notched, forewing macropterous, subgenital plate with a lateral row of macrosetae, valve normal, aedeagus bifurcate apically with branches forming semicircles, and ovipositor not protruding far beyond pygofer apex.

\section{Description}

HEAD. Head subequal to or wider than pronotum. Discal portion of crown glabrous with radial or longitudinal striae. Anterior margin of head shagreen. Frontoclypeus not tumid; texture shagreen. Clypellus parallel-sided or tapering apically; apex following or slightly surpassing normal curve of gena. Lorum subequal to or wider than clypellus near base. Antennal bases near middle or posteroventral (lower) corners of eyes. Antennae short, less than 1.5 x width of head. Gena obtusely incised laterally; 


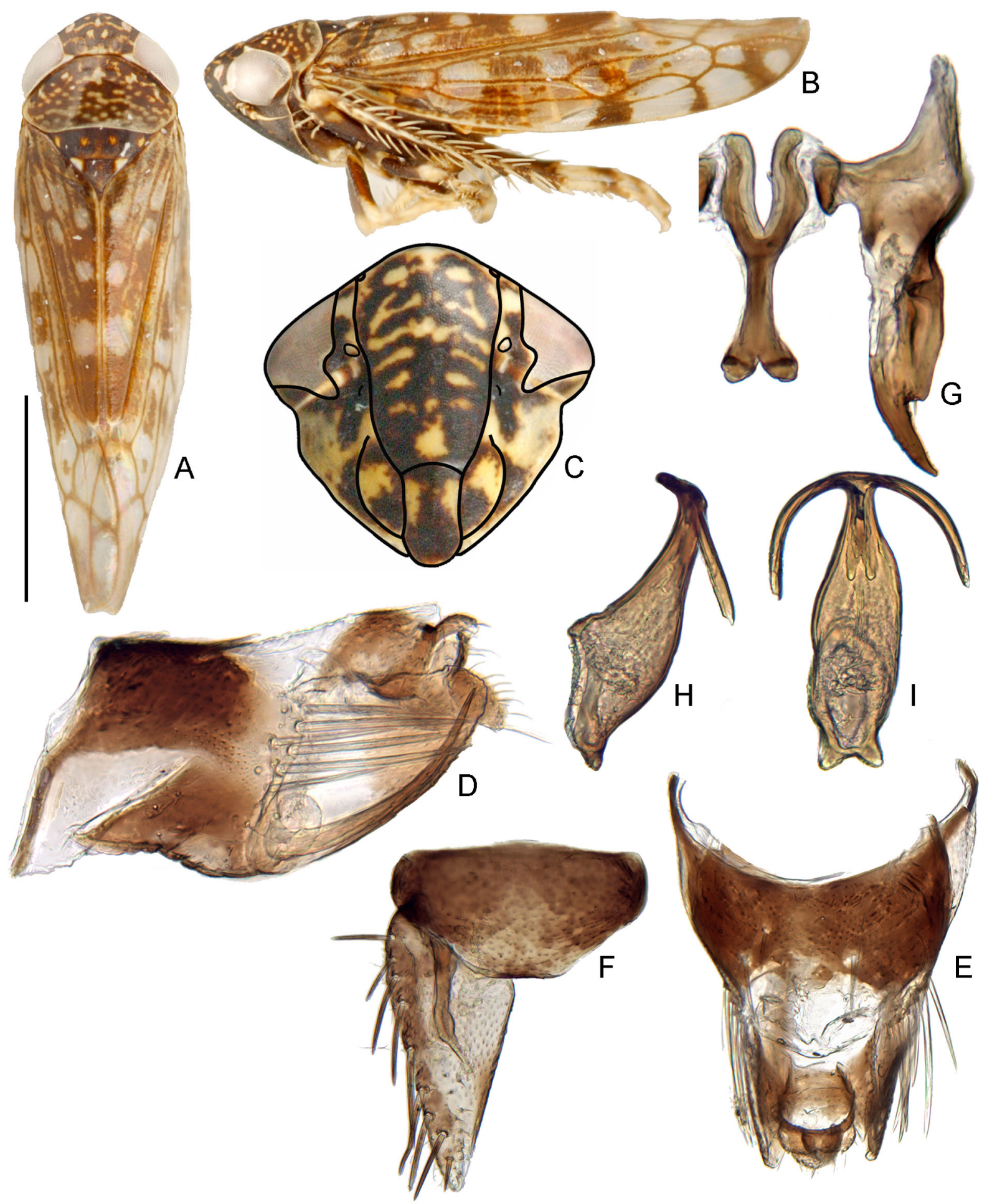

Fig. 39. Opsiini (Circuliferina). Neoaliturus carbonarius Mitjaev, 1971. A-I. Standard views (see Material \& Methods). 
with fine erect seta beside laterofrontal suture. Antennal ledges absent. Ocelli present; close to eyes; on anterior margin of head. Mesal margin of eye notched.

THORAX. Pronotum lateral margin carinate; lateral margin shorter than basal width of eye.

Wings. Forewing macropterous; appendix restricted to anal margin; with 3 anteapical cells; veins not raised; with or without reflexed costal veins; A1-A2 crossvein absent; apical venation not highly reticulate.

Legs. Profemur with AM1 seta only; intercalary row with one row of five or more fine setae; row AV with short, stout setae. Protibia dorsal surface rounded, convex. Metafemur apex macrosetae $2+2+1$. Metatarsomere I not expanded apically; plantar setae simple, tapered.

Male Genitalia. Valve articulated with pygofer; with short point of articulation with pygofer. Pygofer basolateral membranous cleft present; macrosetae well differentiated into several rows. Subgenital plates free from each other, articulated with valve; macrosetae uniseriate laterally. Style broadly bilobed basally, median anterior lobe pronounced. Basal processes of the aedeagus/connective absent. Aedeagus without basal hinge; shaft divided toward apex, with two gonopores. Connective anterior arms somewhat divergent, Y-shaped; articulated with aedeagus.

Female genitalia. Pygofer with numerous macrosetae. Ovipositor not protruding far beyond pygofer apex. First valvula convex; dorsal sculpturing pattern granulose, maculose, or imbricate (with overlapping scales); sculpturing reaching dorsal margin; without distinctly delimited ventroapical sculpturing. Second valvula slender throughout; without dorsal median tooth; teeth on apical $1 / 3$ or more; teeth small, regularly or irregularly shaped.

\section{Geography and ecology}

Distribution: cosmopolitan, Neoaliturus (Circulifer) tenellus (Baker, 1896) is adventive in the New World.

\section{Remarks}

Circuliferina contains 5 genera and 45 species.

\section{Included genera}

Concavifer Dlabola, 1960

Dixianus Ball, 1918 placement nov. (transferred from Athysanini)

Lycioides Oman, 1949 placement nov. (transferred from Athysanini)

Neoaliturus Distant, 1918

Pedarium Emeljanov, 1961

Eremophlepsiina Dmitriev, 2006

Fig. 40

Type genus: Eremophlepsius Zachvatkin, 1924.

\section{Diagnosis}

Eremophlepsiina are medium sized, robust, ivory or brownish leafhoppers. They are distinguished from other subtribes of Opsiini by the macropterous forewings, pygofer lobes with ventral pointed processes, valve thick and strongly developed, valve longer than wide and parabolically or lanceolate-parabolically shaped, subgenital plates without macrosetae, and aedeagal shafts arising from base. 


\section{Description}

HEAD. Head subequal to, wider than, or distinctly narrower than pronotum. Discal portion of crown glabrous with radial or longitudinal striae. Anterior margin of head glabrous, irregularly textured, with numerous transverse striations. Frontoclypeus not tumid; texture shagreen. Clypellus widening apically; apex following or slightly surpassing normal curve of gena. Lorum subequal to or wider than clypellus near base. Antennal bases near middle or posteroventral (lower) corners of eyes. Antennae short, less than $1.5 \mathrm{x}$ width of head. Gena obtusely incised laterally; with fine erect seta beside laterofrontal suture. Antennal ledges absent. Ocelli present; close to eyes; on anterior margin of head.

Thorax. Pronotum lateral margin carinate; lateral margin shorter than basal width of eye.

WINGS. Forewing macropterous; appendix restricted to anal margin; with 3 anteapical cells; veins not raised; with or without reflexed costal veins; A1-A2 crossvein present; apical venation not highly reticulate.

Legs. Profemur with AM1 seta only; intercalary row with one row of five or more fine setae; row $\mathrm{AV}$ with short, stout macrosetae. Protibia dorsal surface rounded, convex. Metafemur apex macrosetae $2+2+1$. Metatarsomere I not expanded apically; plantar setae simple, tapered.

MaLE Genitalia. Valve articulated with pygofer; with short point of articulation with pygofer; thick and well-developed; longer than wide, parabolically or lanceolate-parabolically shaped. Pygofer dorsoapical margin incised to near mid-length; basolateral membranous cleft present; macrosetae well differentiated into several rows. Subgenital plates free from each other; articulated with valve; with thin hair-like setae or with macrosetae scattered, irregularly arranged. Style broadly bilobed basally, median anterior lobe pronounced. Basal processes of the aedeagus present, fused near base of aedeagus. Aedeagus without basal hinge; shaft divided near base, with two gonopores. Connective anterior arms somewhat divergent, Y-shaped; articulated with aedeagus.

Female genitalia. Pygofer with numerous macrosetae. Ovipositor protruding far beyond pygofer apex. First valvula not strongly convex; dorsal sculpturing pattern strigate, concatenate, reticulate, or with rectangular shaped cells; sculpturing reaching dorsal margin; without distinctly delimited ventroapical sculpturing. Second valvula broad, gradually tapered; without dorsal median tooth; teeth on apical 1/3 or more; teeth small, regularly or irregularly shaped.

\section{Geography and ecology}

Distribution: Palearctic.

\section{Remarks}

Eremophlepsiina contains 2 genera and 5 species. It is closely related to Achaeticina.

\section{Included genera}

Eremophlepsius Zachvatkin, 1924

Pseudophlepsius Zachvatkin, 1924 


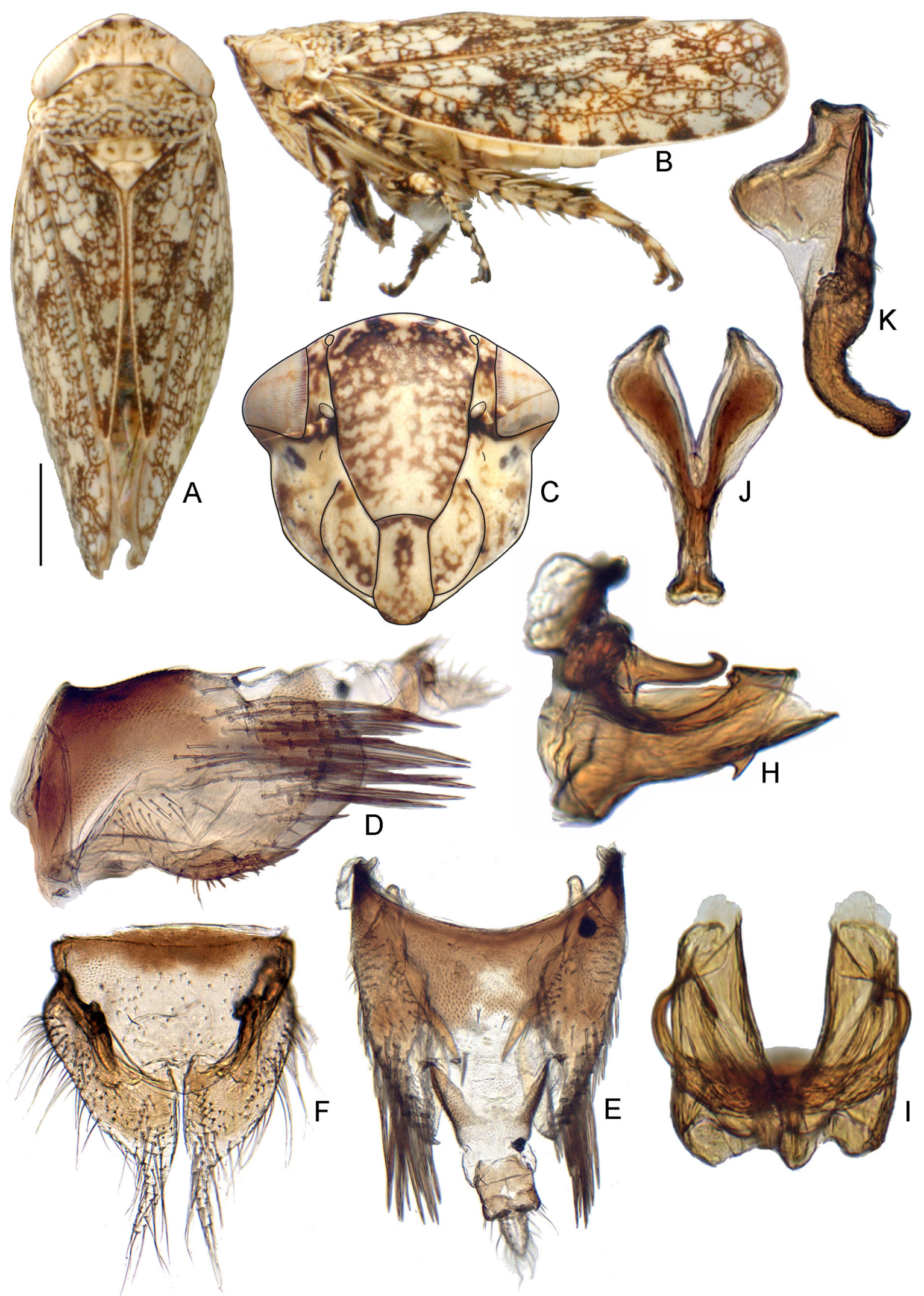

Fig. 40. Opsiini (Eremophlepsiina). Pseudophlepsius binotatus (Signoret, 1880). A-F, H-I. Standard views (see Material \& Methods). I. Caudoventral view. J. Connective. K. Style. 
Opsiina Emeljanov, 1962

Fig. 41

Type genus: Opsius Fieber, 1866.

\section{Diagnosis}

Opsiina are medium sized to large, robust leafhoppers, variable in color but often yellowish, greenish, or brownish and with reticulate or irrorate brown markings; brown markings sometimes saddle-shaped. They are distinguished from other subtribes of Opsiini by the macropterous forewings, subgenital plates with a lateral row of macrosetae, subgenital plate apex often digitate and membranous, valve parabolically shaped, aedeagal shafts arising from base, and ovipositor not protruding far beyond pygofer apex.

\section{Description}

HEAD. Head subequal to or wider than pronotum. Discal portion of crown glabrous with radial or longitudinal striae. Anterior margin of head shagreen. Frontoclypeus not tumid; texture shagreen. Clypellus widening apically; apex following or slightly surpassing normal curve of gena. Lorum subequal to or wider than clypellus near base. Antennal bases near middle or posteroventral (lower) corners of eyes. Antennae short, less than $1.5 \mathrm{x}$ width of head. Gena obtusely incised laterally; with fine erect seta beside laterofrontal suture. Antennal ledges absent. Ocelli present; close to eyes; on anterior margin of head.

THORAX. Pronotum lateral margin carinate; lateral margin shorter than basal width of eye.

WINGS. Forewing macropterous; appendix restricted to anal margin; with 3 anteapical cells; veins not raised; without or with reflexed costal veins; A1-A2 crossvein absent or present; apical venation not highly reticulate.

Legs. Profemur with AM1 seta only; intercalary row with one row of five or more fine setae; row AV with short, stout setae. Protibia dorsal surface rounded, convex. Metafemur apex macrosetae $2+2+1$. Metatarsomere I not expanded apically; plantar setae simple, tapered.

MaLe Genitalia. Valve articulated with pygofer; with short point of articulation with pygofer. Pygofer basolateral membranous cleft present; macrosetae well differentiated into several rows. Subgenital plates free from each other, articulated with valve; macrosetae uniseriate laterally. Style broadly bilobed basally, median anterior lobe pronounced. Basal processes of the aedeagus/connective absent or reduced or present, fused to base of aedeagus. Aedeagus without basal hinge; shaft divided near base, with two gonopores. Connective anterior arms somewhat divergent, $\mathbf{Y}$ - or $\mathbf{U}$-shaped; articulated with aedeagus.

Female Genitalia. Pygofer with numerous macrosetae. Ovipositor not protruding far beyond pygofer apex. First valvula convex; dorsal sculpturing pattern concatenate, reticulate, or with rectangular shaped cells; sculpturing reaching dorsal margin; without or with indistinctly delimited ventroapical sculpturing. Second valvula broad, gradually tapered or slender throughout; without dorsal median tooth; teeth on apical $1 / 3$ or more; teeth small, regularly or irregularly shaped.

\section{Geography and ecology}

Distribution: cosmopolitan. Opsius stactogalus Fieber, 1866 is adventive in the New World and feeds on Tamarix.

\section{Remarks}

Opsiina is the largest of the 4 subtribes. It contains 25 genera and 218 species. 


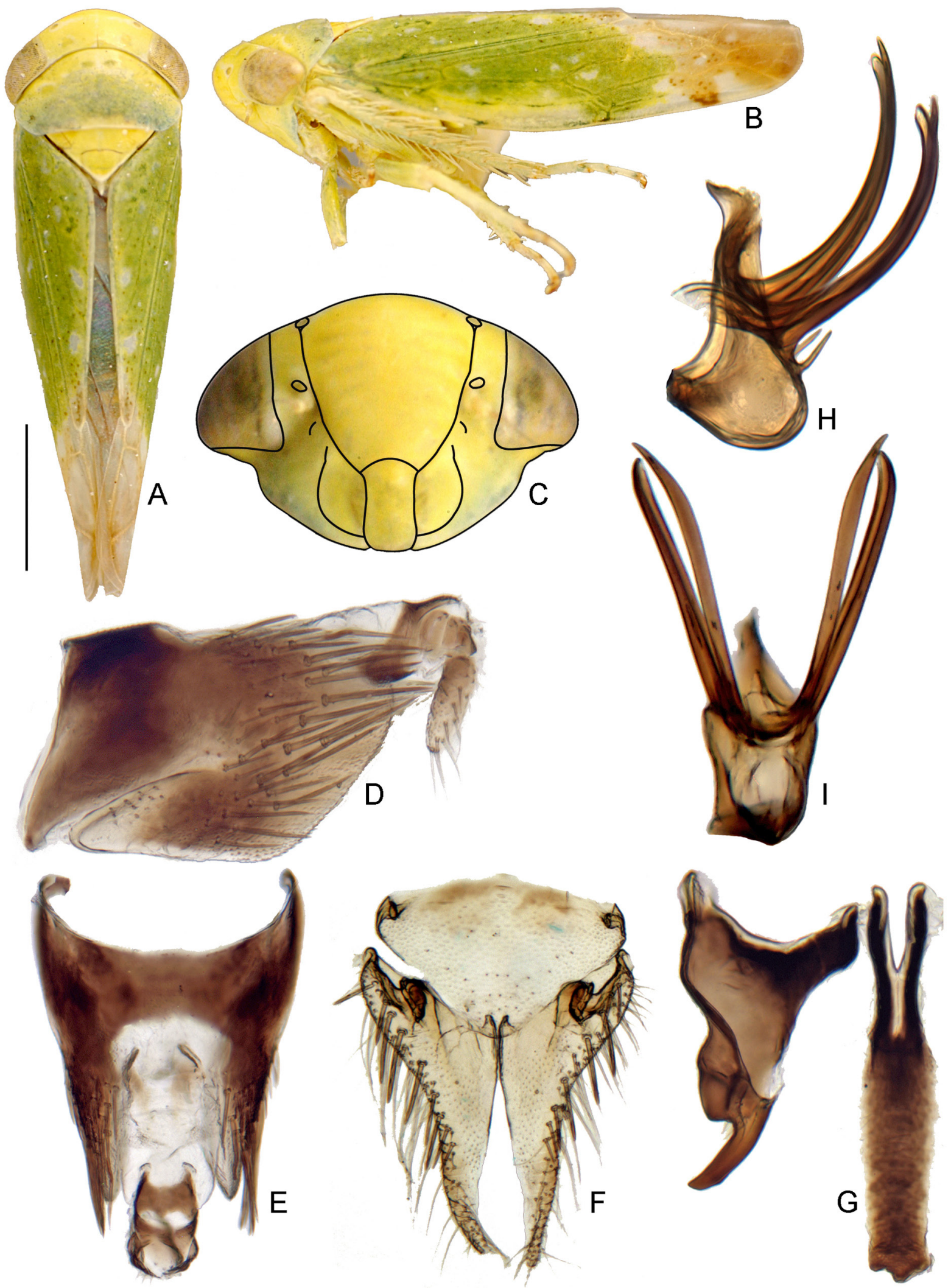

Fig. 41. Opsiini (Opsiina). Opsius stactogalus Fieber, 1866. A-I. Standard views (see Material \& Methods). 


\section{Included genera}

Afrascius Linnavuori, 1969 placement nov. (transferred from Scaphytopiini)

Aladzoa Linnavuori, 1969

Alishania Vilbaste, 1969

Hishimonoides Ishihara, 1965

Hishimonus Ishihara, 1953

Introrsa Dai \& Zhang, 2010

Japananus Ball, 1931 placement nov. (transferred from Scaphytopiini)

Kirkaldiella Osborn, 1935 placement nov. (transferred from Athysanini)

Lampridius Distant, 1918

Libengaia Linnavuori, 1969

Litura Knight, 1970

Masiripius Dlabola, 1981 placement nov. (transferred from Platymetopiina)

Naevus Knight, 1970

Navaia Linnavuori, 1960 placement nov. (previously unplaced in Deltocephalinae)

Nesophrosyne Kirkaldy, 1907

Norva Emeljanov, 1969

Opsianus Linnavuori, 1960

Opsius Fieber, 1866

Orosius Distant, 1918

Paralampridius Dai, Dietrich \& Zhang, 2011

Phlepsopsius Dlabola, 1979 placement nov. (transferred from Athysanini)

Pugla Distant, 1908 placement nov. (transferred from Platymetopiina)

Satsumanus Ishihara, 1953

Xerophytacolus Stiller, 2012

Xerophytavorus Stiller, 2012

Paralimnini Distant, 1908

Figs 42,43

Type genus: Paralimnus Matsumura, 1902.

\section{Diagnosis}

Paralimnini are small to medium sized leafhoppers. They can be identified by the combination of the following characters: clypellus tapering apically or parallel-sided, lorum narrower than clypellus at base; connective with anterior arms closely appressed, articulated with aedeagus; female first valvula sculpturing imbricate or rarely maculose or granulose. The tribe is very similar morphologically to the closely related Deltocephalini, from which it can be distinguished by the articulation between the connective and aedeagus (fused in Deltocephalini), although a few species of Flexamia (Paralimnini) have the connective fused to the aedeagus.

\section{Description}

HeAd. Head subequal to or wider than pronotum. Discal portion of crown glabrous with radial or longitudinal striae, or shagreen. Anterior margin of head shagreen. Frontoclypeus not tumid or tumid; texture shagreen. Clypellus parallel-sided or tapering apically; apex following or slightly surpassing normal curve of gena. Lorum distinctly narrower than clypellus near base. Antennal bases near middle or posteroventral (lower) corners of eyes. Antennae short, less than $1.5 \mathrm{x}$ width of head. Gena obtusely incised laterally; with fine erect seta beside laterofrontal suture. Antennal ledges absent. Ocelli present; close to eyes; on anterior margin of head. 
THorax. Pronotum lateral margin not carinate; lateral margin shorter than basal width of eye.

WINGS. Forewing macropterous to brachypterous; if macropterous then appendix absent, reduced, or present and restricted to anal margin; with 3 anteapical cells; veins not raised; without or rarely with reflexed costal veins; crossvein r-m1 often connected to R basad of its fork; A1-A2 crossvein absent; apical venation not highly reticulate.

LEGS. Profemur with AM1 seta only; intercalary row with one row of five or more fine setae; row AV usually with short, stout setae or rarely with relatively long macrosetae. Protibia dorsal surface rounded, convex. Metafemur apex macrosetae $2+2+1$. Metatarsomere I not expanded apically; plantar setae simple, tapered or rarely with one or more platellae.

MaLE Genitalia. Valve articulated with pygofer; lateral margin short, articulating with pygofer at a point. Pygofer dorsoapical margin incised to near mid-length; basolateral membranous cleft present; macrosetae well differentiated into several rows. Subgenital plates free from each other; usually articulated with valve, or rarely fused; macrosetae uniseriate laterally, with two lateral rows of macrosetae, with some irregularly arranged macrosetae near lateral margin, or reduced or absent. Style broadly bilobed basally, median anterior lobe pronounced. Basal processes of the aedeagus/connective absent or reduced or present, connected or articulated to connective or near base of aedeagus. Aedeagus with single shaft and gonopore. Connective anterior arms closely appressed anteriorly, linear- or loop-shaped); nearly always articulated with aedeagus, rarely fused to aedeagus (e.g., some Flexamia).

Female genitalia. Pygofer with numerous macrosetae. Ovipositor not protruding or rarely protruding far beyond pygofer apex. First valvula convex or not strongly convex; dorsal sculpturing pattern imbricate (with overlapping scales) or rarely granulose or maculose; sculpturing reaching dorsal margin or submarginal; without or rarely with distinctly delimited ventroapical sculpturing. Second valvula broad, gradually tapered; without dorsal median tooth; teeth on apical $1 / 3$ or more; teeth small, regularly or irregularly shaped or absent.

\section{Geography and ecology}

Distribution: cosmopolitan. Paralimnini feed on grasses or sedges and are often abundant in grassland ecosystems. A case study of the North American genus Flexamia (Whitcomb \& Hicks 1988; Dietrich et al. 1997) examined the roles of host plants, geography, phenology, and phylogeny in the species level diversification of the genus. These studies showed that related species groups feed on the same or similar species of grass hosts and that host shifts occurred in the past, sometimes to distantly related grass species. Geographic partitioning of the grass host ranges and phenological differences of the leafhopper species contributed to the speciation of the group.

Psammotettix striatus (Linnaeus, 1758) and P. alienus (Dahlbom, 1850) transmit wheat dwarf virus and mosaic virus of winter wheat.

\section{Remarks}

Paralimnini contains 139 genera and 931 species. It is closely related to Deltocephalini, with which it shares a similar head morphology and the linear connective. Tetartostylini, which has a similar connective articulated with the aedeagus but has the subgenital plates fused to the valve and with unique apical spines, is also closely related to Paralimnini. Many species in understudied areas of the world remain to be described. For example, in just three publications, Stiller $(2009 ; 2010 \mathrm{a}, \mathrm{b})$ described 59 new species in three genera of Paralimnini from South Africa. 


\section{Selected references}

Emeljanov (1962), Knight (1974), Whitcomb \& Hicks (1988), Webb \& Heller (1990), Dietrich et al. (1997), Kamitani (1999), Dmitriev (2004b), Stiller (2009, 2010a, b).

Included subtribes:

Aglenina Dmitriev, 2004

Fig. 42

Type genus: Aglena Amyot \& Serville, 1843.

\section{Diagnosis}

Aglenina can be distinguished from other Paralimnini by their large size and black and yellow coloration.

\section{Remarks}

Aglena ornata Herrich-Schäffer, 1838 is the only species belonging to this subtribe. It occurs in the Palearctic region.

\section{Included genera}

Aglena Amyot \& Serville, 1843.

Paralimnina Distant, 1908

Fig. 43

Type genus: Paralimnus Matsumara, 1902.

\section{Diagnosis}

Paralimnina can be distinguished from Aglenina by their smaller size and their coloration, which is typically not black and yellow as in Aglena ornata.

\section{Description}

As in tribal description.

\section{Remarks}

Paralimnina contains 138 genera and 930 species.

Only the genera occurring in European Russia and adjacent territories were considered in the placement of genera to subtribes recognized by Dmitriev (2004). All of the other genera that were left unplaced to subtribe in Paralimnini are placed here in Paralimnina. Commellus Osborn \& Ball, 1902 and Flexarida Whitcomb \& Hicks, 1993 were both listed as unplaced to tribe in Deltocephalinae by Oman et al. (1990) and are placed here in Paralimnina.

Pasaremus is transferred to Paralimnina from Cicadulini, in which it was placed by Oman et al. (1990). It does not possess the characteristic long sclerotized segment $\mathrm{X}$ or other features of Cicadulini, and it possesses a tapered clypellus and a 'linear' connective articulated with the aedeagus which are characteristic of Paralimnina.

\section{Included genera}

Acharis Emeljanov, 1966 placement nov. (previously unplaced in Paralimnini) 


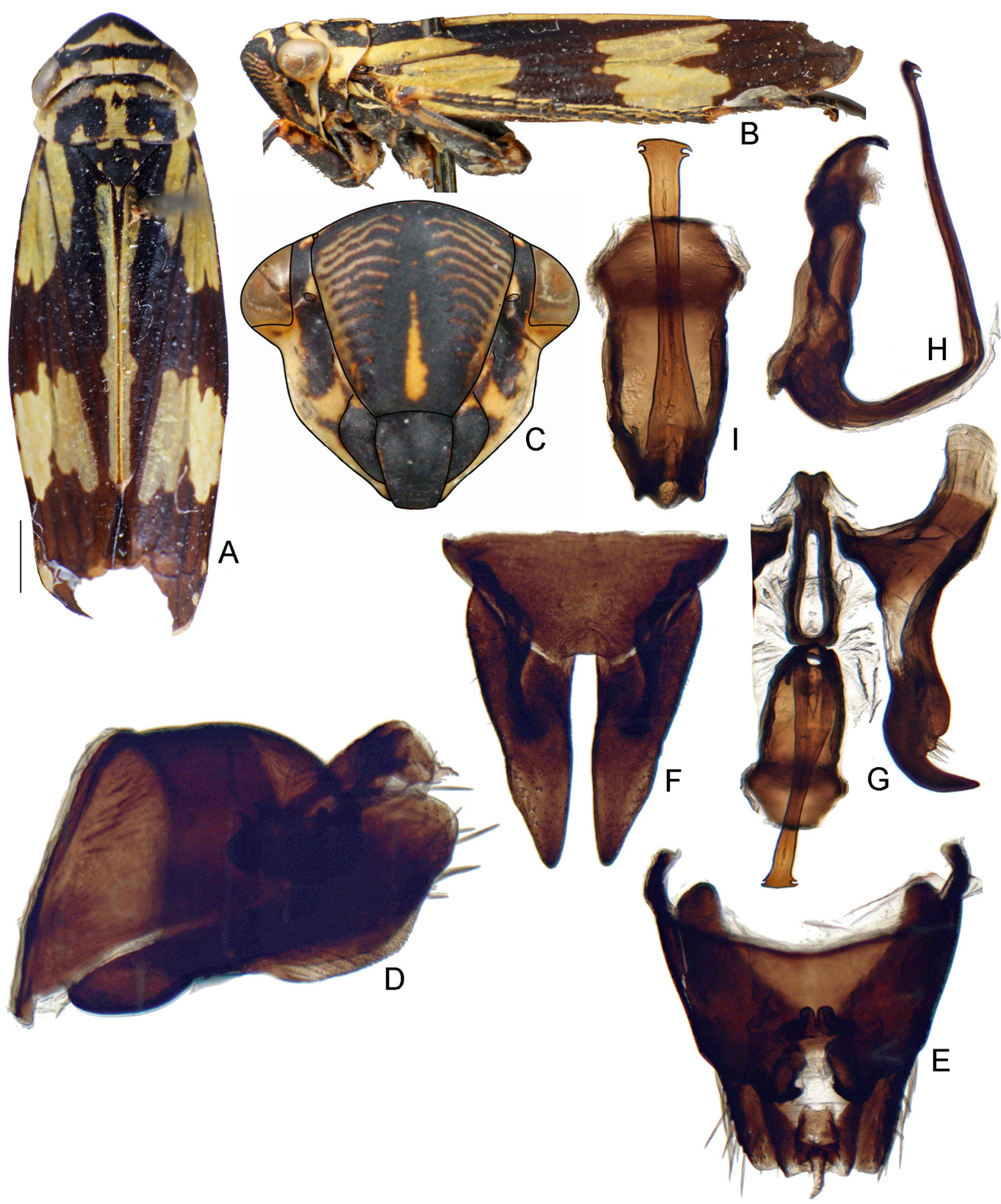

Fig. 42. Paralimnini (Aglenina). Aglena ornata (Herrich-Schäffer, 1838). A-I. Standard views (see Material \& Methods). G. Connective and style shown with aedeagus. 


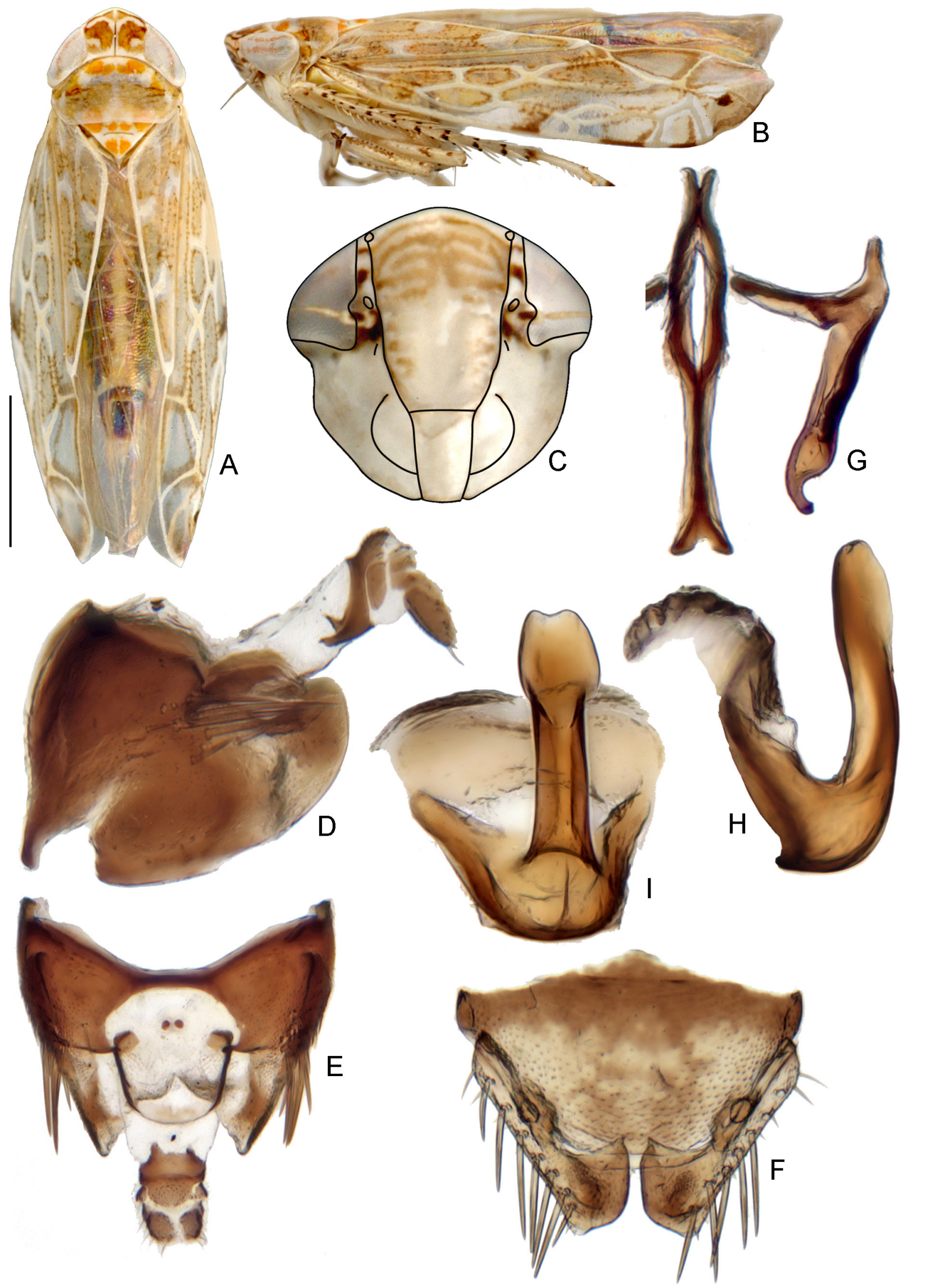

Fig. 43. Paralimnini (Paralimnina). A-C. Paralimnus angusticeps Zachvatkin, 1953. D-I. Psammotettix striatus (Linnaeus, 1758). A-I. Standard views (see Material \& Methods). 
Adarrus Ribaut, 1946 placement nov. (previously unplaced in Paralimnini) Aflexia Oman, 1949 placement nov. (previously unplaced in Paralimnini) Agudus Oman, 1938 placement nov. (previously unplaced in Paralimnini) Alapus DeLong \& Sleesman, 1929 placement nov. (previously unplaced in Paralimnini)

Altaiotettix Vilbaste, 1965

Anareia Vilbaste, 1965 placement nov. (previously unplaced in Paralimnini)

Anargella Emeljanov, 1972 placement nov. (previously unplaced in Paralimnini)

Araldus Ribaut, 1946 placement nov. (previously unplaced in Paralimnini)

Arocephalus Ribaut, 1946

Arthaldeus Ribaut, 1946

Auridius Oman, 1949 placement nov. (previously unplaced in Paralimnini)

Bhavapura Chalam \& Rama Subba Rao, 2005 placement nov. (previously unplaced in Paralimnini)

Calamotettix Emeljanov, 1959 placement nov. (previously unplaced in Paralimnini)

Caloduferna Webb, 1980 placement nov. (previously unplaced in Paralimnini)

Canariotettix Lindberg, 1954 placement nov. (previously unplaced in Paralimnini)

Caphodellus Linnavuori \& DeLong, 1976 placement nov. (previously unplaced in Paralimnini)

Cazenus Oman, 1949 placement nov. (previously unplaced in Paralimnini)

Cedarotettix Theron, 1975 placement nov. (previously unplaced in Paralimnini)

Changwhania Kwon, 1980 placement nov. (previously unplaced in Paralimnini)

Chelidinus Emeljanov, 1962 placement nov. (previously unplaced in Paralimnini)

Chloothea Emeljanov, 1959

Cleptochiton Emeljanov, 1959 placement nov. (previously unplaced in Paralimnini)

Coelestinus Emeljanov, 1962

Coganus Theron, 1978 placement nov. (previously unplaced in Paralimnini)

Commellus Osborn \& Ball, 1902 placement nov. (previously unplaced in Deltocephalinae)

Connectivus Xing \& Li, 2012 placement nov. (previously unplaced in Paralimnini)

Cosmotettix Ribaut, 1942 placement nov. (previously unplaced in Paralimnini)

Cribrus Oman, 1949 placement nov. (previously unplaced in Paralimnini)

Ctenotettix Novikov \& Anufriev, 2005 placement nov. (previously unplaced in Paralimnini)

Cumbrenanus DeLong \& Cwikla, 1984 placement nov. (previously unplaced in Paralimnini)

Destitutus Xing \& Li, 2011 placement nov. (previously unplaced in Paralimnini)

Diplocolenus Ribaut, 1946 placement nov. (previously unplaced in Paralimnini)

Ebarrius Ribaut, 1946

Elginus Theron, 1975 placement nov. (previously unplaced in Paralimnini)

Emeljanovianus Dlabola, 1965

Enantiocephalus Haupt, 1926

Errastunus Ribaut, 1946

Erzaleus Ribaut, 1952 placement nov. (previously unplaced in Paralimnini)

Falcitettix Linnavuori, 1953 placement nov. (previously unplaced in Paralimnini)

Flexamia DeLong, 1926 placement nov. (previously unplaced in Paralimnini)

Flexarida Whitcomb \& Hicks, 1993 placement nov. (previously unplaced in Deltocephalinae)

Futasujinoidella Kwon \& Lee, 1979 placement nov. (previously unplaced in Paralimnini)

Futasujinus Ishihara, 1953 placement nov. (previously unplaced in Paralimnini)

Giprus Oman, 1949 placement nov. (previously unplaced in Paralimnini)

Gobicuellus Dlabola, 1967 placement nov. (previously unplaced in Paralimnini)

Goldeus Ribaut, 1946 placement nov. (previously unplaced in Paralimnini)

Hebecephalus DeLong, 1926 placement nov. (previously unplaced in Paralimnini)

Hebexa Oman, 1949 placement nov. (previously unplaced in Paralimnini)

Hengchunia Vilbaste, 1969 placement nov. (previously unplaced in Paralimnini)

Henschia Lethierry, 1892 
Hiltus Theron, 1974 placement nov. (previously unplaced in Paralimnini)

Histipagus Remane \& Asche, 1980 placement nov. (previously unplaced in Paralimnini)

Ibadarrus Remane \& Asche, 1980 placement nov. (previously unplaced in Paralimnini)

Jannius Theron, 1982 placement nov. (previously unplaced in Paralimnini)

Jassargus Zachvatkin, 1933

Jilinga Ghauri, 1974 placement nov. (previously unplaced in Paralimnini)

Kaszabinus Dlabola, 1965 placement nov. (previously unplaced in Paralimnini)

Kazachstanicus Dlabola, 1961 placement nov. (previously unplaced in Paralimnini)

Ladya Theron, 1982 placement nov. (previously unplaced in Paralimnini)

Laevicephalus DeLong, 1926 placement nov. (previously unplaced in Paralimnini)

Latalus DeLong \& Sleesman, 1929

Lebradea Remane, 1959 placement nov. (previously unplaced in Paralimnini)

Lecacis Theron, 1982 placement nov. (previously unplaced in Paralimnini)

Lemellus Oman, 1949 placement nov. (previously unplaced in Paralimnini)

Maximianus Distant, 1918 placement nov. (transferred from Athysanini)

Mayawa Fletcher, 2000 placement nov. (previously unplaced in Paralimnini)

Mendrausus Ribaut, 1946 placement nov. (previously unplaced in Paralimnini)

Mendreus Ribaut, 1946 placement nov. (previously unplaced in Paralimnini)

Mesolimnella Dlabola, 1994 placement nov. (previously unplaced in Paralimnini)

Metagoldeus Remane \& Asche, 1980 placement nov. (previously unplaced in Paralimnini)

Metalimnus Ribaut, 1948

Micrelloides Evans, 1973 placement nov. (previously unplaced in Paralimnini)

Micropedeticus Stiller, 2009 placement nov. (previously unplaced in Paralimnini)

Miraldus Lindberg, 1960 placement nov. (previously unplaced in Paralimnini)

Mocuola Emeljanov, 1964 placement nov. (previously unplaced in Paralimnini)

Mogangella Dlabola, 1957 placement nov. (previously unplaced in Paralimnini)

Mogangina Emeljanov, 1962 placement nov. (previously unplaced in Paralimnini)

Mongolojassus Zachvatkin, 1953 placement nov. (previously unplaced in Paralimnini)

Multiproductus Xing, Dai \& Li, 2011 placement nov. (previously unplaced in Paralimnini)

Myittana Distant, 1908 placement nov. (previously unplaced in Paralimnini)

Nanosius Dlabola, 1974 placement nov. (previously unplaced in Paralimnini)

Naudeus Theron, 1982 placement nov. (previously unplaced in Paralimnini)

Nicolaus Lindberg, 1958 placement nov. (previously unplaced in Paralimnini)

Orocastus Oman, 1949 placement nov. (previously unplaced in Paralimnini)

Pantallus Emeljanov, 1961

Paraglena Emeljanov, 1997

Paragygrus Emeljanov, 1964 placement nov. (previously unplaced in Paralimnini)

Paralaevicephalus Ishihara, 1953 placement nov. (previously unplaced in Paralimnini)

Paralimnellus Emeljanov, 1972 placement nov. (previously unplaced in Paralimnini)

Paralimnoidella Kwon \& Lee, 1979 placement nov. (previously unplaced in Paralimnini)

Paralimnus Matsumura, 1902

Paramesanus Dlabola, 1979 placement nov. (previously unplaced in Paralimnini)

Paramesus Fieber, 1866

Parapotes Emeljanov, 1975

Pasaremus Oman, 1949 placement nov. (transferred from Cicadulini)

Pazu Oman, 1949 placement nov. (previously unplaced in Paralimnini)

Peconus Oman, 1949 placement nov. (previously unplaced in Paralimnini)

Philaia Dlabola, 1952 placement nov. (previously unplaced in Paralimnini)

Phlebiastes Emeljanov, 1961 placement nov. (previously unplaced in Paralimnini).

Pinumius Ribaut, 1946 placement nov. (previously unplaced in Paralimnini) 
ZAHNISER J.N. \& DIETRICH C.H., A review of the tribes of Deltocephalinae (Cicadellidae)

Platentomus Theron, 1980 placement nov. (previously unplaced in Paralimnini) Pleargus Emeljanov, 1964 placement nov. (previously unplaced in Paralimnini) Praganus Dlabola, 1949 placement nov. (previously unplaced in Paralimnini) Pravistylus Theron, 1975 placement nov. (previously unplaced in Paralimnini) Prosperellus Emeljanov, 1999 placement nov. (previously unplaced in Paralimnini)

Psammotettix Haupt, 1929

Pseudolausulus Wagner \& Franz, 1961 placement nov. (previously unplaced in Paralimnini)

Pteropyx Haupt, 1927 placement nov. (previously unplaced in Paralimnini)

Quartausius Dlabola, 1974 placement nov. (previously unplaced in Paralimnini)

Quaziptus Kramer, 1965 placement nov. (previously unplaced in Paralimnini)

Restiobia Davies, 1988 placement nov. (previously unplaced in Paralimnini)

Rhoananus Dlabola, 1949

Rodezotettix Della Giustina \& Wilson, 1995 placement nov. (previously unplaced in Paralimnini)

Rosenus Oman, 1949 placement nov. (previously unplaced in Paralimnini)

Samuraba Linnavuori, 1961 placement nov. (previously unplaced in Paralimnini)

Savanicus Dlabola, 1977 placement nov. (previously unplaced in Paralimnini)

Secopennis DeLong \& Sleesman, 1929 placement nov. (previously unplaced in Paralimnini)

Sestrelicola Remane \& Asche, 1980 placement nov. (previously unplaced in Paralimnini)

Sicistella Emeljanov, 1972 placement nov. (previously unplaced in Paralimnini)

Soracte Kirkaldy, 1907 placement nov. (previously placed in Platymetopiina)

Soractellus Evans, 1966 placement nov. (transferred from Deltocephalini)

Sorhoanus Ribaut, 1946

Spartopyge Young \& Beirne, 1958 placement nov. (previously unplaced in Paralimnini)

Subhimalus Ghauri, 1971 placement nov. (previously unplaced in Paralimnini)

Takagiella Vilbaste, 1969 placement nov. (previously unplaced in Paralimnini)

Teinopterus Stiller, 2011 placement nov. (previously unplaced in Paralimnini)

Telusus Oman, 1949 placement nov. (previously unplaced in Paralimnini)

Tiaratus Emeljanov, 1961 placement nov. (previously unplaced in Paralimnini)

Tigriculus Dlabola, 1961 placement nov. (previously unplaced in Paralimnini)

Triasargus Novikov \& Anufriev, 2005 placement nov. (previously unplaced in Paralimnini)

Tytthuspilus Stiller, 2011 placement nov. (previously unplaced in Paralimnini)

Umeqi Stiller, 2011 placement nov. (previously unplaced in Paralimnini)

Urganus Dlabola, 1965 placement nov. (previously unplaced in Paralimnini)

Vecaulis Theron, 1975 placement nov. (previously unplaced in Paralimnini)

Verdanus Oman, 1949

Vilargus Theron, 1975 placement nov. (previously unplaced in Paralimnini)

Yanocephalus Ishihara, 1953 placement nov. (previously unplaced in Paralimnini)

Pendarini Dmitriev, 2009

Fig. 44

Type genus: Pendarus Ball, 1927.

\section{Diagnosis}

Pendarini are medium sized to large ochraceous, yellowish, greenish, or brownish leafhoppers, sometimes with brown irrorate markings or ramose pigment lines. As nymphs, they are distinguished from other Deltocephalinae by the carinate crown-face transition, face with medial longitudinal carina in upper part, acrometope with anterior margin tracing anterior carina of head, abdomen with 4-6 longitudinal rows of very short macrosetae, and pygofer elongate (Dmitriev, 2009). Adults often have male pygofer 
with an acute apex and with one or more spines or teeth, and the aedeagus often with single or paired apical or preapical processes.

\section{Description}

Head. Head subequal to or wider than pronotum. Discal portion of crown glabrous with radial or longitudinal striae, or completely shagreen to base. Anterior margin of head shagreen or foliaceous. Frontoclypeus not tumid; texture shagreen. Clypellus parallel-sided or widening apically; apex following or slightly surpassing normal curve of gena. Lorum subequal to or wider than clypellus near base. Antennal bases near middle or posteroventral (lower) corners of eyes. Antennae short, less than $1.5 \mathrm{x}$ width of head. Gena obtusely incised laterally; with fine erect seta beside laterofrontal suture. Antennal ledges absent or weakly developed (carinate or weakly carinate). Ocelli present; close to eyes; on anterior margin of head.

THORAX. Pronotum lateral margin carinate; lateral margin shorter than half basal width of eye.

WiNGS. Forewing macropterous or submacropterous; appendix present and restricted to anal margin or rarely absent or reduced (Dorydiella); with 3 anteapical cells; veins not raised; without reflexed costal veins; A1-A2 crossvein absent or present; apical venation not highly reticulate.

Legs. Profemur with AM1 seta only; intercalary row with one row of five or more fine setae; row AV with short, stout setae. Protibia dorsal surface rounded, convex. Metafemur apex macrosetae $2+2+1$. Metatarsomere I not expanded apically; plantar setae simple, tapered.

MaLe Genitalia. Valve articulated with pygofer; lateral margin short, articulating with pygofer at a point. Pygofer basolateral membranous cleft present; macrosetae well differentiated into several rows; sometimes elongate or extended posteriorly; often with one or more spines or processes arising dorsally or ventrally. Subgenital plates free from each other; articulated with valve; macrosetae uniseriate laterally. Style broadly bilobed basally; median anterior lobe pronounced. Aedeagus with single shaft and gonopore. Connective anterior arms somewhat divergent, $\mathbf{Y}$ - or $\mathbf{U}$-shaped; stem shorter than arms; articulated with aedeagus.

Female genitalia. Pygofer with numerous macrosetae. Ovipositor not protruding far beyond pygofer apex. First valvula convex; dorsal sculpturing pattern strigate, granulose, or maculose; sculpturing reaching dorsal margin; without distinctly delimited ventroapical sculpturing. Second valvula broad, gradually tapered; with or without dorsal median tooth; teeth on apical 1/3 or more; teeth small, regularly or irregularly shaped.

\section{Geography and ecology}

Distribution: Nearctic and Neotropical regions. Species of this tribe feed on a variety of plants including grasses, herbs, and woody hosts, including conifers. Several species apparently alternate between woody oviposition hosts and herbaceous food plants. Some Pendarus and Paraphlepsius species specialize on single species of perennial grasses. Chlorotettix species feed on grasses and sedges.

\section{Remarks}

Pendarini was recently described by Dmitriev (2009) based on nymphal characters and contains 8 genera and 249 species. Five genera (Bandaromimus, Tropicanus, Ileopeltus, Chlorotettix and Copididonus) are added to the tribe here based on the results of our phylogenetic analyses and based on observations of nymphs of Chlorotettix which share the elongate pygofer of other Pendarini. A clade including representatives of Paraphlepsius Baker, 1897, Dorydiella Baker, 1897, Bandaromimus Linnavuori, 1959, Tropicanus DeLong, 1944, Chlorotettix, and Copididonus was consistently resolved in all analyses 


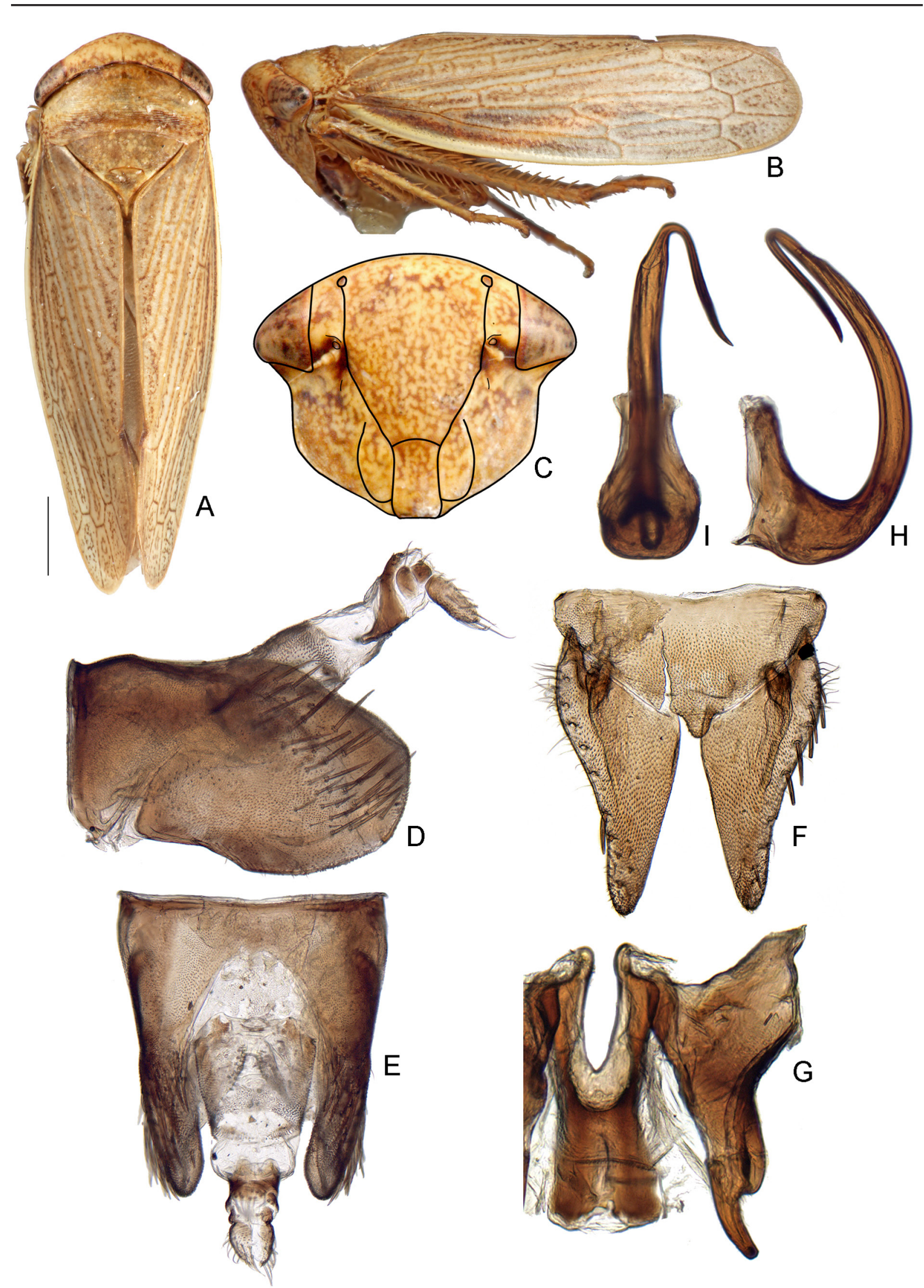

Fig. 44. Pendarini. Pendarus magnus (Osborn \& Ball, 1897). A-I. Standard views (see Material \& Methods). 
and received moderate branch support in Bayesian analyses. Pendarus was not included in the analyses but is undoubtedly related to this group. Ileopeltus is included based on the comments of Cwikla (1988b) suggesting the genus is closely related to Chlorotettix. Further study is needed to determine if other genera should be placed in this tribe and to discover additional morphological characters to aid in its diagnosis. The tribe is an exclusively New World group as currently understood.

\section{Selected references}

DeLong (1945), Hamilton (1975a), Cwikla (1988a, b), Dmitriev (2009).

\section{Included genera}

Bandaromimus Linnavuori, 1959 placement nov. (transferred from Athysanini)

Chlorotettix Van Duzee, 1892 placement nov. (transferred from Athysanini)

Copididonus Linnavuori, 1954 placement nov. (transferred from Athysanini)

Dorydiella Baker, 1897

Ileopeltus Cwikla, 1988 placement nov. (previously unplaced in Deltocephalinae)

Paraphlepsius Baker, 1897

Pendarus Ball, 1927

Tropicanus DeLong, 1944 placement nov. (transferred from Athysanini)

Penthimiini Kirschbaum, 1868

Fig. 45

Type genus: Penthimia Germar, 1821.

$=$ Thaumatoscopini Baker, 1923.

\section{Diagnosis}

Penthimiini are medium sized to large, squat, robust, often black or brown leafhoppers; often with ventral part of face and/or entire ventral side flattened and dorsal side convex. They can be identified by the ocelli on the crown and often distant from the eyes, strong antennal ledge, dorsally flattened and carinate protibia, and forewing with appendix large and extending around the wing apex.

\section{Description}

HEAD. Head subequal to, wider than, or distinctly narrower pronotum. Discal portion of crown glabrous with radial or longitudinal striae. Anterior margin of head with numerous transverse striations or carinae, or foliaceous. Head and pronotum usually sloping anteriorly. Face short, broad. Frontoclypeus tumid or not; texture striate or glabrous. Clypellus widening apically; apex following or slightly surpassing normal curve of gena. Lorum subequal to or wider than clypellus near base. Antennal bases near upper or anterodorsal corners of eyes. Antennae short, less than $1.5 \mathrm{x}$ width of head. Gena obtusely incised laterally; with fine erect seta beside laterofrontal suture. Antennal ledges strongly developed (with a distinct ledge); sometimes forming lateral part of anterior margin of head. Ocelli present; distant from eyes; on crown.

THORAx. Pronotum lateral margin carinate; lateral margin shorter or longer than basal width of eye.

Wings. Forewing macropterous or submacropterous; sometimes coriaceous, setose, punctate, or tuberculate; appendix large and extending around wing apex or rarely restricted to anal margin; with 3 anteapical cells; veins not raised; without reflexed costal veins; A1-A2 crossvein absent or present; apical venation not highly reticulate. 
Legs. Profemur with AM1 seta only or with AM1 and with one or more additional proximal setae; intercalary row with one row of five or more fine setae; row AV usually with thin, hair-like setae or without setae, more rarely with short, stout setae or with relatively long macrosetae. Protibia dorsal surface flat, $\mathrm{AD}$ and $\mathrm{PD}$ margins sharply carinate or at $\sim 90^{\circ}$ (not rounded). Metafemur apex macrosetae $2+2+1$. Metatarsomere I not expanded apically, plantar setae simple, tapered.

MaLe Genitalia. Valve articulated with pygofer; lateral margin short, articulating with pygofer at a point. Pygofer basolateral membranous cleft present; macrosetae well differentiated into several rows. Subgenital plates free from each other; articulated with valve; without macrosetae or macrosetae scattered, irregularly arranged. Style linear, median anterior lobe not pronounced or broadly bilobed basally, median anterior lobe pronounced. Basal processes of the aedeagus/connective absent or reduced or present, connected or articulated to connective or near base of aedeagus. Aedeagus with single shaft and gonopore. Connective anterior arms somewhat divergent, $\mathbf{Y}$ - or $\mathbf{U}$-shaped; articulated with aedeagus.

Female genitalia. Pygofer with numerous macrosetae. Ovipositor not protruding or rarely protruding (e.g., Penthimidia) far beyond pygofer apex. First valvula convex; dorsal sculpturing pattern strigate, concatenate, or reticulate; sculpturing reaching dorsal margin; without distinctly delimited ventroapical sculpturing. Second valvula abruptly broadened medially or subapically or broad, gradually tapered; with or without dorsal median tooth; teeth on apical 1/3 or more; teeth large, regularly shaped or small, regularly or irregularly shaped.

\section{Geography and ecology}

Distribution: cosmopolitan, poorly represented in North America. Penthimiini are collected on trees, shrubs, and herbaceous vegetation.

\section{Remarks}

Penthimiini contains 46 genera and 203 species. It is a relatively early diverging lineage of Deltocephalinae but has the typical derived deltocephaline characters of the male genitalia (pygofer with basolateral membranous cleft, $\mathbf{Y}$-shaped connective, and style broadly bilobed basally). The phylogenetic analyses included 4 exemplars of the tribe as circumscribed here (Penthimia, Penthimidia, Penthimiola, and Jafar included in analyses; Citorus Stål, 1866 is removed from the tribe based on these analyses). Parsimony analyses resolved the former 3 exemplars as monophyletic and sister to Magnentius Singh-Pruthi, 1930, while ML and Bayesian analyses resolved Penthimidia and Penthimiola together and Penthimia in an uncertain position elsewhere in the tree. It is unclear why Penthimia does not always group with other penthimiines in phylogenetic analyses, but based on the morphological evidence supporting Penthimiini as a taxon, we suspect that the phylogenetic placement of Penthimia in the ML and Bayesian analyses is artifactual. Jafar was also included for the first time and grouped with one or two undescribed African genera, somewhat distant from other Penthimiini but with no branch support. Because its phylogenetic position is uncertain, and because it possesses several characters diagnostic for the tribe (large and robust body, ocelli on crown, inflated frontoclypeus, strong antennal ledges, and dorsally flattened and bicarinate protibiae), it is retained in the tribe pending further study.

Citorus, which was previously included in Penthimiini based on its body shape and large forewing appendix, was found with strong branch support to be more closely related to Selenocephalini (Dwightla and Selenocephalus). Based on the phylogenetic results and on some morphological characters that differ from Penthimiini (anterior margin of the head with distinct sharp carinae, ocelli on anterior margin of head and close to eyes, antennal ledge not very strong or ledge-like) it is transferred to Selenocephalina here. The squat, robust body form and large appendix are similar to Penthimiini but these are apparently convergent characters in this case. 


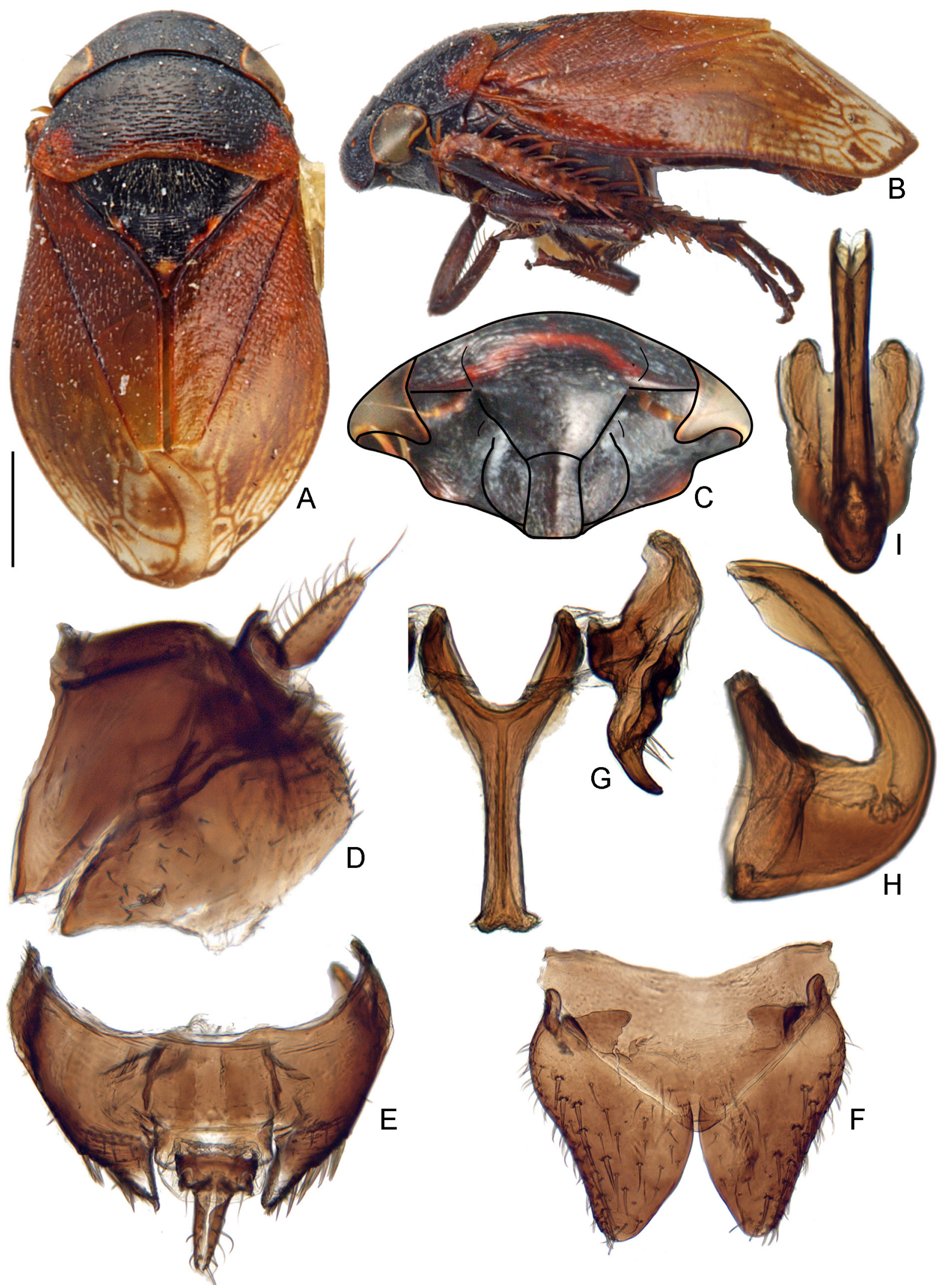

Fig. 45. Penthimiini. Penthimia americana Fitch, 1851. A-I. Standard views (see Material \& Methods). 


\section{Selected references}

Evans (1966, 1972), Linnavuori (1977).

\section{Included genera}

Alopenthimia Evans, 1972

Amberbakia Distant, 1912

Chanohirata Hayashi \& Machida, 1996

Chinaella Evans, 1935

Ectopiocephalus Kirkaldy, 1906

Eovulturnops Evans, 1947

Eupenthimia Evans, 1972

Eusallya Evans, 1972

Foroa Linnavuori, 1977

Gressittella Evans, 1972

Haranga Distant, 1908

Irenaella Linnavuori, 1977

Jafar Kirkaldy, 1903

Kronos Distant, 1917

Ledroides Dammerman, 1910

Macutella Evans, 1972

Malichus Distant, 1918

Megalopenthimia Evans, 1954

Musosa Linnavuori, 1977

Neodartellus Evans, 1955

Neodartus Melichar, 1903

Neopenthimia Evans, 1972

Neovulturnus Evans, 1937

Nielsoniella Linnavuori, 1977

Nortoides Evans, 1972

Nubelella Evans, 1972

Nubelloides Evans, 1972

Osella Evans, 1972

Penthimia Germar, 1821

Penthimidia Haglund, 1899

Penthimiella Evans, 1972

Penthimiola Linnavuori, 1959

Penthimiopsis Evans, 1972

Pentria Evans, 1972

Piorella Evans, 1972

Platyscopus Evans, 1941

Reticuluma Cheng \& Li, 2005

Sidelloides Evans, 1972

Tambila Distant, 1908

Thaumatopoides Evans, 1947

Thaumatoscopus Kirkaldy, 1906

Tolasella Evans, 1972

Tomaloides Evans, 1972

Uzelina Melichar, 1903

Vertigella Evans, 1972

Vulturnus Kirkaldy, 1906 


\section{Phlepsiini tribe nov. \\ urn:1sid:zoobank.org:act:6F2DC22C-BD71-4BFD-BEEE-8AF8BA2819B4}

Fig. 46

Type genus: Phlepsius Fieber, 1866.

\section{Diagnosis}

Phlepsiini are medium sized to large, robust, ivory to brown or dark brown leafhoppers, often with brown irrorate markings on the forewing and body. They can be identified by the head distinctly narrower than pronotum, antennal ledges carinate, lorum long and relatively large, clypellus long and widening apically, pronotum lateral margin longer than half basal width of eye, profemur row AM sometimes with 1 or more setae basad of AM1, and nymph with short pygofer and with 6 rows of abdominal macrosetae.

\section{Description}

HEAD. Head narrower than pronotum. Discal portion of crown glabrous with radial or longitudinal striae. Anterior margin of head with one to several transverse carinae or striae, irregularly textured, or partly glabrous. Frontoclypeus not tumid; not particularly broad; texture shagreen. Clypellus widening apically; relatively long; apex following or slightly surpassing normal curve of gena. Lorum large; subequal to or wider than clypellus near base. Antennal bases near middle or posteroventral (lower) corners of eyes. Antennae short, less than $1.5 \mathrm{x}$ width of head. Gena obtusely incised laterally; with fine erect seta beside laterofrontal suture. Antennal ledges weakly developed (carinate or weakly carinate). Ocelli present; close to eyes; on anterior margin of head.

THORAX. Pronotum lateral margin carinate; lateral margin longer than half basal width of eye, sometimes longer than entire basal width of eye.

Wings. Forewing macropterous; appendix restricted to anal margin; with 3 anteapical cells; veins not raised; A1-A2 crossvein absent or present; apical venation not highly reticulate; with numerous false veins and irrorate markings.

LEGS. Profemur with AM1 seta only or rarely with extra macrosetae basad of AM1; intercalary row with one row of five or more fine setae; row AV with short, stout setae or with longer setae. Protibia dorsal surface rounded, convex; dorsal macrosetae $4+4$. Metafemur apex macrosetae $2+2+1$. Metatarsomere I not expanded apically; plantar setae simple, tapered.

Male Genitalia. Valve articulated with pygofer; lateral margin short, articulating with pygofer at a point. Pygofer basolateral membranous cleft present; macrosetae well differentiated into several rows; without spines or processes. Subgenital plates free from each other; articulated with valve; macrosetae uniseriate laterally. Style broadly bilobed basally; median anterior lobe pronounced. Basal processes of the aedeagus/connective absent or reduced, or present, connected or articulated to connective or near base of aedeagus. Aedeagus with single shaft and gonopore. Connective anterior arms somewhat divergent, Y-shaped; articulated with aedeagus.

Female Genitalia. Pygofer with numerous macrosetae. Ovipositor not protruding far beyond pygofer apex. First valvula convex; dorsal sculpturing pattern strigate, reticulate, or concatenate; sculpturing reaching dorsal margin; without distinctly delimited ventroapical sculpturing. Second valvula broad, gradually tapered; without dorsal median tooth; teeth on apical $1 / 3$ or more; teeth small, regularly or irregularly shaped. 


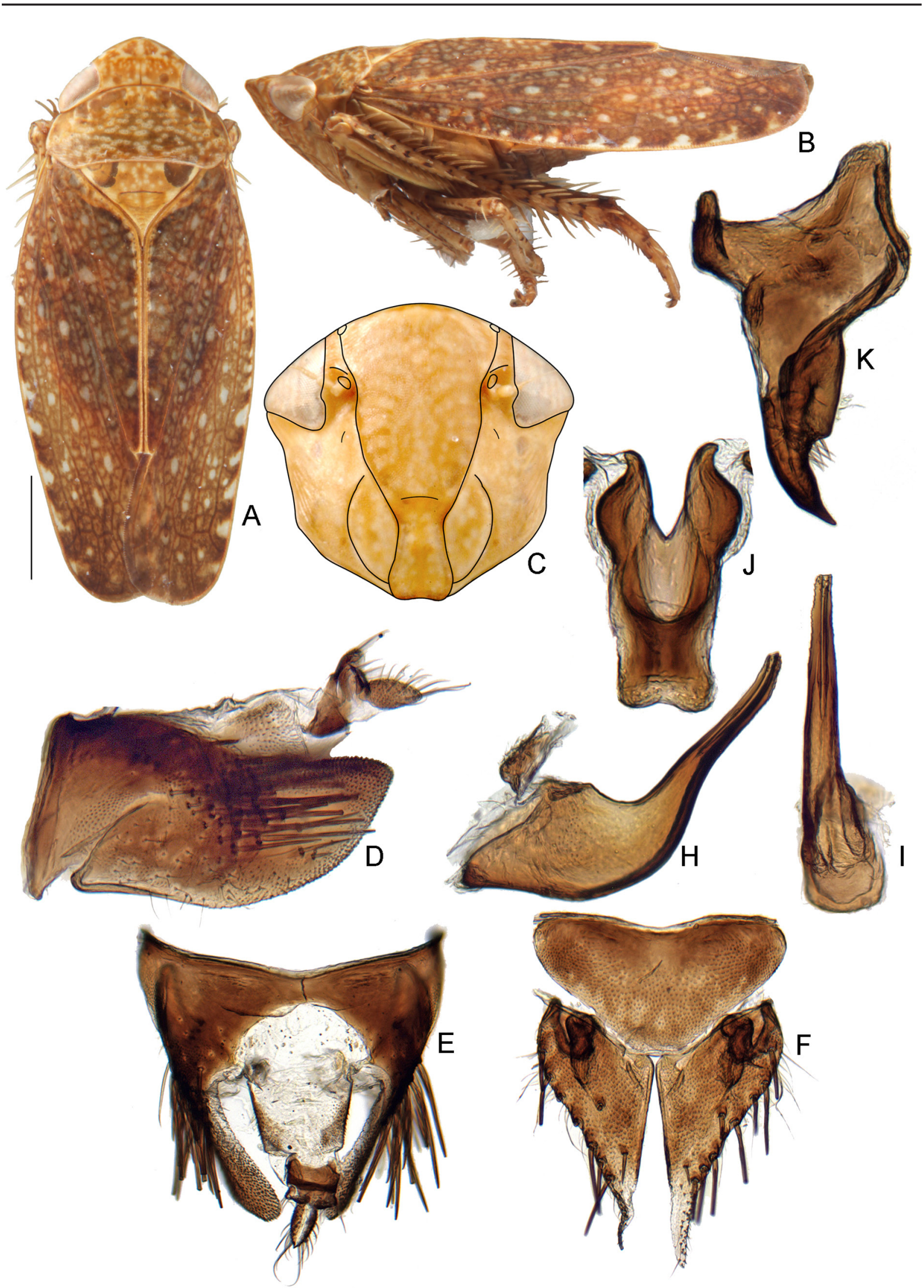

Fig. 46. Phlepsiini. Phlepsius intricatus (Herrich-Schäffer, 1838). A-F, H-I. Standard views (see Material \& Methods). J. Connective, ventral view. K. Style, ventral view. 


\section{Geography and ecology}

Distribution: Palearctic, Afrotropical, Oriental, Nearctic, and Neotropical regions. Phlepsiini feed on woody or herbaceous dicots. Texananus lathropi (Baker, 1925), T. latipex DeLong 1943, T. oregonus (Ball, 1931), T. pergradus DeLong, 1938 and T. spatulatus (Van Duzee, 1892) are vectors of the western strain of North American aster yellows.

\section{Remarks}

Phlepsiini currently only includes 4 genera and 81 species. Phylogenetic analyses identified a wellsupported clade including Phlepsius, Excultanus, and Korana which was consistently found in a clade along with Scaphoideini, Drabescini, and some undescribed genera. Oman (1941) recognized a tribe, "Phlepsiini", which is considered here to be a nomen nudum (see Materials and Methods: Taxonomic Treatments, above). In addition to Phlepsius (which included the species of Texananus and Excultanus), Oman included Norvellina, Remadosus, Dorydiella, Dixianus, Paraphlepsius, and Phlepsanus. The phylogenetic analyses here do not support this broad interpretation of the tribe. Orientus has several characters diagnostic for this tribe but is smaller and more slender than the other genera. It was also not placed within the Phlepsiini clade in the phylogenetic analyses, but was found on a long branch of indeterminate relationship near the early diverging lineages of Deltocephalinae. It is retained in Athysanini at this time pending further analyses.

\section{Selected references}

DeLong (1938, 1939), Crowder (1952), Theron (1980), McKamey (2000, 2003a).

\section{Included genera}

Excultanus Oman, 1949 placement nov. (previously unplaced in Athysanini)

Korana Distant, 1910 placement nov. (previously unplaced in Deltocephalinae)

Phlepsius Fieber, 1866 placement nov. (previously placed in Platymetopiina)

Texananus Ball, 1918 placement nov. (previously placed in Platymetopiina)

\section{Scaphoideini Oman, 1943}

Fig. 47

Type genus: Scaphoideus Uhler, 1889.

\section{Diagnosis}

Scaphoideini are medium sized (usually 4-7 mm), often slender, ivory, yellowish, ochraceous, or brown leafhoppers, often marked with orange, brown, or black on head, pronotum, or forewings. None of the following diagnostic characters are present in all taxa, but some combination of these characters is present in all and a few $\left(^{*}\right)$ appear to be unique to this tribe: head narrower than pronotum, produced; frontoclypeus long, narrow; antennae long; body slender; head and wings often with brown, orange, ochraceous, or ivory markings; forewing with one or more darkly pigmented reflexed veins in vicinity of outer anteapical cell; profemur row AV setae absent or reduced (without stout setae); metatibia macrosetae in row PD long, as long as or longer than $0.5 \mathrm{x}$ length of protibia*; male or female pygofer with dense tufts of long fine or regular setae*; subgenital plate apex membranous or long, digitate, and somewhat membranous or weakly sclerotized; subgenital plates with long fine setae laterally and/or dorsally (also occurs in many other deltocephaline tribes); basal processes of the aedeagus or connective sometimes present, connected or articulated to base of aedeagus or apex of connective stem. 


\section{Description}

HEAD. Head often narrower than pronotum, sometimes subequal to or wider than pronotum; anterior margin shagreen; crown rounded to face or angulate. Discal portion of crown glabrous with radial or longitudinal striae. Frontoclypeus not tumid; long, narrow; shagreen. Clypellus widening apically. Lorum subequal to or wider than clypellus near base. Antennal bases near middle or posteroventral (lower) corners of eyes or near anterodorsal (upper) corners of eyes. Antennae long (longer than width of head). Gena obtusely incised or not incised laterally; with fine erect seta beside laterofrontal suture. Antennal ledge absent. Ocelli close to eyes.

THORAx. Pronotum lateral margin carinate; lateral margin shorter or longer than half basal width of eye.

WINGS. Forewing macropterous; appendix restricted to anal margin; veins sometimes bordered or highlighted at apices with brown coloration; with 3 anteapical cells; often with pigmented reflexed costal veins; usually without A1-A2 crossvein; apical venation not highly reticulate.

Legs. Profemur row AM with AM1 seta only; intercalary row with one row of five or more fine setae; row AV setae usually reduced or absent, or sometimes short, stout setae. Protibia dorsal surface rounded, convex. Metatibia macrosetae in rows AD and PD often very long, row PD setae often equal to or longer than $0.5 \mathrm{x}$ length of protibia. Metatarsomere I long. Metafemur apical setae $2+2+1$.

MaLE Genitalia. Valve articulated with pygofer; lateral margin short, articulating with pygofer at a point. Pygofer with basolateral membranous cleft; often with many long thick or fine setae sometimes arranged in dense tufts or groups. Subgenital plate with macrosetae absent, uniseriate laterally, or uniseriate, distant from lateral margin; often with long fine hairs laterally and/or dorsally; plate apex often long, digitate, and/or membranous or filamentous. Style broadly bilobed basally, median anterior lobe pronounced. Basal processes of the aedeagus/connective absent or present, connected to or articulated with connective or near base of aedeagus. Aedeagus with single shaft and gonopore. Connective usually $\mathbf{Y}$-shaped, sometimes linear or with anterior arms closely appressed; articulated or rarely fused (Sikhamani, Thryaksha, and Univagris) with aedeagus, or aedeagus dissociated from connective (Scaphoideus).

Female genitalia. Pygofer with numerous macrosetae, sometimes with dense tufts or groups of fine or thick setae. Ovipositor not protruding far beyond pygofer apex. First valvula convex; dorsal sculpturing pattern often concatenate, sometimes strigate or reticulate; reaching dorsal margin. First valvula ventroapical sculpturing indistinctly delimited or absent. Second valvula abruptly broadened medially or subapically or broad, gradually tapered; with or without dorsal median tooth; dorsoapical teeth large or small, regularly shaped; teeth on apical $1 / 3$ or more.

\section{Geography and ecology}

Distribution: cosmopolitan. Scaphoideini are among the most commonly encountered forest-dwelling deltocephalines throughout the tropical and warm-temperate regions of the world. Nymphs and adults are often collected on understory grasses or herbaceous vegetation but most species appear to require woody hosts for oviposition. Scaphoideus titanus Ball, 1932 is a serious pest and vector of grape flavescence dorée in Europe and of chrysanthemum yellows. S. luteolus Van Duzee, 1894 is a vector of elm phloem necrosis in the United States. Osbornellus borealis DeLong \& Mohr, 1936 is a vector of western $\mathrm{X}$ disease.

\section{Remarks}

Scaphoideini contains 61 genera and 631 species. Oman et al. (1990) included 12 genera, significantly fewer than the circumscription here. Several genera included previously (Acunasus DeLong, 1945, 


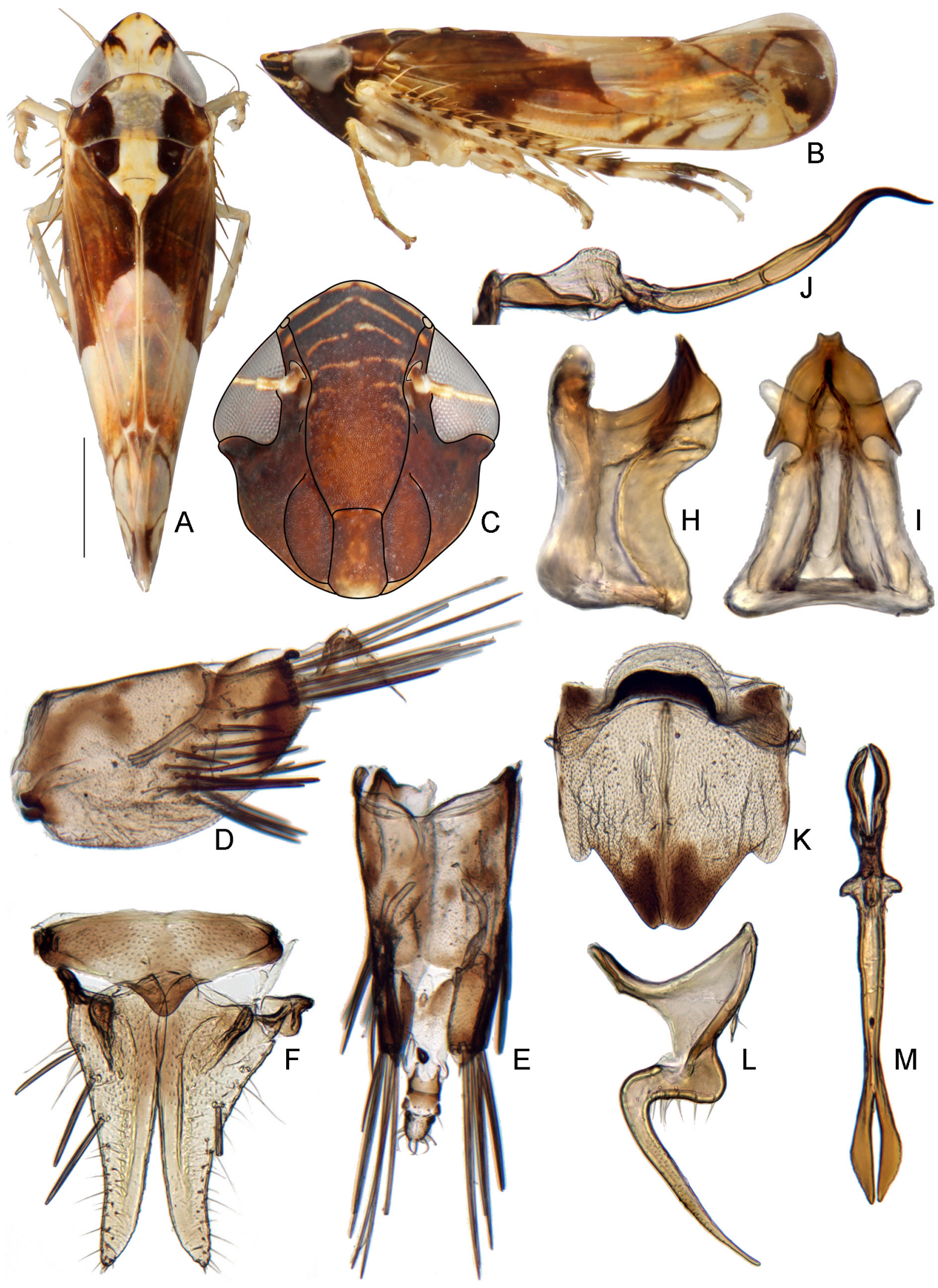

Fig. 47. Scaphoideini. Scaphoideus omani sp. nov. A-F, H-I. Standard views (see Material \& Methods). J. Lateral view of connective and connective processes. K. $q$ sternite VII. L. Style, ventrally. M. Ventral view of connective and connective processes. 
Danbara Oman, 1949, Sobara Oman, 1949 and Twiningia Ball, 1931) are not included here. Twiningia was included in the phylogenetic analyses here and was found to be closely related to Platymetopius Burmeister, 1838 (Athysanini), and the others are excluded because they do not possess characters considered here to be diagnostic for the tribe.

The phylogenetic analyses presented here consistently resolved a clade including Scaphoideini sensu novo plus Drabescini. This clade was in turn resolved as sister to Phlepsiini. Scaphoideini was resolved as paraphyletic with respect to Drabescini and three undescribed genera, but with little or no branch support for the basal branches of the group. Genera of Scaphoideini included in phylogenetic analyses here that were not included previously are Loipothea Linnavuori, 1969, Omanana DeLong, 1942, Scaphoidophyes Kirkaldy, 1906, Anoplotettix Ribaut, 1942 and Mimotettix Matsumura, 1914. Further taxon sampling and molecular data may help to resolve the relationships within this large tribe and its relationship to the other related tribes, but the available evidence suggests that this is a lineage distinct from other Athysanini, the tribe in which many of the genera were previously placed. The circumscription of the tribe here is based on the phylogenetic analyses and the morphological characters identified here as diagnostic for most members of the group. Representatives of most genera of Athysanini were examined or were reviewed from the literature for their potential placement in Scaphoideini. Further adjustments to the circumscription may be needed after more detailed study of the group.

The Perugrampta group (including Perugramptella, Penthigrampta, and Stenogrampta) is placed here pending further study. These genera possess the following characters that are diagnostic for Scaphoideini: profemur row AV setae absent, darkly pigmented reflexed branches of $\mathrm{R}$ vein, male or female pygofer or subgenital plates with tufts of fine or thick setae, subgenital plates with long fine setae laterally, and basal processes of the aedeagus or connective present and connected to base of aedeagus.

\section{Selected references}

Barnett (1977, 1979, 1980), Martinson (1977), Knight \& Fletcher (2007), Viraktamath \& Mohan (1994, 2004), Stiller (2001), Zhang \& Dai (2006), Freytag (2008), Dominguez \& Godoy (2010), Dai \& Dietrich (2011).

\section{Included genera}

Acastroma Linnavuori, 1969 placement nov. (transferred from Athysanini) Afroideus Linnavuori, 1961 placement nov. (previously unplaced in Deltocephalinae) Alemaia Heller \& Linnavuori, 1968 placement nov. (previously unplaced in Deltocephalinae) Amimenus Ishihara, 1953 placement nov. (previously unplaced in Deltocephalinae) Anoplotettix Ribaut, 1942 placement nov. (previously placed in Platymetopiina) Bampurius Dlabola, 1977 placement nov. (transferred from Athysanini) Banus Distant, 1908 placement nov. (transferred from Athysanini) Burakia Kocak, 1981 placement nov. (previously placed in Platymetopiina) Cantura Oman, 1949 placement nov. (previously unplaced in Deltocephalinae) Caphodus Oman, 1938 placement nov. (previously unplaced in Deltocephalinae) Capoideus Theron, 1974 placement nov. (previously unplaced in Deltocephalinae) Coroticus Distant, 1918 placement nov. (transferred from Scaphytopiini) Dariena Linnavuori \& DeLong, 1977 placement nov. (transferred from Athysanini) Delospilopterus Stiller, 2001 placement nov. (transferred from Athysanini) Euscelidella Evans, 1954 placement nov. (previously unplaced in Deltocephalinae) Ferganotettix Dubovsky, 1966 placement nov. (transferred from Athysanini) Grammacephalus Haupt, 1929 placement nov. (transferred from Scaphytopiini) Gunghuyana Distant, 1910 placement nov. (previously unplaced in Deltocephalinae) Loipothea Linnavuori, 1969 placement nov. (previously unplaced in Deltocephalinae) 
Longicornis Li \& Song, 2008 placement nov. (transferred from Athysanini) Madagella Evans, 1954 placement nov. (previously placed in Platymetopiina) Melanetettix Knight \& Fletcher, 2007 placement nov. (transferred from Athysanini) Mesotettix Matsumura, 1914 placement nov. (previously unplaced in Deltocephalinae) Mimotettix Matsumura, 1914 placement nov. (transferred from Athysanini) Mirzayansus Dlabola, 1979 placement nov. (transferred from Athysanini) Monobazus Distant, 1908 placement nov. (transferred from Athysanini) Moorada Ghauri, 1975 placement nov. (transferred from Athysanini) Nataretus Theron, 1980 placement nov. (transferred from Athysanini) Neolimnus Linnavuori, 1953 placement nov. (transferred from Athysanini) Neophlepsius Linnavuori, 1955 placement nov. (transferred from Athysanini) Omanana DeLong, 1942 placement nov. (transferred from Athysanini) Osbornellus Ball, 1932 placement nov. (transferred from Athysanini) Pachodus Linnavuori, 1961 placement nov. (transferred from Athysanini) Parallygus Melichar, 1903 placement nov. (transferred from Athysanini) Paramelia Evans, 1954 placement nov. (previously placed in Platymetopiina) Penthigrampta Dietrich \& Rakitov, 2002 placement nov. (transferred from Penthimiini) Perugrampta Kramer, 1965 placement nov. (transferred from Penthimiini) Perugramptella Dietrich \& Rakitov, 2002 placement nov. (transferred from Penthimiini) Phlogotettix Ribaut, 1942 placement nov. (previously unplaced in Deltocephalinae) Phlogothamnus Ishihara, 1961 placement nov. (previously unplaced in Deltocephalinae) Platyretus Melichar, 1903 placement nov. (transferred from Athysanini) Premanus DeLong, 1944 placement nov. (transferred from Athysanini) Prescottia Ball, 1932 placement nov. (previously unplaced in Deltocephalinae) Scaphodhara Viraktamath \& Mohan, 1994 placement nov. (previously unplaced in Deltocephalinae) Scaphoidella Vilbaste, 1968 placement nov. (transferred from Athysanini)

Scaphoideus Uhler, 1889 placement nov. (transferred from Athysanini) Scaphoidophyes Kirkaldy, 1906 placement nov. (previously unplaced in Deltocephalinae) Scaphoidula Osborn, 1923 placement nov. (previously unplaced in Deltocephalinae) Scaphomonus Viraktamath, 2009 placement nov. (transferred from Athysanini) Sikhamani Viraktamath \& Webb, 2006 placement nov. (transferred from Scaphytopiini) Soleatus DeLong, 1971 placement nov. (previously unplaced in Deltocephalinae) Spathanus DeLong, 1945 placement nov. (transferred from Athysanini) Stenogrampta Dietrich \& Rakitov, 2002 placement nov. (transferred from Penthimiini) Sudhamruta Viraktamath \& Anantha Murthy, 1999 placement nov. (transferred from Scaphytopiini) Tbilisica Dlabola, 1958 placement nov. (previously placed in Platymetopiina) Thamnophryne Kirkaldy, 1907 placement nov. (previously unplaced in Deltocephalinae) Thryaksha Viraktamath \& Anantha Murthy, 1999 placement nov. (transferred from Scaphytopiini) Tongdotettix Kwon, 1980 placement nov. (previously placed in Platymetopiina) Tuakamara Webb, 1980 placement nov. (previously placed in Platymetopiina) Univagris Viraktamath \& Anantha Murthy, 1999 placement nov. (transferred from Scaphytopiini) Wanritettix Vilbaste, 1969 placement nov. (transferred from Athysanini)

Scaphytopiini Oman, 1943

Fig. 48

Type genus: Scaphytopius Ball, 1931. 


\section{Diagnosis}

Scaphytopiini are small to medium sized usually brownish, or sometimes black, yellow, or greenish leafhoppers, sometimes with irrorate markings and often with reflexed costal veins on the forewing. They can be identified by the anteriorly produced and pointed head, gena not incised laterally and visible behind eyes in dorsal view, frontoclypeus elongate, reflexed costal veins on the forewing, valve large, and connective broad, $\mathbf{W}$ - or $\mathbf{U}$-shaped, and without or with a short stem.

\section{Description}

HEaD. Head narrower than pronotum; often produced and pointed anteriorly. Discal portion of crown glabrous with radial or longitudinal striae. Anterior margin of head angulate; texture shagreen, subcarinate, or with transverse striae. Frontoclypeus elongate; texture shagreen. Clypellus widening apically; apex following or slightly surpassing normal curve of gena. Lorum narrower, subequal to, or wider than clypellus near base. Antennal bases near middle or posteroventral (lower) corners of eyes. Antennae short, less than $1.5 \mathrm{x}$ width of head. Gena broad; not incised laterally; visible behind eyes in dorsal view; with fine erect seta beside laterofrontal suture. Antennal ledges absent. Ocelli present; close to eyes; on anterior margin of head.

THorax. Pronotum lateral margin carinate; lateral margin shorter than basal width of eye.

WINGs. Forewing macropterous; appendix restricted to anal margin; with 3 anteapical cells; veins not raised; with numerous reflexed costal veins; A1-A2 crossvein absent or present; apical venation not highly reticulate.

LEgs. Profemur with AM1 seta only; intercalary row with one row of five or more fine setae; row AV with short, stout setae or with relatively long macrosetae. Protibia dorsal surface rounded, convex. Metafemur apex macrosetae $2+2+1$. Metatarsomere I not expanded apically; plantar setae simple, tapered.

MALE GENITALIA. Valve large; parabolically shaped; articulated with pygofer and subgenital plates; lateral margin short, articulating with pygofer at a point. Pygofer dorsoapical margin not strongly incised or incised to near mid-length; basolateral membranous cleft present; macrosetae well differentiated into several rows; without processes. Subgenital plates free from each other, articulated with valve; without macrosetae or with macrosetae uniseriate laterally. Style elongate; broadly bilobed basally, median anterior lobe pronounced. Basal processes of the aedeagus/connective often present, articulated to connective or near base of aedeagus. Aedeagus with single shaft and gonopore, or rarely (Ascius) divided near apex, with two gonopores. Connective anterior arms widely divergent, broadly $\mathbf{W}$ - or U-shaped, or somewhat divergent, Y- or U-shaped; stem usually absent; articulated with aedeagus or aedeagus dissociated from connective and attached only by thin membrane. Segment X sclerotized.

Female Genitalia. Pygofer with numerous macrosetae. Ovipositor not protruding far beyond pygofer apex. First valvula convex, dorsal sculpturing pattern strigate, concatenate, reticulate, or imbricate (with overlapping scales); sculpturing reaching dorsal margin; without distinctly delimited ventroapical sculpturing. Second valvula abruptly broadened medially or subapically or broad, gradually tapered; without dorsal median tooth; teeth on apical $1 / 3$ or more; teeth small, regularly or irregularly shaped.

\section{Geography and ecology}

Distribution: Nearctic and Neotropical regions. Host plants are herbaceous and woody dicots. Scaphytopius (Cloanthanus) acutus (Say, 1830) is a vector of alfalfa witches' broom, eastern and western $\mathrm{X}$-diseases of peach, little cherry, soybean bud proliferation, clover phyllody, and western strain of North American aster yellows. S. (Convelinus) irroratus (Van Duzee, 1910) vectors the western strain of North American aster yellows. S. (Cloanthanus) magdalensis (Provancher, 1889) transmits blueberry stunt. 


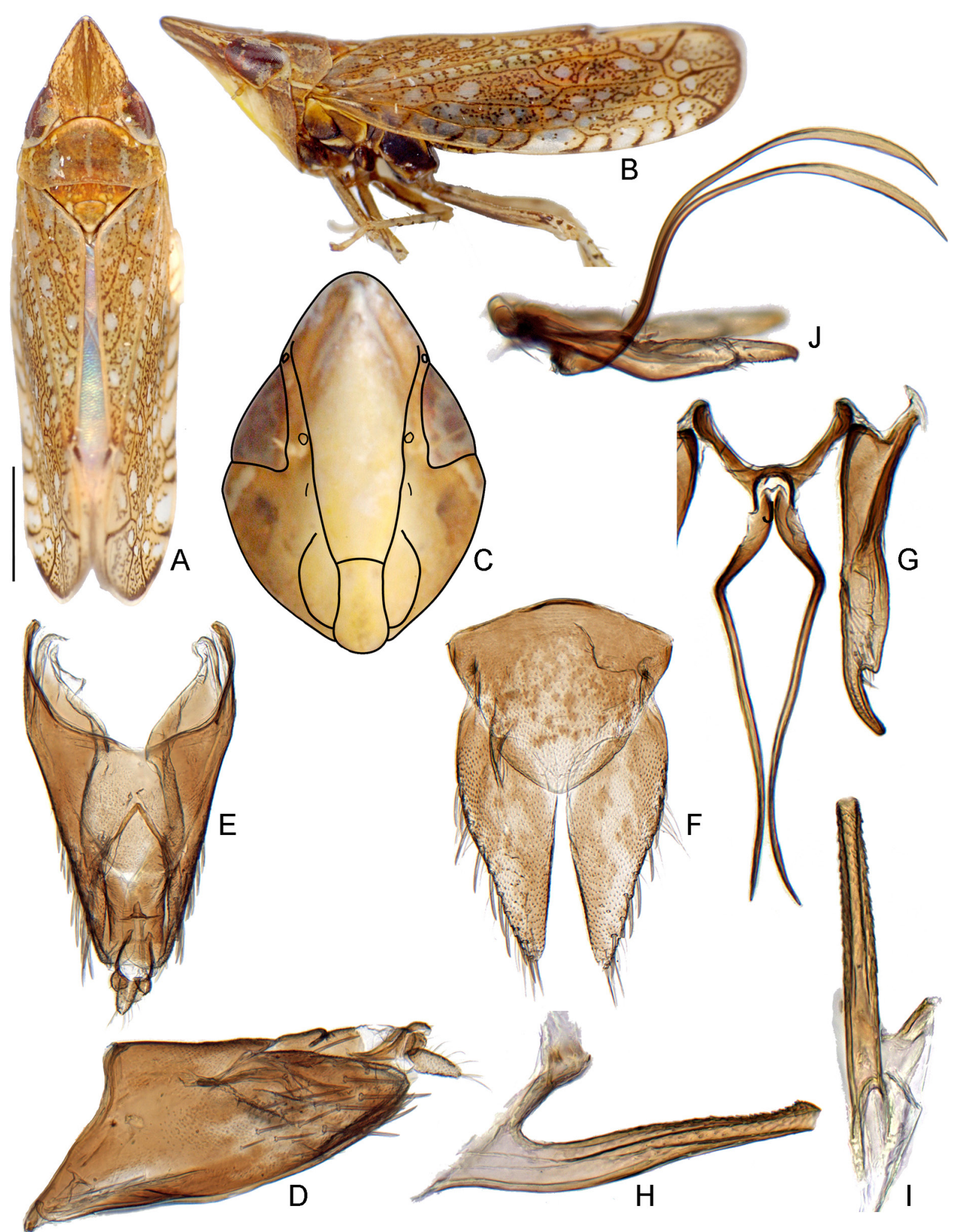

Fig. 48. Scaphytopiini. Scaphytopius acutus (Say, 1830). A-I. Standard views (see Material \& Methods). G. Connective and style shown with connective processes. J. Lateral view of connective, style and connective processes. 
S. (Convelinus) fuliginosus (Osborn, 1923) is a vector of machismo disease of legumes in Mexico and South America. S. (Convelinus) nitridus (DeLong, 1943) is a vector of western X-disease of stonefruits and celery in North America.

\section{Remarks}

Scaphytopiini contains 3 genera and 183 species. Based on phylogenetic analyses, the tribe as previously delimited (Oman et al. 1990, Webb \& Godoy 1993, Dmitriev 2002) was polyphyletic. Representatives of Japananus, Nesothamnus Linnavuori, 1959, Proceps Mulsant \& Rey, 1855, Stymphalus Stål, 1866 and 2 representatives of Scaphytopius were included in analyses and the genera were found in different areas of the tree in most analyses. There was strong support for the branch including the two Scaphytopius species, but its relationship to other Deltocephalinae was poorly resolved. In Bayesian and ML analyses, Scaphytopius was resolved within a clade including the athysanine genus Eutettix and 3 others ( $\mathrm{PP}=0.98)$. Japananus appears to be related to Opsiini, which is consistent with its bifurcate aedeagus. Ascius also has a bifurcate aedeagus, but it is retained in Scaphytopiini because it shares the numerous distinct reflexed costal veins and the widely separated anterior arms of the connective with Scaphytopius, along with the produced head and broad gena. Nesothamnus was often resolved near Idioceromimus Dietrich \& Rakitov, 2002 in our analyses. Proceps is grouped with Cicadulini with strong branch support. Stymphalus, which is placed here in the new tribe Vartini, usually grouped with an undescribed genus from Thailand (DEL 112) and some Mukariini.

The characters formerly used to define the tribe, the produced head and the gena not incised laterally and visible behind the eye in dorsal view, appear to have arisen independently multiple times in Deltocephalinae and are therefore not reliable characters on their own at the tribal level. The tribe is interpreted here to contain only three genera from the New World.

\section{Selected references}

Hepner (1947), Oman (1949), Linnavuori (1959), Webb \& Godoy (1993).

\section{Included genera}

Ascius DeLong, 1943

Scaphytopius Ball, 1931

Tenuarus DeLong, 1944

Selenocephalini Fieber, 1872

Figs 49-52

Type genus: Selenocephalus Germar, 1833.

$=$ Ianeirini Linnavuori, 1978 syn. nov.

= Adamini Linnavuori \& Al-Ne'amy, 1983 syn. nov.

= Dwightlini Linnavuori \& Al-Ne'amy, 1983 syn. nov.

\section{Diagnosis}

Selenocephalini are medium sized to large, robust, often brownish, greenish, or yellow leafhoppers, sometimes with dark irroration. They can be identified by the anterior margin of head usually with 2 or more carinae or striae, broad face, profemur row AM often with 1 or more extra setae basad of AM1, profemur row AV setae often relatively long, connective stem usually short and stout, and their distribution in the Afrotropical, Palearctic, and Oriental regions. 


\section{Description}

HEAD. Head wider than or narrower than pronotum. Discal portion of crown usually glabrous with radial or longitudinal striae, or rarely shagreen. Anterior margin of head usually acute or subfoliaceous, with 2 or more distinct carinae or transverse striae, rarely without carinae or striae. Face often quite broad. Frontoclypeus not tumid; texture shagreen. Clypellus widening apically; apex following or slightly surpassing normal curve of gena; often with more or less distinct median keel. Lorum subequal to or wider than clypellus near base. Antennal bases near middle or posteroventral (lower) corners of eyes or rarely near anterodorsal (upper) corners (Dwightlina). Antennae short, less than $1.5 \mathrm{x}$ width of head. Gena obtusely incised laterally; with fine erect seta beside laterofrontal suture, often relatively distant from laterofrontal suture. Antennal ledge somewhat developed, carinate or weakly carinate. Ocelli present; close to eyes; on anterior margin of head.

THORAx. Pronotum lateral margin carinate; lateral margin shorter than basal width of eye.

Wings. Forewing macropterous; appendix restricted to anal margin; with 3 anteapical cells; veins not raised; without reflexed costal veins; A1-A2 crossvein usually present; apical venation not reticulate or rarely highly reticulate.

Legs. Profemur usually with AM1 and with one or more additional proximal setae; intercalary row with one row of five or more fine setae; row $\mathrm{AV}$ with short stout setae or often with relatively longer setae. Protibia dorsal surface rounded, convex. Metafemur apex macrosetae $2+2+1$. Metatarsomere I not expanded apically; plantar setae simple, tapered.

Male Genitalia. Valve articulated with pygofer; lateral margin short, articulating with pygofer at a point. Pygofer basolateral membranous cleft present; macrosetae often absent or reduced, or sometimes well differentiated into several rows. Subgenital plates free from each other; articulated with valve; with macrosetae scattered, with several irregular rows near lateral margin, or without macrosetae. Style broadly bilobed basally, median anterior lobe pronounced. Basal processes of the aedeagus/connective absent or present, connected or articulated to connective or near base of aedeagus. Aedeagus with single shaft and gonopore. Connective anterior arms somewhat divergent, $\mathbf{Y}$ - or $\mathbf{U}$-shaped; stem often short, stout; articulated with or rarely fused (Abimwa) to aedeagus, or sometimes dissociated from aedeagus (Adamina).

Female genitalia. Pygofer with numerous macrosetae. Ovipositor usually not protruding far beyond pygofer apex. First valvula convex; dorsal sculpturing pattern usually strigate, sometimes concatenate or reticulate; sculpturing reaching dorsal margin; without distinctly delimited ventroapical sculpturing. Second valvula broad, tapering apically or abruptly broadened medially or subapically; often with dorsal median tooth; teeth on apical $1 / 3$ or more or restricted to apical $1 / 4$ or less; teeth small, regularly or irregularly shaped.

\section{Geography and ecology}

Distribution: Palearctic, Afrotropical and Oriental regions. Selenocephalini are usually collected on herbaceous or woody dicots and are often collected at lights. They are particularly diverse in Afrotropical forest ecosystems. Some Selenocephalini are ant-attended.

\section{Remarks}

Selenocephalini contains 21 genera and 196 species. It is interpreted here to contain all of the tribes recognized in Selenocephalinae sensu Linnavuori \& Al-Ne'amy (1983a) with the exception of Drabescini (=Paraboloponini, Bhatiini). We retain as subtribes most of the groups previously recognized as tribes of Selenocephalinae. Representatives of each of the subtribes were included in the phylogenetic analyses 
(Dwightla is included for the first time here) and the tribe was usually resolved as monophyletic, although with low branch support. The Afrotropical genus Dagama was also sometimes resolved within or as sister to this clade, and this group was resolved near Bonaspeiini. Dagama does not possess carinae on the anterior margin of the head and is not included in the tribe at this time. Further research should aim to determine the relationships between this and other Afrotropical athysanine genera and Selenocephalini, Bonaspeiini, Hypacostemmini, Phlepsiini, Scaphoideini, and Drabescini.

\section{Selected references}

Linnavuori (1978a), Linnavuori \& Al-Ne'amy (1983), Zhang \& Webb (1996), Dmitriev (2003), McKamey (2003b).

Included subtribes:

Adamina Linnavuori \& Al-Ne'amy, 1983 status nov.

Fig. 49

Type genus: Adama Dlabola, 1980.

\section{Diagnosis}

Adamina are medium sized to large yellow, green, or black leafhoppers, sometimes with longitudinal reddish stripes (subgenus Adama). They can be identified by the anterior margin of the head with 3 sharp carinae or with numerous irregular carinae, the aedeagus united to the connective only by a thin membrane, with long paraphyses (or "pseudostyles") usually articulated with or fused to the connective, and first valvulae widening apically, with irregular margins near the apex, and with reticulate sculpturing.

\section{Description}

HEAD. Head subequal to, wider than, or narrower than pronotum. Discal portion of crown glabrous with radial or longitudinal striae or shagreen. Anterior margin of head with 3 parallel carinae or with numerous irregular carinae. Frontoclypeus not tumid; texture shagreen. Clypellus widening apically. Clypellus apex following or slightly surpassing normal curve of gena. Lorum subequal to or wider than clypellus near base. Antennal bases near middle or posteroventral (lower) corners of eyes. Antennae short, less than $1.5 \mathrm{x}$ width of head. Gena obtusely incised laterally; with fine erect seta beside laterofrontal suture. Antennal ledge weakly developed (carinate or weakly carinate). Ocelli present; close to eyes; on anterior margin of head.

THorax. Pronotum lateral margin carinate; lateral margin shorter than basal width of eye.

WingS. Forewing macropterous; appendix restricted to anal margin; with 3 anteapical cells; veins not raised; without reflexed costal veins; A1-A2 crossvein present; apical venation highly reticulate or not reticulate.

LEgs. Profemur with AM1 and with one or more additional proximal setae; intercalary row with one row of five or more fine setae; row AV with relatively long macrosetae. Protibia dorsal surface rounded, convex. Metafemur apex macrosetae $2+2+1$. Metatarsomere I not expanded apically; plantar setae simple, tapered.

MALE GENITALIA. Valve articulated with pygofer; lateral margin short, articulating with pygofer at a point. Pygofer basolateral membranouscleft present; macrosetae well differentiated into several rows. Subgenital plates free from each other; articulated with valve; without macrosetae. Style broadly bilobed basally, median anterior lobe pronounced. Basal processes of the aedeagus/connective present, connected or 
articulated to connective or near base of aedeagus. Aedeagus with single shaft and gonopore; dissociated from and lying far above connective, attached to connective only by thin membrane. Connective anterior arms somewhat divergent, $\mathbf{U}$-shaped.

Female genitalia. Pygofer with numerous macrosetae. Ovipositor protruding or not protruding far beyond pygofer apex. First valvula convex; margins irregularly undulate or notched subapically; dorsal sculpturing pattern strigate, concatenate, reticulate, or with rectangular shaped cells; sculpturing reaching

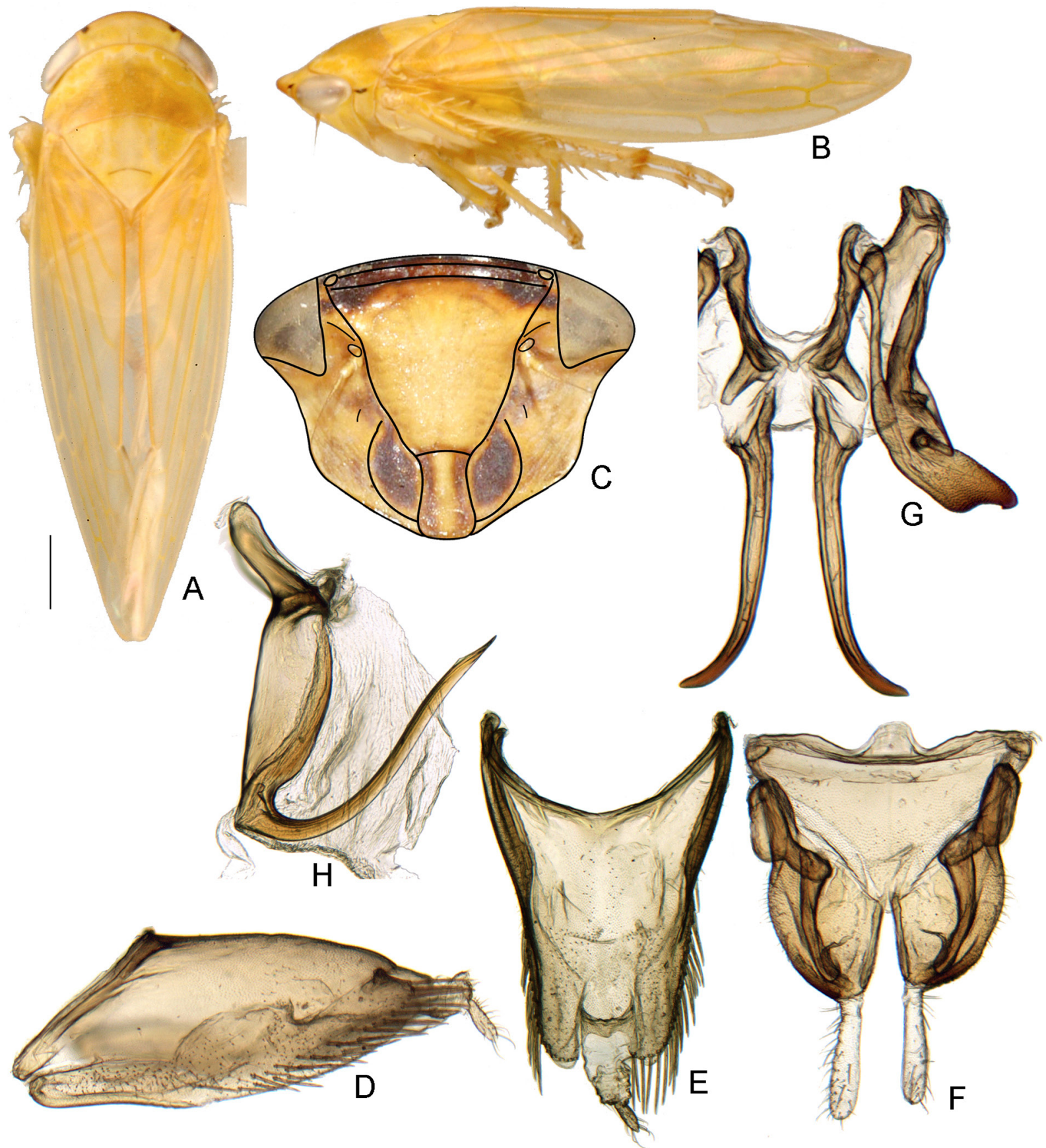

Fig. 49. Selenocephalini (Adamina). A-B, D-H. Adama (Paracostemma) amalthusia Linnavuori \& Al-Ne'amy, 1983. C. A. (Paracostemma) sp. A-H. Standard views (see Material \& Methods). G. Connective and style shown with connective processes or "pseudostyles". 
dorsal margin; without distinctly delimited ventroapical sculpturing. Second valvula abruptly broadened medially or subapically; without dorsal median tooth; teeth restricted to apical 1/4 or less; teeth small, regularly or irregularly shaped.

\section{Geography and ecology}

Distribution: Afrotropical. Adamina are frequently collected at lights and likely feed in the forest canopy.

\section{Remarks}

Adamina contains the type genus, 4 subgenera, and 49 valid species. All members are found in the Afrotropical region.

\section{Included genera}

Adama Dlabola, 1980

Dwightlina McKamey, 2003 status nov.

Fig. 50

Type genus: Dwightla McKamey, 2003.

\section{Diagnosis}

Dwightlina are large, robust, ochraceous, reddish-brown, or brown leafhoppers. They can be identified by the anterior margin of head with 3 parallel carinae, face very broad, antennal pits near upper corners of eyes, forewing and face with slight to heavy covering of fine setae, profemur with 2 or more macrosetae basad of AM1 seta, and male pygofer without macrosetae.

\section{Description}

HEAD. Head subequal to or wider than pronotum. Discal portion of crown glabrous with radial or longitudinal striae. Anterior margin of head with 3 parallel carinae. Face very broad; with slight to heavy covering of fine hairs. Frontoclypeus not tumid; texture shagreen. Clypellus widening apically; apex following or slightly surpassing normal curve of gena. Lorum subequal to or wider than clypellus near base. Antennal bases near upper or anterodorsal corners of eyes. Antennae short, less than $1.5 \mathrm{x}$ width of head. Gena obtusely incised laterally; with fine erect seta beside laterofrontal suture. Antennal ledge weakly developed (carinate or weakly carinate). Ocelli present; close to eyes; on anterior margin of head.

THORAx. Pronotum lateral margin carinate; lateral margin shorter than basal width of eye.

WINGs. Forewings macropterous; with slight to heavy covering of fine setae; tapering apically; apex thin or membranous, folded over onto opposite wing; appendix large, extending around wing apex; with 3 anteapical cells; veins not raised; without reflexed costal veins; A1-A2 crossvein present.

LEGS. Legs often with accessory setae. Profemur with AM1 and with 2 or more additional proximal setae; intercalary row with one row of five or more fine setae, sometimes with extra scattered setae; row $\mathrm{AV}$ with very long macrosetae. Protibia dorsal surface rounded, convex. Metafemur apex macrosetae $2+2+1$. Metatibia dorsal surface usually with extra stout setae, especially proximally. Metatarsomere I not expanded apically; plantar setae simple, tapered.

MALE GENITALIA. Valve articulated with pygofer; lateral margin short, articulating with pygofer at a point. Pygofer basolateral membranous cleft present; without macrosetae. Subgenital plates free from each other; articulated with valve; macrosetae irregularly arranged near lateral margin. Style broadly bilobed 


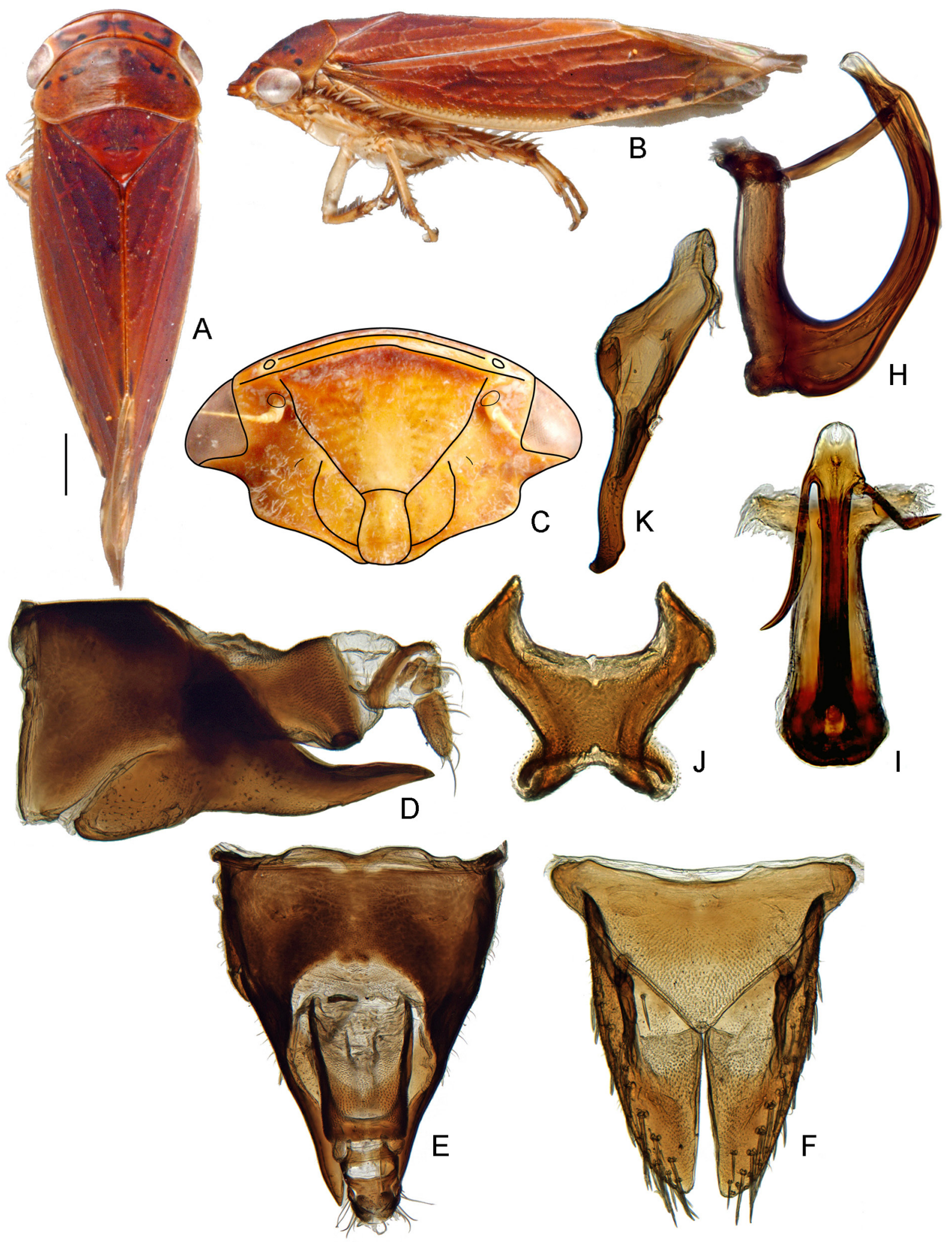

Fig. 50. Selenocephalini (Dwightlina). A-B, D-F, H-K. Dwightla delongi sp.nov. C. D. acutipennis Linnavuori \& Al-Ne'amy, 1983. A-F, H-I. Standard views (see Material \& Methods). J. Ventral view of connective. K. Ventral view of style. 
basally, median anterior lobe pronounced. Basal processes of the aedeagus/connective absent. Aedeagus with single shaft and gonopore. Connective anterior arms somewhat divergent, $\mathbf{Y}$ - or $\mathbf{U}$-shaped; stem short; articulated with aedeagus. Segment X large, sclerotized.

Female genitalia. Pygofer with numerous macrosetae. Ovipositor protruding or not protruding far beyond pygofer apex. First valvula convex; dorsal sculpturing pattern strigate; sculpturing reaching dorsal margin; without distinctly delimited ventroapical sculpturing. Second valvula slender throughout; without dorsal median tooth; dorsal teeth on apical $1 / 3$ or more or restricted to apical $1 / 4$ or less; teeth large, regularly shaped.

\section{Geography and ecology}

Distribution: Afrotropical. Dwightlina are inhabitants of forests and have been collected at lights.

\section{Remarks}

Dwightlina contains the type genus and 5 species from the Afrotropical region. McKamey (2003b) provided the replacement name Dwightla for the preoccupied Dwightia Linnavuori \& Al-Ne'amy, 1983, thus necessitating a change in the family group name. Phylogenetic analyses of new molecular data provided here confirm that Dwightla is closely related to Selenocephalini, a result also found based on morphology alone (Zahniser \& Dietrich 2008).

\section{Included genera}

Dwightla McKamey, 2003

Ianeirina Linnavuori, 1978 status nov.

Fig. 51

Type genus: Ianeira Linnavuori, 1969.

\section{Diagnosis}

Ianeirina are medium sized to large, ivory to brown leafhoppers. They can be identified by their somewhat long and slender habitus, anterior margin of the head usually with numerous carinae (shagreen and rounded to face in Ianeira), ocelli on the face below the anterior margin of the head, male pygofer without macrosetae, subgenital plates fused basally and to the valve (not fused in Bardera), and segment $\mathrm{X}$ well-sclerotized, large to very large, sometimes occupying most of the dorsal side of the pygofer.

\section{Description}

HEAD. Head subequal to, wider than, or distinctly narrower than pronotum. Discal portion of crown glabrous with radial or longitudinal striae. Anterior margin of head shagreen, with numerous transverse striations, or with numerous carinae. Frontoclypeus narrow; not tumid; texture shagreen. Clypellus widening apically, apex following or slightly surpassing normal curve of gena. Lorum subequal to or wider than clypellus near base. Antennal bases near middle or posteroventral (lower) corners of eyes. Antennae short, less than $1.5 \mathrm{x}$ width of head. Gena obtusely incised laterally; with fine erect seta beside laterofrontal suture. Antennal ledges weakly developed (carinate or weakly carinate). Ocelli present; close to or distant from eyes; on face just below anterior margin of head.

THORAX. Pronotum lateral margin carinate; lateral margin shorter than basal width of eye.

Wings. Forewing macropterous; appendix restricted to anal margin; with 3 anteapical cells; veins not raised; with or without reflexed costal veins; A1-A2 crossvein present. 


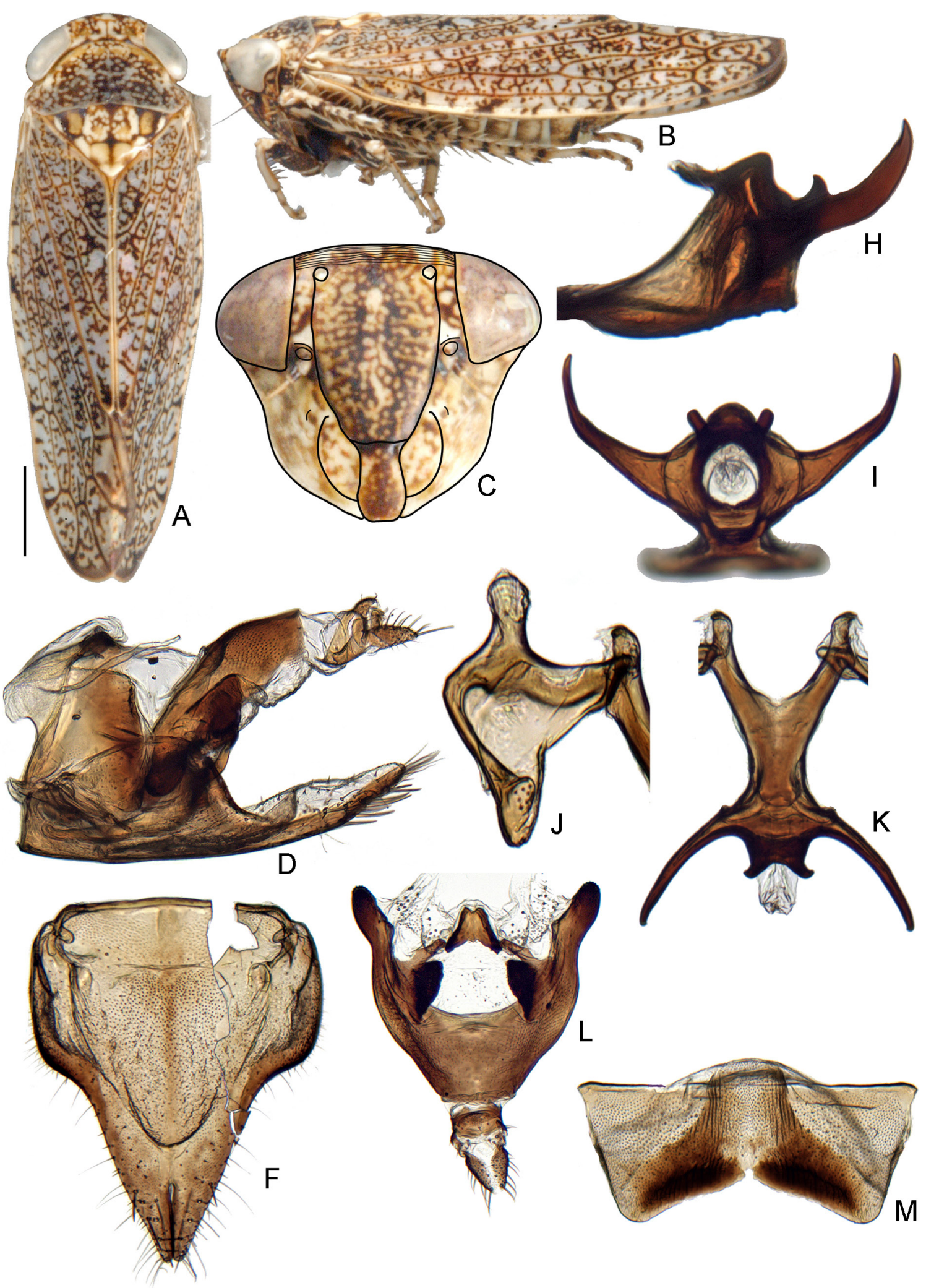

Fig. 51. Selenocephalini (Ianeirina). Abimwa knighti sp. nov. A-D, F, H-I. Standard views (see Material \& Methods). D. Pygofer shown with subgenital plates and valve. J. Ventral view of style. K. Ventral view of connective and aedeagus fused to connective. L. Dorsal view of $\partial^{\lambda}$ segments X, XI, and anus. M. $\odot$ sternite VII. 
Legs. Profemur with AM1 seta only or with AM1 and with one or more additional proximal setae; intercalary row with one row of five or more fine setae; row AV with short, stout setae. Protibia dorsal surface rounded, convex. Metafemur apex macrosetae $2+2+1$. Metatarsomere I not expanded apically; plantar setae simple, tapered.

MALE GENITALIA. Valve articulated with pygofer; lateral margin short, articulating with pygofer at a point. Pygofer basolateral membranous cleft present, macrosetae absent or reduced ( $\leq$ two rows). Subgenital plates free from each other and articulated with valve (Bardera) or fused to each other basally (apices of plates usually separated) and to valve (Abimwa, Ianeira); without macrosetae or macrosetae scattered, irregularly arranged. Style broadly bilobed basally, median anterior lobe pronounced; sometimes reduced in size (Abimwa, Ianeira). Basal processes of the aedeagus/connective absent or reduced or present, connected or articulated to connective or near base of aedeagus. Aedeagus with single shaft and gonopore. Connective anterior arms somewhat divergent, Y- or U-shaped; articulated with or fused to aedeagus. Segment X well-sclerotized; large to very large, sometimes occupying majority of dorsal side of pygofer.

Female genitalia. Pygofer with numerous macrosetae. Ovipositor not protruding far beyond pygofer apex. First valvula convex; dorsal sculpturing pattern strigate; sculpturing reaching dorsal margin, without distinctly delimited ventroapical sculpturing. Second valvula broad, gradually tapered; with dorsal median tooth; teeth on apical $1 / 3$ or more; teeth small, regularly or irregularly shaped.

\section{Geography and ecology}

Distribution: Afrotropical.

\section{Remarks}

Ianeirina contains 4 genera and 13 valid species. Males of Kanziko Linnavuori \& Al-Ne'amy, 1983 are not known. There is variability in the condition of the anterior margin of the head (rounded to face and shagreen in Ianeira, and with numerous irregular carinae in the other genera) and the condition of the male genital capsule (plates and valve articulated in Bardera and fused in Ianeira and Abimwa Linnavuori, 1978) among the genera. The monophyly of the tribe needs to be tested in a more thorough phylogenetic analysis. One representative of Ianeirini, Abimwa, was included in phylogenetic analyses and was found to be closely related to other Selenocephalini and in some analyses to Dagama Distant, 1910 (Athysanini).

\section{Included genera:}

Abimwa Linnavuori, 1978

Bardera Linnavuori \& Al-Ne'amy, 1983

Ianeira Linnavuori, 1969

Kanziko Linnavuori \& Al-Ne'amy, 1983

\section{Selenocephalina Fieber, 1872}

Fig. 52

Type genus: Selenocephalus Germar, 1833.

\section{Diagnosis}

Selenocephalina are medium sized to large, robust often brownish, yellowish, ochraceous, grey, brown, or reddish leafhoppers, sometimes with dark irroration. They can be identified by the carinae on the anterior margin of the head, ocelli on the anterior margin of the head and close to the eyes, broad face, male pygofer usually with few or no macrosetae, segment X broad and strongly sclerotized dorsally, and lacking the features defining other subtribes of Selenocephalini. 


\section{Description}

HEAD. Head subequal to or wider than pronotum. Discal portion of crown glabrous with radial or longitudinal striae. Anterior margin of head with 2 or 3 parallel carinae or with numerous irregular carinae or striations. Face very broad. Frontoclypeus not tumid; texture shagreen. Clypellus widening apically; apex following or slightly surpassing normal curve of gena; often with more or less distinct median keel. Lorum subequal to or wider than clypellus near base. Antennal bases near middle or posteroventral (lower) corners of eyes. Antennae short, less than $1.5 \mathrm{x}$ width of head. Gena obtusely incised laterally, with fine erect seta beside laterofrontal suture. Antennal ledges weakly developed (carinate or weakly carinate). Ocelli present; close to eyes; on anterior margin of head.

Thorax. Pronotum lateral margin carinate; lateral margin shorter than basal width of eye.

WINGS. Forewing macropterous; appendix restricted to anal margin or large, extending around wing apex; with 3 anteapical cells; veins not raised; without reflexed costal veins; A1-A2 crossvein present; apical venation not highly reticulate.

LeGs. Profemur with AM1 seta only or with AM1 and with one or more additional proximal setae; intercalary row with one row of five or more fine setae; row AV with short, stout setae or with relatively long macrosetae. Protibia dorsal surface rounded, convex. Metafemur apex macrosetae $2+2+1$. Metatarsomere I not expanded apically; plantar setae simple, tapered.

MaLe Genitalia. Valve articulated with pygofer; lateral margin short, articulating with pygofer at a point. Pygofer basolateral membranous cleft present; macrosetae usually absent or reduced $(\leq$ two rows). Subgenital plates free from each other; articulated with valve; without macrosetae or macrosetae scattered, irregularly arranged, uniseriate laterally, with two lateral rows of macrosetae, or with irregularly arranged macrosetae near lateral margin. Style broadly bilobed basally, median anterior lobe pronounced. Basal processes of the aedeagus/connective absent or reduced or present, connected or articulated to connective or near base of aedeagus. Aedeagus with single shaft and gonopore. Connective anterior arms somewhat divergent, Y- or U-shaped; articulated with aedeagus. Segment X broad; sclerotized dorsally at least at apex; sometimes with processes.

Female genitalia. Pygofer with numerous macrosetae. Ovipositor protruding or not protruding far beyond pygofer apex. First valvula convex; dorsal sculpturing pattern strigate; sculpturing reaching dorsal margin; without distinctly delimited ventroapical sculpturing. Second valvula abruptly broadened medially or subapically or broad, gradually tapered; often with or sometimes without dorsal median tooth; teeth on apical $1 / 3$ or more; teeth large, regularly shaped or teeth small, regularly or irregularly shaped.

\section{Geography and ecology}

Distribution: Palearctic, Afrotropical and Oriental. Selenocephalina are most diverse in the Afrotropical forested ecosystems and are often collected at lights.

\section{Remarks}

Selenocephalina contains 15 genera and 129 species. Selenocephalus was included in the phylogenetic analyses and found to be closely related to other Selenocephalini and sister to Citorus (included in phylogenetic analyses here for the first time), which is transferred from Penthimiini and included in Selenocephalina here. Citorus has a squat, robust body form, large forewing with the appendix extending around the wing apex, and protibiae flattened dorsally and carinate, which account for its previous placement in Penthimiini, but it shares other characters (ocelli on anterior margin of head and close to 


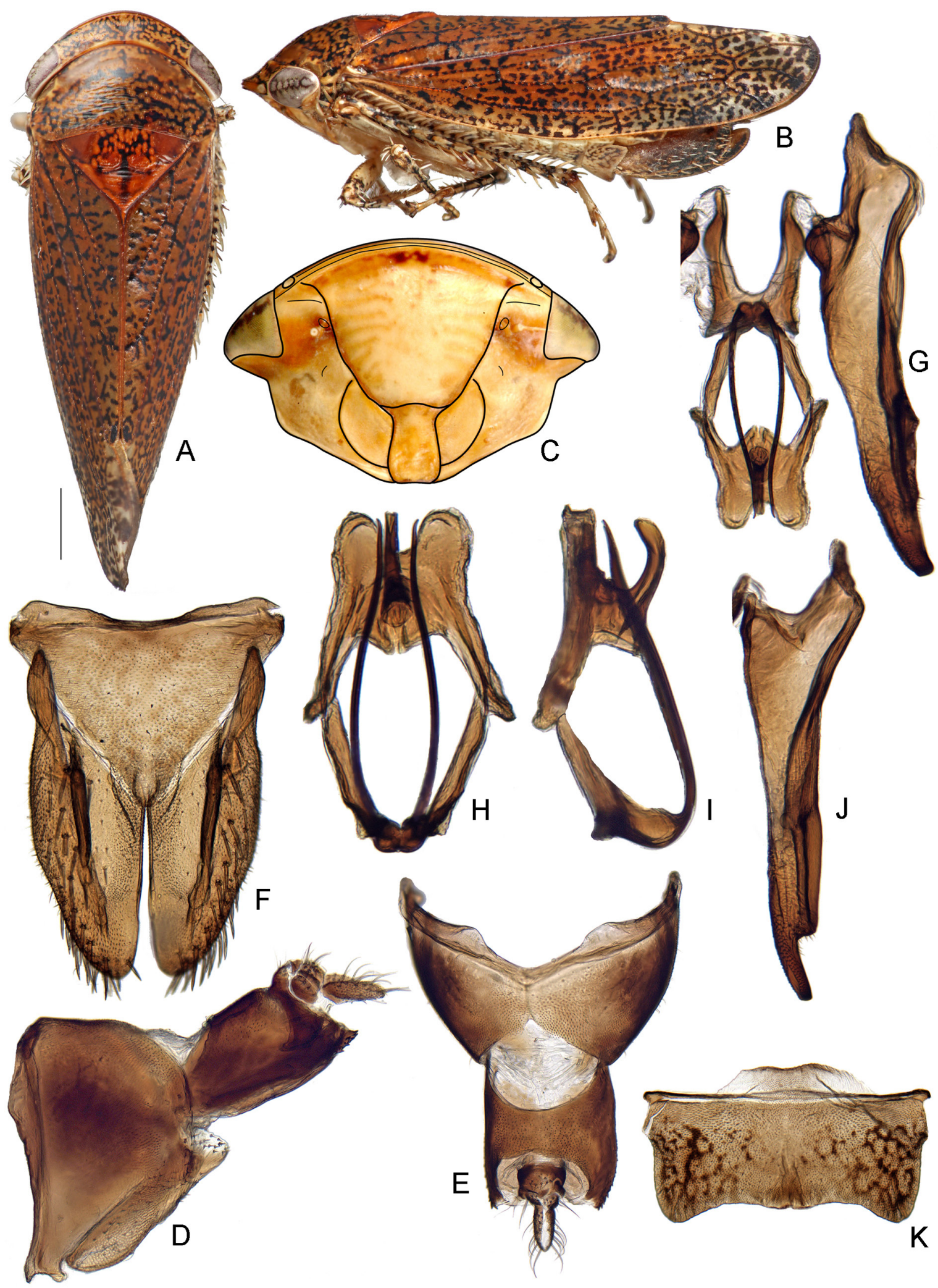

Fig. 52. Selenocephalini (Selenocephalina). A-B, D-K. Gannia viraktamathi sp. nov. C. Selenocephalus planus (Turton, 1802). A-I. Standard views (see Material \& Methods). G. Connective and style shown with aedeagus and processes of aedeagus. H-I. Aedeagus shown with processes of aedeagus. J. Broad view of style. $\mathbf{K}$. + sternite VII. 
eyes; antennal ledges not very strong) with Selenocephalina. Very strong branch support was recovered for a clade including Dwightla, Citorus and Selenocephalus.

\section{Included genera}

Angolaia Linnavuori \& Al-Ne'amy, 1983

Bumka McKamey, 2003

Citorus Stål, 1866 placement nov. (transferred from Penthimiini)

Distantia Signoret, 1879

Foso Linnavuori \& Al-Ne'amy, 1983

Gannia Theron, 1979 placement nov. (previously unplaced in Deltocephalinae)

Hecaloidella Osborn, 1934

Ifeia Linnavuori \& Al-Ne'amy, 1983

Loka Linnavuori \& Al-Ne'amy, 1983

Maichewia Linnavuori \& Al-Ne'amy, 1983

Malendea Linnavuori \& Al-Ne'amy, 1983

Moluccazhangia McKamey, 2003

Omanellinus Zhang, 1999

Sapoba Linnavuori \& Al-Ne'amy, 1983

Selenocephalus Germar, 1833

Stegelytrini Baker, 1915

Fig. 53

Type genus: Stegelytra Mulsant \& Rey, 1855.

\section{Diagnosis}

Stegelytrini are medium sized to large leafhoppers, sometimes very robust, setose, and flylike in appearance. They can be identified by the head usually much narrower than the pronotum, eyes encroaching onto the pronotum and lying above lateral carinae of pronotum, antennae usually very long, clypellus often with pair of stout subapical setae, legs with supernumerary setae, profemur row AM with AM1 plus two or more additional macrosetae, metatibia dorsal surface with extra setae between rows $\mathrm{AD}$ and $\mathrm{PD}$ near base, valve often with long length of articulation with pygofer, connective with anterior arms widely divergent, and style linear.

\section{Description}

HEAD. Head usually distinctly narrower than pronotum, rarely wider than pronotum; eyes lying above lateral carinae of pronotum. Discal portion of crown glabrous with radial or longitudinal striae. Anterior margin of head shagreen or with numerous transverse striations or carinae. Frontoclypeus not tumid, texture shagreen. Clypellus widening apically; apex following or slightly surpassing normal curve of gena; usually with pair of subapical stout setae. Lorum subequal to or wider than clypellus near base. Antennal bases near middle or posteroventral (lower) corners of eyes. Antennae usually very long, $1.5 \mathrm{x}$ width of head or longer, rarely short. Gena not incised or obtusely incised laterally; with fine erect seta beside laterofrontal suture. Antennal ledges weakly developed (carinate or weakly carinate) or strongly developed (with a definite ledge). Ocelli present; close to eyes; on anterior margin of head.

Thorax. Thorax often very large or inflated. Pronotum lateral margin carinate or not carinate; lateral margin often longer than basal width of eye, rarely shorter. Mesonotum often elongate and protuberant posteriorly. 
Wings. Forewing macropterous or submacropterous; apex sometimes truncate; appendix absent or reduced, or present and restricted to anal margin or large and extending around wing apex; with 3 anteapical cells; veins not raised; without reflexed costal veins; A1-A2 crossvein absent or present; apical venation highly reticulate or not; commissural margin usually thickened and elevated.

LEGs. Legs often with supernumerary thin and thick setae. Profemur with AM1 and with two or more additional proximal setae; intercalary row with one row of five or more fine setae, or setae scattered, not in one row; row AV with relatively long macrosetae. Protibia dorsal surface rounded, convex. Metafemur apex macrosetae $2+2+1$ or $2+2+1$ and with additional proximal macrosetae and/or with extra macrosetae clustered near apex. Metatibia dorsal surface with extra setae between rows AD and PD, especially near base; dorsal side often distinctly wider at base than at apex. Metatarsomere I not expanded apically; plantar setae simple, tapered.

MALE GENitALIA. Valve articulated with pygofer or rarely fused to pygofer; lateral margin often relatively long and with long length of articulation with pygofer, or sometimes lateral margin short, articulating with pygofer at a point. Pygofer basolateral membranous cleft often absent, rarely present; macrosetae absent or reduced ( $\leq$ two rows) or well differentiated into several rows. Subgenital plates free from each other; articulated with valve; without macrosetae or with macrosetae scattered, irregularly arranged, or uniseriate, distant from lateral margin. Style linear, median anterior lobe not pronounced. Basal processes of the aedeagus/connective absent or reduced or present, connected or articulated to connective or near base of aedeagus. Aedeagus with single shaft and gonopore. Connective anterior arms widely divergent, T-shaped or plate-like; articulated with aedeagus.

Female Genitalia. Pygofer with numerous macrosetae. Ovipositor not protruding or protruding far beyond pygofer apex. First valvula convex; dorsal sculpturing pattern strigate or concatenate; sculpturing reaching dorsal margin; without distinctly delimited ventroapical sculpturing. Second valvula abruptly broadened medially or subapically, or broad, gradually tapered; without dorsal median tooth; teeth on apical $1 / 3$ or more; teeth large, regularly shaped or teeth small, regularly or irregularly shaped.

\section{Geography and ecology}

Distribution: Palearctic and Oriental regions. Stegelytrini have been collected on trees. Recorded host families include Fagaceae and Lauraceae. Aggregations of males have been observed on wet soil.

\section{Remarks}

Stegelytrini contains 30 genera and 83 species. It is the earliest diverging lineage of Deltocephalinae, and its relictual status is reflected in the retention of primitive structures of the male genitalia such as the narrow style base and median anterior lobe of the connective. Many members of the tribe also have a suite of derived characters that are outwardly modified and produce a strange flylike or otherwise "hairy" appearance. The functional significance of these modified characters is not known, although some may be Batesian mimics of flies or bees. The tribe, which has been accorded separate subfamily status by some authors, was recently revised in a series of papers (see Selected references). We prefer to treat the group as a tribe of Deltocephalinae to accentuate the strong phylogenetic support for its close relationship to other deltocephalines.

\section{Selected references}

Webb (1999), Wei et al. (2006, 2007a, b, 2008a, b, 2010).

\section{Included genera}

Aculescutellaris Zhang, Wei \& Webb, 2007 


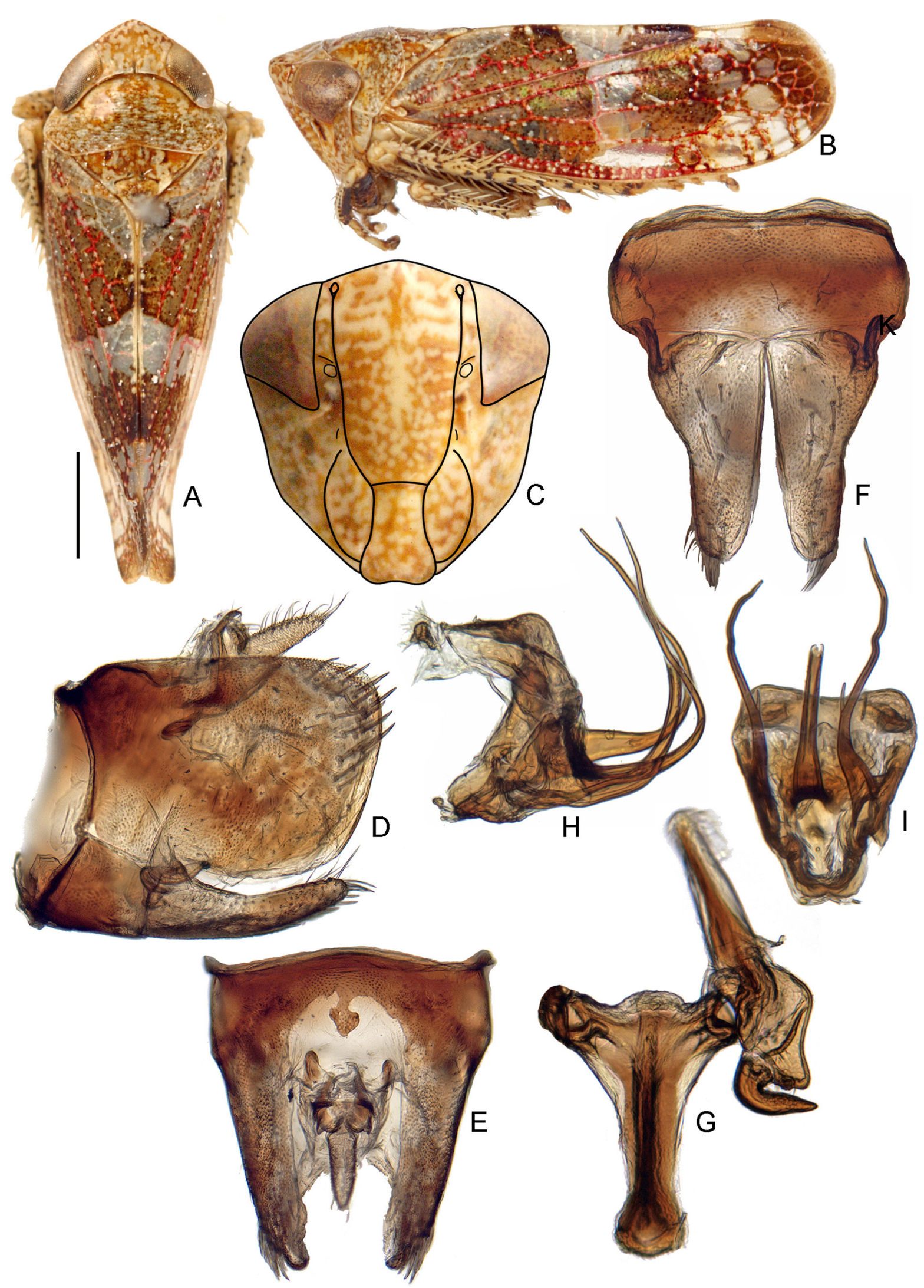

Fig. 53. Stegelytrini. Stegelytra putoni Mulsant \& Rey, 1875. A-I. Standard views (see Material \& Methods). 
Cyrta Melichar, 1902

Daochia Wei, Zhang \& Webb, 2006

Doda Distant, 1908

Honguchia Wei \& Webb, 2010

Kasinella Evans, 1971

Kunasia Distant, 1908

Louangana Wei \& Webb, 2010

Minucella Wei, Zhang \& Webb, 2008

Neophansia Wei \& Webb, 2010

Pachymetopius Matsumura, 1914

Paracyrta Wei, Webb \& Zhang, 2008

Paradoxivena Wei, Zhang \&Webb, 2006

Paraplacidellus Zhang, Wei \& Shen, 2002

Paratoba Wei, Webb \& Zhang, 2007

Pataniolidia Wei, Webb \& Zhang, 2007

Placidellus Evans, 1971

Platyvalvata Zhang, Wei \& Webb, 2006

Pseudododa Zhang, Wei \& Webb, 2007

Quiontugia Wei \& Zhang, 2010

Shangonia Wei \& Webb, 2010

Stegelytra Mulsant \& Rey, 1855

Stenolora Zhang, Wei \& Webb, 2006

Sychentia Wei \& Webb, 2010

Temburocera Webb, 1999

Toba Schmidt, 1911

Trunchinus Zhang, Webb \& Wei, 2007

Wadkufia Linnavuori, 1965

Wyuchiva Zhang, Wei \& Webb, 2006

Yaontogonia Wei \& Webb, 2010

Stenometopiini Baker, 1923

Fig. 54

Type genus: Stenometopius Matsumura, 1914 (=Hodoedocus Jacobi, 1910).

$=$ Stirellini Emeljanov, 1966.

\section{Diagnosis}

Stenometopiini are small to medium sized, often brightly colored or iridescent leafhoppers. They can be identified by the narrow crown, shagreen texture of the crown, clypellus parallel-sided or tapering apically, forewings often submacropterous to brachypterous, male pygofer sloping caudoventrally and with few macrosetae and often with a distinct lateral tooth, female ovipositor protruding far beyond the pygofer apex, first valvula dorsal sculpturing granulose to maculose and submarginal, first valvula with distinctly delimited ventroapical sculpturing, and second valvula without dorsal teeth.

\section{Description}

HEAD. Head subequal to or wider than pronotum; sometimes strongly produced or elongate. Crown narrow; discal portion of crown shagreen. Anterior margin of head shagreen, rarely sharply angulate or foliaceous if head is elongate. Frontoclypeus not tumid, texture shagreen. Clypellus parallel-sided or tapering apically, apex slightly surpassing normal curve of gena. Lorum distinctly narrower than 
clypellus near base. Antennal bases near middle or posteroventral (lower) corners of eyes. Mesial margin of eyes notched in frontal view. Antennae short, less than $1.5 \mathrm{x}$ width of head. Gena obtusely incised laterally; with fine erect seta beside laterofrontal suture. Antennal ledges absent. Ocelli present; close to eyes; on anterior margin of head.

THORAX. Pronotum lateral margin not carinate; lateral margin shorter than basal width of eye.

WINGS. Forewing macropterous to brachypterous; appendix reduced, absent, or restricted to anal margin; with 3 anteapical cells; veins not raised; usually without reflexed costal veins; A1-A2 crossvein absent; apical venation not highly reticulate.

Legs. Profemur with AM1 seta only; intercalary row with one row of five or more fine setae; row AV with short, stout setae. Protibia dorsal surface rounded, convex. Metafemur apex macrosetae 2+0, 2+1, $2+2$, or $2+2+1$. Metatarsomere I not expanded apically; plantar setae simple, tapered.

MaLe Genitalia. Valve articulated with pygofer; lateral margin short, articulating with pygofer at a point. Pygofer sloping caudoventrally; basolateral membranous cleft absent, not membranous; macrosetae absent or reduced ( $\leq$ two rows); often with distinct short tooth laterally. Subgenital plates often short, rounded; free from each other; articulated with valve; without macrosetae or macrosetae uniseriate laterally or uniseriate distant from lateral margin. Style broadly bilobed basally, median anterior lobe pronounced and usually extending anterad of lateral lobe. Basal processes of the aedeagus/connective absent or reduced. Aedeagus with single shaft and gonopore; often whip-like, long and narrow. Connective anterior arms somewhat divergent, $\mathbf{Y}$-, $\mathbf{U}$ - or narrowly $\mathbf{V}$-shaped; articulated with aedeagus.

Female Genitalia. Pygofer with macrosetae reduced or absent. Ovipositor protruding far beyond pygofer apex. First valvula not strongly convex; dorsal sculpturing pattern granulose or maculose; sculpturing submarginal; with ventroapical sculpturing distinctly delimited, long and triangular. Second valvula gradually broadened medially or subapically; without dorsal median tooth; teeth absent.

\section{Geography and ecology}

Distribution: cosmopolitan. Stenometopiini feed on grasses or sedges and often abundant in grassland ecosystems.

\section{Remarks}

Stenometopiini contains 8 genera and 96 species. Based on some morphological characters (Zahniser 2008a) and on phylogenetic analyses, the tribe appears to be most closely related to Chiasmini or to some other grass-specialist groups including Eupelicini, Drakensbergenini, and Evinus Dlabola, 1977. Most of the species are included in two genera: Doratulina, which contains only Old World species, and Stirellus, which contains both Old World and New World species. Historically, taxonomists have been inconsistent or unclear in their reasoning for their placement of species in these genera. The distinction between the two genera is therefore unclear (see Zahniser \& Nielson 2012 for further discussion), but both are retained pending further study.

Proekes Theron, 1975 and Proekoides Stiller, 1986 were previously listed in the tribe by Oman et al. (1990) but share no similarities to the tribe and are here transferred to Bonaspeiini.

\section{Selected references}

Vilbaste (1965), Emeljanov (1966, 1968), Linnavuori (1979b), Dmitriev (2004b), Zahniser (2008a), Zahniser \& Nielson (2012). 


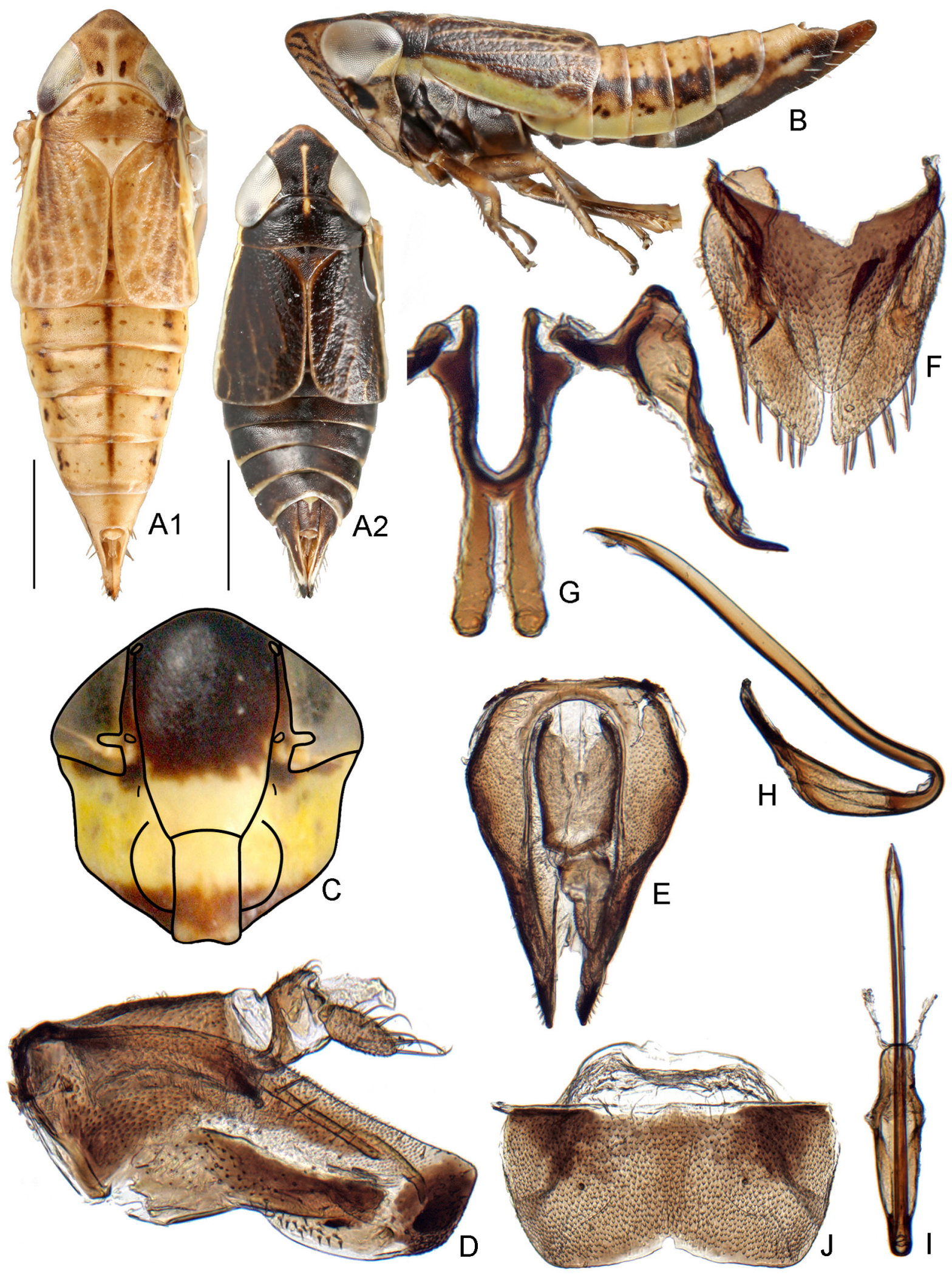

Fig. 54. Stenometopiini. A-B, D-J. Doratulina dmitrievi sp. nov. C. Stirellus bicolor (Van Duzee,

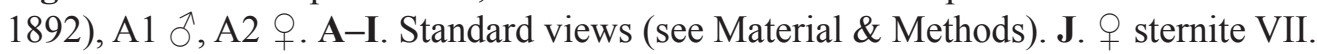




\section{Included genera}

Anaconura Emeljanov, 1999

Anemolua Kirkaldy, 1906

Doratulina Melichar, 1903

Giffardia Kirkaldy, 1906

Hodoedocus Jacobi, 1910

Kinonia Ball, 1933

Paivanana Distant, 1918

Stirellus Osborn \& Ball, 1902

Tetartostylini Wagner, 1951

Fig. 55

Type genus: Tetartostylus Wagner, 1951.

\section{Diagnosis}

Tetartostylini are medium sized, somewhat elongate, straw colored to greenish leafhoppers. They can be identified by the tapering or parallel-sided clypellus, subgenital plates fused to valve and with apices modified into sharp spines, connective with anterior arms closely appressed anteriorly, connective articulated with a pair of long processes, connective articulated with aedeagus, female sternite VII long, ovate or subovate.

\section{Description}

HEAD. Head subequal to or wider than pronotum. Discal portion of crown shagreen. Anterior margin of head shagreen. Frontoclypeus not tumid, texture shagreen. Clypellus parallel-sided or tapering apically; apex following or slightly surpassing normal curve of gena. Lorum distinctly narrower than clypellus near base. Antennal bases near middle or posteroventral (lower) corners of eyes. Antennae short, less than $1.5 \mathrm{x}$ width of head. Gena obtusely incised laterally; with fine erect seta beside laterofrontal suture. Antennal ledges absent. Ocelli present; close to eyes; on anterior margin of head.

THORAX. Pronotum lateral margin not carinate; lateral margin shorter than basal width of eye.

WINGS. Forewing macropterous; appendix restricted to anal margin; with 2 or 3 anteapical cells; veins not raised; without reflexed costal veins; A1-A2 crossvein absent; apical venation not highly reticulate.

Legs. Profemur with AM1 seta only; intercalary row reduced or absent; row AV with thin, hair-like setae or without setae. Protibia dorsal surface rounded, convex. Metafemur apex macrosetae $2+2+1$. Metatarsomere I not expanded apically; plantar setae simple, tapered.

MaLe Genitalia. Valve articulated with pygofer; lateral margin short, articulating with pygofer at a point. Pygofer basolateral membranous cleft present; macrosetae well differentiated into several rows. Subgenital plates free from each other, fused to valve; produced apically into modified spines; macrosetae uniseriate laterally. Style broadly bilobed basally; median anterior lobe pronounced. Basal processes of the aedeagus/connective present, articulated with connective. Aedeagus with single shaft and gonopore. Connective anterior arms closely appressed anteriorly, linear or loop-shaped; articulated with aedeagus.

Female genitalia. Pygofer with numerous macrosetae. Ovipositor not protruding far beyond pygofer apex. First valvula not strongly convex; dorsal sculpturing pattern imbricate (with overlapping scales); sculpturing submarginal; without distinctly delimited ventroapical sculpturing. Second valvula broad, 


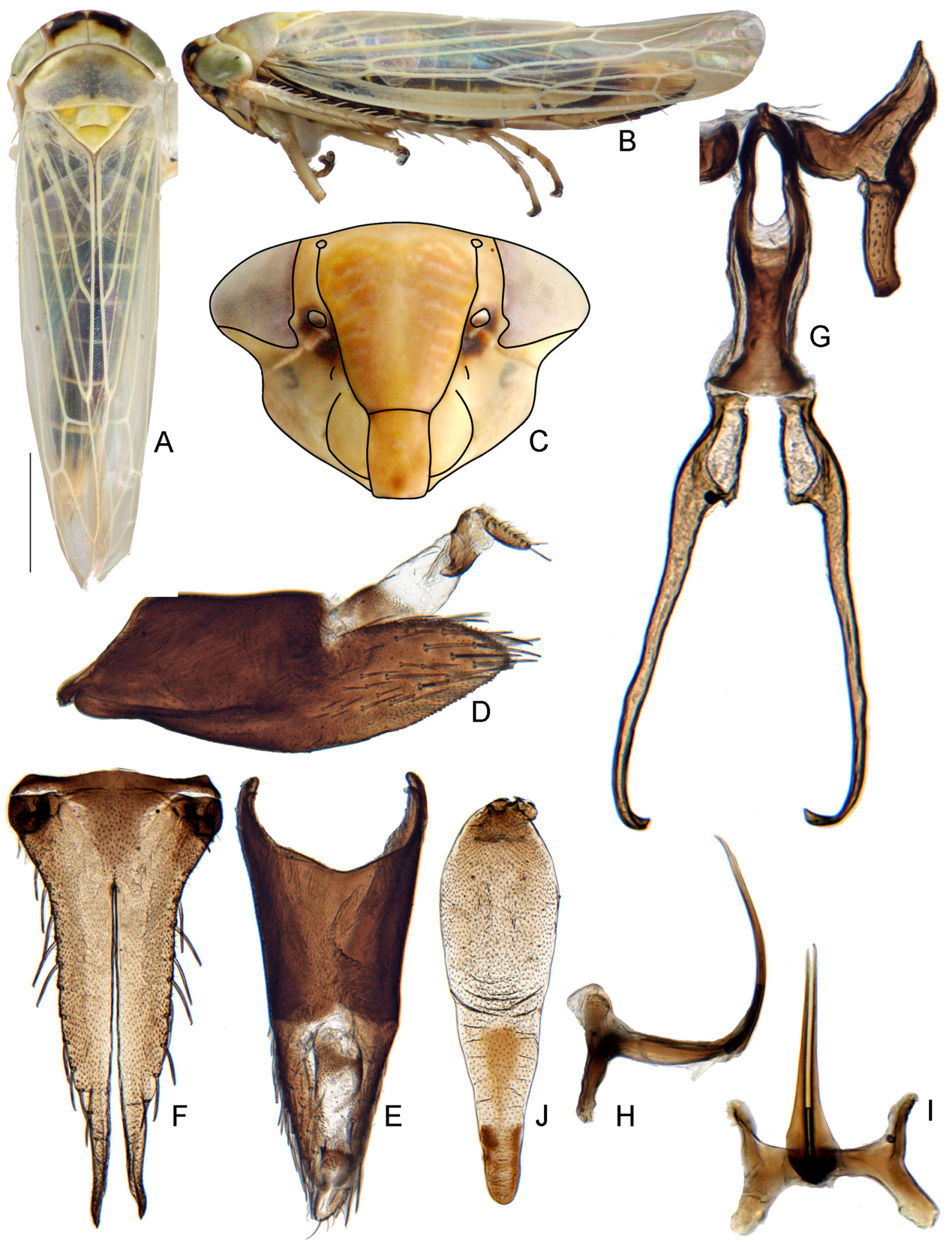

Fig. 55. Tetartostylini. A-B, D-J. Tetartostylus acutus Heller \& Linnavuori, 1968. C. Tetartostylus sp. A-I. Standard views (see Material \& Methods). G. Connective and style shown with processes of connective or "pseudostyles". J. + sternite VII. 
gradually tapered; without dorsal median tooth; teeth on apical $1 / 3$ or more; teeth small, regularly or irregularly shaped.

\section{Geography and ecology}

Distribution: Palearctic and Afrotropical regions. Tetartostylini feed on grasses.

\section{Remarks}

Tetartostylini contains the type genus and 11 species. It is resolved as sister to the Paralimnini + Deltocephalini clade in all phylogenetic analyses conducted thus far, with moderate to strong branch support. With those two tribes, it shares a tapered clypellus and a 'linear' connective. Further taxon sampling in Paralimnini could indicate that it belongs in that tribe. For now, the continued recognition of the tribe is supported by phylogenetic analyses. Dmitriev (2003) included Hiltus Theron, 1974 in the tribe based on nymphal characters, but because this genus does not have the subgenital plates fused to the valve and pseudostyli articulated with connective, we follow Theron (1974) in placing that genus in Paralimnini.

\section{Selected references}

Wagner (1951), Theron (1973), Dmitriev (2003).

\section{Notes on illustration (Fig. 55)}

The species figured in Fig. 55A-B, D-J is perhaps referable to T. acutus Heller \& Linnavuori, 1968 described from Ethiopia. A series of four males and three females (INHS) were collected from:

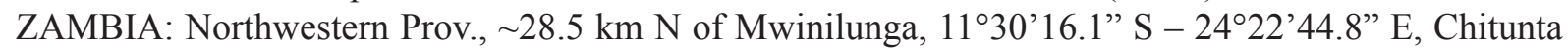
plain grassland, 1384 m, 5-7 Nov. 2007, ZA-03, sweep, JN Zahniser. This series shares with T. acutus a similar shape of the female sternite VII and similar coloration of the face. However, T. acutus was described based only on a female specimen. The females examined here are somewhat shorter (4.8$4.9 \mathrm{~mm}$ ) than that described by Heller \& Linnavuori $(5.5 \mathrm{~mm})$. For now, the Zambian specimens are tentatively identified as T. acutus, but confirmation by comparison to the type is needed. The specimens were collected in a native Zambian grassland from which numerous other undescribed species of Deltocephalinae have been identified (e.g., Doratulina dmitrievi sp. nov., below). Further exploration of the central and east African grasslands will likely reveal a rich, nearly completely undescribed fauna of Deltocephalinae.

\section{Included genera}

Tetartostylus Wagner, 1951

$$
\begin{aligned}
& \text { Vartini tribe nov. } \\
& \text { urn:Isid:zoobank.org:act:E1F01DC6-889F-48C3-B0D7-28C87B614F1D }
\end{aligned}
$$

Fig. 56

Type genus: Varta Distant, 1908.

\section{Diagnosis}

Vartini are medium sized to large, somewhat elongate, usually greenish or bluish leafhoppers, usually with red or orange longitudinal stripes. They can be identified by the produced and pointed head, gena visible behind the eye in dorsal view, elongate frontoclypeus, lorum distant from genal margin, profemur intercalary row setae thick and extending to or beyond middle of profemur, forewings truncate apically, apodemes of male sternite II long, subrectangular, flared apically, and pointed posterolaterally, 
connective with anterior arms appressed, and male segment $\mathrm{X}$ tube-like and protruding from pygofer and often well-sclerotized.

\section{Description}

HEAD. Head narrower than pronotum; acutely or bluntly produced. Discal portion of crown glabrous with radial or longitudinal striae. Anterior margin of head foliaceous or acutely angled with several transverse striae or carinae. Frontoclypeus very long, narrow; texture shagreen; sometimes with median longitudinal carina anterodorsally. Clypellus parallel-sided or widening apically; apex following or slightly surpassing normal curve of gena. Lorum distinctly narrower than clypellus near base; very wellseparated from genal margin. Antennal bases near middle, posteroventral (lower) corners, or anterodorsal (upper) corners of eyes. Antennae long, $1.5 \mathrm{x}$ width of head or more. Gena not incised, slightly incised, or sinuate laterally; visible behind eye in dorsal view; with fine erect seta beside laterofrontal suture. Antennal ledges absent. Ocelli present; close to eyes; on anterior margin of head.

THORax. Pronotum lateral margin carinate; lateral margin shorter than basal width of eye.

WINGS. Forewing macropterous; truncate apically; appendix absent, reduced, or present and restricted to anal margin; with 3 anteapical cells; veins sometimes obscure; veins not raised; without reflexed costal veins; A1-A2 crossvein absent; apical venation not highly reticulate.

LEGS. Setal bases often darkly colored. Profemur with AM1 seta only; intercalary row setae relatively large and thick, extending to or past middle of profemur; row AV with short, stout setae. Protibia dorsal surface rounded, convex; row AD with 1 apical macrosetae; row PD with 5 to 9 macrosetae. Metafemur apex macrosetae $2+2+1$. Metatarsomere I not expanded apically; plantar setae simple, tapered.

MaLe genitalia. Apodemes of sternite II long, subrectangular, flared apically, pointed posterolaterally. Valve articulated with pygofer; lateral margin short, articulating with pygofer at a point. Pygofer basolateral membranous cleft present or absent; macrosetae absent or reduced or present, well differentiated into several rows. Subgenital plates free from each other or fused or partially fused to each other or to valve; often with many scattered macrosetae, or with one to several rows of macrosetae near lateral margin. Style broadly bilobed basally; median anterior lobe pronounced. Basal processes of the aedeagus/connective absent or present, if present then fused to connective or aedeagus. Aedeagus with single shaft and gonopore. Connective anterior arms closely appressed anteriorly, or rarely slightly separated; stem absent or present; articulated with or fused to aedeagus. Segment X tube-like; protruding from pygofer; sometimes with ventral processes.

Female genitalia. Pygofer with numerous macrosetae. Ovipositor protruding or not protruding far beyond pygofer apex. First valvula convex; dorsal sculpturing pattern strigate to reticulate; sculpturing reaching dorsal margin; without distinctly delimited ventroapical sculpturing. Second valvula broad, gradually tapered or slightly expanded subapically; without dorsal median tooth; teeth on apical 1/4 to $1 / 3$ or more; teeth small to medium sized, regularly or irregularly shaped.

\section{Geography and ecology}

Distribution: Afrotropical, Palearctic, and Oriental regions. Linnavuori (1978b) reported Stymphalus Stål, 1866 "on sedges and grasses in moist locations".

\section{Remarks}

Vartini includes 7 genera and 23 species. The tribe is newly described here to include seven genera previously placed in Scaphytopiini. The only diagnostic characters that Vartini share with Scaphytopius are the produced head and very broad gena visible behind the eyes in dorsal view. These two characters 

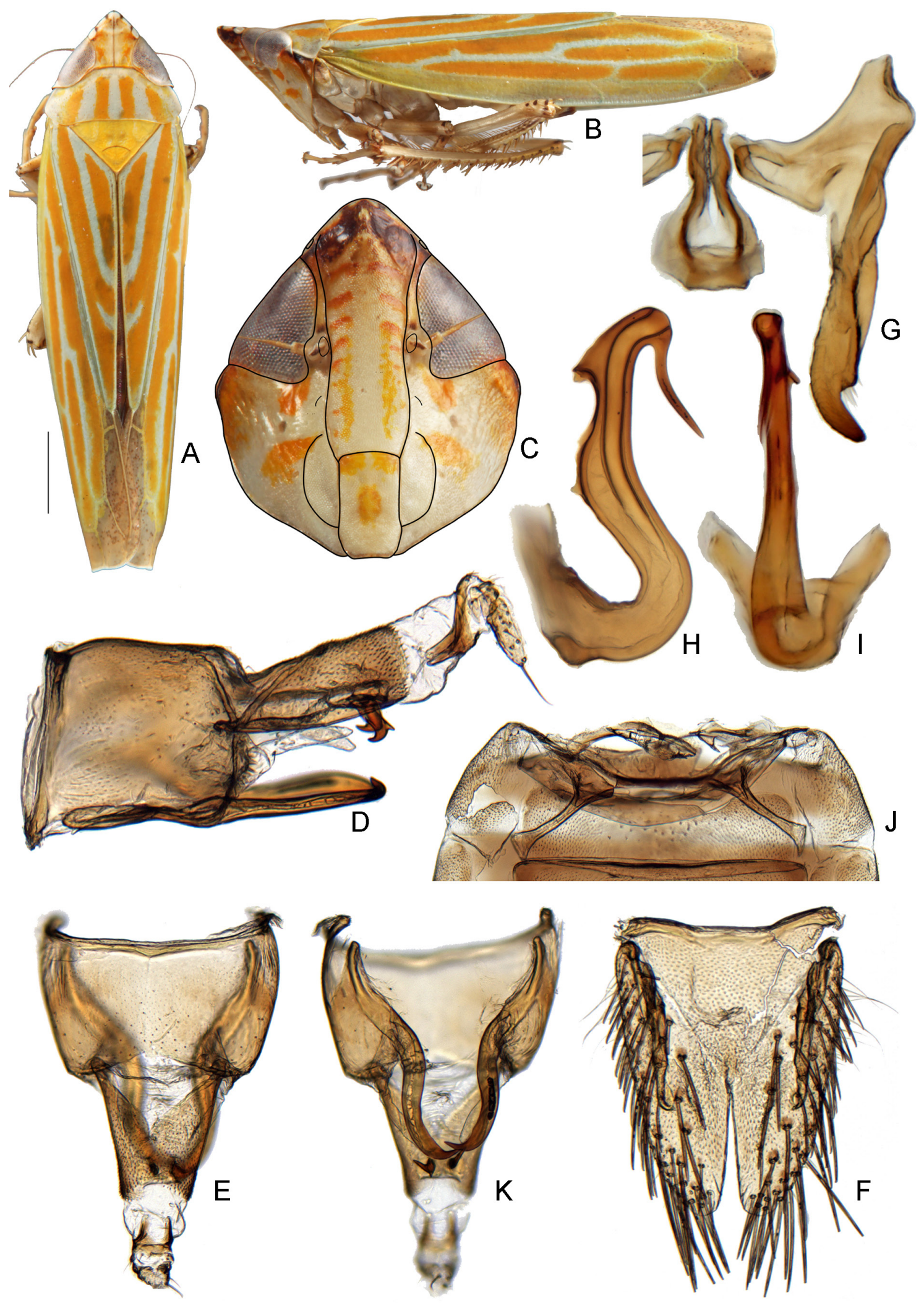

Fig. 56. Vartini. A-C. Vartalapa robusta Viraktamath, 2004. D-K. Varta rubrofasciata Distant, 1908. A-I. Standard views (see Material \& Methods). J. Ventral view of base of $\widehat{\partial}$ abdomen showing long apodemes of sternite II (sternite I obscure). K. Ventral view of $\lesssim$ pygofer. 
tend to co-occur in many deltocephalines that are not related to one another. None of the genera previously included in Scaphytopiini that were sampled in phylogenetic analyses here (Scaphytopius, Nesothamnus, Japananus, Stymphalus) were found to be closely related to one another. Thus, the aforementioned characters appear to be convergent and not appropriate for sole use in defining tribes of Deltocephalinae. The Vartini are separated from Scaphytopiini here and, based on the number and uniqueness of the characters defining the group, appear to form a monophyletic group. Three characters supporting the group are newly reported here: profemur intercalary row setae relatively thick and extending to or basad of midlength of femur; apodemes of male sternite II long, subrectangular, flared apically, and pointed posterolaterally; male segment $\mathrm{X}$ tube-like and protruding from pygofer, often well-sclerotized. Viraktamath (2004) revised the group and several new species have been described since.

Stymphalus was resolved near some Mukariini (Mukaria and Scaphotettix) and sister to an undescribed genus from Thailand which does not share the diagnostic characters of the tribe listed above. More data are needed to resolve its relationship to other tribes of Deltocephalinae.

\section{Selected references}

Linnavuori (1978b), Viraktamath \& Anantha Murthy (1999), Viraktamath (2004).

\section{Included genera:}

Curvimonus Viraktamath \& Anantha Murthy, 1999 placement nov. (transferred from Scaphytopiini) Shivania Viraktamath, 2004 placement nov. (transferred from Scaphytopiini) Stymphalus Stål, 1866 placement nov. (transferred from Scaphytopiini) Varta Distant, 1908 placement nov. (transferred from Scaphytopiini) Vartalapa Viraktamath, 2004 placement nov. (transferred from Scaphytopiini) Vartatopa Viraktamath, 2004 placement nov. (transferred from Scaphytopiini) Xenovarta Viraktamath, 2004 placement nov. (transferred from Scaphytopiini)

\section{New species descriptions}

\section{Acostemmini}

Acostemma stilleri sp. nov. urn:1sid:zoobank.org:act:202D6A00-1E0B-4990-A9CF-81B700031C68 Fig. 9

\section{Diagnosis}

There are 11 valid species of Acostemma, 8 of which are known from Madagascar. The male genitalia of the new species appear to be unique and do not match any of the 6 species for which genitalia were illustrated by Evans (1954) or A. phylia Evans, 1959. The genitalia of A. suboecia (Jacobi) were not illustrated, but the size of the male is reported to be $12.5 \mathrm{~mm}$, much larger than the species described here. Of the species illustrated by Evans (1954), A. stilleri may be most similar to A. sulcifrons (Signoret), but the processes arising from the preatrium are much longer in $A$. stilleri and the the processes arising from the shaft of the aedeagus are differently shaped.

\section{Etymology}

This species is named in honor of M. Stiller, the leading authority on South African Deltocephalinae. 


\section{Type material}

\section{Holotype}

§., MADAGASCAR, Toliara Prov., Parc Nat. de Tsimanampetsotsa, Forêt de Bemanateza, $20.7 \mathrm{~km} 81^{\circ}$

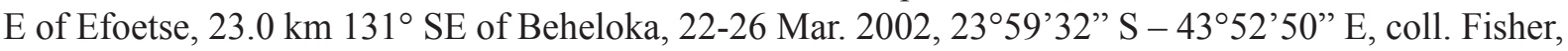
Griswold et al., California Academy of Sciences, at light - in spiny forest thicket, elevation $90 \mathrm{~m}$, code: BLF6253. Deposited in coll. CAS.

\section{Paratypes}

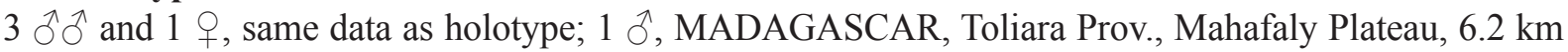
$74^{\circ}$ ENE of Itampolo, elev. 80 m, 21-25 Feb. 2002, 24³9'13" S - 4359'48” E, coll. Fisher, Griswold et al., Calif. Acad. of Sciences, malaise trap - spiny forest thicket, code: BLF5759; $4 \hat{\delta} \widehat{\delta}$ and 3 우 MADAGASCAR, Toliaran Prov., Parc National d'Andohahela, Forêt de Manantalinjo, $33.6 \mathrm{~km} 63^{\circ}$

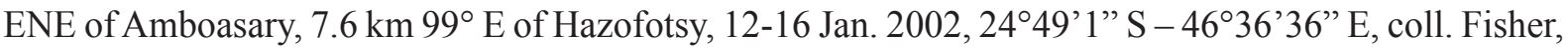
Griswold et al., California Academy of Sciences, at light - in spiny forest/thicket, elevation $150 \mathrm{~m}$, code: BLF4813.

One of the $\delta$ paratypes from locality BLF6253 is completely cleared and stored in glycerine. Genomic DNA was extracted from this specimen, and DNA sequence data generated from it are included in the analyses here. The specimen bears the voucher number "DEL 014". 5 paratype males and 3 females deposited at CAS, 3 paratype males and 1 female deposited at INHS.

\section{Description}

Size. ô 8.5-9.5 mm, o 8-10 mm.

Body. Head as wide as pronotum. Crown slightly produced medially; longer medially than next to eyes; surface texture longitudinally striate; depressed with distinct transition from vertex to frons; upturned at apex. Anterior margin of head with single carina between ocelli. Face with epistomal suture evident just below and mesad of ocelli; face shagreen below epistomal suture, rugose or irregular above. Clypellus long, widening apically. Gena broad. Antennal ledge absent. Forewing wing with several extra crossveins in anteapical and/or apical cells. A1-A2 crossvein present. Profemur row AM with AM1 and 2 or more additional proximal macrosetae; intercalary row with apical setae long, fine and with proximal setae long, thick, extending more than half length of femur; row AV with short, stout setae. Protibia dorsal surface rounded; macrosetae 1+5. Mesofemur row AV with numerous short, stout setae. Metafemur apical setae $2+2+1$. Metatarsomere I pecten with 6 long platellae, without tapered setae.

MALE. Pygofer broad, rounded; with numerous short, thick setae posteriorly and posteroventrally; fused to valve. Subgenital plates short; fused to each other; with several macrosetae subapically. Style in lateral view bent apically at nearly $90^{\circ}$ angle; apophysis curved, somewhat thick. Connective with anterior arms separate at slightly more than $90^{\circ}$; median stem absent; with short posterolateral pair of arms; fused with aedeagus. Aedeagus lying on top of and fused to connective; preatrium strongly modified into recurved process, bifurcated near connective forming pair of slender acuminate processes; aedeagal shaft with two asymmetrical processes, one arising from base parallel to shaft and terminating near gonopore, the other arising near apex, curved anteriorly, extending beyond gonopore; gonopore apical. Segment X long, narrow; $2.25 \mathrm{x}$ longer than wide.

Female. Sternite VII length $\sim 1.75 \mathrm{x}$ width; pointed and $\mathrm{V}$-shaped apically; lateral margins convex anteriorly, concave posteriorly. First and second valvulae illustrated in Zahniser \& Dietrich (2008: figs 5b, 7d) as "Acostemma sp.". First valvulae relatively slender; dorsal sculpturing pattern strigate to reticulate or imbricate. Second valvulae slender; with $\sim 6$ knob-like dorsal teeth restricted to less than apical 1/4 of valvulae. The eggs of this species are very unusual in having a tuft of lamellate seta-like extensions 
at one end. This has not been observed in any other Deltocephalinae, but a similar modification was reported by Wei et al. (2007b) in the stegelytrine Patanolidia magnifica Wei \& Webb. The setae appear to be more filamentous in that species, and occupy a greater portion of the egg than in A. stilleri. Eggs of numerous species of Deltocephalinae have been observed and are usually unremarkable in structure.

\section{Remarks}

At least 20 other species of Acostemma were collected in the Terrestrial Arthropod Inventory of Madagascar project conducted by the California Academy of Sciences (CAS) and Tsimbazaza Botanical and Zoological Park (PBZT), and none were assignable to described species. The available illustrations of the genitalia of Acostemma spp. are not very detailed, and a revision of the genus would add much needed clarity to the taxonomy of the currently known species.

\section{Arrugadini}

Arrugada linnavuorii sp. nov. urn:Isid:zoobank.org:act:24E57486-2093-4068-8054-57D22C2F59BD

Fig. 10 A-B, D-J

\section{Diagnosis}

A. linnavuorii is similar to A. rugosa Osborn, 1924 and A. affinis (Osborn, 1924) in the shape of the pygofer but can be distinguished from them by the medially unproduced head (produced in A. rugosa and $A$. affinis) and the shape of the aedeagus which, is longer, more bowed, and apex not spinose in the new species.

\section{Etymology}

This species is named in honor of Dr. Rauno E. Linnavuori, author of 5 valid tribes, 2 subtribes, 127 genera and 671 species of Deltocephalinae.

\section{Type material}

\section{Holotype}

§̂, PERU, Pasco, Villa Rica, 100 m, 23 Nov. 1971, R.T. \& J.C. Schuh, coll. AMNH.

\section{Paratypes}

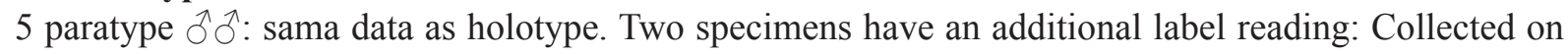
mud road in forested area just east of town. Two other specimens have pink labels, one with \#2885 and the other with $\# 2990.2$ paratypes are at AMNH and the remaining paratypes are at INHS.

\section{Description}

SizE. $\widehat{\overbrace{}} 7.5-8.0 \mathrm{~mm}$.

Body. Head wider than pronotum. Crown dark brown to black in color, sometimes yellowish in part; not produced medially; median length equal to length next to eye; texture strongly striate to rugose; depressed; sharply angled to face. Ocelli on anterior margin of head; 2-3x their diameter from adjacent eyes. Face strongly rugose. Clypellus widening apically. Antennal ledges distinct. Pronotum yellow anteriorly, dark brown to black on posterior $2 / 3$; carinate laterally; lateral carina longer than half basal width of eye. Mesonotum yellow with pair of dark spots medially. Forewing brown; claval veins yellow; sometimes with a few extra crossveins; usually with 1 to several crossveins between A1 and claval suture. Protrochanter with stout apical seta. Profemur row AM with AM1 only; intercalary row with long fine setae; row AV with $\sim 4$ to 7 relatively long thick setae. Protibia flattened dorsally; dorsal margins 
angulate; macrosetae 5+1. Mesotrochanter with stout apical PV seta. Mesofemur row AV with $\sim 4$ to 6 relatively long thick setae. Metafemur apical setae $2+1+1$. Metatarsomere I plantar setae irregularly arranged.

MALE. Pygofer incised dorsally to midlength; with several rows of macrosetae on posterior half; hind margin notched posterodorsally; lobate ventrally; on either side of basolateral cleft, pygofer lobes overlap, with posterior lobe articulating with dorsal extension of subgenital plate. Subgenital plates constricted medially; macrosetae uniseriate distant from lateral margin. Connective Y-shaped; stem as long as or longer than anterior arms; articulate with aedeagus. Style broadly bilobed basally; apophysis short, digitate. Aedeagus with base of shaft tall, turning dorsad after some length; tapering apically; gonopore subapical on ventral side. Segment X strongly sclerotized dorsally; elongate, narrow; basally with protuberant dorsal lobe.

\section{Remarks}

One specimen is especially lightly pigmented but has identical male genitalia. Also collected at the same time were three specimens of A. rugosa. These individuals, including the new species, were apparently mud-puddling, which has been reported before in A. affinis by Rakitov et al. (2005).

\section{Drabescini}

Drabescus zhangi sp. nov. urn:1sid:zoobank.org:act:899B96B1-7890-4256-8AF9-599E63134DB2

Fig. 19

\section{Diagnosis}

Externally, the new species is very similar in coloration to D. natalensis Distant, 1910, the only other known African species of Drabescus. It can be distinguished from that species by the following characters: crown not as strongly produced, coloration of crown not as mottled, style apophysis spatulate (digitate in natalensis), aedeagus with pair of long processes arising from ventral margin (natalensis with pair of short apical processes), and segment $X$ with pair of long filamentous setae arising from ventral margin (absent in natalensis). The holotype was compared with specimens of $D$. natalensis which were collected at the same locality.

\section{Etymology}

This species is named in honor of Dr. Yalin Zhang, who has contributed extensively to knowledge of the Oriental leafhopper fauna.

\section{Type material}

\section{Holotype}

đ, CENTRAL AFRICAN REPUBLIC, Maboke, 6 May 1969, Michel Boulard, coll. AMNH.

\section{Description}

SIZE. ơ $9.2 \mathrm{~mm}$.

BoDy. Head wider than pronotum. Crown very slightly produced medially; surface shiny with longitudinal and radial striations; dark brown medially at posterior margin, with transverse ivory band reaching posterior margin laterally, with brown band anteriorly; with pair of dark brown spots near eyes; sharply angled to face. Anterior margin of head with numerous irregular carinae; with distinct transverse ivory band leading onto eyes. Frontoclypeus sharply keeled below dorsal margin; keel confluent laterally with 
distinct oblique antennal ledges; texture longitudinally striate; dark brown. Clypellus constricted near base; expanding apically; dark brown. Lorum dark brown; wider than clypellus at base. Gena ivory below eye, dark brown above. Antennal sockets near upper corners of eyes. Antennae long, $1.5 \mathrm{x}$ width of head or longer. Pronotum and mesonotum ivory with 5 broad longitudinal brown or black stripes; median 3 stripes tapering anteriorly and posteriorly. Pronotum carinate laterally. Forewings smoky; veins brownish with very small whitish callosities, each bearing a very small seta; without extra crossveins; with crossvein between A1 and claval suture; with A1-A2 crossvein. Profemur row AM without distinct setae; intercalary row with numerous scattered fine setae, not in a distinct row; row AV with only scattered fine setae. Protibia dorsal surface flat; dorsal margins carinate. Mesofemur with scattered longer ventral setae. Mesotibia dorsal surface flattened, margins carinate. Metafemur apical setae 2+1. Metatibia row PD setae uniform in length.

MALE. Pygofer incised dorsally past midlength; laterally with several macrosetae subapically; each side with spine arising from ventral margin, slightly sinuate, directed posteriorly, surpassing pygofer sides. Subgenital plates long, triangular; lateral margins straight to slightly concave; without macrosetae; with several short fine hairs laterally. Connective Y-shaped; stem about as long as anterior arms; articulate with aedeagus. Style broadly bilobed basally; preapical lobe squarish; apophysis incrassate, spatulate, with stippled texture. Aedeagus $\mathbf{U}$-shaped; with pair of slender process arising from shaft near ventral margin, following shaft, slightly surpassing apex of shaft; gonopore apical. Segment X broad; sclerotized laterally and dorsally on posterior half; with pair of very long filamentous setae arising from ventral side, extending beyond pygofer.

\section{Parabolopona webbi sp. nov. urn:lsid:zoobank.org:act:08E017C2-9224-4514-9F1F-CA86CCBFAA6A}

Fig. 20

\section{Diagnosis}

P. webbi is similar to P. luzonensis Webb, 1981 with which it shares the preapically and sharply bent style apophysis. It can be distinguished from that species by shorter, wider stem of the connective, the aedeagus with a single basal process ( $P$. luzonensis with a pair of basal processes), and apex of aedeagus with a pair of processes ( $P$. luzonensis without processes). It is also similar to the recently described $P$. mutabilis Ohara \& Kogure, 2012, with which it shares the unpaired ventral process of the aedeagus. Ohara \& Kogure (2012) report significant variation in the male genitalia of $P$. mutabilis, but $P$. webbi appears to fall outside of the range of variability described and can be distinguished by the pygofer more deeply excavated caudodorsally, the laterally directed apical processes of the aedeagus, the aedeagal shaft narrower in lateral view, the ventral process of the aedeagus more widely separated from shaft, and style apophysis longer and more sharply bent.

\section{Etymology}

The new species is named in honor of Michael D. Webb of the Natural History Museum, London, a leading world authority on leafhoppers.

\section{Type material}

Holotype

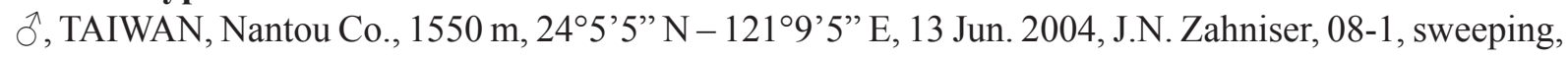
coll. NMNS.

\section{Description}

Size. $\widehat{\text { Oे }} 7.0 \mathrm{~mm}$. 
CoLOR. General color yellowish to greenish with two light orange longitudinal stripes from anterior margin of head to mesonotum.

BoDy. Head about as wide as pronotum; produced; about $2 \mathrm{x}$ longer medially than next to eye. Crown texture shagreen with slight longitudinal or radial striations; transversely furrowed medially. Ocelli on anterior margin of head, slightly dorsad; 3-4 x their own diameter from adjacent eye. Anterior margin of head sharply angled; with two sharp carinae and numerous smaller irregular carinae. Frontoclypeus flat; shagreen; depressed near anterior margin; relatively long. Clypellus expanding apically. Lorum wider than clypellus at base. Antennal sockets near upper corners of eyes; antennal pits very large, encroaching onto frontoclypeus; antennae very long, about 0.5 length of body. Pronotum carinate laterally; lateral margin nearly half as long as eye width at base. Forewing yellowish-greenish; smoky and more hyaline apically; apical veins darkly pigmented at apex; commissural margin with 3 small dark spots, 1 each where anal veins meet margin, 1 at apex; with small dark spot at $\mathrm{Cu}-\mathrm{M} 1$ and at base of $\mathrm{M} 3+4$. Legs lightly colored and with numerous dark pigment spots, especially at bases of setae. Profemur row AM with AM1 seta only; intercalary row with 11-13 fine setae; row AV reduced to very small fine hairs. Protibia rounded dorsally; macrosetae 1+4. Mesotrochanter with stout apical PV seta. Mesofemur rows AV and PV each with 3 distinct long macrosetae. Mesotibia dorsal macrosetae 4+4. Metafemur apical setae $2+2+1$. Metatibia row PD setae relatively long, longer setae nearly twice length of row AD macrosetae; row PD setae alternating shorter and longer. Metatarsomere I pecten setae with 3 platellae between tapered AV and PV setae.

MALE. Pygofer sclerotized dorsally nearly to mid-length; sides somewhat produced posteroventrally; with numerous setae posteroventrally; inner wall with blade-like process arising from posterodorsal margin, directed ventrally. Subgenital plates broad, rhomboidal in shape; apices digitate; without macrosetae. Connective Y-shaped; anterior arms long, slender; stem fused to or modified into single lanceolate process, process with longitudinal striate or rough sculpturing apically. Style broadly bilobed basally; preapical lobe sharply angulate; apophysis sharply pointed medially; apex sharp, directed laterally; with some stippled sculpturing preapically. Aedeagus with socle large; base broad; with unpaired medial lanceolate process arising ventrally near base; shaft nearly straight, slightly upturned apically; apex with pair of short apical processes directed dorsally then bent laterally; gonopore subapical on ventral side. Segment X sclerotized laterally and somewhat dorsally; longer than wide.

\section{Remarks}

The holotype specimen is slightly teneral, but the genitalia appear to be fully formed and sclerotized and indicate that it is a new species. $P$. webbi geographically occurs between the two most closely related species, $P$. luzonensis and $P$. mutabilis. P. luzonensis occurs on the Philippine island of Luzon lying approximately $400 \mathrm{~km}$ south of Taiwan. P. mutabilis is known from the Ryukus (Tokunoshima, Kunigami and Nago) which lie $\sim 500-600 \mathrm{~km} \mathrm{NE}$ of Taiwan. Small islands occur between the larger islands of Luzon, Taiwan, and the Ryukyus, and thus dispersal via island hopping and subsequent isolation may have promoted speciation in this group.

Goniagnathini

Goniagnathus emeljanovi sp. nov. urn:lsid:zoobank.org:act:7A4C507B-38A1-483F-BA4A-D722D26ECBA4

Fig. 26 A-B, D-K

\section{Diagnosis}

G. emeljanovi can be distinguished from other species of the genus by the unique shapes of the style, connective, and aedeagus. G. punctifer (Walker, 1858) is also recorded from Madagascar, and the new 
species can be distinguished from it by the shorter pygofer, absence of a pygofer process, and absence of paired apical or medial processes of the aedeagus.

\section{Etymology}

This species is named in honor of Dr. A.F. Emeljanov, who has made extensive contributions to the knowledge of the Deltocephalinae.

\section{Type matrial}

\section{Holotype}

§., MADAGASCAR, Diego-Suarez Prov., Parc National Montagne d'Ambre, 1125 m., 29 Jan.-11 Feb. 2001, 12³1'13” S - 49¹0'45” E, malaise trap, R. Harin 'Hala coll., MA-01-01D-03, coll. CAS.

\section{Paratype}

1 q, same data as holotype, but with additional label with collection code: CASLOT 007672, coll. CAS.

\section{Description}

SIZE. $\widehat{o} 5.2 \mathrm{~mm}$, $+5.5 \mathrm{~mm}$.

CoLOR. Generally brownish, mottled with darker brown and ivory markings.

Body. Head slightly wider than pronotum. Crown broad; slightly longer medially than next to eye; glabrous with longitudinal striations; convex; rounded to face. Ocelli on anterior margin of head; $3 \mathrm{x}$ their own diameter from adjacent eye. Frontoclypeus tawny, speckled with dark brown and red dots; slightly longer than wide. Clypellus widening apically. Lorum wider than clypellus at base. Gena with fine erect seta distant from laterofrontal suture. Antennal sockets near lower corners of eyes. Antennal ledges weakly carinate. Antennae short. Pronotum carinate laterally; lateral margin slightly less than $0.5 \mathrm{x}$ width of eye. Forewing with extensive brown irrorate markings; veins whitish, interrupted by brown markings; with crossvein between A1 and claval suture; with A1-A2 crossvein. Protrochanter with stout apical seta. Profemur row AV setae relatively long. Protibia dorsal surface rounded; macrosetae 3+4. Mesotrochanter with stout apical PV and AV setae. Mesofemur row AV with 7-8 relatively long setae. Mesotibia dorsal setae 4+4. Metatibia pecten setae nearly equal in size. Metatarsomere I pecten with 5 platellae; PV seta platellate.

MALE. Pygofer short; rounded; incised dorsally to midlength; with $\sim 8$ long macrosetae near posterior margin; with numerous shorter thick setae posteroventrally; pygofer lobes nearly touching ventrally. Subgenital plates fused; with numerous macrosetae subapically. Connective short; Y-shaped; fused to aedeagus. Style very broad basally; median anterior arm long, extended dorsally to meet connective; preapical lobe knob-like; apophysis with setae basally; apex expanded, with two points; apex textured with several rows of small bumps. Aedeagus very broad at base in ventral view; excavated ventromedially; bifurcate at apex; dorsal margin dentate apically.

Female. Sternite VII undulately excavated. Pygofer with medium-sized macrosetae ventrally and subapically. First valvulae slender; strongly convex; dorsal sculpturing pattern strigate basally, concatenate apically, reaching dorsal margin. Second valvulae slender; with short irregular dorsal teeth and serrations on apical 1/3. Third valvulae with ventroapical row of $\sim 6$ distinct setae. 


\section{Hecalini}

Hecalus hamiltoni sp. nov. urn:1sid:zoobank.org:act:1E2332E7-7260-41B4-81B5-70077EBA59C5

Fig. 28 A-B, D-I

\section{Diagnosis}

Males of $H$. hamiltoni can be distinguished from other species of the genus by the aedeagus with very distinctive long lamellate process arising ventromedially. All other species have shorter processes arising apically or subapically.

\section{Etymology}

This species is named in honor of Dr. K.G.A. Hamilton, who has contributed greatly to the taxonomy of North American Deltocephalinae and whose recent revision of the New World Hecalini facilitated the discovery of this species.

\section{Type material}

\section{Holotype}

ð’, USA, CA, Riverside Co., Rt. 371 mi E jct 79, Aguanga 6, 75 m, N 3327’18” - W 11651’34”, 24 Apr. 1998, RA Rakitov \& CH Dietrich sweeping, 98-035, coll. INHS.

\section{Paratypes}

1 đ̊, USA, CA, San Diego County Rd., S1 mile 15, 1479 m, 3249’33” N - 116²9’45” W, 23 Apr. 1998, C.H. Dietrich, vacuum, 98-031; 1 ภ, 2 + $\odot$ and 1 nymph, USA, CA, San Diego Co., Kitchen Crk. near Cibbets Flat Cmpgd., 3246’54” N - 116²6’39” W, 23 Apr. 1998, C.H. Dietrich, vacuum, 98-029. Two additional nymphs from the last locality are stored in ethanol at INHS. All specimens deposited at INHS.

\section{Description}

SIZE. $\overbrace{}^{\Uparrow}$ 5.7-5.8 mm, $q$ 7.5-8.9 mm.

CoLoR. Generally yellow to orangish-yellow.

BoDy. Head produced; narrower than pronotum. Crown slightly shorter than least width between eyes; texture completely shagreen, basal half more shiny. Anterior margin of head foliaceous; slightly upturned; in male, anterior margin colored white with fuscous above and below. Face of male mostly fuscous, lighter anteriorly and near sutures; face of female yellow. Legs and ventral sclerites of male fuscous to dark brown, of female yellow. Pronotum lateral margin longer than width of eye; carinate. Forewing of male reaching mid-length of pygofer, slightly darkened apically; forewing of female reaching apex of final pregenital tergite, not darkened apically; outer anteapical cell long, expanded apically; with several extra straight costal veins arising from outer anteapical cell. Hindwings of male smoky, of female not strongly colored.

MALE. Pygofer pointed dorsoapically; broadly rounded ventrally; narrowly incised dorsally, incision not reaching midlength of pygofer; with numerous macrosetae and some smaller setae. Subgenital plates long; uniseriate laterally and with some smaller scattered setae and short fine setae near apex. Connective Y-shaped; stem very short. Style very broad basally; preapical lobe distinct, rounded; apophysis slender, pointed apically. Aedeagus laterally compressed; shaft robust, serrate ventrally; gonopore apical; with pair of long lamellate processes arising ventromedially, extending dorsally and recurved; processes serrate ventrally. Segment X basal half sclerotized dorsally and laterally; apical half membranous. With small pair of sclerites in tissue connecting aedeagus and segment X. 
Female. Sternite VII broadly rounded; slightly undulate. Ovipositor protruding from pygofer.

\section{Remarks}

The aedeagal processes became somewhat more coiled after soaking in $\mathrm{KOH}$.

$$
\text { Scaphoideini }
$$

Scaphoideus omani sp. nov. urn:1sid:zoobank.org:act:3CB10731-F785-4BB6-B17D-E5D75DA4E39D

Fig. 47

\section{Diagnosis}

S. omani can be distinguished from other species of the genus by the color pattern and the shape of the aedeagus. At least one other undescribed species with a very similar color pattern is known from sub-Saharan Africa, but differs from S. omani in details of the color pattern, especially on the crown, mesonotum, and base of the forewing, and in the male genitalia.

\section{Etymology}

This species is named in memory of Prof. Paul Wilson Oman, whose seminal work on the New World leafhopper fauna made the present work possible.

\section{Type material}

\section{Holotype}

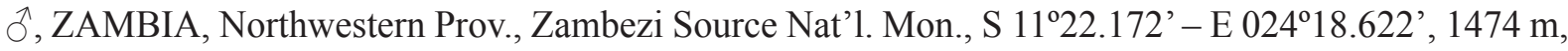
6 Nov. 2007, Hg-vapor light, J.N. Zahniser, ZA-06, coll. INHS.

\section{Paratypes}

$3 \widehat{\partial} \hat{\jmath}$, same data as holotype, coll. INHS; $2 \widehat{\partial}, 1$, ZAMBIA, Northwestern Prov., $\sim 15 \mathrm{~km} \mathrm{~N}$ of Mwinilunga, Lwakera National Forest, 11³4'38.2” S - 024²3'40.1” E, 1445 m, 5 Nov. 2007, Hg-vapor light, J.N. Zahniser, ZA-05, coll. INHS; 1 specimen, sex unknown, RHODESIA, Victoria Falls Nat'1. Park, 3-6 Apr. 1968, Paul Spangler, coll. NMNH.

\section{Description}

SizE. Male 4.8-5.0 mm, female $5.3 \mathrm{~mm}$.

CoLOR. Strikingly colored with contrasting bright yellow or ivory with dark brown areas. Crown bright yellow/cream colored, with irregular brown markings near anterior corners of eyes. Face brown with slight yellow markings. Pronotum and mesonotum brown laterally; bright yellow/cream colored medially, expanded toward apex of mesoscutellum. Forewing brown on basal $1 / 3$; brown patch acuminate apically toward base of middle anteapical cell; hyaline or whitish on apical 2/3 except reflexed costal veins and apical veins tinged with brown and brown spot in 3rd apical cell. Legs and sternites mostly yellow except mesosternum brown; legs with brown pigment at bases of large setae.

BoDy. Head narrower than pronotum; produced somewhat anteriorly; median length about $1.5 \mathrm{x}$ length next to eye. Crown depressed. Anterior margin of head mostly shagreen, glabrous at tip; angled to face. Ocelli fairly large; situated laterally on anterior margin close to eyes. Frontoclypeus very long, narrow; shagreen. Antennal pits large, encroaching onto frontoclypeus. Antennal sockets situated above middle of eye. Antennae long, $1.5 \mathrm{x}$ width of head or longer. Eyes notched along median margin. Clypellus expanded apically. Lorum wider than clypellus at base. Forewing veins R1 and R2+3 reflexed, strongly pigmented; with an extra reflexed costal vein; outer anteapical cell acuminate basally and apically; inner 
anteapical cell open; inner apical cell short, broad, triangular; with crossvein between A1 and claval suture; A1 strongly curved distally, meeting commissural margin nearly at right angle. Profemur row AV setae absent or reduced; intercalary row with $~ 10$ long fine setae; row AM with AM1 only, situated near ventral margin. Protibia dorsal setae 1+4. Mesofemur row AV with numerous short, stout setae. Mesotibia dorsal setae 4+4. Metafemur apical setae 2+2+1. Metatibia row PD setae very long; longest setae nearly equal to or longer than protibia. Metatarsomere I long; apex with 5 platellae; apical row inner seta platellate.

MALE. Pygofer somewhat long, narrow; dorsal incision not reaching midlength; with numerous macrosetae medially on posterior half; posterodorsal apex with vertical row of $\sim 8$ very long macrosetae; internally with sclerotized bars reaching from base of segment $X$ to anteroventral corner of aedeagus. Valve distinctly pointed medially. Subgenital plates long, triangular; with $\sim 3$ macrosetae laterally; with several long thin lateral and apical setae. Connective anterior arms touching apically; stem short; dorsal margin of stem produced in lateral view; fused to pair of processes. Processes of connective fused to each other along basal $2 / 3$; fused to connective; in lateral view extending posterodorsally and curved posteroventrally near apex; processes flared toward apex in ventral view. Style preapical lobe distinct, with several long setae; apophysis long, gracile, with minute texturing. Aedeagus subquadrate in shape; posterior and dorsal margins indented; with flange from dorsoapical corner extending anteroventrally, spade-shaped in posterior view; gonopore on posterior surface near dorsal margin; disassociated from connective and connective processes. Segment X long; lightly sclerotized dorsally at base.

FEMALE. Sternite VII median length longer than width; produced medially; notched apically; with lateral lobes. Sternite VIII very darkly sclerotized medially. Pygofer with numerous ventral macrosetae; apex of each pygofer side with two bunches of 7-8 very long macrosetae; dorsoapical rim with group of 6 long macrosetae. First valvifers closely associate or lightly fused anteriorly; with distinct sclerotized margins. First valvula convex; dorsal sculpturing pattern strigate to reticulate, reaching dorsal margin. Second valvula with small irregular dorsal teeth reaching nearly to midlength; shaft with blunt dorsal tooth.

\title{
Remarks
}

This species is placed in Scaphoideus based on the following characters: forewing outer anteapical cell small, acuminate apically; forewing vein A1 strongly curved apically, meeting commissural margin at nearly right angle; connective with long pair of processes (in this case, processes are fused to each other basally and fused to connective); aedeagus disassociated from connective and connective processes.

\author{
Selenocephalini: Dwightlina \\ Dwightla delongi sp. nov. \\ urn:1sid:zoobank.org:act:D2B3ECC6-5D8E-43A2-8D1A-543D11CABC40
}

Fig. 50 A-B, D-F, H-K

\section{Diagnosis}

D. delongi can be distinguished from other species of Dwightla by the long narrowly pointed pygofer apex (similar in $D$. hercules but lobe is wider in that species) and the aedeagus with a pair of long apical falcate processes directed ventrally.

\section{Etymology}

This species is named after Dwight M. DeLong, author of 86 valid genera and 833 species of Deltocephalinae. 


\section{Type material}

\section{Holotype}

đ, CAMEROON, Southwest, nr. Korup National Park, at bridge, $05^{\circ} 03^{\prime} 02.1^{\prime \prime} \mathrm{N}-08^{\circ} 56^{\prime} 41.0^{\prime \prime} \mathrm{E}, 15-30$ Mar. 2009, J.R. Cryan \& G.J. Svenson, \#8, coll. INHS.

\section{Description}

SIZE. ठ̊ $9.1 \mathrm{~mm}$.

CoLor. General color reddish-brown.

BoDy. Head slightly wider than pronotum. Crown broad; slightly longer medially than next to eye; about $4 \mathrm{x}$ wider than width of eye; texture glabrous with longitudinal striae; depressed preapically; with 8 more or less well defined black maculae. Anterior margin of head with 3 distinct carinae. Ocelli on anterior margin of head; about $2 \mathrm{x}$ their own diameter from adjacent eye. Face light brown; broad. Frontoclypeus shagreen; distinctly broadening dorsally. Clypellus widening apically; somewhat ovoid. Lorum large. Gena wide; with long fine erect seta laterad and distant from frontoclypeal suture. Antennal sockets large; near upper corners of eyes. Antennal ledges distinctly carinate. Antennae somewhat long, about as long as width of head. Pronotum carinate laterally; lateral margin about as long as width of eye; with $\sim 10$ more or less well defined black maculae in a fluctuate transverse line near anterior margin. Forewing with numerous short fine hairs on veins and wing surface; apices folded over, forming pointed apex; medial part of wing apex texture delicate; costal margin whitish, with small black spots near apex; with 1 or 2 crossveins between A1 and claval suture; with A1-A2 crossvein. Profemur row AM with AM1 and 2 or 3 more proximal macrosetae; intercalary row with fine setae apically, apparently with larger setae basally, basal setae continuous with row AV setae, generally in one row but with a few scattered setae; row AV setae long; dorsally with 2 apical and 4-5 more proximal macrosetae. Protibia rounded dorsally; macrosetae $1+7$ (or so). Mesofemur row AV with $\sim 10$ short setae. Mesotibia dorsal setae $\sim 5+7$. Metafemur apical setae 2+2+1. Metatibia row PV macrosetae thick with 1-2 slightly smaller lighter setae between; dorsal surface with supernumerary short stout setae between rows AD and PD; pecten setae nearly equal in length. Metatarsomere I pecten setae with 6 platellae; PV seta platellate.

MALE. Pygofer incised dorsally nearly $2 / 3$ its length; without macrosetae; produced caudoventrally; sharply pointed at apex. Subgenital plate triangular; with scattered macrosetae laterally. Connective broadly Y-shaped; stem very short. Style slender; preapical lobe evident in lateral view; apophysis digitate, turned slightly medially at apex, ventral side with rough texture and subapical small tooth. Aedeagus with pair of long falcate apical processes directed ventrally (holotype with one of these processes broken at base and directed anteriorly). Segment X long; sclerotized laterally and dorsally, and ventrally near apex.

Selenocephalini: Ianeirina

Abimwa knighti sp. nov. urn:Isid:zoobank.org:act:54F1AF2D-EB8D-4E4C-8C5B-FCF0EA93EB1B

Fig. 51

\section{Diagnosis}

A. knighti can be distinguished from other species in the genus by the relatively long and constricted subgenital plate/valve complex; very short aedeagus with one pair of dorsal knob-like processes at apex, and size and shape of the basal processes of the aedeagus. 


\section{Etymology}

The species is named in honor of Dr. W.J. Knight, who has made significant contributions to the knowledge of Old World Deltocephalinae.

\section{Type material}

\section{Holotype}

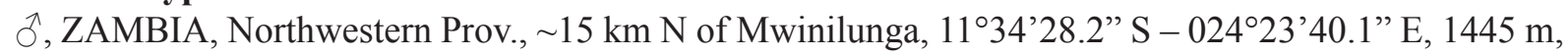
5 Nov. 2007, Hg-vapor light, ZA-05, J.N. Zahniser, coll. INHS.

\section{Paratypes}

$1 \hat{\jmath}, 2$ 우, same data as holotype, coll. INHS. The $\hat{\jmath}$ paratype is completely cleared and DNA was extracted and stored at INHS. Its voucher number is 'DEL 104'. It was included in previous phylogenetic analyses of Deltocephalinae (Zahniser \& Dietrich 2010) listed as "Abimwa n.sp.", and the same data are included in analyses here.

\section{Description}

SizE. $\widehat{O}$ and $+6.3 \mathrm{~mm}$.

CoLoR. General color brown with extensive whitish coloration and small dots.

Body. Head slightly narrower than pronotum. Eye wider than $1 / 2$ width of crown. Crown depressed; texture smooth; sharply angled to face. Anterior margin of head with numerous transverse carinae. Ocelli on face below carinae; about $1.5 \mathrm{x}$ their own width from adjacent eye. Face somewhat darker in color. Frontoclypeus shagreen. Laterofrontal sutures extending beyond ocelli to anterior margin of head. Clypellus expanding apically. Antennal sockets near lower corners of eyes. Antennal ledges carinate. Antennae about as long as width of head. Pronotum carinate laterally; lateral margin less than half width of eye. Forewing whitish hyaline with numerous brown pigment lines and dots; veins brown. Pro-, meso-, and metafemora with 2 broad dark brown longitudinal stripes or blotches, basal stripe or blotch broader than subapical one. Profemur row AM with AM1 and 1 extra macroseta near base of intercalary row; intercalary row with $\sim 5-6$ long fine setae; row AV setae stout. Protibia dorsal macrosetae 3(or 4) +6 . Mesofemur row AV with numerous short stout setae. Mesotibia dorsal setae $\sim 6+6$. Metafemur apical setae $2+2+1$. Metatarsomere I apical setae with 4 platellae; PV seta platellate.

MALE. Pygofer reduced and modified; sclerotized dorsally only as transverse band; with short roundedrectangular lateral lobes without setae; lobes articulating posteriorly with anteroventral lobes of segment X. Subgenital plates fused to valve; plates separated apically; with short fine hairs laterally; with several uniseriate or scattered macrosetae near apex. Valve/plate complex very broad basally, constricted medially. Connective Y-shaped; stem nearly as long as anterior arms; fused to aedeagus. Style small; broadly bilobed basally; lateral anterior arm stalked, knob-like; preapical lobe absent; apophysis short, rounded, blunt. Aedeagus fused to connective; with pair of long widely separated lateral processes arising from base, directed caudodorsally; shaft very short; shaft with pair of short, knob-like processes on dorsal side; gonopore large, apical. Segment X very large, occupying majority of dorsal side of genital capsule; produced anterolaterally into pair of dentate lobes; with pair of medial darkly sclerotized plates arising from inner margin; medial plates with digitate less sclerotized lobes anteriorly; lobes of medial plates articulating with separate small, broad, chevron-shaped sclerotized plate.

FEMALE. Sternite VII broadly excavated posteriorly. Pygofer with numerous setae ventroapically. First valvifers fused anterodorsally. First valvula convex; dorsal sculpturing pattern strigate, reaching dorsal 
margin. Second valvula with small knob-like dorsal teeth on apical $1 / 3$ to $1 / 4$; teeth not serrate; with blunt median dorsal tooth. Third valvula with $\sim 8$ distinct close-set setae ventroapically.

\title{
Remarks
}

Previous descriptions of species in this genus did not mention the inner plate-like processes of the male segment $\mathrm{X}$ and the short median plate described here, so it is unknown if they are unique to this species.

\author{
Selenocephalini: Selenocephalina \\ Gannia viraktamathi $\mathrm{sp}$. nov. \\ urn:Isid:zoobank.org:act:76324C0E-0625-483B-ACC6-AABCF336CE49
}

Fig. 52 A-B, D-K

\section{Diagnosis}

G. viraktamathi can be distinguished from other species of the genus by the unique shapes of the aedeagus, ventral processes of the aedeagus, the pygofer, and male segment $\mathrm{X}$.

\section{Etymology}

This species is named in honor of Dr. C.A. Viraktamath, a leading expert on Oriental Cicadellidae.

\section{Type material}

\section{Holotype}

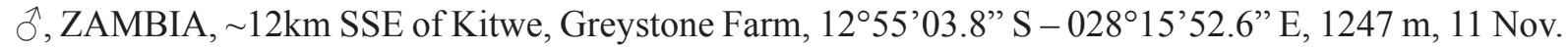
2007, Hg-vapor light, ZA-09, J.N. Zahniser, coll. INHS.

\section{Paratypes}

$1 \AA, 1$, same data as holotype, coll. INHS.

\section{Description}

SIZE. §ै 6.5-7.0 mm, $+8.2 \mathrm{~mm}$.

COLOR. General color rusty brown to ochraceous with extensive dark brown mottled markings.

BoDy. Head wider than pronotum. Crown broad, more than $2 \mathrm{x}$ width of eye; not produced; barely longer medially than next to eye; texture glabrous with slight longitudinal or radial striations; sharply angled to face. Anterior margin of head with 3 distinct parallel carinae between eyes; with a fourth less distinct carina below distinct carinae. Ocelli large; close to eyes; less than 1 diameter width from adjacent eye. Face broad. Frontoclypeus light tannish in color, with dark brown or black beneath anterior carinae; widening dorsally; depressed below anterior margin of head; texture shagreen. Clypellus widening apically. Lorum large. Gena wide; with fine erect seta distant from laterofrontal suture. Antennal sockets near middle of eyes. Antennal ledges not carinate; apparent as small bulges. Antennae shorter than width of head. Forewing veins with short fine hairs. Forewing with crossvein between A1 and claval suture; with A1-A2 crossvein. Profemur tannish in color, with dark brown or black ventrally near apex and along intercalary row and spots of coloration dorsally near apex on anterior side; row AM with AM1 and with a smaller seta more proximally near base of intercalary row; intercalary row with $\sim 8-10$ fine setae; row AV with $\sim 6-9$ relatively long thick setae. Protibia dorsal macrosetae $4+4$. Mesofemur row $\mathrm{AV}$ with $\sim 8$ short stout setae; row $\mathrm{PV}$ with distinct somewhat longer setae; anterior side with extra setae near apex. Mesotibia dorsal setae 4(or 5) +5 . Metafemur apical setae 2+2+1. Metatarsomere I apex with 6 platellae; PV seta platellate. 
MaLE. Pygofer not strongly incised dorsally; short; rounded; ventroapically with small lobate extension; without macrosetae; with numerous short setae ventrally. Valve large; triangular. Subgenital plates somewhat long; triangular; lateral margin convex; with numerous macrosetae scattered near lateral margin. Connective short; U-shaped; without stem; with posterior angles sharp; articulating with ventral aedeagal processes at meeting point of two processes. Style elongate; narrow; preapical lobe squarish; apophysis digitate, slightly bent, with rough stippled texture medially. Aedeagus with socle expanded; anteroventral corners extended ventrally and articulating with ventral processes; base of aedeagus squarish with posteroventral corner sharp, with dorsal margin notched; shaft curved; gonopore subapical on ventral side. Ventral processes of aedeagus extending from anteroventral lobes of socle of aedeagus, meeting ventrally, then separating and continuing as long skinny sinuous processes to near top of aedeagus. Segment X very large; distinctly protruding from pygofer; sclerotized for long length laterally and as broad transverse band dorsally; ventroapically with several short, sharp teeth.

FEMALE. Sternite VII slightly excavated, posterior margin broadly parenthesis-shaped. First valvifers fused anterodorsally. First valvula convex; dorsal sculpturing strigate, reaching dorsal margin. Second valvula broad, tapering apically; with small irregular teeth on apical 1/3; with blunt dorsal median tooth. Third valvulae pigmented ventrally; with numerous short setae ventrally in one row and with other scattered setae.

\section{Stenometopiini}

Doratulina dmitrievi sp. nov. urn:1sid:zoobank.org:act:D44FB522-2ED8-4B79-97B7-FCA0EB60359E Fig. 54 A-B, D-J

\section{Diagnosis}

Other than the four species of the distinctive genus Hodoedocus Jacobi, 1910, there are three described species of Stenometopiini from Africa: Doratulina sobrina (Melichar, 1912) from Chad, Gabon, the Republic of the Congo, and Central African Republic; Stirellus flavovirescens (Stål, 1855) from South Africa; and S. sagittarius (Naudé, 1926) from South Africa. The new species does not conform to any of their descriptions.

\section{Etymology}

This species is named for Dr. D.A. Dmitriev in recognition of his contributions to the understanding of the morphology of the nymphs of Deltocephalinae and for his contributions to the 3I database and interactive key to Deltocephalinae.

\section{Type material}

\section{Holotype}

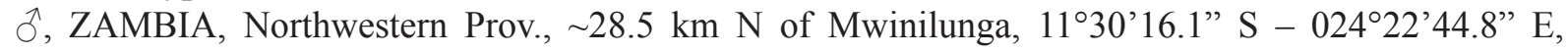
Chitunta plain grassland, 1384 m, 4-7 Nov. 2007, ZA-03, sweep, J.N. Zahniser, coll. INHS.

\section{Paratypes}

$9 \widehat{\diamond} \widehat{\partial}, 6$ 우, same data as holotype. All specimens deposited at INHS.

\section{Description}

SIZE. §̊ 2.9-3.2 mm, क 3.2-3.5 mm.

CoLor. General color light fuscous to dark brown or black. Males usually more darkly colored than females. 
BoDy. Crown shagreen to base; coronal suture lightly colored; with pair of small ovoid dark markings near base. Frontoclypeus fuscous with dark brown arcs from lateral frontal suture reaching only part way across. Clypellus parallel-sided; extending slightly beyond normal curve of gena. Gena with large dark brown spot below antennal bases; with small dark brown spot below eye. Pronotum, forewings, and abdomen fuscous to dark brown or black dorsally; laterally with bright yellow or greenish stripe. Forewings brachypterous, exposing 4 pregenital tergites; venation reticulated. Profemur row AM with AM1 and with an additional more proximal macroseta; intercalary row with a few fine setae; row AV with many close-set, short stout setae. Protibia dorsal macrosetae $1+3$. Mesotibia dorsal macrosetae $3+3$. Metafemur apex macrosetae $2+1$ or $2+1+1$; with several smaller scattered setae.

MALE. Pregenital sternite lightly sclerotized on apical half. Pygofer dorsally incised to base; long, sloping caudoventrally; apex subquadrate; with few macrosetae dorsally; with group of short setae ventrally; with short tooth on apical half near ventral margin; with median lightly colored stripe separating dorsal and ventral parts; apex darkly colored. Segment X long; sclerotized dorsally and laterally. Valve long, triangular. Subgenital plates rounded laterally; macrosetae uniseriate laterally. Connective Y-shaped; anterior arms with small lateral lobes; stem nearly as long as anterior arms; stem divided for most of length. Style narrow; median anterior arm somewhat produced anteriorly; apophysis digitate. Aedeagus with narrowly triangular base; flagellum long, parallel to base, about $2 \times$ longer than longest length of base.

Female. Sternite VII slightly notched medially. Pygofer with few apical macrosetae.

\section{Acknowledgements}

We sincerely thank Dmitri Dmitriev (INHS) for his assistance with the 3I database and for helpful discussion regarding the taxonomy of Deltocephalinae. We thank Stuart McKamey (NMNH) for providing initial lists of taxonomic names that were used as the basis for the nomenclatural data entered into the database. We are grateful to the following curators for assisting with museum visits and loans that were instrumental in this project: Mick Webb (BMNH), Adeline Soulier-Perkins (MNHN), Jerome Constant (IRSNB), Eliane de Coninck (RMCA), Stuart McKamey (NMNH), Randall Schuh (AMNH), and Luciana Musetti (OSUC). For providing specimens used in the phylogenetic analyses, we are grateful to Mike Wilson, Roman Rakitov, Michael Stiller, Mike Irwin, Terry Erwin, Adam Wallner, Vinton Thompson, Daniela Takiya, Murray Fletcher, Mick Webb, Charles Bartlett, Andrew Deans, Norman Johnson, Luciana Musetti, Mike Sharkey, Norman Penny, and Gordon Bennett. We thank Allison Keever, Minerva Dorantes, Elizabeth Gonzalez Maza, and Stephanie Tham for their assistance in photographing leafhopper specimens, editing images, and entering data into the database. We gratefully acknowledge that this work was supported by NSF grant \#DEB 08-41612 ARRA.

\section{References}

Anufriev G.A. \& Emeljanov A.F. 1988. Suborder Cicadinea - (Auchenorrhyncha). In: Lehr P.A. (ed.) Keys to the Insects of the Far East of the USSR. Volume II: 1-10. Nauka Publishing House, Leningrad.

Barnett D.E. 1977. A revision of Nearctic species of the genus Scaphoideus (Homoptera: Cicadellidae). Transactions of the American Entomological Society 102: 485-593.

Barnett D.E. 1979. Seven new North American species of Scaphoideus Uhler (Homoptera: Cicadellidae). Journal of the Kansas Entomological Society 52 (3): 471-482.

Barnett D.E. 1980. Seven new African species of Scaphoidophyes Kirkaldy (Homoptera: Cicadellidae). Proceedings of the Washington Entomological Society 82 (3): 435-446.

Beamer R.H. 1940. The genus Cochlorhinus Uhler and some related genera of leafhoppers (HomopteraCicadellidae). Journal of the Kansas Entomological Society 13: 51-57. 
Beamer R.H. \& Lawson P.B. 1938. The genus Acinopterus (Homoptera, Cicadellidae). Annals of the Entomological Society of America 31: 476-488.

Bennett G.M. \& O'Grady P.M. 2011. Review of the native Hawaiian leafhopper genus Nesophrosyne (Hemiptera: Cicadellidae: Deltocephalinae) with description of eight new species associated with Broussaisia arguta (Hydrangeaceae). Zootaxa 2805: 1-25.

Blocker H.D. 1967. Classification of the western hemisphere Balclutha (Homoptera: Cicadellidae). Proceedings of the United States National Museum 122: 1-55.

Blocker H.D. 1983. Classification and proposed phylogeny of the Neotropical leafhopper genus Icaia Linnavuori. Entomography 2: 77-87.

Blocker H.D. \& Fang Q. 1992. A synopsis of the Faltala Oman leafhopper group (Homoptera: Cicadellidae) with descriptions of new taxa. Journal of the Kansas Entomological Society 65 (3): 341 346.

Blocker H.D., Fang Q. \& Black IV W.C. 1995. Review of Nearctic Deltocephalus-like leafhoppers (Homoptera: Cicadellidae). Annals of the Entomological Society of America 88 (3): 294-315.

Blocker H.D. \& Johnson J.W. 1988a. Classification of the subgenus Athysanella, genus Athysanella Baker (Homoptera, Cicadellidae, Deltocephalinae). Great Basin Naturalist Memoirs 12: 18-42.

Blocker H.D. \& Johnson J.W. 1988b. Subgenera of the genus Athysanella Baker (Homoptera: Cicadellidae: Deltocephalinae) and a proposed phylogeny. In: Vidano C. \& Arzone A. (eds) Proceedings of the 6th International Auchenorrhyncha Congress, September 1987: 39-46. Torino, Italy.

Blocker, H.D. \& Johnson J.W. 1990a. Classification of Athysanella (Gladionura) (Homoptera: Cicadellidae: Deltocephalinae). Journal of the Kansas Entomological Society 63 (1): 9-45.

Blocker H.D. \& Johnson J.W. 1990b. Classification of Athysanella (Amphipyga) (Homoptera: Cicadellidae: Deltocephalinae). Journal of the Kansas Entomological Society 63 (1): 101-132.

Blocker H.D. \& Johnson J.W. 1990c. Classification of five subgenera of Athysanella (Homoptera: Cicadellidae: Deltocephalinae). Journal of the Kansas Entomological Society 63 (2): 304-315.

Boulard M. 1971. Une tribu nouvelle pour la faune africaine des Homoptères Cicadellidae. Bulletin de l'Institut Fondamental d'Afrique Noire. Serie A. Sciences Naturelles 33 (3): 712-717.

Bremer K. 1988. The limits of amino acid sequence data in angiosperm phylogenetic reconstruction. Evolution 42: 795-803.

Chen X.-S., Li Z.-Z. \& Yang L. 2007. Oriental bamboo leafhoppers: revision of Chinese species of Mohunia (Hemiptera: Cicadellidae: Mukariinae) with descriptions of new genera and new species. Annals of the Entomological Society of America 100 (3): 366-374. http://dx.doi.org/10.1603/00138746(2007)100[366:OBLROC]2.0.CO;2

Chen X.-S., Li Z.-Z. \& Yang L. 2008. Oriental bamboo leafhoppers: A new genus and two new species of Mukariinae (Hemiptera: Cicadellidae) from Southwest China and notes on related group. Annales de la Société Entomologique de France 44 (3): 301-307.

Chen X.-S., Liang A.-P. \& Li Z.-Z.. 2009. A new species of bamboo leafhopper genus Mukaria (Hemiptera, Cicadellidae, Mukariinae) from Guangdong, China. Acta Zootaxonomica Sinica 34 (1): 144-147.

Crowder H.W. 1952. A revision of some phlepsiuslike genera of the tribe Deltocephalini (Homoptera, Cicadellidae) in North America north of Mexico. University of Kansas Science Bulletin 35: 309-541. 
Cwikla P.S. 1988a. A systematic study of the leafhopper genus Chlorotettix (Homoptera: Cicadellidae). Ph.D. dissertation, Ohio State University, Columbus, Ohio.

Cwikla P.S. 1988b. A new genus, Ileopeltus, related to Chlorotettix (Homoptera: Cicadellidae). Great Basin Naturalist Memoirs 12: 43-60.

Cwikla P.S. \& Blocker H.D. 1981. Neotropical genera of Deltocephalinae not included in Linnavuori's 1959 key. Bulletin of the Entomological Society of America 27 (3): 170-178.

Cwikla P.S. \& Ma N. 1986. A new species of Cariancha Oman (Homoptera: Cicadellidae: Deltocephalinae) with a discussion on the tribal placement of the genus. Journal of the New York Entomological Society 94 (1): 115-117.

Dai W. \& Dietrich C.H. 2011. Review of the Old World leafhopper genus Scaphoidella Vilbaste (Hemiptera: Cicadellidae: Deltocephalinae), with description of ten new species from Thailand and Vietnam. Annales de la Société Entomologique de France 47 (3-4): 457-473.

Dai W., Dietrich C.H. \& Zhang Y. 2011. Review of the Oriental leafhopper genus Lampridius (Hemiptera: Cicadellidae: Deltocephalinae), with description of a related new genus. Zoological Science 28: 380387. http://dx.doi.org/10.2108/zsj.28.380

Dai W., Cui W., Xiao B. \& Zhang Y. 2010. A new genus and species of Old World Opsiini (Hemiptera: Cicadellidae: Deltocephalinae), with a key to genera and species checklist for the tribe. Zootaxa 2607: 55-68.

Dai W., Viraktamath C.A., Zhang Y. \& Webb M.D. 2009. A review of the leafhopper genus Scaphotettix Matsumura (Hemiptera: Cicadellidae: Deltocephalinae), with description of a new genus. Zoological Science 26: 656-663. http://dx.doi.org/10.2108/zsj.26.656

Dash P.C. \& C.A. Viraktamath. 2001. Deltocephaline leafhopper genus Goniagnathus (Hemiptera: Cicadellidae) in the Indian subcontinent with descriptions of four new species. Journal of the Bombay Natural History Society 98 (1): 62-79.

Davies D.M. 1987. Athysanini (Homoptera: Cicadellidae) described from southern Africa. I. The genera Bonaspeia Linnavuori and Curvostylus gen. nov. Phytophylactica 19: 437-452.

Davies D.M. 1988a. Athysanini (Homoptera: Cicadellidae) described from southern Africa. II. The genera Caffrolix Linnavuori, Colistra gen. nov., and Kimbella gen. nov. Phytophylactica 20: 203-217.

Davies D.M. 1988b. Leafhoppers (Homoptera: Cicadellidae) associated with the Restionaceae. II. The tribes Athysanini and Paralimnini (Euscelinae). Journal of the Entomological Society of Southern Africa 51 (1): 65-80.

DeLong D.M. 1937. Some new species of Ballana (Homoptera, Cicadellidae). The Ohio Journal of Science 37: 105-124.

DeLong D.M. 1938. The genus Phlepsius. A study of the North American species with special reference to the characters of the male genitalia. Lloydia 1:232-252.

DeLong D.M. 1939. Fauna Mexicana. II. (Insecta Homoptera, familia Cicadellidae, genus Phlepsius). Los Phlepsidos (Phlepsius y Texananus) de Mexico (Homoptera-Cicadellidae). [Mexico City]. Anales de la Escuela Nacional de Ciencias Biologicas 1: 379-405.

DeLong D.M. 1945. The genus Chlorotettix (Homoptera-Cicadellidae) in Mexico. Lloydia 8: 1-30.

DeLong D.M. 1948. The leafhoppers, or Cicadellidae, of Illinois. (Eurymelinae-Balcluthinae). Bulletin of the Illinois Natural History Survey 24 (2): 94-376. 
DeLong D.M. 1964. A monographic study of the North American species of the genus Ballana. The Ohio Journal of Science 64 (5): 305-370.

DeLong D.M. \& Thambimuttu C.C. 1973. Three closely related new genera and five new species of short-winged Chilean leafhoppers (Homoptera: Cicadellidae). Florida Entomologist 56: 165-172.

Dietrich C.H. 1999. The role of grasslands in the diversification of leafhoppers (Homoptera: Cicadellidae): a phylogenetic perspective. In: Warwick C. (ed.) Proceedings of the Fifteenth North American Prairie Conference: 44-49. Bend, Oregon.

Dietrich C.H. 2004. Phylogeny of the leafhopper subfamily Evacanthinae with a review of Neotropical species and notes on related groups (Hemiptera: Membracoidea: Cicadellidae). Systematic Entomology 29 (4): 455-487. http://dx.doi.org/10.1111/j.0307-6970.2004.00250.x

Dietrich C.H. \& Dmitriev D.A. 2003. Reassessment of the leafhopper tribes Koebeliini Baker and Grypotini Haupt (Hemiptera: Cicadellidae). Annals of the Entomological Society of America 96 (6): 766-775. http://dx.doi.org/10.1603/0013-8746(2003)096[0766:ROTLTK]2.0.CO;2

Dietrich C.H. \& Rakitov R.A. 2002. Some remarkable new deltocephaline leafhoppers (Hemiptera: Cicadellidae: Deltocephalinae) from the Amazonian rainforest canopy. Journal of the New York Entomological Society 110 (1): 1-48.

Dietrich C.H., Rakitov R.A., Holmes J.L. \& Black W.C. 2001. Phylogeny of the major lineages of Membracoidea (Insecta: Hemiptera: Cicadomorpha) based on 28S rDNA sequences. Molecular Phylogenetics and Evolution 18 (2): 293-305. http://dx.doi.org/10.1006/mpev.2000.0873

Dietrich C.H., Whitcomb R.F. \& Black IV W.C. 1997. Phylogeny of the grassland leafhopper genus Flexamia (Homoptera: Cicadellidae) based on mitochondrial DNA sequences. Molecular Phylogenetics and Evolution 8: 139-149. http://dx.doi.org/10.1006/mpev.1997.0415

Dietrich C.H., Fitzgerald S.J., Holmes J.L., Black IV W.C. \& Nault L.R. 1998. Reassessment of Dalbulus leafhopper (Homoptera: Cicadellidae) phylogeny based on mitochondrial DNA sequences. Annals of the Entomological Society of America 91 (5): 590-597.

Distant W.L. 1908. Rhynchota - Homoptera. In: Bingham C.T. (ed.) The Fauna of British India, including Ceylon and Burma. Volume 4. Taylor and Francis, London.

Distant W.L. 1918. Rhynchota. Homoptera: Appendix. Heteroptera: Addenda. In: Shipley A. E. \& Marshall G. A. K. (eds) The Fauna of British India, including Ceylon and Burma. Volume 7. Taylor and Francis, London.

Dmitriev D.A. 2001. Larvae of some species of the subfamily Eupelicinae (Homoptera: Cicadellidae). Zoosystematica Rossica 9 (2): 353-357.

Dmitriev D.A. 2002. Larvae of the leafhopper subfamily Deltocephalinae (Homoptera, Cicadellidae) from European part of Russia and adjacent territories. I. A key to tribes and review of the tribes Drabescini, Scaphytopiini, Hecalini, Limotettigini, and Opsiini. Entomologicheskoe Obozrenie 81 (3): 627-661. [In Russian, translated into English in Entomological Review 82 (8): 975-1002]

Dmitriev D.A. 2003. Larvae of the leafhopper subfamily Deltocephalinae (Homoptera, Cicadellidae) from European part of Russia and adjacent territories. II. Tribes Grypotini, Selenocephalini, Goniagnathini, Fieberiellini, Tetartostylini, Macrostelini, and Doraturini. Entomologicheskoe Obozrenie 82(3): 650686. [In Russian, translated into English in Entomological Review 83 (6): 665-695]

Dmitriev D.A. 2004a. Nymphs of some species of the tribes Drabescini and Paraboloponini with a proposed synonymy of Paraboloponini with Drabescini (Hemiptera: Cicadellidae: Deltocephalinae). Oriental Insects 38: 235-244. http://dx.doi.org/10.1080/00305316.2004.10417390 
Dmitriev D.A. 2004b. Larvae of leafhoppers of the subfamily Deltocephalinae (Homoptera, Cicadellidae) from European Russia and adjacent territories: III. Tribes Deltocephalini, Stirellini, and Paralimnini. Entomological Review 84 (1): 31-53.

Dmitriev D.A. 2006a. 3I, a new program for creating Internet-accessible interactive keys and taxonomic databases and its application for taxonomy of Cicadina (Homoptera). Russian Entomological Journal 15 (3): 263-268.

Dmitriev V.D.[sic] 2006b. Larvae of leafhoppers of the subfamily Deltocephalinae (Homoptera, Cicadellidae) of European Russia and adjacent territories: IV. Tribe Athysanini. Entomological Review 86 (1): 11-48. http://dx.doi.org/10.1134/S0013873806010027

Dmitriev D.A. 2009. Nymphs of some Nearctic leafhoppers (Homoptera, Cicadellidae) with description of a new tribe. Zookeys: 29: 13-33. http://dx.doi.org/10.3897/zookeys.29.223

Dominguez E. \& Godoy C. 2010. Taxonomic review of the genus Osbornellus Ball (Hemiptera: Cicadellidae) in Central America. Zootaxa 2702: 1-106.

Duan Y., Zhang Y. \& Webb M.D. 2009. Review of the grassland leafhopper subgenus Leofa (Prasutagus) Distant (Hemiptera: Cicadellidae: Deltocephalinae: Chiasmini). Zootaxa 1932: 35-43.

Duan Y. \& Zhang Y. 2012a. New records of the grassland leafhopper genus Doratura Sahlberg (Hemiptera: Cicadellidae: Deltocephalinae: Chiasmini) from China. Zootaxa 3175: 54-62.

Duan Y. \& Zhang Y. 2012b. Redescription of the grassland leafhopper genus Doraturopsis Lindberg (Hemiptera: Cicadellidae: Deltocephalinae: Chiasmini) with description of a new genus and species from China. Zootaxa 3177: 24-32.

Duan Y. \& Zhang Y. 2012c. A taxonomic review of the grassland leafhopper genus Aconurella Ribaut (Hemiptera: Cicadellidae: Deltocephalinae: Chiasmini) from China with the description of two new species. Zootaxa 3397: 28-44.

d'Urso V. 1992. Taxonomical notes on Paradorydium paradoxum (Herrich-Schaffer, 1837) and Paradorydium lanceolatum (Burmeister, 1839) (Homoptera, Auchenorrhyncha). Deutsche Entomologische Zeitschrift 39 (1-3): 55-63. http://dx.doi.org/10.1002/mmnd.19920390104

Edgar R.C. 2004. MUSCLE: multiple sequence alignment with high accuracy and high throughput. Nucleic Acids Research 32 (5): 1792-1797. http://dx.doi.org/10.1093/nar/gkh340

Emeljanov A.F. 1962. New tribes of the leafhopper subfamily Euscelinae (Auchenorrhyncha, Cicadellidae). Entomologicheskoe Obozrenie 41: 388-397. [In Russian with English summary, translation in Entomological Review 41 (2): 236-240]

Emeljanov A.F. 1966. On the tribe Stirellini trib.n. and its taxonomic position (Homoptera, Cicadellidae). Zoologicheskii Zhurnal 45: 609-610. [In Russian with English summary]

Emeljanov A.F. 1967. Suborder Cicadinea (Auchenorrhyncha). In: Bei-Bienko G.Y. (ed.) Keys to the Insects of the European USSR. I. Apterygota, Palaeoptera, Hemimetabola: 421-551. Israel Program for Scientific Translations, Jerusalem.

Emeljanov A.F. 1968. Notes on the tribe Stirellini (Homoptera, Cicadellidae). Zoologicheskii Zhurnal 47: 249-253.

Emeljanov A.F. 1999. A key to genera of the subfamily Deltocephalinae s.l. (Homoptera, Cicadellidae) from Kazakhstan, Middle Asia, and Mongolia, with description of new genera and subgenera. Entomological Review 79: 547-562.

Evans J.W. 1954. Les Cicadellidae de Madagascar (Homopteres). Mémoires de l'Institut Scientifique de Madagascar (Série E) 4: 87-137. 
Evans J.W. 1966. The leafhoppers and froghoppers of Australia and New Zealand (Homoptera: Cicadelloidea and Cercopoidea). Memoirs of the Australian Museum 12: 1-347.

Evans J.W. 1972a. Characteristics and relationships of Penthimiinae and some new genera and new species from New Guinea and Australia; also new species of Drabescinae from New Guinea and Australia (Homoptera: Cicadellidae). Pacific Insects 14 (1): 169-200.

Evans J.W. 1972b. Some leafhoppers from New Guinea, Australia, and Thailand belonging to the subfamily Jassinae and a new genus from New Guinea referred to a new subfamily, the Acostemminae (Homoptera: Cicadellidae). Pacific Insects 14 (4): 647-662.

Fang Q., Black IV W.C., Blocker H.D. \& Whitcomb R.F. 1993. A phylogeny of New World Deltocephalus-like leafhopper genera based on mitochondrial 16S ribosomal DNA sequences. Molecular Phylogenetics and Evolution 2 (2): 119-131. http://dx.doi.org/10.1006/mpev.1993.1012

Fang, Q., Blocker H.D. \& Black IV W.C. 1995. Cladistic analysis of Nearctic Deltocephalus-like leafhoppers (Homoptera: Cicadellidae) using morphological and molecular characters. Annals of the Entomological Society of America 88 (3): 316-323.

Fletcher M.J. \& Zahniser J.N. 2008. The first record of Goniagnathini from Australia with description of four new species of Goniagnathus Fieber (Hemiptera: Cicadellidae: Deltocephalinae). Zootaxa 1692: 43-54.

Freytag P.H. 2008. Review of the genus Osbornellus Ball (Hemiptera: Cicadellidae: Deltocephalinae) from Hispaniola, including fifteen new species. Entomological News 119 (2): 131-150. http://dx.doi. org/10.3157/0013-872X(2008)119[131:ROTGOB]2.0.CO;2

Ghauri M.S.K. 1966. Revision of the genus Orosius Distant (Homoptera: Cicadelloidea). Bulletin of the British Museum (Natural History), Entomology 18 (7): 231-252.

Hadley A. 2010. CombineZP. Available from http://www.hadleyweb.pwp.blueyonder.co.uk/CZP/ Installation.htm [accessed May 11, 2013]

Hall T.A. 1999. BioEdit: a user-friendly biological sequence alignment editor and analysis program for Windows 95/98/NT. Nucleic Acids Symposium Series 41: 95-98.

Hamilton K.G.A. 1975a. Revision of the genera Paraphlepsius Baker and Pendarus Ball (Rhynchota: Homoptera: Cicadellidae). Memoirs of the Entomological Society of Canada 96: 1-129.

Hamilton K.G.A. 1975b. Review of the tribal classification of the leafhopper subfamily Aphrodinae (Deltocephalinae of authors) of the Holarctic region (Rhynchota: Homoptera: Cicadellidae). The Canadian Entomologist 107: 477-498.

Hamilton K.G.A. 1994. Evolution of Limotettix Sahlberg (Homoptera-Cicadellidae) in peatlands, with descriptions of new taxa. Memoirs of the Entomological Society of Canada 169: 111-133.

Hamilton K.G.A. 2000. Five genera of New-World "Shovel-Headed" and "Spoon-Bill" leafhoppers (Hemiptera: Cicadellidae: Dorycephalini and Hecalini). The Canadian Entomologist 132 (4): 429-503. http://dx.doi.org/10.4039/Ent132429-4

Hayashi M. 1996. Occurrence of Mukariinae (Homoptera, Cicadellidae) in Japan, with description of a new species. Japanese Journal of Entomology 64 (1): 122-130.

Hepner L.W. 1947. A revision of the tribe Scaphytopiini (Homoptera, Cicadellidae) in North America. University of Kansas Science Bulletin 31 (2): 413-541.

Huelsenbeck J.P. \& Rannala B. 2004. Frequentist properties of Bayesian posterior probabilities of phylogenetic trees under simple and complex substitution models. Systematic Biology 53: 904-913. http://dx.doi.org/10.1080/10635150490522629 
International Commission on Zoological Nomenclature (ICZN). 1999. International Code of Zoological Nomenclature. The International Trust for Zoological Nomenclature, London.

Jacobi A. 1912. Homoptera. Wissenschaftliche Ergebnisse der Deutsch-Zentral-Afrika-Expedition. 1907-1908. Volume 4: 19-41. Klinkhardt \& Biermann, Leipzig.

Kamitani S. 1999. The phylogeny of the genera in the tribes Deltocephalini, Paralimnini, and their allies (Homoptera, Cicadellidae, Deltocephalinae). Esakia 39: 65-108.

Khatri, I. \& Webb M.D. 2011. On the identity of Benglebra Mahmood \& Ahmad, and other Mukariini (Hemiptera: Cicadellidae: Deltocephalinae) from Bangladesh and Pakistan. Zootaxa 2885: 14-22.

Kirkaldy G.W. 1910. Hemiptera. Supplement to Fauna Hawaiiensis, or the Zoology of the Sandwich (Hawaiian) Isles: 531-599.

Kirschbaum C.L. 1868. Die Cicadinen der Gegend von Wiesbaden und Frankfurt a.M. nebst einer Anzahl neuer oder schwer zu unterscheidender Arten aus anderen Gegenden Europas. Tabellarisch beschrieben. Jahrbücher des Vereins für Naturkunde im Herzogthum Nassau 21-22: 1-202.

Knight W.J. 1970a. A revision of the genus Hishimonus Ishihara (Hom., Cicadellidae). Suomen Hyonteisteellinen Aikakauskirja 36 (3): 125-139.

Knight W.J. 1970b. Two new genera of leafhoppers related to Hishimonus Ishihara (Hom., Cicadellidae). Suomen Hyonteisteellinen Aikakauskirja 36 (4): 174-182.

Knight W.J. 1974. Evolution of the Holarctic leafhopper genus Diplocolenus Ribaut, with descriptions and keys to subgenera and species (Homoptera: Cicadellidae). Bulletin of the British Museum (Natural History), Entomology 29 (7): 357-413.

Knight W.J. 1987. Leafhoppers of the grass-feeding genus Balclutha (Homoptera, Cicadellidae) in the Pacific region. Journal of Natural History 21: 1173-1224.

Knight W.J. \& Fletcher M.J. 2007. A new deltocephaline leafhopper genus, Melanetettix, from Melanesia (Hemiptera: Cicadellidae: Deltocephalinae). Systematics and Biodiversity 5 (2): 199-222. http:// dx.doi.org/10.1017/S1477200006002027

Knight W.J. \& Webb M.D. 1993. The phylogenetic relationships between virus vector and other genera of macrosteline leafhoppers, including descriptions of new taxa (Homoptera: Cicadellidae: Deltocephalinae). Systematic Entomology 18: 11-55. http://dx.doi.org/10.1111/j.1365-3113.1993. $\underline{\text { tb00653.x }}$

Knight W.J. \& Webb M.D. 2002. A redescription of the type species of Scaphotettix viridis Matsumura (Insecta: Hemiptera: Cicadellidae: Deltocephalinae). Reichenbachia 34 (31): 267-269.

Li Z. \& Chen S. 1998. Nirvaninae from China (Homoptera: Cicadellidae). Guizhou Science and Technology Publishing House, Guiyang.

Linnavuori R. 1957. The Neotropical Hecalinae (Hom. Cicadellidae). Suomen Hyonteistieteellinen Aikakaukirja 23: 133-143.

Linnavuori R. 1959. Revision of the Neotropical Deltocephalinae and some related subfamilies (Homoptera). Annales Zoologici Societatis Zoologicae Botanicae Fennicae 'Vanamo’ 20: 1-370.

Linnavuori R. 1961. Hemiptera (Homoptera): Cicadellidae. In: Hanström B., Brinck P. \& Rudebeck G. (eds) South African Animal Life 8: 452-486.

Linnavuori R. 1969. Contribution à la faune du Congo (Brazzaville). Mission A. Villiers et A. Descarpentries XCIII. Hémiptères Hylicidae et Cicadellidae. Bulletin de l'Institut Fondamental d'Afrique Noire (Series A) 31(4): 1129-1185. 
Linnavuori R. 1975. Revision of the Cicadellidae (Homoptera) of the Ethiopian region 3. Deltocephalinae, Hecalini. Acta Zoologica Fennica 143: 1-37.

Linnavuori R. 1977. Revision of the Ethiopian Cicadellidae (Hemiptera-Homoptera): Penthimiinae. Études du Continent Africain, Fascicule 4: 1-76.

Linnavuori R. 1978a. Revision of the African Cicadellidae (subfamilies Nioniinae, Signoretiinae and Drabescinae) (Homoptera, Auchenorrhyncha). Annales Entomologici Fennici 44(2): 33-48.

Linnavuori R. 1978b. Revision of the Ethiopian Cicadellidae (Homoptera) Paraboloponinae and Deltocephalinae: Scaphytopiini and Goniagnathini. Revue de Zoologie et de Botanique Africaines 92 (2): 457-500.

Linnavuori R. \& DeLong D.M. 1978c. Genera and species of Hecalini (Homoptera, Cicadellidae, Deltocephalinae) known to occur in Mexico. Annales Entomologici Fennici 44: 48-53.

Linnavuori R. 1979a. Revision of the African Cicadellidae (Homoptera Auchenorrhyncha) Part I. Revue de Zoologie et de Botanique Africaines 93 (3): 647-747.

Linnavuori R. 1979b. Revision of the African Cicadellidae (Homoptera Auchenorrhyncha) Part II. Revue de Zoologie et de Botanique Africaines 93 (4): 929-1010.

LinnavuoriR. \&Al-Ne'amy K.T. 1983. Revision of the African Cicadellidae (subfamily Selenocephalinae) (Homoptera, Auchenorrhyncha). Acta Zoologica Fennica 168: 1-105.

Linnavuori R. \& DeLong D.M. 1977a. The leafhoppers (Homoptera: Cicadellidae) known from Chile. Brenesia 12-13: 163-267.

Linnavuori R. \& DeLong D.M. 1977b. The genus Acinopterus (Homoptera: Cicadellidae) in Mexico and the Neotropical Region. Entomological News 88 (9-10): 249-254.

Linnavuori R. \& DeLong D.M. 1978a. The genus Arrugada in Bolivia and Peru. Journal of the Kansas Entomological Society 51 (2): 174-184.

Linnavuori R. \& DeLong D.M. 1978b. Neotropical leafhoppers of the Bahita group (Homoptera: Cicadellidae: Deltocephalinae). A contribution to the taxonomy. Brenesia 14-15: 109-169.

Linnavuori R. \& DeLong D.M. 1978c. Genera and species of Hecalini (Homoptera, Cicadellidae, Deltocephalinae) known to occur in Mexico. Annales Entomologici Fennici 44: 48-53.

Linnavuori R. \& Quartau J.A. 1975. Revision of the Ethiopian Cicadellidae (Hemiptera-Homoptera): Iassinae and Acroponinae. Études du Continent Africain, Fascicule 3: 1-169.

Lu L., Zhang Y. \& Webb M.D. 2011. New tribal placement for the leafhopper genus Evinus Dlabola (Hemiptera: Cicadellidae: Deltocephalinae), with description of a new species from China. Zootaxa 2794: 52-56.

Marino de Remes Lenicov A.M. \& Paradell S. 2009. Argentinean species of the Faltala leafhopper group (Hemiptera: Cicadellidae: Deltocephalinae). Revista de la Sociedad Entomológica Argentina 68 (3-4): 263-269.

Martinson C. 1977. Revision of the genus Osbornellus Ball (Homoptera: Cicadellidae). Ph.D. thesis, The Ohio State University, Columbus, Ohio.

Matsumura S. 1908. Neue Cicadinen aus Europa und Mittelmeergebiet. Journal of the College of Science, Imperial University of Tokyo 23 (6): 1-46.

McKamey S.H. 2000. A review of the New World leafhopper subgenus Texananus (Iowanus Ball) (Hemiptera: Cicadellidae: Deltocephalinae) with a checklist and distribution summary for species in the genus. Proceedings of the Entomological Society of Washington 102 (1): 212-224. 
McKamey S.H. 2001. Review of the Nearctic species of Limotettix (Scleroracus Van Duzee) leafhoppers (Hemiptera: Cicadellidae: Deltocephalinae). Proceedings of the Entomological Society of Washington 103 (3): 686-753.

McKamey S.H. 2003a. Review of the New World leafhopper genus Excultanus Oman (Hemiptera: Cicadellidae: Deltocephalinae). Proceedings of the Entomological Society of Washington 105 (2): 292299.

McKamey S.H. 2003b. Some new generic names in the Cicadellidae (Hemiptera: Deltocephalinae, Selenocephalinae). Proceedings of the Entomological Society of Washington 105 (2): 447-451.

Metcalf Z.P. 1964. General Catalogue of the Homoptera. Fascicle VI. Cicadelloidea. Bibliography of the Cicadelloidea (Homoptera: Auchenorrhyncha). U.S. Department of Agriculture, Agriculture Research Service, Washington D.C.

Meyer-Arndt S. \& Remane R. 1992a. Phylogenie und Speziation der Fieberiellini Wagner, 1951 (Homoptera: Auchenorrhyncha: Cicadellidae). Teil 1. Marburger Entomologische Publikationen 2 (7): $1-387$.

Meyer-Arndt S. \& Remane R. 1992b. Phylogenie und Speziation der Fieberiellini Wagner, 1951 (Homoptera: Auchenorrhyncha: Cicadellidae). Teil 2. Anhang und Tafeln. Marburger Entomologische Publikationen 2 (7): 388-756.

Morrison W.P. 1973. A revision of the Hecalinae (Homoptera: Cicadellidae) of the Oriental region. Pacific Insects 15 (3-4): 379-438.

Nielson M.W. 1968. The Leafhopper Vectors of Phytopathogenic Viruses (Homoptera, Cicadellidae). Taxonomy, Biology, and Virus Transmission. U.S. Department of Agriculture Technical Bulletin No. 1382, Washington D.C.

Nylander J.A.A. 2004. MrModeltest v2. Program distributed by the author. Evolutionary Biology Centre, Uppsala University.

Ohara N. \& Kogure K. 2012. A new species of the genus Parabolopona (Hemiptera, Cicadellidae, Deltocephalinae, Drabescini) from the Central Ryukyus, Japan. Japanese Journal of Systematic Entomology 18 (2): 203-208.

Oman P.W. 1941. A generic revision of the Nearctic Cicadellidae (Homoptera). Ph.D. thesis, George Washington University, Washington D.C.

Oman P.W. 1943. A generic revision of the Nearctic Cicadellidae (Homoptera). [Summary of Doctoral thesis]. The George Washington State University Bulletin 1941-43: 14-17.

Oman P.W. 1949. The Nearctic leafhoppers. A generic classification and check list. Memoirs of the Entomological Society of Washington 3: 1-253.

Oman P.W. 1971. The leafhopper subfamily Koebeliinae. In: Asahima S., Gressitt J.L., Hikada Z., Nishida T. \& Namura K. (eds) Entomological Essays to Commemorate the Retirement of Professor K. Yasumatsu: 129-139. Hokuryukan, Tokyo.

Oman P.W. 1985. A synopsis of the Nearctic Dorycephalinae (Homoptera: Cicadellidae). Journal of the Kansas Entomological Society 58 (2): 314-336.

Oman P.W., Knight W.J. \& Nielson M.W. 1990. Leafhoppers (Cicadellidae): a Bibliography, Generic Check-list, and Index to the World Literature 1956-1985. CAB International Institute of Entomology, Wallingford.

Rakitov R., Villalobos E., Brightsmith D., Dietrich C. \& DeCarlo E. 2005. Mud-puddling (aggregation and feeding at moist ground) in leafhoppers. In: Abstracts of Talks and Posters, 12th International 
Auchenorrhyncha Congress, 6th International Workshop on Leafhoppers and Planthoppers of Economic Significance, August 7-12, 2005: P3, P4. University of California, Berkeley.

Ronquist F., Teslenko M., van der Mark P., Ayres D.L., Darling A., Hohna S., Larget B., Liu L., Suchard M.A. \& Huelsenbeck J.P. 2012. MrBayes 3.2: Efficient Bayesian phylogenetic inference and model choice across a large model space. Systematic Biology 61 (3): 539-542. http://dx.doi.org/10.1093/sysbio/ sys029

Ross H.H. 1968. The evolution and dispersal of the grassland leafhopper genus Exitianus, with keys to the Old World species (Cicadellidae: Hemiptera). Bulletin of the British Museum (Natural History): Entomology 22: 1-30.

Stiller, M. 1986. A new genus, Proekoides (Homoptera: Cicadellidae) described from fynbos in South Africa. Journal of the Entomological Society of Southern Africa 49 (1): 153-158.

Stiller M. 2001. The Afrotropical leafhopper genus Gunghuyana Distant, 1910 with the description of a new genus (Homoptera, Cicadellidae, Deltocephalinae, Athysanini). Deutsche Entomologische Zeitschrift 48 (1): 93-115.

Stiller M. 2009. A revision of the South African leafhopper genus Drakensbergena Linnavuori (Hemiptera: Cicadellidae: Drakensbergeninae). Zootaxa 2132: 1-39.

Stiller M. 2010a. Revision of the Southern African leafhopper genus Pravistylus (Hemiptera, Cicadellidae, Deltocephalinae). Zootaxa 2468: 1-81.

Stiller M. 2010b. Revision of Vilargus Theron (Hemiptera: Cicadellidae: Deltocephalinae) from South Africa. Zootaxa 2674: 1-25.

Swofford D.L. 1998. PAUP*b10. Phylogenetic Analysis Using Parsimony (*and Other Methods). Version 4. Sinauer Associates, Sunderland, Maryland.

Tamura K., Peterson D., Peterson N., Stecher G., Nei M. \& Kumar S.. 2011. MEGA5: Molecular Evolutionary Genetics Analysis using maximum likelihood, evolutionary distance, and maximum parsimony methods. Molecular Biology and Evolution 28 (10): 2731-2739. http://dx.doi.org/10.1093/ molbev/msr121

Theron J.G. 1973. Southern African species of the genus Tetartostylus Wagner (Hemiptera: Cicadellidae). Journal of the Entomological Society of Southern Africa 36 (2): 229-234.

Theron J.G. 1974. The Naudé species of South African Cicadellidae (Hemiptera). III. Species assigned to the genera Chlorotettix Van Duzee, Thamnotettix Zetterstedt, Euscelis Brullé, Scaphoideus Uhler and Selenocephalus Germar. Journal of the Entomological Society of Southern Africa 37 (1): 147-166.

Theron J.G. 1979. Cicadellidae (Hemiptera) associated with the ganna bush, Salsola esterhuyseniae Botsch. Journal of the Entomological Society of Southern Africa 42 (1): 77-88.

Theron J.G. 1980. Notes on some southern African Cicadellidae described by Stål in "Hemiptera Africana". Journal of the Entomological Society of Southern Africa 43 (2): 293-298.

Theron J.G. 1984a. Leafhoppers (Hemiptera: Cicadellidae) associated with the renosterbos, Elytropappus rhinocerotis Less. I. The genus Renosteria Theron. Journal of the Entomological Society of Southern Africa 47 (1): 83-97.

Theron J.G. 1984b. Leafhoppers (Hemiptera: Cicadellidae) associated with the renosterbos, Elytropappus rhinocerotis Less. II. The genera Cerus Theron and Refrolix gen. nov. Journal of the Entomological Society of Southern Africa 47 (2): 217-230. 
Theron J.G. 1987. Southern African species of the genus Hypacostemma Linnavuori (Homoptera: Cicadellidae: Selenocephalinae). Journal of the Entomological Society of Southern Africa 50 (2): 447453.

Vilbaste J. 1965. On the genus Aconura Leth. (Homoptera, Iassidae). Notulae Entomologicae 45: 3-12. Viraktamath C.A. 1998. A revision of the leafhopper tribe Paraboloponini (Hemiptera: Cicadellidae: Selenocephalinae) in the Indian subcontinent. Bulletin of the Natural History Museum (Entomology) 67 (2): 153-207.

Viraktamath C.A. 2004. A revision of the Varta-Stymphalus generic complex of the leafhopper tribe Scaphytopiini (Hemiptera: Cicadellidae) from the Old World. Zootaxa 713: 1-47.

Viraktamath C.A. \& Anantha Murthy H.V. 1999. A revision of the leafhopper tribe Scaphytopiini from India and Nepal. Senckenbergiana Biologica 79 (1): 39-55.

Viraktamath C.A. \& Gnaneswaran R. 2009. Three new species of Goniagnathus (Hemiptera: Cicadellidae) from the Indian subcontinent with description of a new subgenus. Zootaxa 2224: 51-59.

Viraktamath C.A. \& Mohan G.S. 1994. Description of Scaphodhara a new genus related to Scaphoideus (Homoptera: Cicadellidae) and five new species from South India. Entomon 19 (1\&2): 13-22.

Viraktamath C.A. \& Mohan G.S. 2004. A revision of the deltocephaline leafhopper genus Scaphoideus (Hemiptera: Cicadellidae) from the Indian subcontinent. Zootaxa 578: 1-48.

Viraktamath C.A. \& Viraktamath S. 1989. New species of Chloropelix Dist. and Bumizana Lind. from Indian subcontinent (Insecta, Homoptera, Auchenorrhyncha: Cicadellidae). Reichenbachia 27 (1): 1926.

Wagner W. 1951. Beitrag zur Phylogenie und Systematik der Cicadellidae (Jassidae) Nord- und Mitteleuropas. Commentationes Biologicae 12: 1-44.

Webb M.D. 1981. The Asian, Australian and Pacific Paraboloponinae (Homoptera: Cicadellidae). Bulletin of the Natural History Museum (Entomology) 43: 39-76.

Webb M.D. 1999. Identity of Bythoscopus ignicans Walker, 1857 (Hemiptera: Auchenorrhyncha: Cicadomorpha: Cicadellidae: Stegelytrinae). Reichenbachia 33 (14): 111-114.

Webb M.D. \& Godoy C. 1993. Review of the leafhopper tribe Scaphytopiini (Homoptera: Cicadellidae: Deltocephalinae) with a key to genera. Journal of Natural History 27 (2): 423-427. http://dx.doi. org/10.1080/00222939300770181

Webb M.D. \& Heller F.R. 1990. The leafhopper genus Pseupalus in the Old World tropics, with a check-list of the Afrotropical and Oriental Paralimnini (Homoptera: Cicadellidae: Deltocephalinae). Stuttgarter Beiträge zur Naturkunde Serie A (Biologie) 452: 1-10.

Webb M.D. \& Vilbaste J. 1994. Review of the leafhopper genus Balclutha Kirkaldy in the Oriental region (Insecta: Homoptera: Auchenorrhyncha: Cicadellidae). Entomologische Abhandlungen (Dresden) 56 (3): 55-87.

Webb M.D. \& Viraktamath C.A. 2009. Annotated check-list, generic key and new species of Old World Deltocephalini leafhoppers with nomenclatorial changes in the Deltocephalus group and other Deltocephalinae (Hemiptera, Auchenorrhyncha, Cicadellidae). Zootaxa 2163: 1-64.

Wei C., Webb M.D. \& Zhang Y. 2007a. Revision of the Oriental leafhopper genus Toba with description of a related new genus (Hemiptera: Cicadellidae: Stegelytrinae). European Journal of Entomology 104: 285-293. 
Wei C., Webb M.D. \& Zhang Y. 2007b. A remarkable new leafhopper genus and species from Thailand (Hemiptera: Cicadellidae: Stegelytrinae) with modified legs and 'hairy' eggs. Systematic Entomology 32 (3): 539-547. http://dx.doi.org/10.1111/j.1365-3113.2007.00381.x

Wei C., Webb M.D. \& Zhang Y. 2008. The identity of the Oriental leafhopper genera Cyrta Melichar and Placidus Distant (Hemiptera: Cicadellidae: Stegelytrinae), with description of a new genus. Zootaxa 1793: 1-27.

Wei C., Webb M.D. \& Zhang Y. 2010. A review of the morphologically diverse leafhopper subfamily Stegelytrinae (Hemiptera: Cicadellidae) with description of new taxa. Systematic Entomology 35 (1): 19-58. http://dx.doi.org/10.1111/j.1365-3113.2009.00480.x

Wei C., Zhang Y. \& Webb M.D. 2006. New synonymy in the leafhopper genus Stegelytra Mulsant and Rey and description of a new genus (Hemiptera: Cicadellidae: Stegelytrinae). Journal of Natural History 40 (35-37): 2057-2069. http://dx.doi.org/10.1080/00222930601046378

Wei C., Zhang Y. \& Webb M.D. 2008. Minucella, a new leafhopper genus from China (Hemiptera: Cicadellidae: Stegelytrinae). Zootaxa 1854: 33-44.

Weintraub P.G. \& Beanland L. 2006. Insect vectors of phytoplasmas. Annual Review of Entomology 51: 91-111. http://dx.doi.org/10.1146/annurev.ento.51.110104.151039

Wheeler A.G. 1989. Grypotes puncticollis (Homoptera: Cicadellidae), a Palearctic pine-feeding leafhopper new to North America. Proceedings of the Entomologcial Society of Washington 91 (3): 350-354.

Whitcomb R.F. \& Hicks A.L. 1988. Genus Flexamia: new species, phylogeny, and ecology. Great Basin Naturalist Memoirs 12: 224-323.

Yang L. \& Chen X.-S. 2011. Review of bamboo-feeding leafhopper genus Mukaria Distant (Hemiptera: Cicadellidae: Mukariinae) with description of a new species from China. Zootaxa 2882: 27-34.

Zahniser J.N. 2005. A new genus of Deltocephalinae (Hemiptera: Cicadellidae) from Ecuador and a new synonym of Luheria constricta Osborn with illustrations of the female. Annals of the Entomological Society of America 98 (5): 653-657. http://dx.doi.org/10.1603/0013-8746(2005)098[0653:ANGODH] 2.0.CO;2

Zahniser J.N. 2007-present. An Interactive Key to Tribes of Deltocephalinae. Available from: http:// imperialis.inhs.illinois.edu/zahniser/key.asp?key $=$ Delt\&lng $=$ En $\& \mathrm{i}=1 \& \mathrm{keyN}=1$ [accessed March 12, 2013].

Zahniser J.N. 2008a. Seven new species and new distributions of Old World Chiasmini (Hemiptera: Cicadellidae: Deltocephalinae), with a redescription, key to genera, and species checklist for the tribe. Zootaxa 1808: 1-32.

Zahniser J.N. 2008b. Systematics of the leafhopper subfamily Deltocephalinae (Hemiptera: Cicadellidae) and the tribe Chiasmini: phylogeny, classification and biogeography. Ph.D. thesis, University of Illinois at Urbana-Champaign, Urbana, Illinois.

Zahniser J.N. 2010. An enigmatic new leafhopper genus, Protochiasmus (Hemiptera: Cicadellidae: Deltocephalinae), from Brazil. Deutsche Entomologische Zeitschrift 57 (2): 271-274. http://dx.doi. org/10.1002/mmnd.201000024

Zahniser J.N. 2011. On Paraphrodes flavigerus (Evans) with a new synonymy of Paraphrodini with Chiasmini (Hemiptera: Cicadellidae: Deltocephalinae). African Entomology 19 (1): 113-118. http:// dx.doi.org/10.4001/003.019.0119 
Zahniser J.N. \& Dietrich C.H. 2008. Phylogeny of the leafhopper subfamily Deltocephalinae (Insecta: Auchenorrhyncha: Cicadellidae) and related subfamilies based on morphology. Systematics and Biodiversity 6 (1): 1-24. http://dx.doi.org/10.1017/S1477200007002617

Zahniser J.N. \& Dietrich C.H. 2010. Phylogeny of the leafhopper subfamily Deltocephalinae (Hemiptera: Cicadellidae) based on molecular and morphological data with a revised family-group classification. Systematic Entomology 35 (3): 489-511. http://dx.doi.org/10.1111/j.1365-3113.2010.00522.x

Zahniser J.N. \& Hicks A. 2007. Three new species of Icaia Linnavuori (Hemiptera: Cicadellidae: Deltocephalinae) from Peru and distinction of the genus from Athysanella Baker. Zootaxa 1390: 27-39.

Zahniser J.N. \& Nielson M.W. 2012. An extraordinary new genus and three new species of Acostemmini (Hemiptera: Cicadellidae: Deltocephalinae) from Madagascar with comments on the morphology and classification of the tribe. Zootaxa 3209: 28-52.

Zahniser J.N. \& Webb M.D. 2004. Placement of the Faltala Oman leafhopper group (Hemiptera: Cicadellidae: Deltocephalinae) with descriptions of three new species. Annals of the Entomological Society of America 97 (4): 667-674. http://dx.doi.org/10.1603/0013-8746(2004)097[0667:POTFOL]2 $.0 . \mathrm{CO} ; 2$

Zanol K.M.R. 1999. Revisão do gênero Bahita Oman, 1936 (Homoptera, Cicadellidae, Deltocephalinae). Biociências 7 (2): 73-145.

Zhang Y. \& Dai W. 2006. A taxonomic study on the leafhopper genus Scaphoidella Vilbaste (Hemiptera: Cicadellidae: Deltocephalinae) from China. Zoological Science 23 (10): 843-851. http://dx.doi. org/10.2108/zsj.23.843

Zhang Y. \& Webb M.D. 1996. A revised classification of the Asian and Pacific selenocephaline leafhoppers (Homoptera: Cicadellidae). Bulletin of the Natural History Museum (Entomology) 65 (1): $1-103$.

Zimmerman E.C. 1948. Insects of Hawai'i. Volume 4. Homoptera: Auchenorrhyncha. University of Hawai'i Press, Honolulu, HI.

Zwickl D.J. 2006. Genetic algorithm approaches for the phylogenetic analysis of large biological sequence datasets under the maximum likelihood criterion. Ph.D Dissertation, The University of Texas at Austin, Austin, Texas.

Manuscript received: 30 August 2012

Manuscript accepted: 15 March 2013

Published on: 29 May 2013

Topic editor: Koen Martens

Desk editor: Kristiaan Hoedemakers

Printed versions of all papers are also deposited in the libraries of the institutes that are members of the EJT consortium: Muséum National d'Histoire Naturelle, Paris, France; National Botanic Garden of Belgium, Meise, Belgium; Royal Museum for Central Africa, Tervuren, Belgium; Natural History Museum, London, United Kingdom; Royal Belgian Institute of Natural Sciences, Brussels, Belgium; Natural History Museum of Denmark, Copenhagen, Denmark.

Disclaimer: The EJT consortium cannot guarantee that the internal links in this publication will remain operable in the future. 
Appendix 1. List of morphological characters and states.

1. Head width: 0 ) as wide as or wider than pronotum; 1) narrower than pronotum.

2. Crown length: 0 ) parallel margined or somewhat produced; 1) strongly produced.

3. Crown width: 0 ) less than or equal to $2 \mathrm{x}$ width of eye; 1 ) more than $2 \mathrm{x}$ width of eye.

4. Crown surface shape: 0 ) convex; 1) flat or concave.

5. Crown texture at base: 0) shagreen; 1) punctate; 2) irregularly rugose; 3) glabrous and/or with longitudinal or radial striae.

6. Texture of anterior margin of head: 0 ) shagreen; 1) striate or irregularly textured; 2) with numerous carinae; 3 ) with 2 or 3 parallel carinae; 4) with a single transverse carina.

7. Position of ocelli: 0) on crown, posterad of anterior margin; 1) on anterior margin; 2) on face, posteroventrad of anterior margin.

8. Proximity of ocelli to eyes: 0) close; 1) distant.

9. Lateral corner of eye: 0 ) even with or just above lateral carina of pronotum; 1) lying well above lateral carina of pronotum.

10. Mesial margin of eye: 0 ) entire; 1 ) notched.

11. Lateral incision of gena: 0) not incised; 1) somewhat incised; 2) strongly incised, ca $90^{\circ}$ angle.

12. Position of antennal pit: 0 ) near middle or posteroventral (lower) corner of eye; 1 ) near anterodorsal (upper) corner of eye.

13. Antennal ledge: 0 ) absent; 1) weakly carinate to carinate; 2) strongly developed, with ledge.

14. Antennal length: 0 ) short; 1) long; 2) very long.

15. Frontoclypeus length: 0 ) longer than wide; 1 ) shorter than or subequal to width.

16. Frontoclypeus texture: 0 ) shagreen; 1) rugose; 2) punctate; 3) striate; 4) glabrous.

17. Frontoclypeus in profile, anterodorsal part: 0) not inflated; 1) inflated.

18. Frontoclypeus in profile, posteroventral part: 0) not inflated; 1) inflated.

19. Sharply carinate ridge on frontoclypeus below antennal pit: 0) present; 1) absent.

20. Median longitudinal carina on frontoclypeus: 0) absent; 1) present.

21. Long fine setae laterally on frontoclypeus: 0) present; 1) absent.

22. Single fine erect seta on gena near lateral frontal suture: 0 ) present; 1) absent.

23. Proximity of fine erect seta to lateral frontal suture: 0) close; 1) distant.

24. Lateral frontal suture extent: 0 ) reaching ocellus; 1 ) absent or reaching only part way to ocellus.

25. Lateral frontal suture length above antennal pit: 0 ) distinctly shorter than clypeo-genal suture; 1) subequal to or longer than clypeo-genal suture.

26. Lateral frontal suture direction: 0) mesad of ocelli; 1) toward middle of ocelli; 2) laterad of ocelli; 3) strongly divergent, ca. $90^{\circ}$ angle.

27. Ratio of frontoclypeal-loral suture to clypellar-loral suture: 0 ) $1 / 3$ or less; 1) more than $1 / 3$ to $1 ; 2$ ) more than 1.

28. Lorum apex: 0) widely separated from genal margin; 1) near genal margin.

29. Lorum width: 0 ) distinctly narrower than clypellus at base; 1) subequal to or wider than clypellus at base.

30. Clypellar suture completeness: 0) complete; 1) faint or obsolete medially.

31. Clypellar suture: 0) straight; 1) arcuate.

32. Clypellus shape: 0) tapered apically or parallel-sided; 1) expanded apically ovoid.

33. Clypellus apex: 0 ) not far surpassing normal curve of gena; 1 ) far surpassing normal curve of gena; 2) not attaining normal curve of gena.

34. Clypellus in lateral aspect: 0) not inflated; 1) inflated.

35. Apical margin of clypellus: 0 ) notched or concave; 1) straight or convex.

36. Pronotum lateral carina: 0) absent; 1) present.

37. Pronotum transverse striae: 0) absent; 1) present.

38. Forewing veins: 0) not raised, elevated, or carinate; 1) raised, elevated, or carinate. 
39. Number of anteapical cells: 0) 3; 1) 2 .

40. R1 position: 0 ) basad of Rs; 1) distad of Rs.

41. Reflexed costal veins: 0) absent; 1) present.

42. Crossvein m-cu2: 0) absent (inner anteapical cell open); 1) present (inner anteapical cell closed).

43. A1 crossvein between A1 and claval suture: 0) absent; 1) present.

44. A veins: 0 ) gradually curved distally, meeting claval suture obliquely; 1) strongly curved distally, meeting claval suture at ca $90^{\circ}$ angle.

45. A1-A2 crossvein: 0) absent; 1) present.

46. Appendix: 0) absent or reduced; 1) restricted to anal margin; 2) large, extending around wing apex.

47. Hind wing submarginal vein: 0 ) complete; 1 ) obscured apically.

48. Protrochanter: 0) attaining posterior margin of mesosternum; 1) not attaining posterior margin of mesosternum (mesosternum enlarged).

49. Protrochanter stout ventroapical seta: 0 ) absent; 1) present.

50. Profemur row AV: 0 ) reduced or absent; 1) with numerous ( $>3)$ distinct setae; 2 ) with a few widely spaced distinct setae.

51. Profemur row AV setae, length: 0) short; 1) long.

52. Profemur row AV, apex: 0) without setae in apical $1 / 4 ; 1$ ) with several thin seta in apical $1 / 4$.

53. Profemur intercalary row: 0 ) not in rows or scattered; 1) with five or more fine setae in one row; 2) reduced or absent.

54. Profemur extra row of setae above intercalary row: 0) absent; 1) present.

55. Profemur row AM: 0 ) with only AM1 present; 1) with AM1 and one or more additional proximal setae; 2 ) absent.

56. Profemur dorsoapical setae: 0 ) with 2 apical setae; 1 ) with 1 seta or absent.

57. Profemur row PV basal and median setae: 0) reduced or absent; 1) present; 2) with one distinct median seta.

58. Profemur row PV apical seta: 0) reduced or absent; 1) present.

59. Protibia dorsal margins: 0 ) rounded; 1 ) sharply carinate; 2 ) angulate (not carinate).

60. Protibia row AD: 0) without macrosetae; 1) with 1 macroseta; 2) with 2 or more macrosetae.

61. Protibia row PV macrosetae: 0) numerous; 1) 1 to 4; 2) absent.

62. Mesotrochanter stout apical PV seta: 0) absent; 1) present.

63. Mesotrochanter stout apical AV seta: 0) absent; 1) present.

64. Mesotrochanter extra setae: 0 ) absent; 1) present, with numerous extra thick or fine setae.

65. Mesofemur row AV setae: 0 ) reduced or absent; 1) with numerous distinct setae.

66. Mesofemur row AV setae, length: 0) short; 1) long.

67. Mesofemur row AV apical seta: 0) reduced or absent; 1) present.

68. Metafemur penultimate set of macrosetae: 0 ) none; 1) one; 2) two; 3 ) three.

69. Metafemur penultimate pair, proximity: 0 ) widely separated; 1 ) closely set.

70. Metafemur antepenultimate set of macrosetae: 0 ) none; 1) one; 2) two.

71. Metatibia shape: 0 ) arched throughout its length; 1) straight and distinctly bent apically in dorsal view.

72. Metatibia cross-section shape: 0 ) square; 1) rectangular.

73. Metatibia dorsal surface, distinct proximal setae: 0 ) absent; 1) present.

74. Metatibia row PD setae: 0) alternating long and short; 1) macrosetae interspersed with 3-4 smaller setae.

75. Metatibia row PD setae, length: 0) short to moderate; 1) long, nearly as long as or longer than half length of protibia.

76. Metatibia row AD: 0) with macrosetae only; 1) with macrosetae and smaller intercalary setae.

77. Metatibia row AV macrosetae: 0) 6 or fewer; 1) 7 or more.

78. Metatibia row AV macrosetae, extent: 0 ) extending nearly to base; 1) restricted to apical 2/3 or less.

79. Metatibia row AV: 0 ) with macrosetae only; 1) with macrosetae and smaller intercalary setae. 
80. Platellae at apex of metatibia: 0 ) absent; 1) present.

81. Metatarsomere I length: 0) shorter than tarsomeres II \& III combined; 1) equal to or longer than tarsomeres II \& III combined.

82. Metatarsomere I apex: 0) straight; 1) expanded apically.

83. Metatarsomere I dorsoapical pair of setae: 0 ) absent or reduced; 1) present.

84. Metatarsomere I plantar setae: 0 ) all simple, tapered; 1) with one or more platellae.

85. Metatarsomere I plantar setae arrangement: 0 ) in two rows; 1 ) scattered.

86. Metatarsomere I PV apical seta: 0 ) platellate; 1) tapered.

87. Metatarsomere I apical platellae: 0) 3 or less; 1) 4 or more.

88. Metacoxa macrosetae: 0) with several macrosetae along midline; 1) absent or reduced.

89. Female pygofer macrosetae: 0$)$ reduced $(<10)$ or absent; 1$)$ present.

90. Ovipositor: 0 ) not protruding far beyond pygofer; 1) apex protruding far beyond pygofer apex.

91. First valvifers: 0 ) not fused; 1) fused anteriorly.

92. First valvula shape: 0 ) convex; 1) not strongly convex to straight; 2) concave.

93. First valvula dorsal sculpturing pattern: 0 ) strigate, concatenate, or reticulate; 1) granulose, maculose, or imbricate (with overlapping scales); 2) with rectangular scales; 3 ) conchoid.

94. Position of first valvula dorsal sculpturing: 0) reaching dorsal margin; 1) distinctly below dorsal margin.

95. Basal elongation of first valvula dorsal sculpturing pattern: 0) absent; 1) present.

96. First valvula distinctly delimited ventroapical subtriangular sculptured area: 0 ) absent; 1) present.

97. Bases of first valvulae: 0 ) not extended anteriorly; 1 ) extended anteriorly.

98. Second valvula shape: 0) broad, gradually tapered; 1) abruptly broadened medially; 2) slender throughout; 3 ) gradually expanded medially or subapically.

99. Second valvula dorsal tooth on shaft: 0 ) absent; 1) present.

100. Second valvula teeth, shape: 0) large and prominent; 1) not large, regularly or irregularly shaped; 2) absent; 3) obtusely triangular, scalene.

101. Second valvula teeth, extent: 0 ) present on apical $1 / 3$ or more; 1) restricted to apical $1 / 4$.

102. Male pygofer macrosetae: 0 ) absent or reduced ( $\leq$ two rows); 1) present, well differentiated into several rows.

103. Pygofer basolateral membranous cleft: 0) absent; 1) present.

104. Valve shape: 0 ) not triangular; 1) triangular, produced posteromedially.

105. Lateral margin of valve, length: 0 ) short, articulating with pygofer at a point; 1) long, with longer length of articulation with pygofer.

106. Valve condition: 0) free; 1) fused to pygofer; 2) fused to subgenital plates; 3) partially fused to pygofer.

107. Subgenital plate shape: 0) subrectangular; 1) triangular; 2) expanded medially and tapered apically; 3) plates fused; 4) rounded, with lateral lobe; 5) elongate, lobate, rising laterally.

108. Subgenital plate macrosetae: 0 ) absent; 1) scattered or irregularly arranged; 2) uniseriate laterally; 3 ) with two lateral rows and/or with other lateral setae; 4) one or two rows in middle of plate; 5) with one or two rows near median margin.

109. Dorsolateral fold on subgenital plate, articulating with style: 0 ) absent; 1) present.

110. Style shape: 0) linear, median anterior lobe not pronounced; 1) broadly bilobed basally, median anterior lobe pronounced.

111. Style preapical lobe: 0) absent or undeveloped; 1) present.

112. Style apophysis: 0 ) not elongate; 1) elongate.

113. Lateral anterior arms of connective: 0 ) widely divergent (connective $\mathbf{T}$ - or bar-shaped, or platelike); 1) somewhat divergent (connective $\mathbf{Y}$ - or $\mathbf{U}$-shaped); 2) closely appressed anteriorly (= "linear").

114. Connective anteromedial or ventral process: 0) absent; 1) present.

115. Processes of the connective or base of aedeagus: 0 ) absent; 1) present. 
116. Connective articulation with aedeagus: 0) articulated; 1) fused.

117. Base of aedeagus: 0 ) not hinged; 1) hinged.

118. Number of gonopores: 0) one; 1) two.

119. Position of gonopore: 0) ventral; 1) apical; 2) dorsal; 3) dorsolateral.

120. Male segment $X$ long lateroventral processes: 0) absent; 1) present.

121. Male segment X: 0) elongate, $2-3$ longer than wide, sclerotized dorsally; 1) not strongly sclerotized dorsally; 2) sclerotized dorsally, not particularly elongate. 
Appendix 2. Morphological data matrix.

11111111111111111111

111111111122222222223333333333444444444455555555555666666666677777777777888888888899999999990000000000111111111122 1234567890123456789012345678901234567890123456789012345678901234567890123456789012345678901234567890123456789012345678901

A. Krisnella

Abimwa

Acinopterus

Acostemma

Adama

Aflexia

Agrica

Allygidius

Anoplotettix

Antoerostemma

Aphr

Arrugada

Atanus

Athysanus

Athysanus

Attenuipyg

Bahita

Balclutha

Ballana

Bandaromimus

Bhatia

Bonaspeia

Brazosa

Cameroon 142

Caranavia

Caruya

Cerrillus

Cerus

Chiasmus

Chimaerotett

Chinaia

Chinaia

Chlo uni

Chlor_rug

Chlorope

Cicadula

Citorus

Cochlorhinus

Colladonus

Copididonus

Dagama

Dagulus

$101132100010200000100010021110110001100 ? 0 ? ? 0 ? 1001100101001020101101211010001100010100010001000100211111100101100101000101$ 100132201010100000100010121110110011100000101100010010100102 ????10121101000110001010001?101000100011001?04121100101100102 1000311000101000001000100111101100111001001000001110101001020111111211010011100010100010100000100101011000001000001000101 $00113410001010000010001002111011000110010100100011101010110101111012110100011100101000111010001002011100 ? 1310010101100100$ 0001031000101000001000100211101100111000001011001110101001020111101211010001100010100011111000100101111100101110101000001 $00013010 ? 1100000001000001100001000101 ? ? ? ? ? ? ? ? ? ? 00100100000021100101211010001110010100131100010000001011100121110$ ? ???00?02 $00013310011010001010001001010001011110000010 ? 10000 ? 01000000101011012110101011100101001 ? 1100000000001011 ? 04131110100000102$ $000030100110100000101000011110110011100 ? 01101100010010000102 ? 100101211010001100010100010100010000011011100131110100000102$ $10003010011011000010100002111011001110001010010000 ? 01000 ? ? 01 ? 100101211010001100010100010100000100011011100101110100000101$

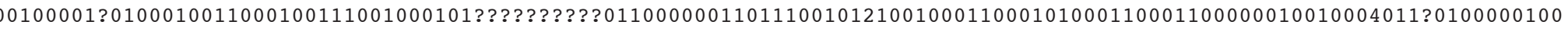
$0011310100102001000001 ? 100101000000111010 a 0000100211000010021101111211010001100010111110001100000001010010510011000000101$ 0 a11211000101001001000010?11101100010100000001000110100011220101111b11000001110010100?110??111010302?01100121110100000100 $00003010 ? 1100000001000000111101100111001001001000100100000012100101211010001110010100100 ? ? ? ? ? ? ? ? ? ? ? ? ? 11100121110100000101$

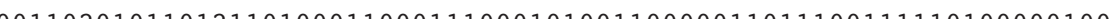
011004 ??00210000001001?10?110010001111????0000?011102001110201011010?01001011110011100?1000111000001001100101110100000101 0001321001100000001000000211100001111001101111000100100001010101101211010001110010100100100000100011011100131110101000101 $100000100010000000100000100110110010001 ? 0000010000 ? 01001000120000 ? 0211010001110010100011100111010302 ? 11100121110100000101$ 0000311000100000001000000211101000111001001001000100100000011100101211010001100010100010101000100011011100121110100100101 0000301001100100001000000211101100111001001001000100100000011100101211010011110010100010100100000001001100121110200100101 $00113110001111000000000002111011001110010010010000 ? 010000001 ? 100111211010001100010100100101000100011011 ? 00101110101000002$ $00013110 ? 0101000001000100211101000111000011010000100100 ? 01021101101211010001100010100010101000100001001100131110100000002$ $101030100000000000100000011110110001100100 ? 0110001001000010111011012110100011100101000 ? 0$ ?????????????11100121110100000101 1001301010101000000100100210110001111001011011000100000001012100101211010001100010100010100000100011011100101110100000101 0000301001100000001000000111101100111001001001000100100000012100101211010001110010100100100000000000011100121110101000101 $000131100010000000100 ? ? 01211101101111001101011000100101001011100101211010001110010100101$ ????????????01100121110100000102 $10112311001010010010000100111011001110010001010000 ? 0100011012101111211010001110010100100100000100000011100101110100000102$ $00003010 ? 110100000100010021110110011100 a 0 a 10 ? 0000100100001011101101211010001100010100010101000100001011100111110100000102$ 0001010100201000001000110111001000100001001002000110200001021101101130000000110100000011010111000103000100101110200010201 $00010011 ? 011010000101001$ ??1011?1001010010010110000?010010001?0001111?00100011000101?100110?000?00012?11100101110100000101 10000021 ?11122000000101011101011201000???????00100?01000010110010?1201010001100010100101010001000001100?10500011010000102 $001000100010000000101000021110100011100100100100010010000102010010121101000110001011001010000001010101100012111010000010 ?$ $000000100110000000100000121110000011100100100100010010000001 ? 1001012110100011100101001 ? 0100000000001011100121110101000001$ $00111410 ? 0210002001101 ? 10 ? 000010001001010000001001102021112111011010 ? 00001000100010000 ? 1010111010002 ? 00 ? ? 110 ? 100100000100$ $00000011 ? 0100100001000000211100100111001000001000010100001011100101211010001110010100011100010000101010100141100100000100$ 001133100010101300100011 ? ?1110010011100000101200111010101121?101111211010001100010100010101000100011001100111110101000102 0001311100100000001000000111001100111001010001000100100011021101101211010001110110110010101000100011011100121110100100100 0000301000100000001000000211101100111001000001000100100001011100101211010001110010100010100000000100011100121110100000201 $000030100110000000100000011110010011100100 a 0 a 1000100100000020100101211010011110010100110101000100011011100121100100000102$ 0001301000101100000000100211101100111000101011000100101011011100101211010011100010100010101000100011011100131110100000112 $00003010 ? 110010000100000010100000011101 ? 001001000100100000011 ? 001012110100 ? 11100101000 ? ? 100010100001011100121110100000101$ 
11111111112222222223333333333344444444455555555556666666667777777777888888888999999999111111111111111111 1234567890123456789012345678901234567890123456789012345678901234567890123456789012345678901234567890123456789012345678901

Deltocephalus DeltocephAus

Doratura

Dorydiella

Drabescus

Drakensbergen

Driotura

Dwightla

Elymana

Eryapus

Euacanthella

Eupelix

Eusaama

Euscelis

Eusceloide

Eutettix

Evinus

Excultanus

Fieberiella

Flexamia

Gcaleka

Gcaleka

Ghanally

Glossocratus

Goniagnathu

Grypotes

Gurawa

Hecalus

Hecullus

Hishimonus

Hishimonus

Hypacostemma

Ikelibeloha

Iturnoria

Jafar

Japananus

Kinonia

Kinrentius

Kinrentius

Koebel

Korana

Kramerana

Laevicephalu

Limotettix

Listrophora

Loipothea

$00003010 ? 110000000100000010101 ? 000101001011001000100100000011100101211010001110010100101100110000001011100121110200100101$ $10010010 ? 11000000010000011001010001010010110110001101000010211011012110100 ? 1100010100110100011010302 ? 11100121110200100101$ $00013110 ? 0101000001000001201101000101$ ???????2000110100001020101101211010001110010100011010111000103000100111110200010201 0011311000100000001000000111101100111001001000000100100011020100101211010001110010100011100010000001011100121100100000102

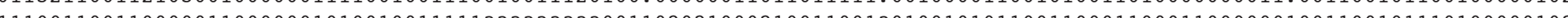
$00003000211000100010100012100010001013233 ? 3232000110100011021101111211010001100010100010010121000103011100101110200010101$

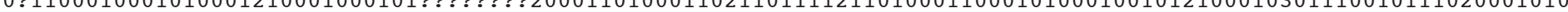
$0010331000111010001010110 ? 1110010011100000111200111010101102 ? 101111211011001100010100010111000100200101100111010101000100$ $000100100110010000100000010101010011100100101100010010000001 ? 100101211010011110010100010100010000001011100121100100000100$

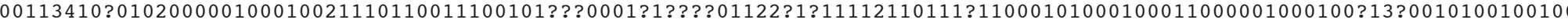
01113101 ? 0211100001001 ? 100000010000010 ????????01210100?110111011111?10000?1100001100111????????????10110550011010000100

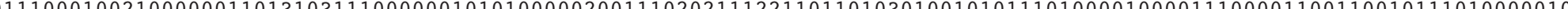
0003010010011000001011100121110100000202

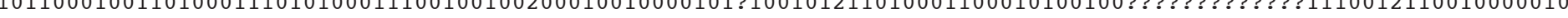

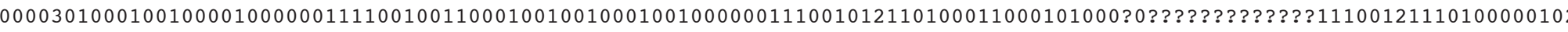
$0100000101200000001000010 ? 011000000100110010010000 ? 01000010121000 ? 021101000111001010001 ? 000111010302 ? 01110401110100000101$ 1001301001101000001000100211101100111001001011000100101001021110101211010001100010100110101000000001011100121110100000102 0001321000101000001000100311101100111001001001001110101001011111101211010001100010100010101000100101011000120000000000111 $00013010 ? 110000000100000 ? 10000000010100 ? 1 ? ? ? ? ? 00010010000001 ? 001101211010001110010100110100010001011011100121110200100102$ (1) $00003010001001001010 \mathrm{c} 000021110010011101 ? 00 ? 0010000 ? 010000101 ? 100101211010011100010100100100000100111011100131110101000101$ $011004100020000000100000011110000011100 ? 0110100011001000110201011012110100011000011000 ? 0100110000301011100121110100000102$ $0000311000101010001000100211101100011000011012001110100001021111111211010001100010100010101000100201011 ? ? 2300100 ? 00100001$ $00000011 ? 01000000010000102111000101110010010010010001000010110000 ? 1211000001110010110011100020000001011100111110100100301$ $0111 d 111 ? 0201000001011 ? 0010010102001110100100000010020210021 ? 0001001 ? 0100001100110100010010111000103001100101110200000101$ $00100110001000000010000000010010001010010000 a 00001001000010101001012110100011100001001 ? 0010111010302 ? 11100121110100000001$ $0110011100100000001000010 ? 1110100011200001101100110010000 ? 0111011012110100011100101001 ? 0000111010002 ? 11100121110101000101$ 0000301000100000001000000011101100111001001011000100100000010100111211010011100010100010100000000001011100131110100001102 $001000110010000000100010113111310001100000 ? 3103003010000101010003121101100$

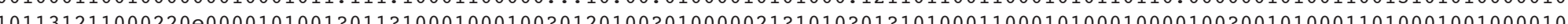
$1000310010002 ? 00001010 ? 0121010110001100101 ? 020001101100 ? ? ? 01 ? 10111121101000110001010001 ? 10 ? 000 ? 00000000 ? 123010 ? 0011000101$ 101131000111201310100010002110110011100101101200111010001111 ? $1 ? 1111311010001100010100100100000 ? 000 ? 1001100121110101000102$ $110101101000000000101010021010010011000 ? 00102100010010000001 ? 101101211010001100010100010100000100011111100101110200001101$ $00010010 ? 11001000010000012010010001010 ? ? ? ? ? ? ? ? 00100100001011101100 \mathrm{f} ? 00100 ? 11100101000 ? 0110111010302 ? 0010014111010001010 ?$ $101134101000000 \mathrm{~g} 00101000120000100111100000 ? 0 ? 201011 ? ? ? 101122 ? 001111212011001100010100120 ? ? ? ? ? ? ? ? ? ? ? ? ? 11100101110100000101$ 01110021001000000010000001111100010100001000000001110200011220000111200000000111110110011010101000301111100111110100000301 1000001000101000001000100111101100011001001011000110101010020100101211011001100010100010100000000001011100121110100000101 $00110 a 1100200000001000011$ ?11101000011?????????011101000010211011012110100011100111001?0000111010002?01100111110100000101 $00013010 ? 110000000100000120100000010100101000100010010000 ? 011100101211010001110010100101100010000001011100121110200000101$ 0000001001100000001000000201001000111001001001000100100001011100101211010001110010100010100110000001011100131110100000101 011?d41110102000001011?00100100100010101000000?0010020210021????1000?000010001010000001?01011100010300110010111020001020 $00003010011000000010100001111001001110011110110000 ? 0100011020101111211010011100010100010100000100101011 ? 02121110100000112$ 
111111111222222222233333333334444444445555555555666666666677777777778888888888999999999900000000001111111111112 1234567890123456789012345678901234567890123456789012345678901234567890123456789012345678901234567890123456789012345678901

Loralia

Luheria

Macrosteles

Magnentius

Menosoma

Mimotettix

Mukaria

Napo

Neoaliturus

Neoheg0 75

Neohegira_br

Neomohunia

Nephotettix

Nesophrosyne

Nesothamnus

Occinirvana

Omanana

Opsius

Orientus

Orosius

Osbornellus Paramesodes Paraphlepsiu Penthimia Penthimidia Penthimiola Peru059 Phlepsius Phlogotettix Placidellus Platymetopius Polyamia Portanus Paradorydiu Paradorya Proceps Protochiasmus Pseudophlep Renonus

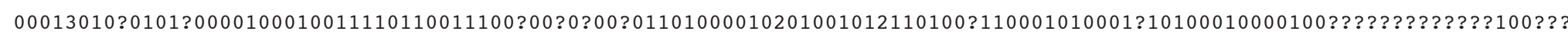
$00013210011010000010001002111011001110000000100011001010010201 ? 1101211010001100010100010101000100001011000111010000000112$ $00000010 ? 0100000001000010 ? 1100000010101 ? 001001000030100000012000100211010001110010100111100110000101011100121110100000101$ $0012112100102002011003213 ? 1010110001111010010120031101000114$ (1)

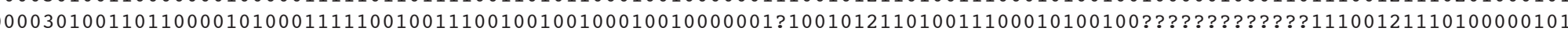
$000132010011200310100001 ? ? 110011011110010000010100101000000121000 ? 0211010011110010100100100100000001001100111110101101101$

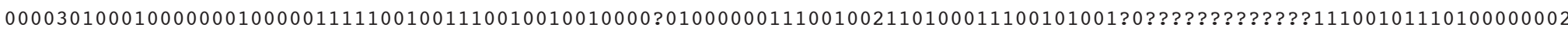
$00003010011000000010000001111011001110010010 a 1000100100000011100101211010001110010100010101010000201011100121110100001101$

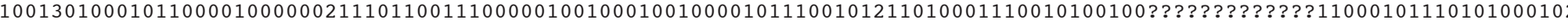
$00013010011010000010000011111011001110000010010001001000010111011012110100011100101000 ? 1101000100013011000101110101000102$ 0001301021100100001010000210101100101002101001000020100000012100020211010011100010100011100000000201011100121110100001101

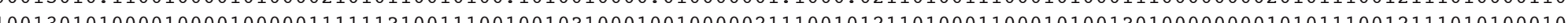
(1) $01103401 ? 011020000100011 ? ? 1111 ? 11010100 ? 1010010000 ? 010000021 ? 00010121100000110001011001110 ? 001 ? 0 ? 0 ? 1011100141110100000101$ $000030100010100000101000121110110011100 ? 1 ? 100100010010000001 ? 100101211010001100010100010100000100011011100101110101000101$ $001030000010001000100000011110110011101 ? 001001000100000000011100101211010001100010110010100000100000011100121110101001101$ $1001401010101 ? 0000100000021110110011100000111100010010100101 ? 100101211010001100010100010101000100011011100101110101000101$ 0001301031100000001010001211101100101001001021000100100000013100100211010011100010100010100010000202311100121110100001101 $000134100010000 \mathrm{e} 00100000011111 ? 0011110011010110 ? 01001000010111011010110100011100101001 ? 0100000000000011 ? 04121110100000112$ $100132101010210000100010011111 ? 100010001000002001110111011010 ? 11101$ ???01101110000010011010000010010301110010100100000010 0000311000101000001000000111101100111001001001000100100001011100101211010001110010100010100000000101011100121110100000100 $\begin{array}{ll}\text { Pachytettix } & 0000311000101000001000000111101100111001001001000100100001011100101211010001110010100010100000001101011100121110100000100 \\ \text { Parabolopona } & 0000031000111100001000010 ? 1110010011100100 ? 1010000 ? 0100000011101111211010011110010100100101100100101011100101110101000101 \\ \text { Paralimnus } & 00013010 ? 1100000001000000100000000101001011001000100100000011100100211010011110010100111101110000001011100121110200000100 \\ \text { Paramesodes } & 00003010 ? 1100 ? 0000100000 ? 20001 ? 0001110000000010 ? 010010000002 ? ? 001012110100 ? 110001 ? ? ? ? ? ? 0 ? ? ? ? ? ? ? ? ? ? ? ? 11100121111200100100\end{array}$ $\begin{array}{ll}\text { Pachytettix } & 0000311000101000001000000111101100111001001001000100100001011100101211010001110010100010100000001101011100121110100000100 \\ \text { Parabolopona } & 0000031000111100001000010 ? 1110010011100100 ? 1010000 ? 0100000011101111211010011110010100100101100100101011100101110101000101 \\ \text { Paralimnus } & 00013010 ? 1100000001000000100000000101001011001000100100000011100100211010011110010100111101110000001011100121110200000100 \\ \text { Paramesodes } & 00003010 ? 1100 ? 0000100000 ? 20001 ? 0001110000000010 ? 010010000002 ? ? 001012110100 ? 110001 ? ? ? ? ? ? 0 ? ? ? ? ? ? ? ? ? ? ? ? 11100121111200100100\end{array}$ $\begin{array}{ll}\text { Pachytettix } & 0000311000101000001000000111101100111001001001000100100001011100101211010001110010100010100000001101011100121110100000100 \\ \text { Parabolopona } & 0000031000111100001000010 ? 1110010011100100 ? 1010000 ? 0100000011101111211010011110010100100101100100101011100101110101000101 \\ \text { Paralimnus } & 00013010 ? 1100000001000000100000000101001011001000100100000011100100211010011110010100111101110000001011100121110200000100 \\ \text { Paramesodes } & 00003010 ? 1100 ? 0000100000 ? 20001 ? 0001110000000010 ? 010010000002 ? ? 001012110100 ? 110001 ? ? ? ? ? ? 0 ? ? ? ? ? ? ? ? ? ? ? ? 11100121111200100100\end{array}$ (1) 000031010010200410100??0?21111?20111100101102201011?0??01110?00111121101010110001010001011010000000101?000131110100000102 00031110011200010100001 ? ? $111011001110010110110000 ? 010000021 ? 101101211010001100010100100100000100101011100121110100000101$

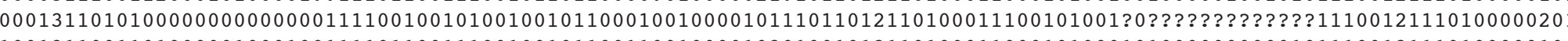
1001311001101000001000100111101100111001001011001100100001020100101211010001100010100010100000000001011100121110100000101 0000301001101100001000000211100100111001001001000100100001011100101211010011100010100100100000100011011100121110101000101 100134101000020400100010121110010010100100101201 ?1111110110?2??11112120110?11000101001?01100001001010??????????????????? $000130100010000000100000011110110011100 ? 0110 ? 1000100100001021100101211010001110010100011100000000000001100121110100000101$ $00003010 ? 1100000010000011000130001010010110110001001000000211011012111010031110010100100100010110001011100121110200100101$ 1010 010100100010010000101000011111 ? 10011100100100100110010000001 ?1001012110100011000101000101001101000010???????????????????

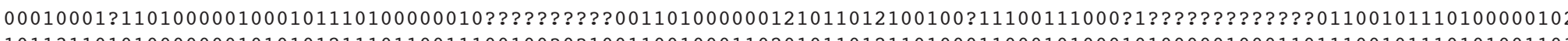
$101131101010000000101010121110110011100100 ? 0 ? 1001100100011020101101211010001100010100010100000100011011100101110101001101$ $00013010 ? 01001000010100011111011001010011010210000 ? 010000001 ? 001101211010001100010100100100010000001011100101110100000101$ $0001301000100 ? 00001000100211101000111000001001001100100001011101101211010001100010100010100000 ? 00001011100131110101000101$ 
11111111111111111111 1234567890123456789012345678901234567890123456789012345678901234567890123456789012345678901234567890123456789012345678901

Scaph0 40

Scaph063

Scaph120

Scaphoidophs

Scaphoidoph

Scaphotettix

Scaphytopiu

Scpy 1

Selenocephal
Stenometopi

Stirellus

Stymphalulus

Taperinha

Tenucephalus

Thailand112

Thamnotettix

Trop_89a

Tropican131

Twiningia

Xestocephalus

Yungasia

Zambial18

Zambial190s

Zambial21

Zambia1220ps

$00013010011101000010000001111001001100011011010000 ? 0100000011100101211010011100010100110101000100011011100121110101000101$ 0013010011101001010000000111001001100011011 ?10000?010000001?1001012110100111000101001101010000000110??????????????????? $110130101110010000101000011110010011100 ? 1011 ? 10000 ? 010000001 ? 100101211010011100010100 a 10100000100011011100121111201000101$ $110130100110010000101000011110110011101 ? 10100100010010000001 ? 100101211010011100010100010100000001001011100121110100000101$ $00013110 ? 1110 ? 00101000010 ? 1101 ? 00011100 ? 00 ? ? ? 10000 ? 01000000120000 ? 02110100 ? 11100101001 ? 110110000000101110012111010000000 ?$ $110131101000000000101000101110010011100 ? 1 ? 10 ? 100010010000001$ ? $100101211010001100010100120 ? ? ? ? ? ? ? ? ? ? ? ? 11100121110101000102$ $1001311000000000010000001110010011100 ? 111111000100100000010100101211010011100010100100101000000100011100121110101000101$

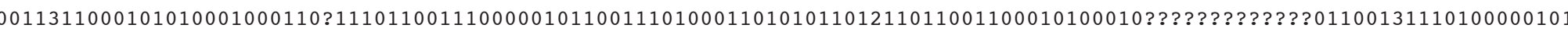
0001301001100000001000000211101100111001001001000100100000021100111211010001110010100011100110000101000100441110100000100 $00000010 ? 1100000001000001101001000001000001001000100100001011101101211010001110010100011010111010302 ? 00100441110100000101$ $0101311010111100001100010 ? 1010010001100000000100010010000 ? 2111001012110110011000101001 ? 01000000000000 ? 110011 ? 1 ? 010 ? 1001 ? ?$

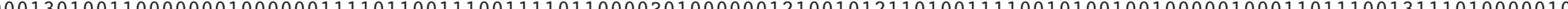
$00003110011001000010000100010010001110010010010000 ? 010000001100010121101001111001010010111112101$ $00000010 ? 01000000010000011110000001000000010010000 ? 0200000011000101211010001110010100110100111000001011 ? 00121110201000101$ $00013411001010140110 ? 001$ ?? $1000100111110100 ? 0 ? 100001011 ? 00001 ? 101011211010001100010100100100000000011011100111110100000102$ $00003010001000000010000002110010001110010010010001001000 ? ? 021101101211010001100010100010100000000011011100121100100000102$ $000030100110000000100000111110110011100100 ? 0 ? 10001001000000111001002110100111100101001 ? 0100011000103011100121110100000101$ $00003010011000000010100001111011001110010010 ? 100010010000001 ? 100100211010001100010100100100000000103011100121110100000101$

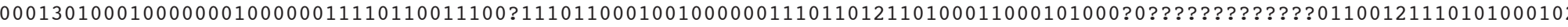

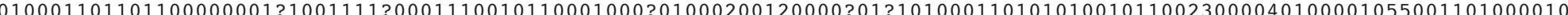
(1) 00013010011001000010100001111011001110011111011000100100000012100101211010011100010100011100000100103011100101110100000100 $00130100110010000101000011110110011100111101100010010000001 ? 100101211010011100010100011100000100103011100101110100000100$ $000030100110010000101000021110110011100110100100010010000001 ? 100101211010011100010100100100000001011011100121110101000102$ 0000001001100000001010000111101100111000111011000100100001012000101211010001100010100010100000000001011100121110100000102

$(0,1)=\mathrm{a} ; \quad(1,2)=\mathrm{b} ; \quad(1,4)=\mathrm{c} ; \quad(0,2)=\mathrm{d} ; \quad(0,3)=\mathrm{e} ; \quad(0,1,2)=\mathrm{f} ; \quad(0,4)=\mathrm{g}$ 